\title{
White-Light Mass Determination and Geometrical Modelling of Coronal Mass Ejections
}

\author{
Dissertation
}

zur Erlangung des mathematisch-naturwissenschaftlichen Doktorgrades

"Doctor rerum naturalium"

der Georg-August-Universität zu Göttingen

im Promotionsprogramm ProPhys

der Georg-August University School of Science (GAUSS)

vorgelegt von

Adam Martin Pluta

aus

Eschwege

Göttingen, September 2018 



\section{Betreuungsausschuss:}

\section{Prof. Dr. Ansgar Reiners}

Sonnenphysik und Stellare Astrophysik, Institut für Astrophysik, Georg-August-Universität Göttingen, Germany

\section{Dr. Volker Bothmer}

Sonnenphysik und Stellare Astrophysik, Institut für Astrophysik, Georg-August-Universität Göttingen, Germany

\section{Mitglieder der Prüfungskommission:}

\section{Referent: Prof. Dr. Ansgar Reiners}

Sonnenphysik und Stellare Astrophysik, Institut für Astrophysik, Georg-August-Universität Göttingen, Germany

\section{Korreferent: Dr. Volker Bothmer}

Sonnenphysik und Stellare Astrophysik, Institut für Astrophysik, Georg-August-Universität Göttingen, Germany

\section{Weitere Mitglieder der Prüfungskommission:}

\section{Prof. Dr. Stefan Dreizler}

Sonnenphysik und Stellare Astrophysik, Institut für Astrophysik, Georg-August-Universität Göttingen, Germany

\section{Prof. Dr. Wolfram Kollatschny}

Extragalaktische Astrophysik und Kosmologie, Institut für Astrophysik, Georg-August-Universität Göttingen, Germany

\section{Prof. Dr. Jens Niemeyer}

Extragalaktische Astrophysik und Kosmologie, Institut für Astrophysik, Georg-August-Universität Göttingen, Germany

\section{Prof. Dr. Ulrich Parlitz}

Biomedical Physics Group, Max Planck Institute für Dynamik und Selbstorganisation, Göttingen, Germany 



\section{Abstract}

Coronal Mass Ejections (CMEs) are explosive large-scale outbursts of the Sun's coronal plasma and magnetic field. They can induce strong geomagnetic storms at Earth, which pose serious threats to space systems, communications and navigation. Hence, arrival predictions of CMEs are of special interest to the humane society. Such predictions require a meticulous analysis of CME properties in the earliest possible stage. Coronagraph observations can provide important insights into the CME kinematics, morphology and mass at CME distances of only a few solar radii away from the Sun. However, the 3-dimensional structure of CMEs can only by analysed, based on their 2-dimensional projection in coronagraph images, which means that they are affected by projection effects.

This thesis has the goal to present the state-of-the-art methods of CME parameterisation derived from coronagraph observations and to discuss arising issues resulting from projection effects. A focus is laid on the measurements of the CME mass and morphology as well as the question under which conditions they can be determined with highest accuracy. Further, the solar mass loss caused by CMEs is investigated. Also, CME mass determination is currently not feasible in real-time and therefore not applicable in actual terrestrial CME arrival predictions. Thus, it is discussed how the CME mass and the CME morphology can be empirically estimated from the CME speed.

The thesis presents a new combined method which enables the measurement of relevant CME kinematics, morphology and mass in a consistent and comparable manner. The two vantage points of the COR2 coronagraphs onboard of the twin NASA STEREO spacecraft are used to apply the method to a set of 122 events with intense brightness. The modelling results are analysed to derive empirical correlations with the CME speed. Further, a CME propagation model - the Drag-Based Model (DBM) - is combined with the GCS model to predict the CME arrival of a sample event at Earth.

It is shown that the largest CME parameterisation uncertainties arise for events emerging from close to the disk centre towards or away from the observer. For these events the term "disk events" is adopted. If an event is seen as disk event in both coronagraphs, the CME morphology can be overestimated by up to a factor of two from stereoscopical modelling. Equally the CME mass of disk events can be overestimated by a factor of 10 and more in the case of overlapping coronal streamers. Therefore, stereoscopical measurements of disk events are not always reliable, at least under a very active background corona. Though, the CME mass $M$ can be estimated from the initial apex velocity $v_{\text {apex }}$ with the empirically derived equation

$$
\log _{10}(M)=3.4 \times 10^{-4} v_{\text {apex }}+15.479 .
$$

This result is used to predict the terrestrial CME arrival of a CME with an Earth-directed initial speed of $1172 \mathrm{~km} / \mathrm{s}$ with the GCS plus DBM model. The CME arrival time and the arrival speed are both strongly affected by the solar wind density and CME mass. For the presented case the arrival prediction limits spread to $\Delta T=59 \mathrm{~h}$ and $\Delta v=748 \mathrm{~km} / \mathrm{s}$ for typical CME mass and solar wind values. It is demonstrated that the derived empirical equation can be very valuable to improve the arrival prediction accuracy. 


\section{Contents}

1 Introduction 3

1.1 Motivation and Objectives of this Work . . . . . . . . . . . . 7

1.2 Thesis Guideline . . . . . . . . . . . . . . . . . . . . . . 9

2 The Sun $\quad 11$

2.1 The Solar Interior . . . . . . . . . . . . . . . . . . . . . . . . 12

2.2 The Solar Atmosphere . . . . . . . . . . . . . . . . . . . . . . . . 14

2.3 The Physics of Coronal Plasma . . . . . . . . . . . . . . . . . . . . 21

2.4 Coronal Large-Scale Structures . . . . . . . . . . . . . . . . . . 26

2.5 The Magnetic Sun . . . . . . . . . . . . . . . . . . . 27

3 Interplanetary Space 33

3.1 Solar Wind . . . . . . . . . . . . . . . . . . . . . 33

3.2 The Interplanetary Magnetic Field . . . . . . . . . . . . . . . . . . . . 34

3.3 The Magnetosphere . . . . . . . . . . . . . . . . . . . . . . 36

3.4 The Heliosphere . . . . . . . . . . . . . . . . . . . . . . . . . 37

4 Coronal Mass Ejections and Associated Phenomena 39

4.1 Flux Rope Morphology . . . . . . . . . . . . . . . . . . . . . . . . . . 41

4.2 Flux Rope Eruption . . . . . . . . . . . . . . . . . . . . . 45

4.3 Solar Flares . . . . . . . . . . . . . . . . . . . . . . . . . . . . . . . . . . . 49

4.4 Post Eruptive Arcades . . . . . . . . . . . . . . . . . . . . . . . 51

4.5 Prominences . . . . . . . . . . . . . . . . . . . . . 51

4.6 Coronal Shock Waves. . . . . . . . . . . . . . . . . . . . 52

5 Observation \& Instrumentation $\mathbf{5 5}$

5.1 Coronagraphs . . . . . . . . . . . . . . . . . 55

5.2 Solar and Heliospheric Observatory $(\mathrm{SOHO}) \ldots \ldots \ldots \ldots 5$

5.3 Solar Terrestrial Relations Observatory (STEREO) . . . . . . . . . . . . . . 57

5.4 Advanced Composition Explorer (ACE) . . . . . . . . . . . . . 63

5.5 Coordinate Systems and Transformations . . . . . . . . . . . . . 64

6 Thomson Scattering $\quad 67$

6.1 Thomson Scattering in the Corona . . . . . . . . . . . . . . . . 67

6.2 Projection Effects . . . . . . . . . . . . . . . . . . . . . . 70

7 Geometrical CME Modelling $\quad 73$

7.1 Graduated Cylindrical Shell Model . . . . . . . . . . . . . . . . . . . 74

7.2 GCS Modelling Results . . . . . . . . . . . . . . . . . . . . 75

8 Combining GCS Modelling and CME Mass Determination 87

8.1 CME Mass Determination . . . . . . . . . . . . . . . . . . 87

8.2 Projection Effects and CME Parameter Study . . . . . . . . . . . . . . . 88

8.3 Mass Overestimation from Coronal Streamers . . . . . . . . . . . . . . . . 101 
8.4 Mass Loss of the Sun . . . . . . . . . . . . . . . . . . . . . 105

9 Application in CME Forecasts $\quad 107$

9.1 Drag Based Model . . . . . . . . . . . . . . . . . . . . . 107

9.2 The DBM plus GCS Model . . . . . . . . . . . . . . . . . . . . . . 110

9.3 Prediction Example . . . . . . . . . . . . . . . . . . . . . 111

9.4 In-Situ Arrival . . . . . . . . . . . . . . . . . . . . . . . . . 114

9.5 Conclusion . . . . . . . . . . . . . . . . . . . 116

10 Summary 117

10.1 Conclusion . . . . . . . . . . . . . . . . . . . . 117

10.2 Outlook . . . . . . . . . . . . . . . . . . . . . . 119

$\begin{array}{ll}\text { Bibliography } & 122\end{array}$ 


\section{List of Abbreviations}

ACE Advanced Composition Explorer

AR Active Region

C3 $\quad \mathrm{SOHO} / \mathrm{LASCO}$ Coronagraph 3

CIR Co-rotating Interaction Region

CME Coronal Mass Ejection

COR2 STEREO/SECCHI Coronagraph 2

DBM Drag-Based Model

DQCS Dipole plus Quadrupole plus Current Sheet

ESA European Space Agency

FITS Flexible Image Transport System

FOV Field-Of-View

FR Flux Rope

FRi3D Flux Rope in 3D

GCS Graduated Cylindrical Shell

HAO High Altitude Obervatory

HCS Heliospheric Current Sheetheet

HEE Heliocentric Earth Ecliptic

HEEQ Heliocentric Earth Equatorial

HI Heliospheric Imager

ICC Ice-Cream Cone

ICME Interplanetary Coronal Mass Eruption

IMF Interplanetary Magnetic Field

KINCAT Kinematic Catalogue

LASCO Large Angle and Spectrometric Coronagraph

LOS Line-Of-Sight

MC Magnetic Cloud 
MHD Magnetohydrodynamics

MSB Mean Solar Brightness

MSS Minimal STEREO separation

NASA National Aeronautics and Space Administration

NCAR Nation Center for Atmospheric Research

NOAA National Oceanic and Atmospheric Administration

PA Position Angle

POS Plane-Of-Sky

ROI Region of Interest

SDO Solar Dynamics Observatory

SECCHI Sun Earth Connection Coronal and Heliospheric Investigation

SEP Solar Energetic Particles

SOHO Solar and Heliospheric Observatory

SR Source Region

SSC Storm Sudden Commencement

STEREO Solar Terrestrial Relations Observatory

SWEPAM Solar Wind Electron Proton Alpha Monitor

TRACE Transition Region and Coronal Explorer

TS Thomson Sphere

USAF United States Air Force 

"Modern society is becoming ever increasingly dependent on space technology for daily routine functions, such as communication, ship and satellite navigation, data transmission, global surveillance of resources, and atmospheric weather. However, if a Carrington-type storm (or a greater intensity one) were to occur now, it could cause much more damage to society than in 1859 when the telegraph (Boteler, 2006) was the latest technology of the time. Therefore, it is crucial to have knowledge about the occurrence of extreme events, and their causes in order to assess their possible impacts on society"

-Lakhina \& Tsurutani (2016) 



\section{Introduction}

The Sun is of essential importance to the life and well being of humanity. Before the discovery of fire, working life was mostly restricted to the time between sunrise and dawn. With the beginning of agriculture the lack of sunshine resulted in fewer harvests and even worse - bad wine. It is thus hardly surprising that we can find proof for the adoration of the Sun in almost every ancient culture. The Egyptians prayed to their sun-god Ra, the German deity was called Sunna or Sol and the Hindus still worship Vishnu. With such a huge cultural influence, it seems likely that the Sun also have raised the astronomical interest of people. The oldest surviving testimonies, the Nebra Sky Disk (1600 BC; Figure 1.1), was an astronomical instrument which had the purpose to predict the solstice. Another example is a Nordic Bronze Age artefact discovered in Denmark, the Trundholm Sun Chariot (1400 BC; Figure 1.2), which might have had the function of a calendar. The Greek philosopher Xenophanes $(\sim 570-475 \mathrm{BC})$ was the first to describe the Sun as natural object which could be interpreted as the birth of astrophysics in European history. Around $300 \mathrm{BC}$ another Greek, the astronomer and mathematician Aristarchus of Samos, leader of the school of Aristoteles, postulated the heliocentric system with the Sun in the centre of the universe (Heath \& Aristarchus of Samos, 1913), which has been later proven by Nicolaus Copernicus (1473-1543 AD). Historically, many insights in the nature of the Sun were gained from observations of sunspots. For instance, around 1611, David and Johannes Fabricius were among the first to study the movement of sunspots with the help of telescopes and concluded that the Sun is rotating around its own axis. Two essential discoveries have to be highlighted in the context of this thesis.

- In 1806 Alexander von Humboldt found that the variation of a compass needle was accompanied by the appearance of auroral lights on the following night and described them with the German term "magnetische Ungewitter" (magnetic storms).

- In 1859 Richard Carrington observed an intense and rapidly occurring brightening in the middle of a sunspot group, which will later be interpreted as a solar flare.

At first, both discoveries seemed pretty unrelated, but soon after the discovery of the magnetic nature of sunspots (Hale, 1908a) it became clear that the solar activity is somehow connected to the geomagnetic activity (Lindemann, 1919). It was later postulated by Chapman \& Ferraro $(1931,1932)$ that transient ejections of solar plasma are the source of this connection. In the modern astrophysical sense, we now understand the Sun is going through an 11-year cycle of activity, that has an extreme physical complexity. The research of the complex solar phenomena has improved our understanding in many areas of physics, including particle physics, plasma physics, spectroscopy and magnetohydrodynamics. But it has also revealed that the Sun can pose a serious threat to Earth and humans.

Such impacts of interplanetary as well as cosmic phenomena at Earth are investigated within a special branch of space physics called "Space Weather" (Bothmer \& Daglis, 2007). Today we know that Earth's magnetosphere provides us with a natural shielding against cosmic matter and space radiation. However, some solar transient phenomena, sometimes summed up under the term "Solar Storm", have shown to overcome this barrier. They can 


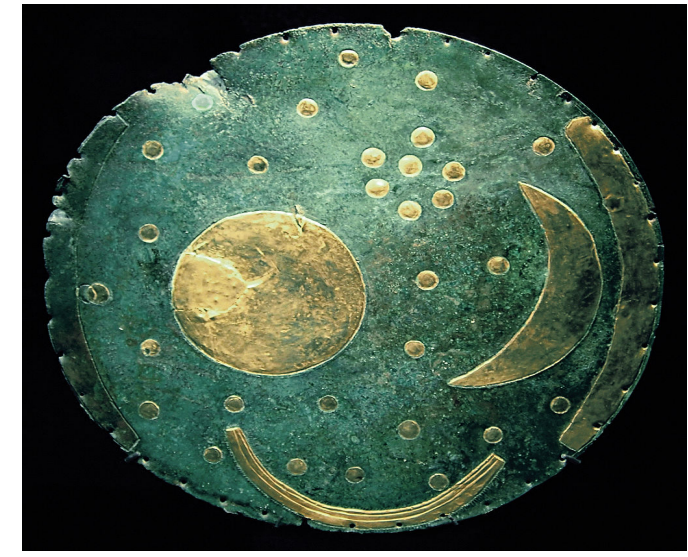

Fig. 1.1: Nebra Sky Disk - Pergamon Museum, Berlin. Credit: D.Bachmann

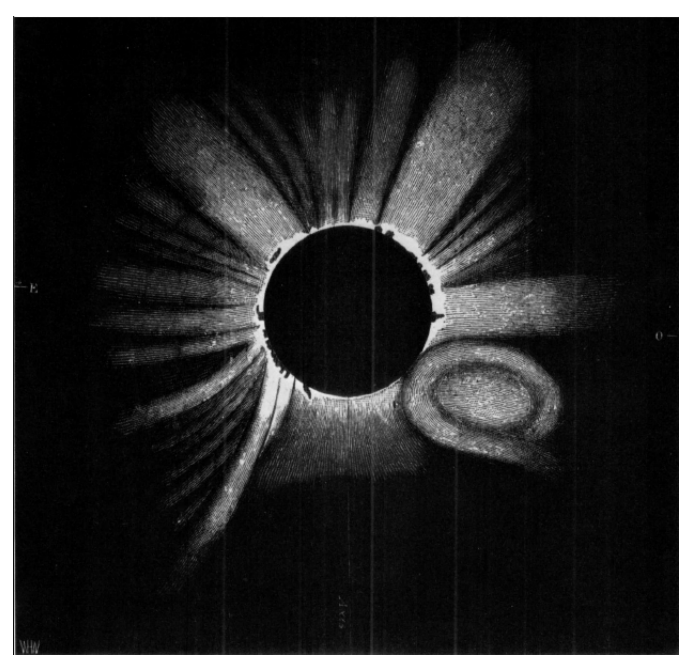

Fig. 1.3: Drawing of a corona during a total solar eclipse on 18th July 1860 by G.Temple at Torreblanca Spain. It is theorised that the bottom right structure might represent a Coronal Mass Ejection (Eddy, 1974).

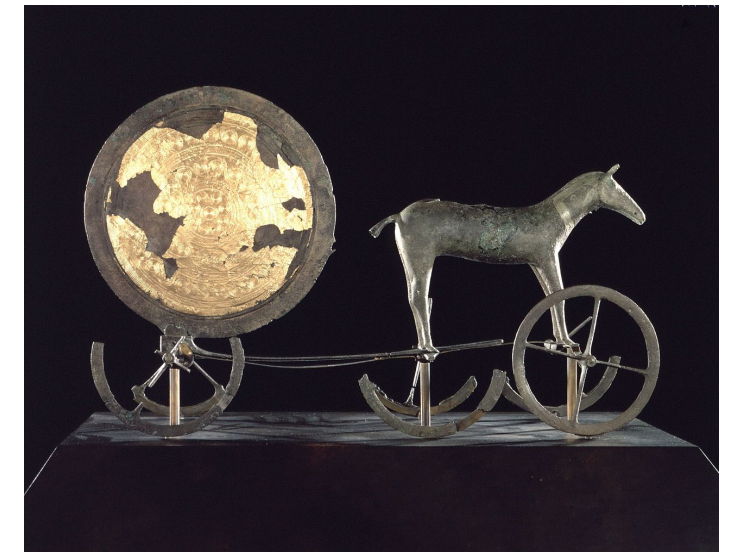

Fig. 1.2: Trundholm Sun Chariot - Nationalmuseet, Denmark. Credit: John Lee

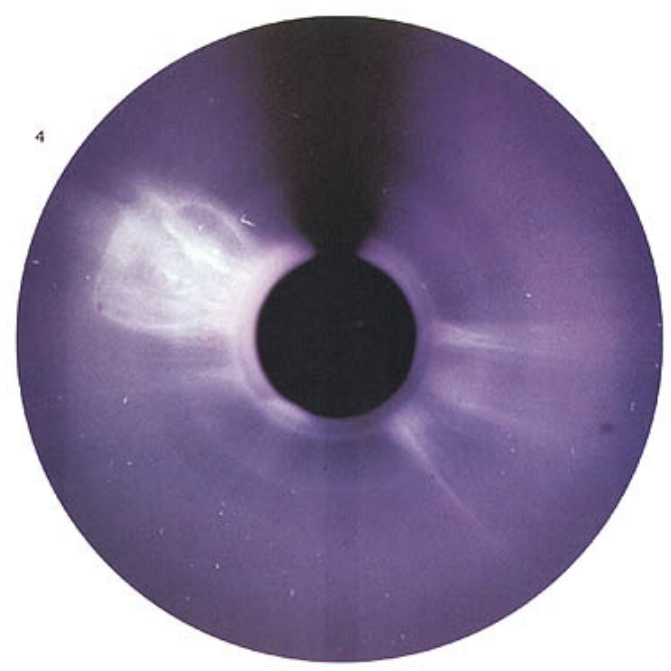

Fig. 1.4: Detection of a CME on December 14, 1971 with the 7th Orbiting Solar Observatory(OSO-7). (NASA) 
induce huge magnetic perturbations in Earth's magnetosphere, the so called "geomagnetic storms". The most important manifestation of solar storms are Coronal Mass Ejections. CMEs are characterised as massive eruptive releases of plasma and magnetic fields from the solar corona. The coronal material of CMEs forms huge blobs which propagate into the heliosphere. They can reach Earth at a distance of 150 million $\mathrm{km}$ in less than a day, due to their enormous speeds of up to 10 million kilometres per hour. Historical observations of CMEs are not documented as the solar corona is only visible to the naked eye during a solar eclipse. Solar eclipses, with a typical duration of about 7 minutes, do not last enough to observe the transient dynamics of CMEs. Though, there are indicators of historical observations of coronal patterns in the solar corona (see Figure 1.3). The first documented discovery of a CME (see Figure 1.4) was made in the Space Age by the coronagraph onboard the seventh Orbiting Solar Observatory (OSO-7) satellite (Tousey, 1973) and the Apollo Telescope Mount (ATM) Coronagraph onboard Skylab (MacQueen et al., 1974).

Within two decades it was realised that CMEs are the most important players in the solar terrestrial relationship and the root of many observed space weather effects on Earth (Gopalswamy, 2016). Until then, solar flares, sudden flashes of increased solar brightness, discovered around 100 years earlier independently by R. Carrington and R. Hodgson (Carrington, 1859a), were considered for this role. CMEs are considered to be the cause of the strongest space weather related effects, posing a serious threat to technical systems, communication and navigation (Cherry, 2002; Thomson et al., 2011; Pulkkinen et al., 2005). These effects often manifest in geomagnetic storms and it is known that the strongest ones are initiated through CMEs (Gosling et al., 1991; Tsurutani et al., 1988; Burlaga et al., 1987) with high velocities and a strong negative magnetic field component (Tsurutani et al., 1995). To this date the Carrington event is the most energetic solar storm, that had yet been observed, and also caused one of the most strongest geomagnetic storms. Widely spread sightings of aurorae at very low latitudes $\left(18^{\circ}\right)$ and outages of telegraphs all over Europe and North America (Baker, 2009) were the results. But there are also other geoeffective phenomena which are closely related to CMEs, for instance Solar Energetic Particles (SEPs), sometimes also termed "Solar Protons Events" or "Proton Storms". They are formed during the eruption phase of intense CMEs or solar flares (Droege \& Schlickeiser, 1986) by an interplanetary shock wave and become accelerated to near relativistic speeds. The fastest particles arrive at Earth within tens of minutes where lower-energy particles take up to one day. Once arrived at Earth's magnetosphere, the magnetic field lines direct them towards the polar regions. Here, the protons can penetrate the magnetosphere and ionise the lower ionosphere (Richard et al., 2002). An overview about the flow of solar mass, momentum and energy from the Sun to Earth is presented in Fig. (1.5).

On May 25, 1967 one of the strongest geomagnetic storms of the 20th century caused disturbances of the American RCA 474L Ballistic Missile Early Warning System in Canada, Greenland and England which nearly resulted in a nuclear war with Russia (Knipp et al., 2016). Besides these social impacts, the risk for technical systems became more evident with the geomagnetic storm on March 13, 1989. This event occurred in the aftermath of a huge CME which was triggered about three and a half days earlier. The accompanied aurora was exceptionally intense and even observable in Southern Europe. The compression of the Earth's magnetic field induced electric currents into the national electricity grid of Canada. As a consequence the province Quebec and their six million inhabitants were cutout from the electrical power supply for nine hours. This furthermore caused a cascading 


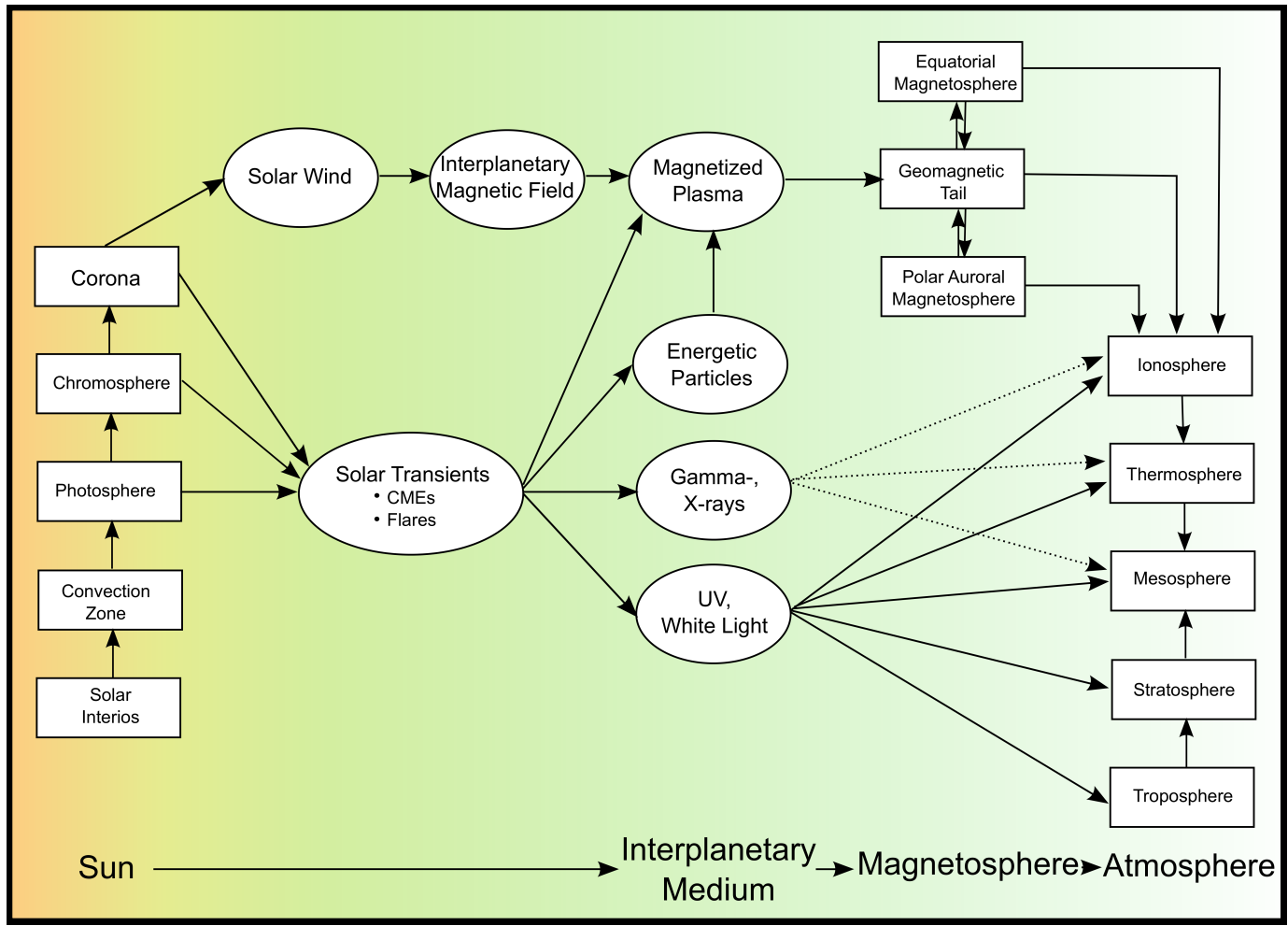

Fig. 1.5: Overview of the flow of solar mass, momentum and energy from the Sun's interior to the different layers of Earth's atmosphere.

collapse of neighbouring power grids (Boteler et al., 1998). Boat and aeroplane navigation systems were distorted by compass errors of ten degrees. Also, turbulences in the ionosphere caused the disturbance of short-wave communication all over the globe. The total economic loss was estimated at 13.2 billion Canadian Dollar (Bolduc, 2002). Other CMEs even disabled communication satellites such as the Canadian Aniks E1 and E2 and the international Intelsat K on 20 January 1994 (Baker et al., 1994), as well as the AT\&T Telstar 401 on January 7, 1997 (Reeves et al., 1998). The increased radiation caused by solar storm also posses serious risk to the health of astronauts and high-altitude flight passengers, particularly when travelling over the poles (Baker et al., 2004). On Halloween 2003 a geomagnetic "super" storm knocked down a part of the high voltage power grid in southern Sweden for up to 50 minutes. It further enforced alternate airline routes due to the increased particle radiation and led to the loss of the Japanese (US\$ 640 million) environment satellite ADEOS-II (Pulkkinen et al., 2005). The economic consequences of Space Weather related effects are enormous. A report by the US National Research Council (Committee On The Societal \& Economic Impacts Of Severe Space Weather Events, 2008) estimated the today's maximum potential economic loss with up to 2 trillion US dollar. Thus, the monitoring of heliospheric propagation and forecasting of the Earth-arrival of solar storms and in particular CMEs is of great interest to our society, in order to take precautions. In 2014, NASA reported that the solar event on July 23, 2012 was at least in the order of the Carrington-event but has missed Earth (Baker et al., 2013). There are also examples for SEP induced space weather effects. Two days after the 1961 September 28 flare, Pioneer 12 measured a large increase in the proton intensity of $2-15 \mathrm{MeV}$ (Bryant et al., 1962). This event was first interpreted as solar protons, encapsulated in the plasma cloud of the correspondent magnetic storm, but later on Rao et al. (1967) concluded from the observation 
of similar events that the protons must have been accelerated by interplanetary shocks. On 28 October 2003 the accelerated protons of the Halloween event led to a temporary failure of the SOHO satellite and the damaging of the ACE satellite (Dmitriev et al., 2004).

On the other side, some solar storm related effects manifest as spectacular light phenomena in the upper atmosphere, namely Auroras (Fig 1.6). The name "Aurora" was coined by Galileo in 1619, after the Roman godness of dawn. They are also known as "Aurora Borealis" or "Northern Lights" in the northern hemisphere and as "Aurora Australis" in the southern hemisphere. Auroras are initiated when the magnetosphere is significantly disturbed by the solar wind. Under certain magnetic configurations, the solar wind initiates magnetic reconnection in the tail of Earth's magnetosphere. As a result two powerful streams of highly-energetic plasma are launched - one towards Earth and one in the opposite direction. The Earth-directed stream can infiltrate plasma particles into the ionosphere at heights above $100 \mathrm{~km}$. Electrons of $\mathrm{keV}$ energies then excite atoms to radiate at specific

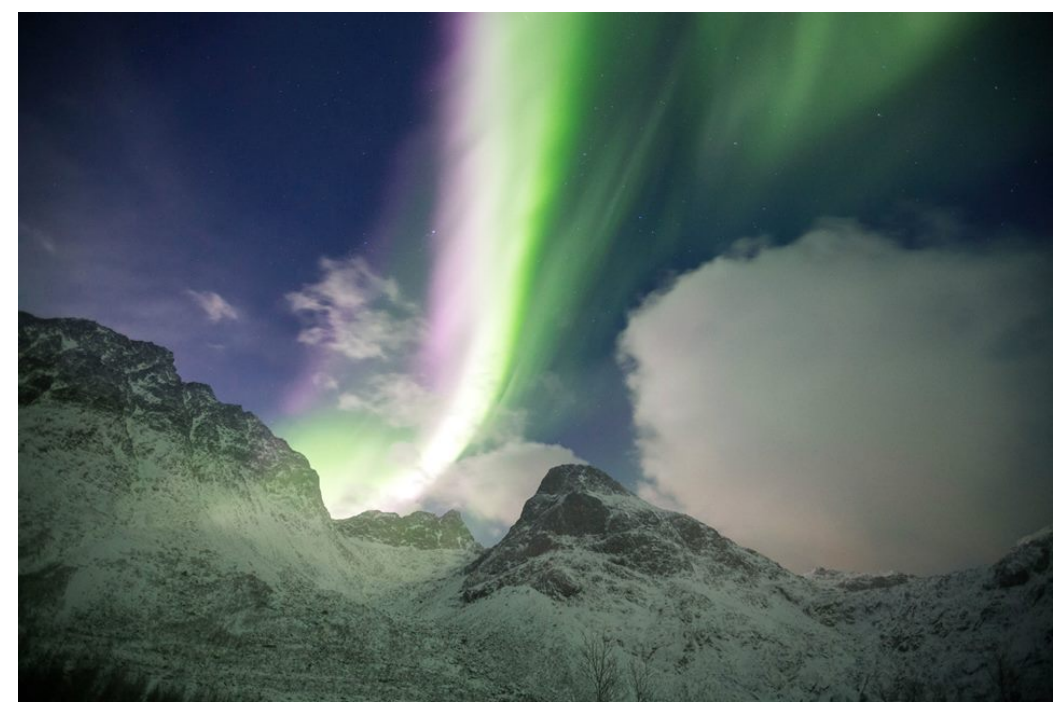

Fig. 1.6: Aurora Borealis taken on 14 December 2014 in Lofoten - Norway about 200 to $400 \mathrm{~km}$ above the Arctic Circle. (Gordon Schücker)

wavelengths. Molecular nitrogen radiates in blue and purple, atomic oxygen in green and red. Red auroras are observed at higher altitudes whereas green auroras are most common. Usually auroras are visible in both hemispheres at around $66.5^{\circ}$ geographic latitude and higher. During strong geomagnetic storms the auroras can be observed at latitudes as low as Spain or Hawaii. Auroras are not an exclusive Earth phenomenon as similar auroral activities have also been spotted on Mars (Bertaux et al., 2005) as well as on Jupiter, Saturn, Uranus, Neptune and Venus.

\subsection{Motivation and Objectives of this Work}

Due to the potential risk of CMEs for our society, space weather researchers all over world are trying to predict their arrival at Earth. It is known, that the Carrington CME possessed an average speed of $2400 \mathrm{~km} / \mathrm{s}$ and reached Earth in 17.6 hours (Cliver et al., 1990) and by comparison, the slowest known CMEs take up to 6 days. But until today terrestrial arrival predictions of CMEs are not very precise. The arrival time predictions of CME 
forecasters are frequently compared ${ }^{1}$. Their prediction accuracy varies between 20 hours for slow events and 6 hours for fast ones. A main reason of this large uncertainties are the difficult measurements of relevant initial CME and solar wind parameters. Besides knowledge about the prevailing solar wind conditions, the performance of approved CME propagation models relies on these measurements of the CME initial geometry and CME apex speed as well as the CME mass. For example, the Drag-Based Model (DBM) (Vršnak et al., 2013), which is based on the hydrodynamic analogue of the aerodynamic drag acting between the solar wind and the CME, depends on these properties. To maximise the benefit for space weather predictions, CME kinematics and geometry have to be determined as early as possibly after the CME emergence phase.

In this thesis, a main focus is laid on accurate parameterisations of CMEs. The determination of the CME kinematics and morphology is s very challenging, as it requires very precise measurements of the relevant CME parameters. Coronagraphs can provide these values with the downside that they only observe 2-dimension projections of a 3-dimensional object. Thus, the measurements are not very reliable as they suffer from projection effects. Such projection effects have often been discussed in the context of CME kinematics (Burkepile et al., 2004; Vršnak et al., 2007; Temmer et al., 2009; Shen et al., 2013), masses (Vourlidas et al., 2010) and morphologies (Vourlidas et al., 2013; Kwon et al., 2015; Cremades \& Bothmer, 2004). With the launch of NASA's Solar TErrestrial Relations Observatory (STEREO) mission in October 2006 (Kaiser et al., 2008), stereoscopic observations of CMEs have been made possible and enable to deproject the 3-dimensional structures of CMEs.

In this work, a combined coronagraph based parameterisation method is presented, which allows to measure all relevant CME parameters in a consistent and comparable manner. The method is applied to a set of 122 events with intense brightness, that were stereoscopically observed with the coronagraphs of Sun-Earth Connection Coronal and Heliospheric Investigation (SECCHI; Howard et al., 2008a) suite onboard of the twin STEREO spacecraft. The derived CME parameters are statistically analysed and mutually correlated.

The following scientific and methodological questions will be addressed in this work:

1. How can the morphology, the velocity and the mass of CMEs be measured from coronagraph observations?

2. Are such measurements reliable for stereoscopic observations?

3. What are typically measured CME properties and how are they connected to the solar activity?

4. How can the measurement of these properties improve CME forecasts?

\footnotetext{
${ }^{1}$ https://kauai.ccmc.gsfc.nasa.gov/CMEscoreboard/
} 


\subsection{Thesis Guideline}

Chapter 1 has so far given a short overview of the discovery of Coronal Mass Ejections and associated phenomena. As most important players in the terrestrial relationship, the research of CMEs and the necessity of accurate CME forecast was motivated by displaying documented impacts of space weather related effects at Earth. This was followed by a definition of the scientific goals of this thesis.

Chapter 2 gives a brief introduction of the fundamental structure of the Sun and physical concepts governing its behaviour with a special focus on the outer atmospheric layers - the birthplace of CMEs.

Chapter 3 follows with a description of the heliosphere, the solar wind, the interplanetary magnetic field and Earth's magnetosphere.

Chapter 4 highlights the formation, structure and triggering mechanisms of CMEs. Further, their causal relationship to other associated coronal phenomena, namely solar flares, eruptive prominences and coronal shock waves, is discussed.

Chapter 5 presents the instruments and spacecraft used for the observation of CMEs in detail as well as relevant heliocentric coordinate systems.

Chapter 6 treats the manifestation of CME white-light structures, which is based on the Thomson scattering mechanism. It further discusses projection effects related to the detected CME brightness and implications for the CME mass determination.

Chapter 7 focuses on the geometrical modelling of CME white-light structure with the help of stereoscopic coronagraph observations. To this end the applied GCS model and the fitting procedure are described in detail. Implications on stereoscopic GCS fitting are further discussed based on the fitting results of ten sample events.

Chapter 8 starts with a theoretical foundation of CME mass determination from coronagraph white-light images. This is followed by the presentation of a new combined method of geometrical CME modelling with the Graduated Cylindrical Shell model and CME mass determination technique. The method is applied to a set of $122 \mathrm{CME}$ events. The results are discussed in terms of CME statistics and projection effects.

Chapter 9 demonstrates potential application of this results in real time CME forecast. A CME propagation model is proposed, which combines a heliospheric kinematic model the Drag-Based model with a geometrical model - the Graduated Cylindrical Shell model. Further, different heliospheric propagation scenarios of a sample event and their connection to solar wind parameters as well as the CME mass are discussed.

Chapter 10 summarises the most notable results and answers the scientific research questions of this thesis. Finally, an outlook onto relevant implications for future CME parameterisation is given. 



\section{The Sun}

In this chapter the fundamental physics and concepts of the Sun and its inner structure are presented. The atmospheric layers are the "birthplace" of CMEs and are therefore discussed in more detail together with some illustrative images. A special focus is laid on the most outer layer, the solar corona, their physics and observable phenomena within. This is followed by a discussion of the solar magnetic field and its connection to the solar cycle.

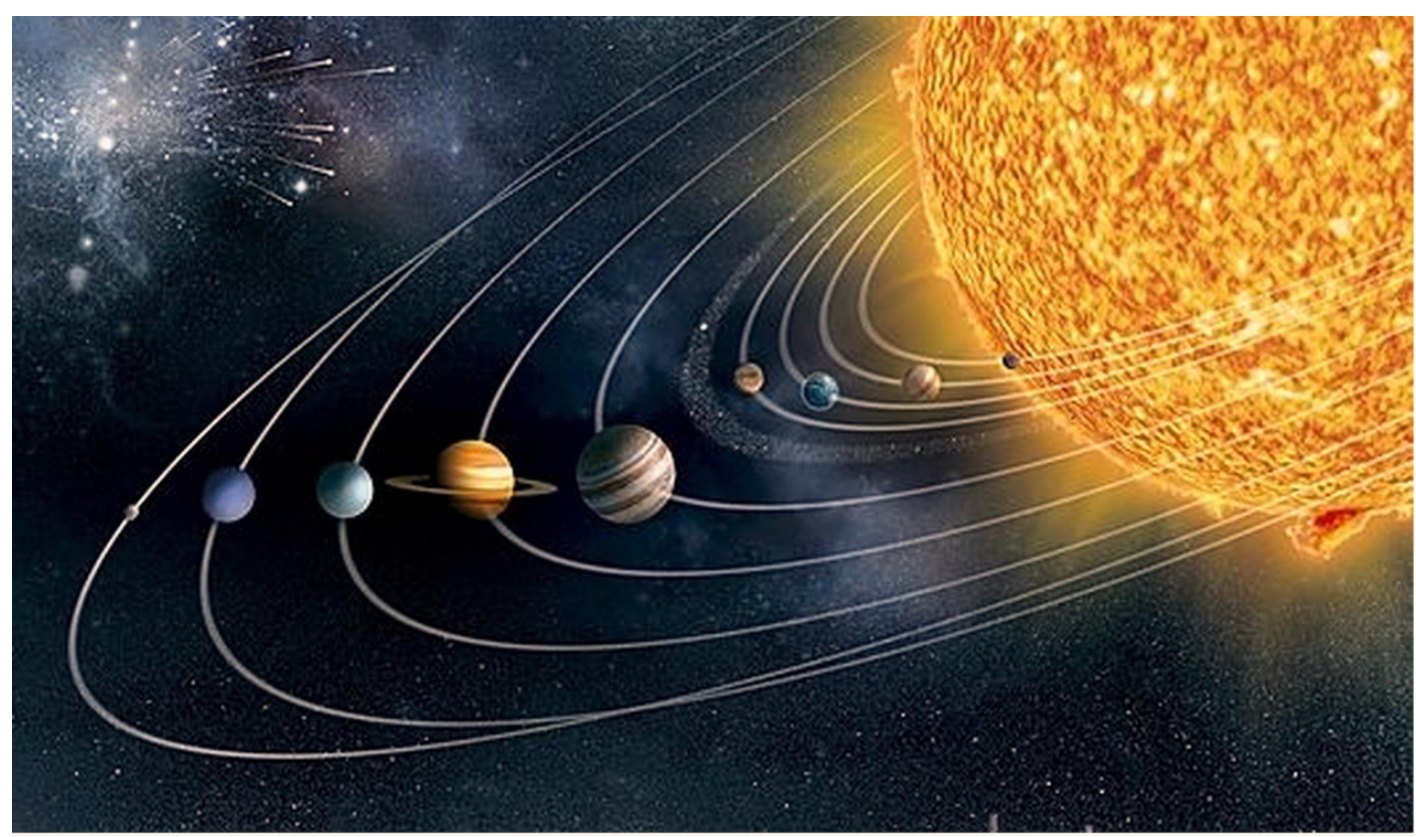

Fig. 2.1: Artistic illustration of the solar system. (NASA - Marshall Spaceflight Center)

The Sun is a yellow main sequence dwarf star (G2V-type) in the heart of our Solar System (see Figure 2.1). With a radial size of $696,342 \mathrm{~km}$, the Sun is 109 times larger than Earth. The average distance from the Sun to Earth of $1.49 \times 10^{11} \mathrm{~km}$ is defined as an astronomical unit (AU). Emitted solar photons travel this distance in about 8.3 minutes. The Sun has a synodic solar rotation period of around 27.25 days at the prime median, defined as one Carrington Rotation. The mass of the Sun, $1.9891 \times 10^{30} \mathrm{~kg}$, is about 330,000 Earth masses and represents $99.86 \%$ of the total mass in the solar system. It is mainly composed of hydrogen $(\sim 73 \%)$, helium $(\sim 25 \%)$ and small quantities of heavier elements including oxygen, carbon, neon and iron (Basu \& Antia, 2008). Like other stars, the Sun is a giant ball of high-temperature plasma with a luminosity of $L_{\odot}=3.84 \times 10^{26} \mathrm{~W}$. It's tremendous energy release amounts to $3.8 \times 10^{26} \mathrm{~J}$ per second. The energy itself is generated by nuclear fusion of hydrogen nuclei in the inner solar core. The evolution of the Sun (Figure 2.2) began with the gravitational collapse of an interstellar molecular cloud around 4.6 billion years ago (Connelly et al., 2012). In less than 1 million years the molecular cloud started 
the hydrogen nuclear fusion (Bouvier \& Wadhwa, 2010; Montmerle et al., 2006) and formed the Sun as we know it. In around 5 billion years the supply of hydrogen in the solar core will exhaust and thermonuclear fusion will start in a shell surrounding the core. Since the Sun leaves its hydrostatic equilibrium, the core will increase in density and temperature while the outer layers expand. As the core gets hotter, helium becomes the new fuel in the core. At the end of this stage the Sun will have grown to a radius of $166 R_{\odot}$, which means that it will incorporate the planets Mercury and Venus. In around 12.5 billion years the Sun will have lost about half of its mass as it sheds its outer layers. When almost all of its fuel is used up, the Sun will have shrunk into a white dwarf with the approximate size of Earth.

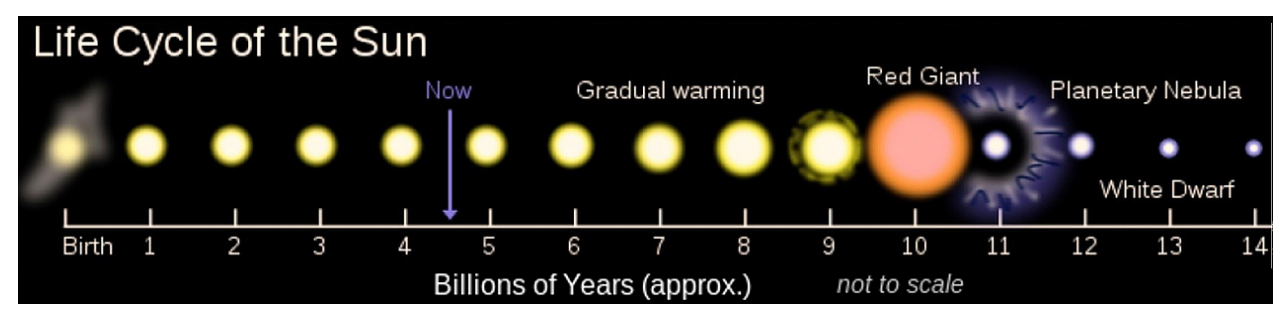

Fig. 2.2: The Evolution of the Sun from a interstellar molecular cloud to a yellow dwarf, a red giant and finally to white dwarf. (Oliver Beatson.)

\subsection{The Solar Interior}

The solar interior is separated into four regions, each dominated by a different energy transport mechanism. The Sun's energy is produced in the Solar Core, the innermost region with a size of $\sim 0.25 \mathrm{R}_{\odot}$. The extreme ambient conditions - pressures of up to 265 billion bar and temperatures of up to 15 million kelvin - permit nuclear fusion of hydrogen nuclei into helium cores. The Radiative Zone is the next outer region. It spans from $0.25-0.7 \mathrm{R}_{\odot}$ and is characterised by its energy transport through thermal radiation. A temperature drop from seven to two million kelvin with increasing distance from the core is the consequence. Equally, the density drops from $20 \mathrm{~g} / \mathrm{cm}^{3}$ (the density of gold) to $0.2 \mathrm{~g} / \mathrm{cm}^{3}$ (less than the density of water). The high initial density in the radiative zone results in a very small mean free path for photons. Hence, it takes between 100.000 and 50 million years for a photon to escape to the next outer region, called tachocline. The Tachocline is a very sharp border between the radiative zone and the non-solid convective zone. Here, the rigid core rotation $(\sim 27$ days $)$ transforms to a latitudinal differential rotation resulting in a shorter rotation period at the equator (25.6 days) and longer rotation periods at the poles (33.5 days). In the Convective Zone the temperature drops from two million to around $5700 \mathrm{~K}$. As a result, heavier elements are no longer fully ionised, so that the radiative heat transport becomes less effective. The lower gas density in this region allows thermal convection, which dominates the outward heat transport.

\subsubsection{Differential Rotation}

Turbulences in the convective zone are creating helioseismic waves, forcing the Sun to oscillate at a characteristic eigenfrequency of $3.33 \mathrm{mHz}$, often termed as "five-minute oscillation". This oscillation can be observed by measuring the Doppler shift of atmospheric absorption lines. The method is used in helioseismology to study the internal structure of the Sun. Michelson Doppler Interferometer onboard the SOHO spacecraft or as part of the 


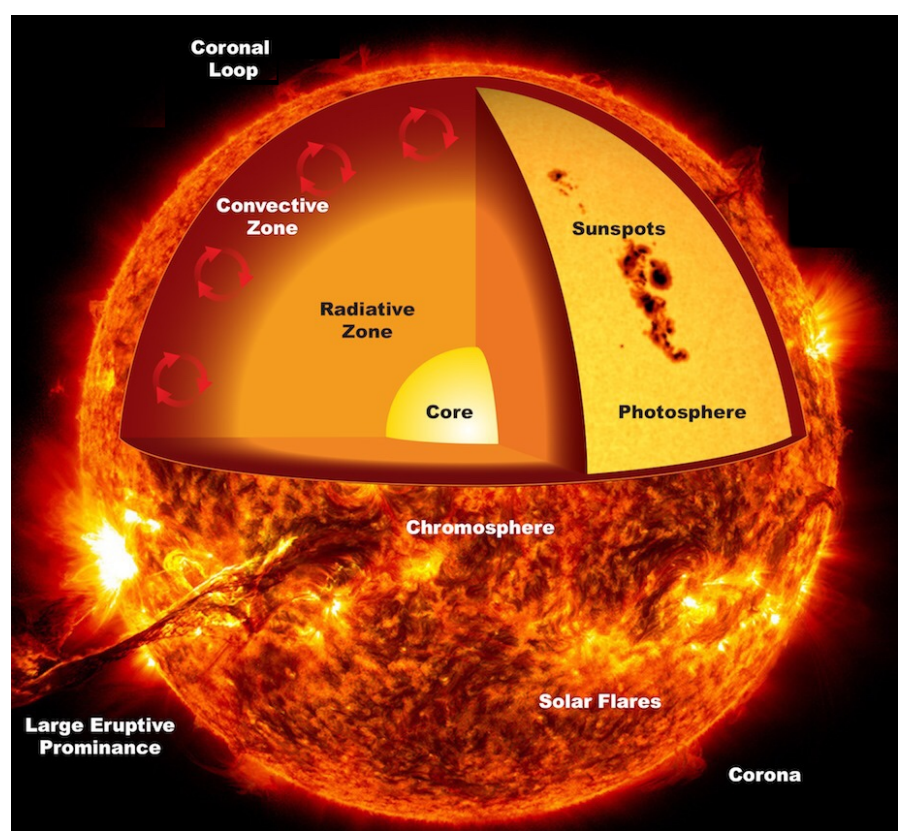

Fig. 2.3: Structure, features and inner working of the Sun from the inside to the outside solar core, radiative zone, convective zone, photosphere with sunspots, chromosphere and corona together with several transient phenomena. (NASA - Marshall Spaceflight Center)

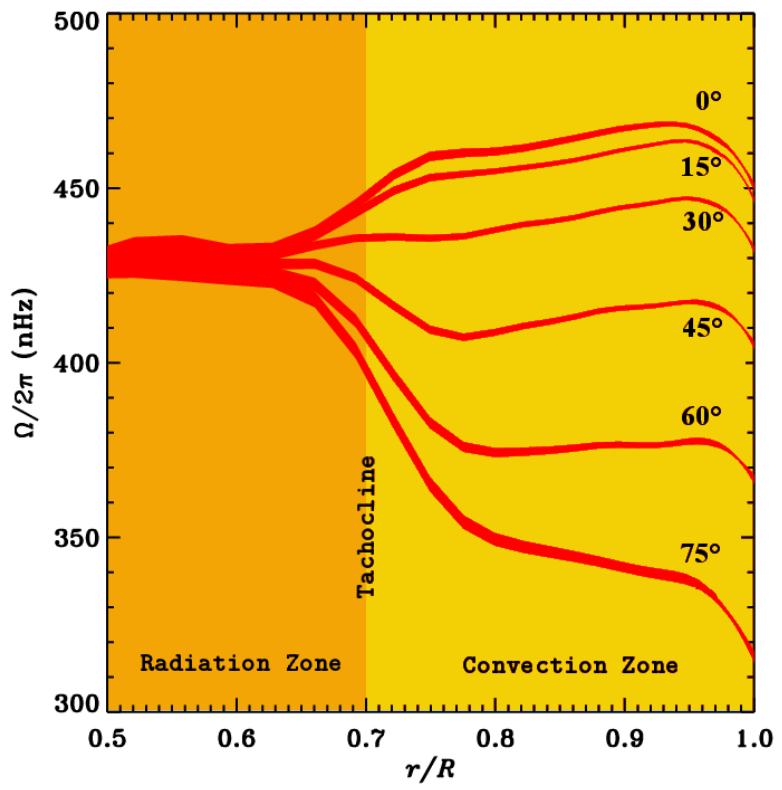

Fig. 2.4: Angular velocity profile for the solar interior rotation is plotted as a function of the fractional solar radius, at selected latitudes based on helioseismology (after Thompson et al. 2003). The data are averaged over the period 1995-2009. (Adapted from: National Solar Observatory/GONG) 
Global Oscillation Network Group (GONG) instrument (Harvey et al., 1996) are using this method to measure the radial and latitudinal dependence of the internal angular velocity, $\Omega=\Omega(r, \theta)$ throughout the Sun (Figure 2.4).

\subsection{The Solar Atmosphere}

The solar atmosphere shows a huge complexity of structures and dynamics as a result of the interaction between magnetic fields and solar plasma. It is composed of several layers, starting from the inside - photosphere, chromosphere, transition region and corona. As demonstrated in Figure (2.5), the particle density is steadily decreasing towards higher layers. Interestingly, the atmospheric temperature jumps from around $5000 \mathrm{~K}$ in the photosphere to a few million degrees in the corona in a small zone called transition region, a fact which was not realised until 1940.

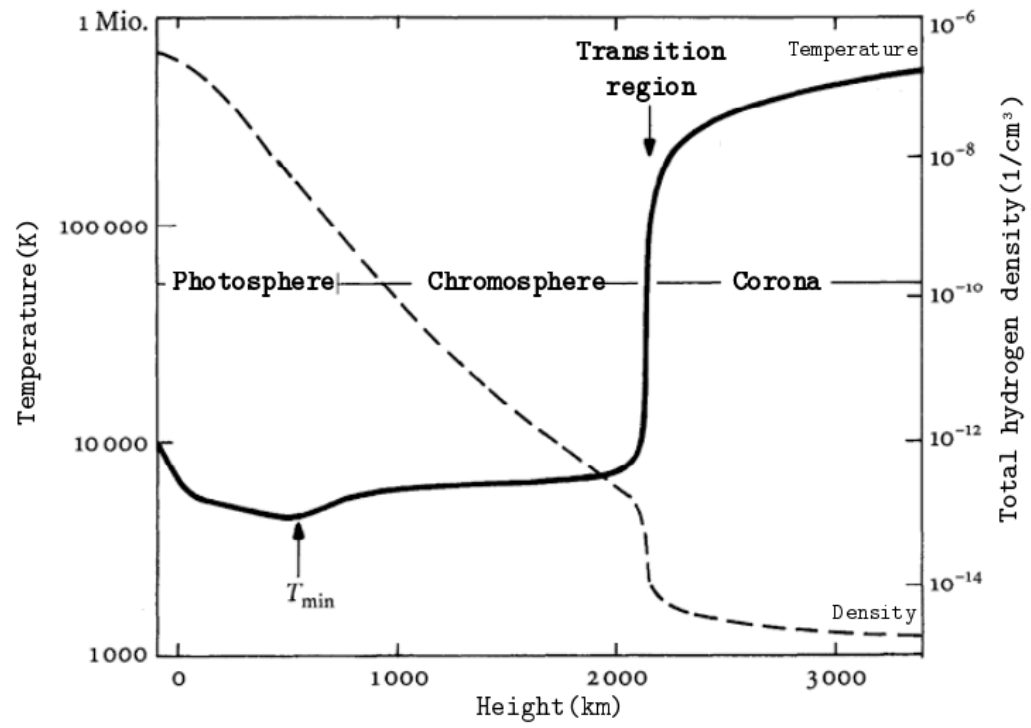

Fig. 2.5: Temperature and density distribution in the solar atmosphere. The atmospheric temperature abruptly rises in the transient region from a few thousand to nearly one million Kelvin. (Eugene Avrett, Smithsonian Astrophysical Observatory)

The Sun emits light over a broad spectral range (see Figure 2.6) with yellow as the brightest wavelength emitted in the visible spectrum. For scientific purposes, telescopes like NASA's Solar Dynamics Observatory (SDO), NASA's Solar Terrestrial Relations Observatory (STEREO) and the ESA/NASA Solar and Heliospheric Observatory (SOHO) examine the Sun at many different wavelengths, some of which are in the extreme ultraviolet and $\mathrm{x}$-rays spectrum. This light originates from atoms and ions of the solar atmosphere. They are emitting light in a specific wavelength as they reach certain temperatures. Spectral bandpass filters can therefore be used to highlight different atmospheric layers of the solar atmosphere. This is demonstrated in Figure 2.7 with images taken by the Atmospheric Imaging Assembly(AIA; Lemen et al. 2012) and the Helioseismic and Magnetic Imager (HMI; Scherrer et al. 2012) aboard of the Solar Dynamics Observatory (SDO; Pesnell et al. 2012). 


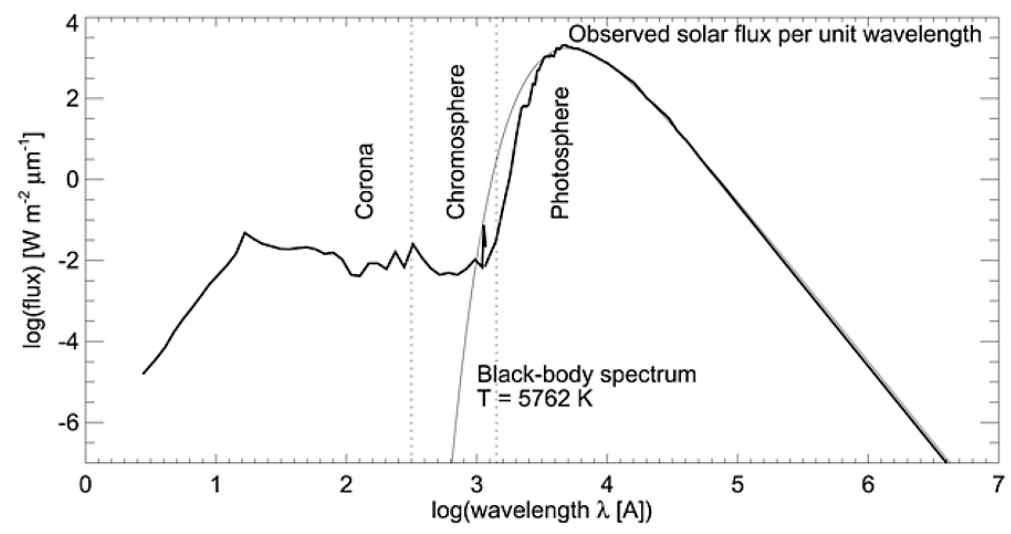

Fig. 2.6: Measured solar irradiation spectrum compared to a black-body with $\mathrm{T}=5762 \mathrm{~K}$. (Aschwanden, 2004).

The Photosphere is the lowest atmospheric layer of a star and is transparent for photons of the visible wavelength. Most of the emitted light comes from this layer with a thickness of around $500 \mathrm{~km}$. Its particle density of $10^{23} \mathrm{~m}^{-3}$ is much higher compared to the chromosphere $\left(10^{17} \mathrm{~m}^{-3}\right)$ and the corona $\left(10^{15} \mathrm{~m}^{-3}\right)$. The visual appearance of the photosphere is characterised by bright granules of plasma and darker cooler sunspots (see Figure 2.8, 2.9 and 2.10). Hot plasma is rising in tubular streams to the solar surface, driven by a high temperature gradient in the convection zone, spanning from two million to 5700 Kelvin. This effect creates convection cells, so called granules, which have a typical size scale of $1000 \mathrm{~km}$. The colder plasma sinks between the narrow spaces and after minutes the different temperatures of these upward and downward streams cancel each other out. Typical speeds in granular flows are in the order of a few kilometres per second. The short granule lifetime of 5-10 minutes creates a visual "boiling" pattern.

The Chromosphere is the birthplace of many solar phenomena, like filaments, spicules and solar flares. It extends from 3,000 to $5,000 \mathrm{~km}$ above the solar surface and has only $10^{-4}$ times the density of the photosphere. During lunar eclipses, the chromosphere can be seen as a pale red shell above the photosphere (see Figure 2.11). Outside of eclipses, the chromosphere is invisible in the white light spectrum due to the much brighter photosphere. In contrast to the absorption line spectrum, the chromosphere is dominated by emission lines. The characteristic red glow (compare with Fig 2.14) is caused by the $\mathrm{H}_{\alpha}$ spectral line of excited hydrogen at a wavelength of $656 \mathrm{~nm}$. Interference filters like the FabryPérot-interferometer can be used to study the structure of the chromosphere. It reveals a Chromospheric Network - a web-like pattern formed by magnetic field lines best visible in the $\mathrm{H}_{\alpha}$-spectrum (see Figure 2.12).

The Solar Transition Region between the cold chromosphere and the much hotter corona (see Figure 2.13$)$ is extremely narrow $(\sim 200 \mathrm{~km})$. Nevertheless, it is of great relevance as it marks an important changing point in the physical behaviour of the solar atmosphere. Below the solar transition region, gas pressure, fluid dynamics and gravitation are responsible for every structure formation - above, all motion and structure follows the laws of magnetohydrodynamics. Instead of hydrogen, which is fully ionised, the light emitted by the transition region is dominated by ions of carbon, oxygen, and silicon. The sharp temperature gradient in the transition region results from the full ionisation of helium. In this state, cooling via black body radiation or direct coupling to the helium Lyman continuum is not efficient. This leads to an abrupt rise of the equilibrium temperature to nearly one million kelvin. 


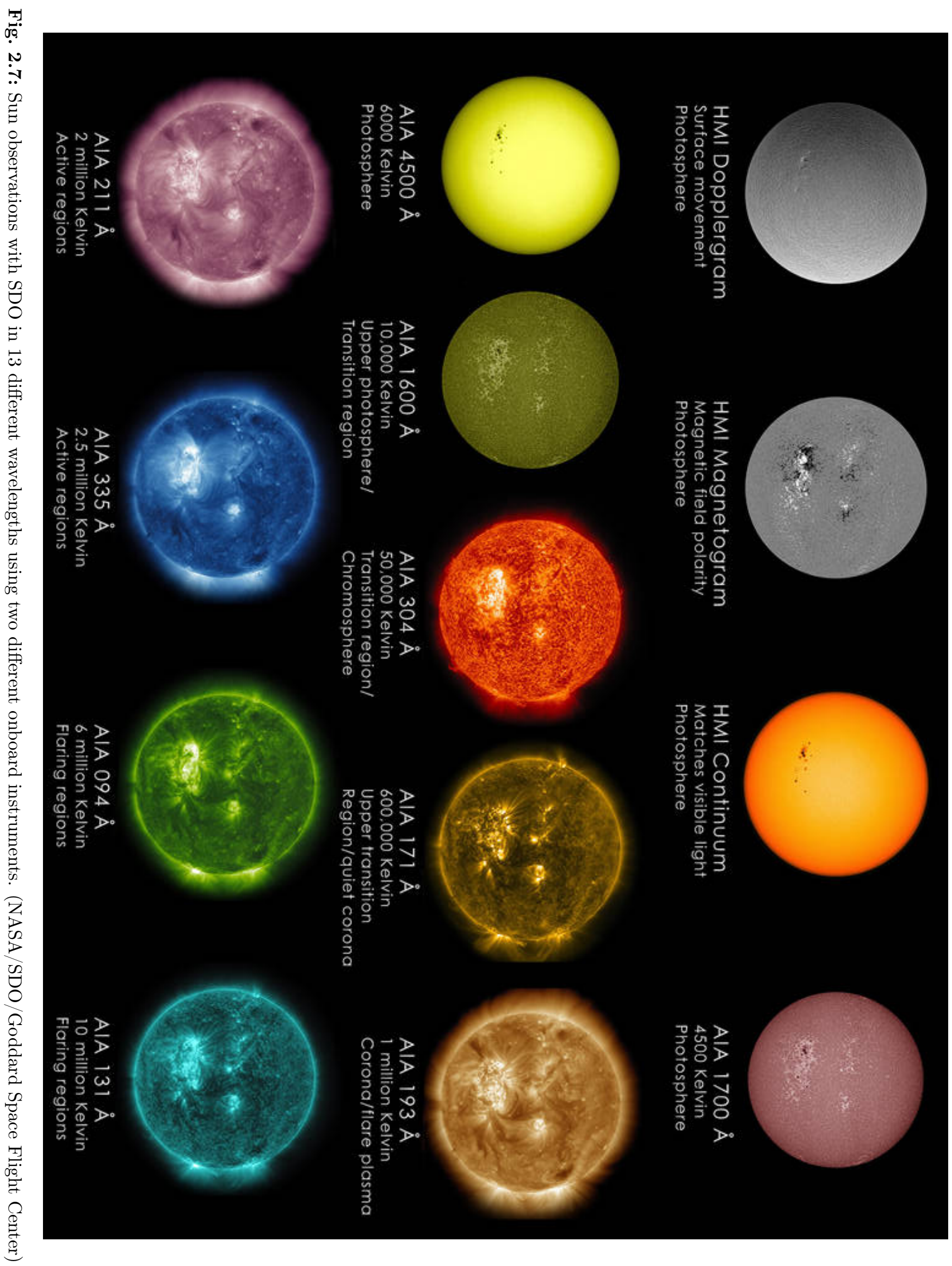




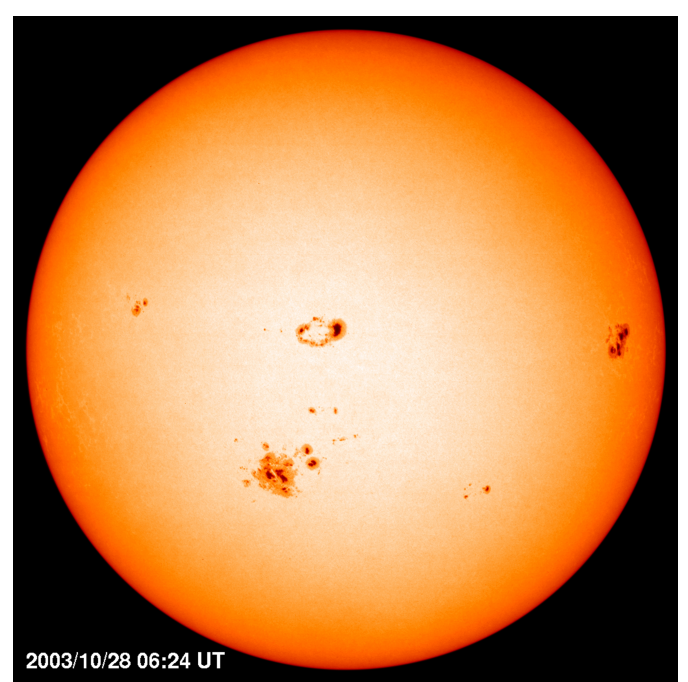

Fig. 2.8: Photosphere with sunspots observed with $\mathrm{SOHO} / \mathrm{MDI}$ Continuum. (SOHO NASA)

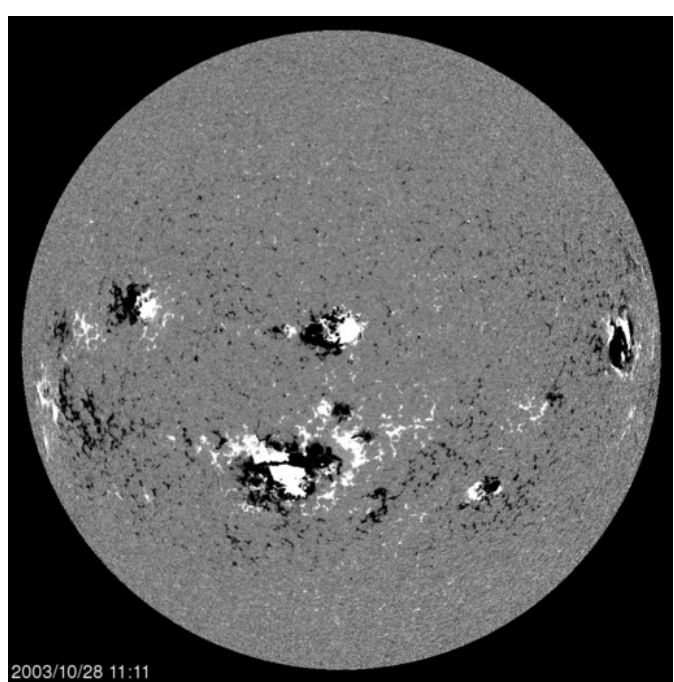

Fig. 2.9: Photosphere with sunspots observed with $\mathrm{SOHO} / \mathrm{MDI}$ Magnetogram. (SOHO NASA)

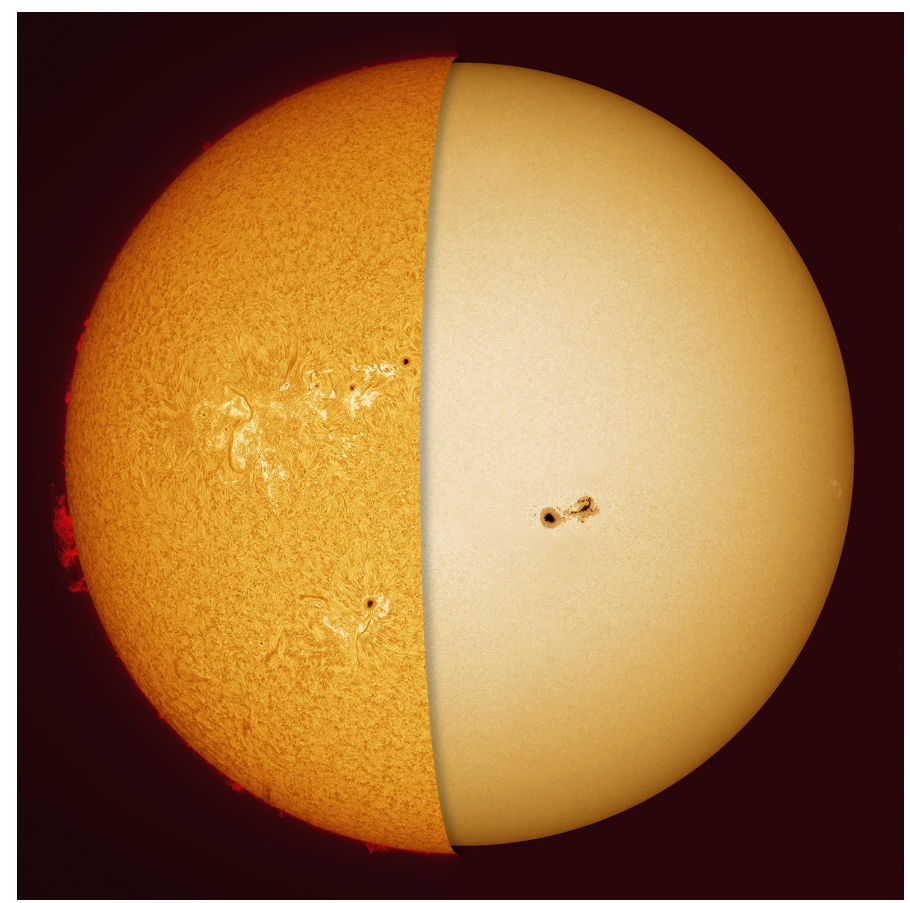

Fig. 2.10: The Sun captured with the Little Big Man, PGR Grasshopper Express 6 megapixel camera, on September 23, 2012. On the right, the photosphere in the white light spectrum reveals sunspots and granulation structures. On the left, the solar photosphere in the spectrum of $\mathrm{H}_{\alpha}$ $(656.3 \mathrm{~nm})$ with filaments and hot active regions. In the narrow slice deep in the red, the solar chromosphere is captured. (Alan Friedman) 


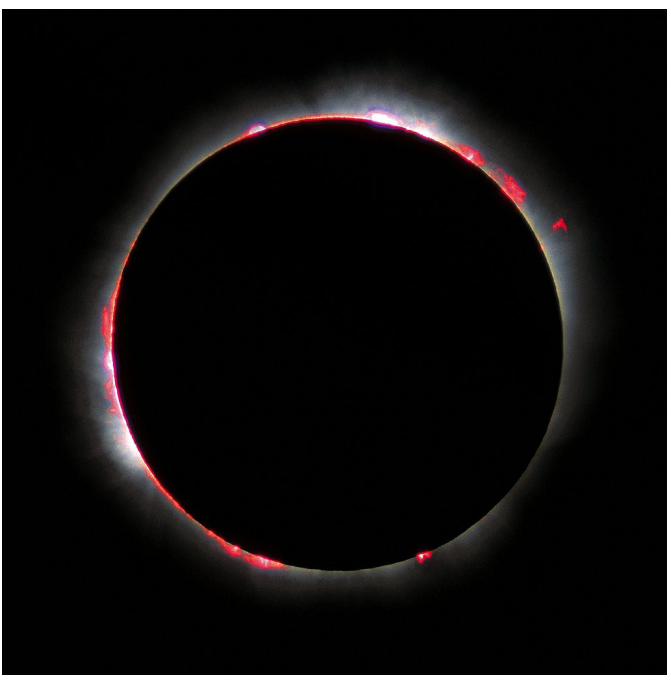

Fig. 2.11: Chromosphere seen in the visible spectrum during the solar eclipse on 11 August 1999 in France. (Luc Viatour)

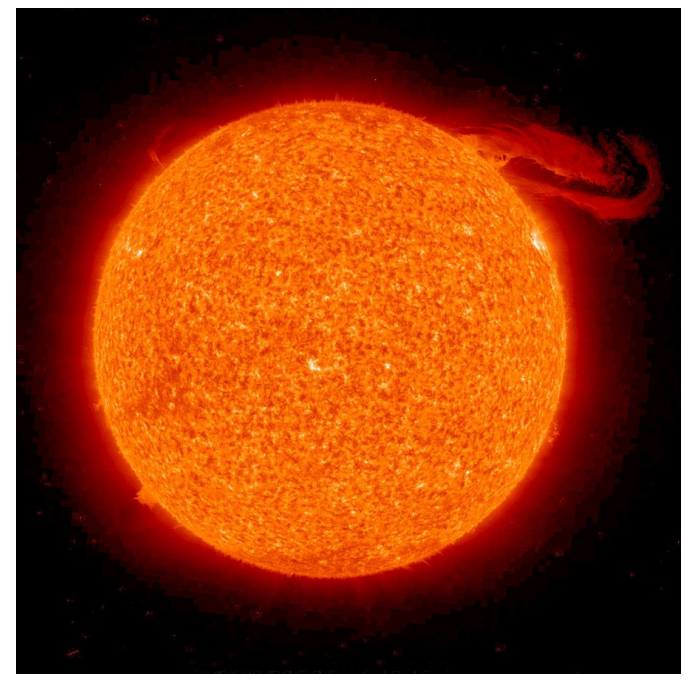

Fig. 2.12: Chromosphere observed by STEREO A in $304 \AA$ wavelength of extreme UV light on 29 September 2008. At the top right a prominence, composed of ionised helium, emerges. (NASA)
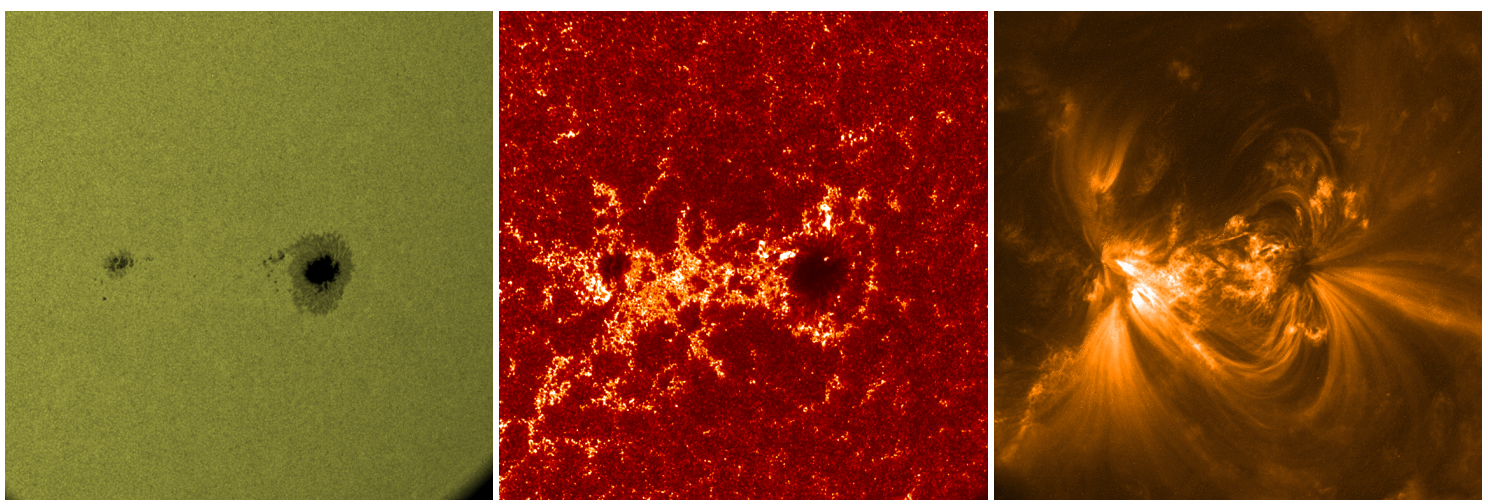

Fig. 2.13: Images of the active region 9017 for three different solar atmosphere layers taken by the Transition Region And Coronal Explorer (TRACE) on June, 2000 at 14.01 UT. The different layers and the observed wavelengths named from left to right: the photosphere (white-light), the transition region $(1600 \AA)$ and the corona $(171 \AA)$. The active region manifest as sunspots in the photosphere and as a set of magnetic loops in the corona. (Dawn Myers) 


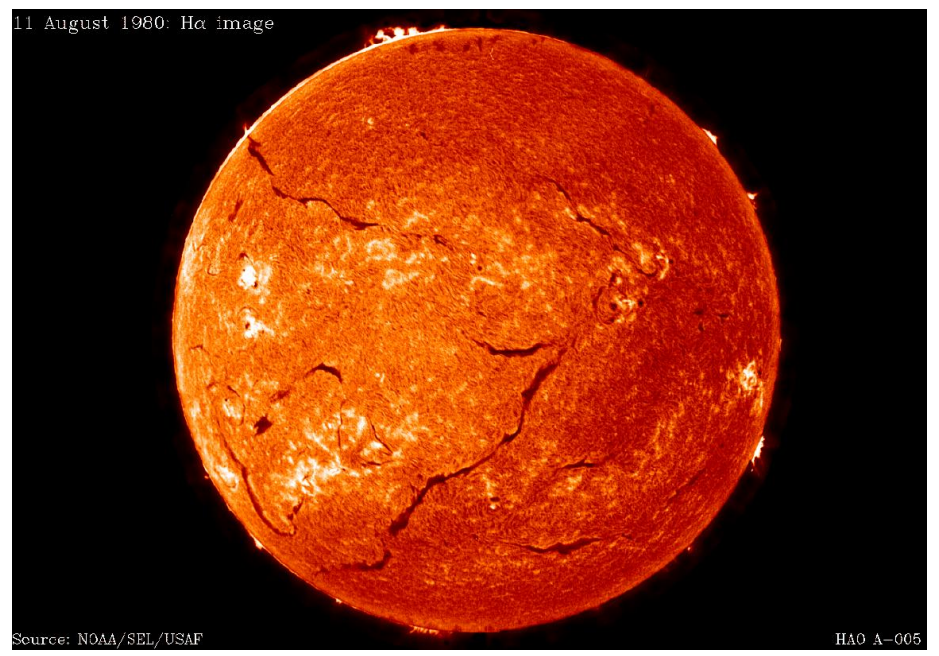

Fig. 2.14: Filament lines in the chromosphere observed in $\mathrm{H}_{\alpha}$ on 11th August 1980 with Courtesy High Altitude Observatory (HAO) of the Nation Center for Atmospheric Research (NCAR). (National Oceanic and Atmospheric Administration - NOAA / United States Air Force - USAF)

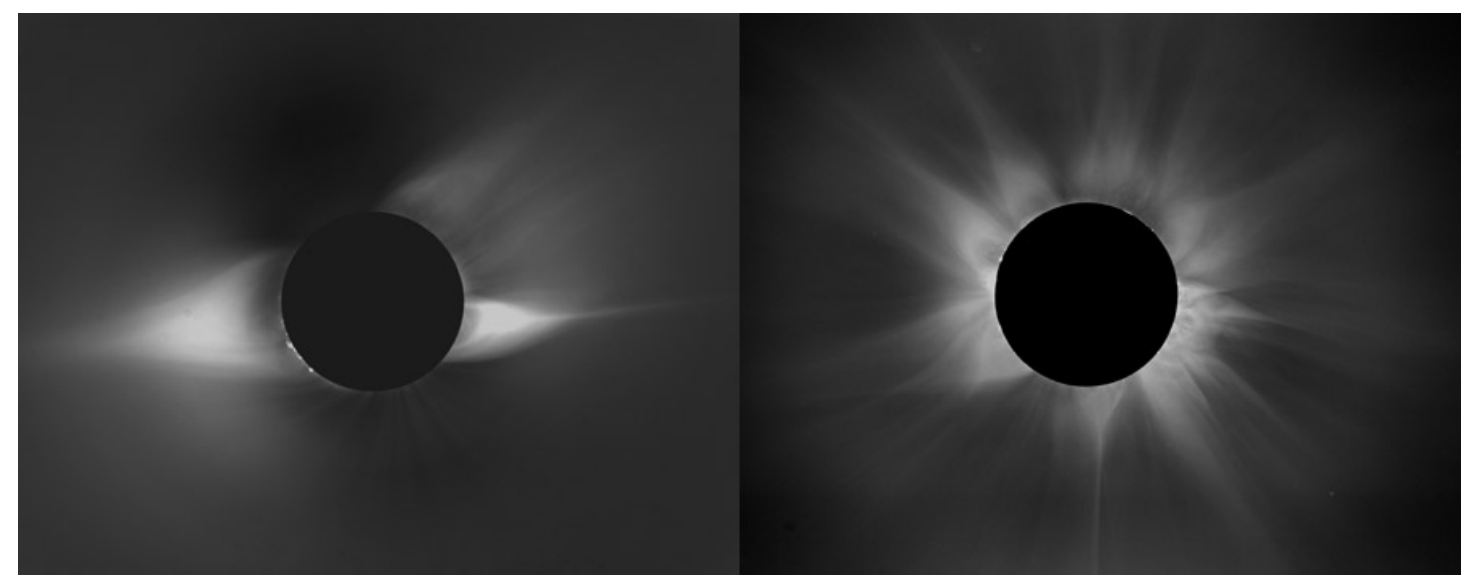

Fig. 2.15: Solar corona around the solar cycle minimum seen during the total eclipse on November 3, 1994 (left) and during the solar maximum on February 16, 1980 (right). (Bothmer \& Daglis 2007 - Courtesy: High Altitude Observatory, Boulder, CO)

The Solar Corona starts at a height of $\sim 2100 \mathrm{~km}$ above the solar surface and stretches from the transition region to around $12-15 \mathrm{R}_{\odot}$ (DeForest et al., 2014). The corona consists of fully ionised hydrogen and helium of temperatures between one and three million Kelvin, which is much hotter than the photosphere. Due to its $10^{-12}$ times lower density, it emits $10^{-6}$ times less visible light than the photosphere, and therefore can only be observed with the naked eye during a total solar eclipse or with the help of coronagraphs. Corona translates from Latin into the word "crown" and alludes to its white-light appearance during maximal solar activity. The observable corona consists of magnetically formed large-scale structures (see Section 2.19), namely helmet-streamers and coronal loops. These structures are controlled by the solar magnetic field, which can largely fluctuate on the timescale of hours. The global shape of the solar corona differs dramatically during periods of maximal and minimal solar activity (Figure 2.15) as the solar magnetic field transforms from a pure dipole into a more complex configuration. Most of the radiation emitted by the corona lies in the X-ray and part of the ultraviolet spectrum (Figure 2.16). Ultraviolet observations reveal the abundance of large-scale structures. Combined observation of different 

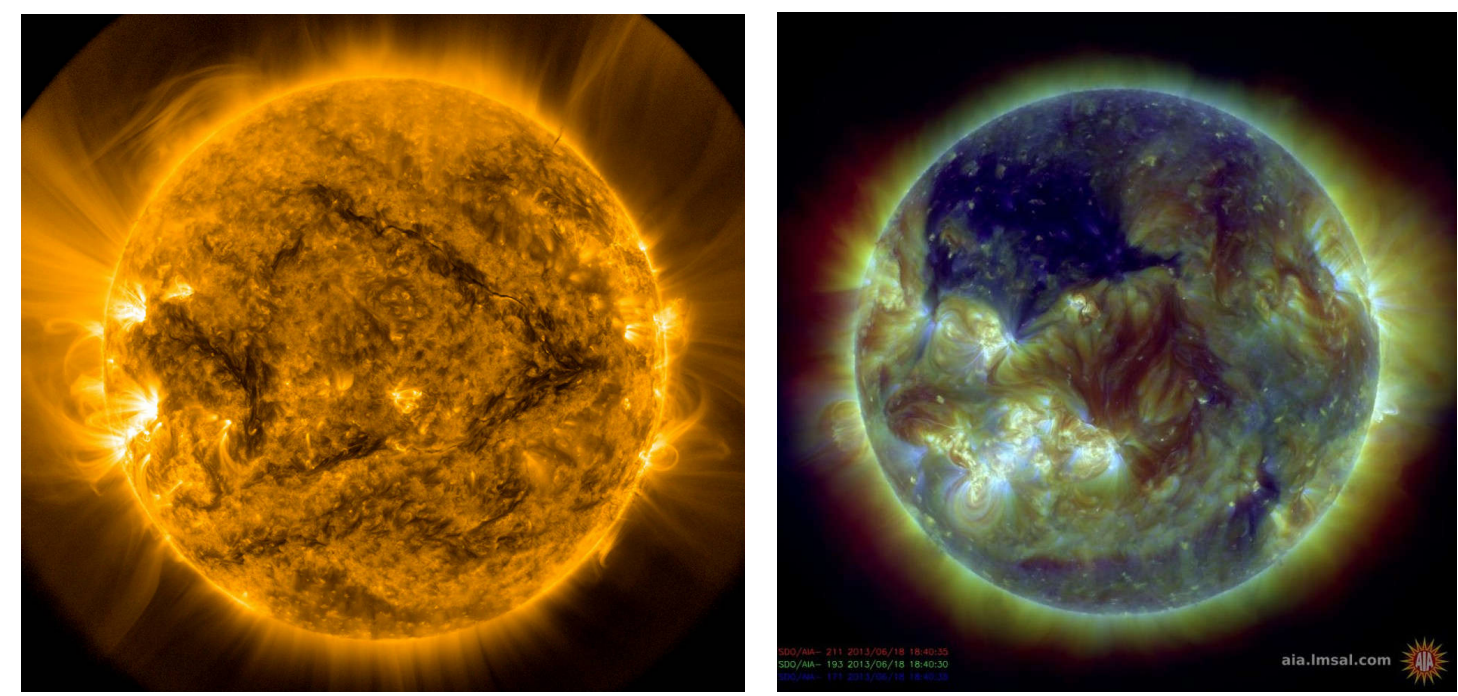

Fig. 2.16: Solar corona captured at $171 \AA$ shows coronal plasma at a temperature of about a million kelvin. An active region with intense emission is visible on the left. The image was taken with the Atmospheric Imaging Assembly (AIA) instrument of Solar Dynamics Observatory on May 5, 2015. (NASA/SDO)
Fig. 2.17: Solar corona observed in a combination of different UV spectra recorded by the Atmospheric Imaging Assembly (AIA) instrument of Solar Dynamics Observatory on June 18, 2013. At the equator an abundance of coronal loops embedded in active regions is visible. Also a huge coronal hole has manifested in the northern polar region. (NASA/SDO)

wavelengths can also reveal coronal holes (Figure 2.17). Coronal holes are formed at wide unipolar regions of the solar magnetic field. The biggest coronal holes are generally observed at the poles during solar minimum. Many different sources contribute to the visible spectrum of the corona. The main contribution comes from scattering of photospheric radiation by free electrons, called the K-corona (kontinuierlich, German for "continuous), as well as by dust in the interplanetary space, called F-Korona (Frauenhofer). Above $4 R_{\odot}$, the F-Corona starts to dominate over the K-Corona. Further known intensity contributions come from the E-Corona (emission) by the emission from highly ionised coronal atoms and the T-Corona (thermal) by the infrared emission of the interplanetary dust. One peculiarity of the corona, the heating of the corona by the photosphere, is still controversially discussed. The second law of thermodynamics in principle forbids the flow of heat from a cooler heating source (photosphere) to a hotter heating target (corona). Therefore, it is obvious that energy must be carried to the corona by some non-thermal means. Current theories are constructed around damped hydromagnetic waves and reconnection (Malara \& Velli, 2001) or nanoflares (Klimchuk, 2006). All of these theories are associated with the magnetohydrodynamic nature of the corona, which shall be considered more closely in the following section. 


\subsection{The Physics of Coronal Plasma}

\subsubsection{Magnetohydrodynamics}

The field of magnetohydrodynamics (MHD) was strongly influenced by Hannes Alvén, who was rewarded with the Nobel Price in 1970. The theory describes the hydrodynamics of magnetised fluids (e.g. salt water, liquid metals, plasma) through the combination of electrodynamics and fluid dynamics. The dynamics of the coronal plasma can be physically described by the theory of magnetohydrodynamics.

MHD is based on the fundamental concept that a moving conductive fluid in a magnetic field induces currents, which in the presence of the magnetic field, generates a force that acts on the motion of the fluid. In return, the fluid motion alters the geometry and strength of the magnetic field itself. The main quantities which characterise such electrically conductive fluids are the bulk plasma velocity $\boldsymbol{v}$, the current density $\boldsymbol{j}$, the mass $m$, the mass density $\varrho$, the plasma pressure $p$, the magnetic Field $\boldsymbol{B}$ and the electric field $\boldsymbol{E}$.

\section{Lorentz Force}

In magnetised plasma, the Lorentz Force $\boldsymbol{F}_{l}=q \boldsymbol{v} \times \boldsymbol{B}$ is effective on a particle with a charge $q$. The particles move on trajectories perpendicular to the magnetic field, with the Larmor radius $r_{L}$ and cyclotron frequency $\omega_{c}$. They are defined as

$$
r_{L}=\frac{m v_{\perp}}{|q| B}
$$

and

$$
\omega_{c}=\frac{|q| m}{B} .
$$

\section{Conditions for the Application of Magnetohydrodynamics}

The theory of magnetohydrodynamics describes the large-scale, slow dynamics of plasmas and can be applied when:

1. The characteristic time $T$ is much greater than the ion gyroperiod and mean free path time of the system:

$$
\frac{1}{\omega_{c}} \ll T .
$$

2. The characteristic length $L$ is much greater than the ion gyroradius and the mean free path length of the system:

$$
r_{L} \ll L .
$$

3. The plasma velocities $v$ are not relativistic:

$$
v \ll c .
$$

In the following the theory of magnetohydrodynamics is derived from the combination of electrodynamics and fluid theory. 


\section{Maxwell's Equations}

Maxwell's equations form the foundation of electromagnetism:

$$
\begin{aligned}
& \nabla \times \boldsymbol{B}=\mu_{0} \boldsymbol{j}+\varepsilon_{0} \mu_{0} \frac{\partial \boldsymbol{E}}{\partial t}, \\
& \text { (Ampere's law) } \\
& \nabla \cdot \boldsymbol{B}=0, \\
& \text { (Gauss's law for magnetism) } \\
& -\nabla \times \boldsymbol{E}=\frac{\partial \boldsymbol{B}}{\partial t}, \\
& \text { (Faraday's law) } \\
& \nabla \cdot \boldsymbol{E}=\frac{\varrho}{\varepsilon_{0}} . \\
& \text { (Gauss's law) }
\end{aligned}
$$

Together with the Lorentz Force, they fully describe all classical phenomena of electrodynamics.

\section{Equation of State}

Regarding fluid dynamics, the plasma pressure $p$ can be determined from the mass density and the plasma temperature $T$ by the equation of state (e.g. the ideal gas law). For a pure hydrogen plasma this equation is given as

$$
p=2 \frac{\mathrm{k}_{\mathrm{B}}}{\mathrm{m}_{\mathrm{p}}} \varrho T
$$

where $\mathrm{m}_{\mathrm{p}}$ is the proton mass and $\mathrm{k}_{\mathrm{B}}$ the Boltzmann's constant. The equation of state of the system is then given by

$$
\frac{d}{d t}\left(\frac{p}{\varrho^{\gamma}}\right)=0
$$

where $\gamma$ is the polytropic index and defined as the ratio of the specific heats $C_{p} / C_{V}$, where $C_{p}$ is the heat capacity at constant pressure and $C_{v}$ is the heat capacity at constant volume. It is taken as $5 / 3$ in the adiabatic case.

\section{Mass Continuity Equation}

The fundamental equation of fluid dynamics is the mass continuity equation,

$$
\frac{\partial \rho}{\partial t}+\nabla(\varrho \boldsymbol{v})=0
$$

stating that mass is neither created nor destroyed.

\section{Momentum Equation}

The motion of the plasma is described in hydrodynamics by the momentum equation, also known as Euler's equation which is a special case of the Navier-stokes equation for nonviscous elastic fluids. To account for magnetohydrodynamics, it is expanded by a Lorenz force term to:

$$
\varrho \frac{\partial \boldsymbol{v}}{\partial t} \varrho(\boldsymbol{v} \cdot \nabla) \boldsymbol{v}=-\nabla p+\boldsymbol{j} \times \boldsymbol{B} .
$$

Additional viscosity terms can be neglected, because any transport process perpendicular to the magnetic field is strongly inhibited by the gyration whereas advection dominates 
along field lines. The field equation for $\boldsymbol{B}$ can be derived from the first Maxwell equation (Eq. 2.6). In the MHD-approximation the second term, called displacement current, can be neglected, yielding:

$$
\nabla \times \boldsymbol{B}=\mu_{0} \boldsymbol{j} .
$$

The vector identity

$$
(\boldsymbol{a} \cdot \nabla) \boldsymbol{a}=\nabla\left(a^{2} / 2\right)+(\nabla \times \boldsymbol{a}) \times \boldsymbol{a}
$$

can now be used to eliminate the current and replace it with the magnetic field.

$$
\boldsymbol{j} \times \boldsymbol{B}=\frac{1}{\mu_{0}}(\nabla \times \boldsymbol{B}) \times \boldsymbol{B}=-\nabla\left(\frac{B^{2}}{2 \mu_{0}}\right)+\frac{1}{\mu_{0}}(\boldsymbol{B} \cdot \nabla) \boldsymbol{B},
$$

where the term $B^{2} /\left(2 \mu_{0}\right)$ is generally defined as the magnetic pressure $p_{\mathrm{M}}$. The substitution of Eq. 2.16 into Eq. 2.13 will eliminate the current and form the momentum equation of Magnetohydrodynamics:

$$
\varrho\left[\frac{\partial \boldsymbol{v}}{\partial t}+(\boldsymbol{v} \cdot \nabla) \boldsymbol{v}\right]=-\nabla p-\nabla\left(\frac{B^{2}}{2 \mu_{0}}\right)+\frac{1}{\mu_{0}}(\boldsymbol{B} \cdot \nabla) \boldsymbol{B}
$$

\section{Induction Equation}

The induction equation relates the velocity of an electrically conductive fluid to the magnetic field. In particular, this equation describes the phenomenon of a magnetic dynamo. The substitution of the electric field $\boldsymbol{E}$ in Faraday's law (Eq. 2.8) yields:

$$
\frac{\partial \boldsymbol{B}}{\partial t}=-\nabla \times \boldsymbol{E}
$$

and with the help of the generalised Ohm's Law

$$
\boldsymbol{j}=\sigma(\boldsymbol{E}+\boldsymbol{v} \times \boldsymbol{B}),
$$

this leads to the induction equation of Magnetohydrodynamics:

$$
\frac{\partial B}{\partial t}=\nabla \times(\boldsymbol{v} \times \boldsymbol{B})-\frac{1}{\sigma \mu_{0}} \nabla \times(\nabla \times \boldsymbol{B}) .
$$

Here, the electrical conductivity $\sigma$ is regarded as constant. The model describes how a magnetised plasma responds to fluid motion and vice versa. The ratio of the two terms reveals which one dominates over the other. In a similar fashion to the fluid equations, one can define the dimensionless Magnetic Reynolds Number $R_{M}$ :

$$
R_{M}=\frac{\nabla \times(\boldsymbol{v} \times \boldsymbol{B})}{\eta \nabla^{2} \boldsymbol{B}} .
$$

In order to arrive at Eq. (2.21), the vector identity from Eq. (2.15), the solenoid constraint $\nabla \boldsymbol{B}=0$ and the definition of the magnetic diffusion $\eta=\left(\sigma \mu_{0}\right)^{-1}$ were used. For high Reynolds numbers realised via a perfect conductive fluid $\left(R_{M} \rightarrow \infty, \sigma \rightarrow \infty\right)$, drag effects can be neglected, which will reduce the induction equation to the following form:

$$
\frac{\partial \boldsymbol{B}}{t}=\nabla \times(\boldsymbol{v} \times \boldsymbol{B}) .
$$




\section{Ideal Magnetohydrodynamic Equations}

Thus, the closed set of Magnetohydrodynamic equations is:

$$
\begin{array}{cc}
\frac{\partial \varrho}{\partial t}+\nabla(\varrho \boldsymbol{v})=0 & \text { (Mass Continuity Eq.) } \\
\frac{d}{d t}\left(\frac{p}{\varrho^{\gamma}}\right)=0 & (\text { State Eq.) } \\
\varrho \frac{d \boldsymbol{v}}{d t}=-\nabla p-\frac{1}{\mu_{0}} \boldsymbol{B} \times(\nabla \times \boldsymbol{B}) & (\text { Euler Eq.) } \\
\frac{\partial \boldsymbol{B}}{\partial t}=\nabla \times(\boldsymbol{v} \times \boldsymbol{B}) . & (\text { Induction Eq.) }
\end{array}
$$

All dissipative processes resulting from thermal conductivity, electrical resistivity or finite viscosity are not considered in this formulation.

\subsubsection{Application of Magnetohydrodynamics to the Solar Corona}

The magnetic field $B$ constraints particles in the coronal plasma to perform spiral gyromotions along magnetic field lines. If the kinetic energy of a particle exceeds the magnetic field energy, it can escape from its gyroorbit. This behaviour can be described by the plasma- $\beta$ parameter which is defined as the ratio of thermal against magnetic pressure in a plasma

$$
\beta=\frac{p_{t h}}{p_{m a g}}=\frac{n k_{\mathrm{b}} T}{B^{2} / 2 \mu_{0}}
$$

with the temperature $T$, the particle number density $n$, the Boltzmann constant $k_{\mathrm{b}}$, the magnetic flux $B$ and the magnetic constant $\mu$. Table 2.1 gives an overview of the physical properties from the photosphere to the outer corona. For $\beta<1$, the structure of a plasma is dominated by the magnetic field. For $\beta>1$, the magnetic field is frozen to the plasma and tied to its motion. As illustrated in Figure (2.18), magnetic forces are controlling the structure formation in the upper chromosphere and the lower corona, creating structures such as filaments, coronal loops corona and helmet streamers. In the outer corona, the

\begin{tabular}{|c|c|c|c|c|}
\hline parameters & photosphere & cool corona & hot corona & outer corona \\
\hline electron density $n_{e}\left(\mathrm{~cm}^{-3}\right)$ & $2 \times 10^{17}$ & $1 \times 10^{9}$ & $1 \times 10^{9}$ & $2 \times 10^{7}$ \\
\hline temperature $T(\mathrm{~K})$ & $5 \times 10^{3}$ & $1 \times 10^{6}$ & $3 \times 10^{6}$ & $1 \times 10^{6}$ \\
\hline pressure $p\left(\right.$ dyne $\left.\mathrm{cm}^{-2}\right)$ & $1.4 \times 10^{5}$ & 0.3 & 0.9 & 0.02 \\
\hline magnetic field $B(\mathrm{G})$ & 500 & 10 & 10 & 0.1 \\
\hline plasma- $\beta$ & 14 & 0.07 & 0.2 & 7 \\
\hline
\end{tabular}
plasma- $\beta$ increases, which forces the magnetic field to follow the motion of the plasma. This leads to the effect that the interplanetary magnetic field winds up to the form of a spiral (compare with Section 3.2).

Table 2.1: Plasma parameters in the photosphere and the corona. (Aschwanden, 2004) 


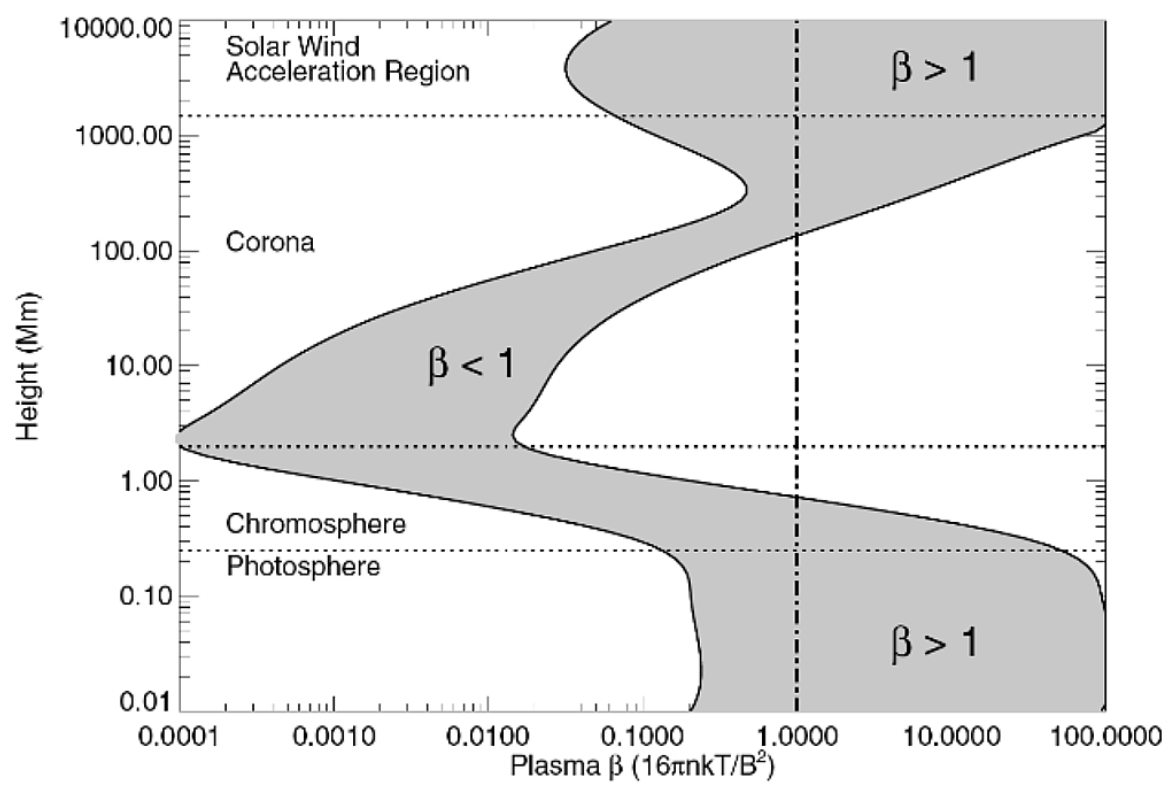

Fig. 2.18: Typical plasma- $\beta$ range for the different layers of the solar atmosphere for a magnetic field of the strength 100-2500 G. (Gary, 2001) 


\subsection{Coronal Large-Scale Structures}

The distribution of the coronal plasma is strongly connected to the dynamics and topology of the solar magnetic field. Therefore, the corona is characterised by an abundance of large scale structures. In Figure (2.19) the large scale structures of the outer corona are related to different phenomena of the inner corona. Two major representatives of these phenomena are helmet streamers and coronal loops.
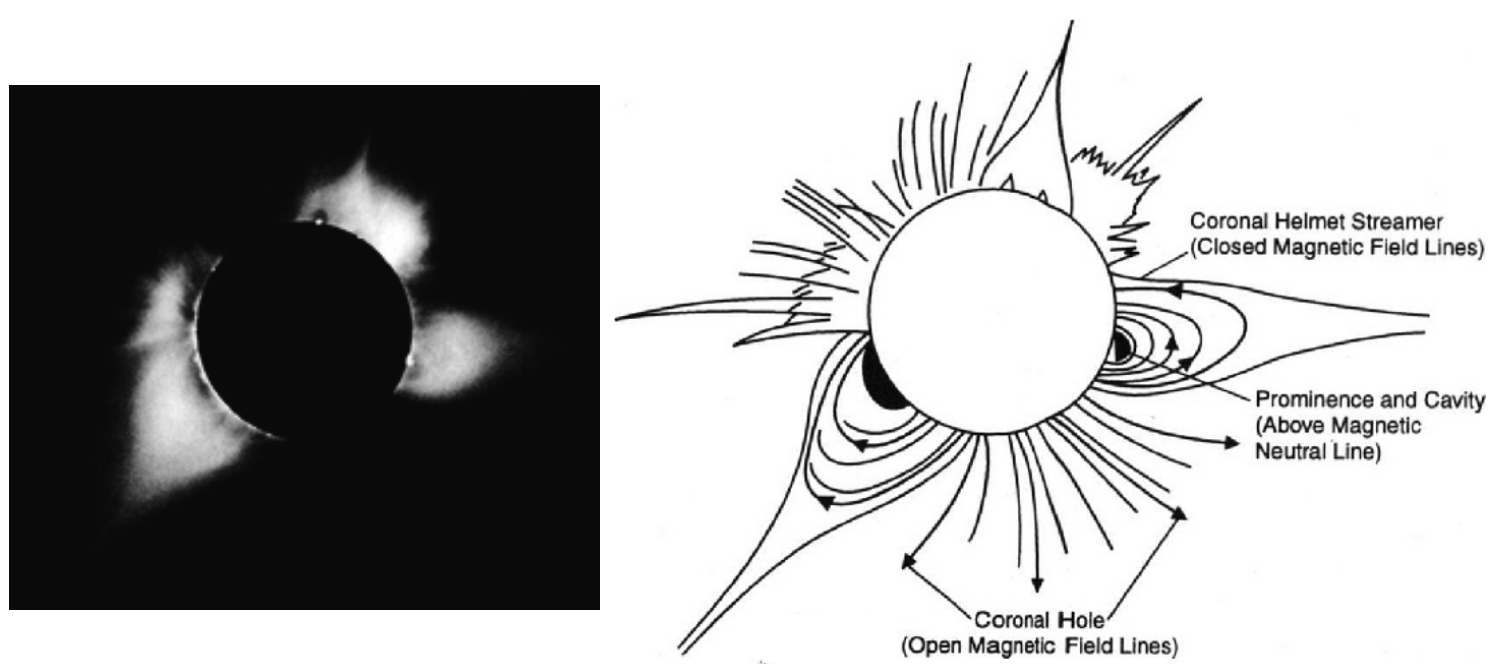

Fig. 2.19: Left: Image of the solar corona during the solar eclipse on 12 November 1966, Peru. Right: Sketch of the associated coronal structure and respective magnetic field line configuration. (Russell 2001, adapted from Hundhausen 1995).

Helmet Streamers are formed in the inner corona by the interaction of the solar wind with the solar magnetic field (Mikić \& Linker, 1996). Often, these structures extend above prominences, far into the corona. They manifest as bright cap-like structures with long pointed peaks, which are usually foot-pointed over active regions and sunspots, connecting regions of opposite magnetic polarity. During solar minimum, helmet streamers are confined to the "streamer belt" close to the equator. However, they follow the movement of active regions during the solar cycle. The second phenomenon, Coronal Loops, are highly twisted and closed magnetic field lines which are anchored in the photosphere (see Figure 2.20) at regions of strong opposite magnetic polarity. They can stretch out into the corona up to hundreds of thousands of kilometres. They function as "highways" for photospheric material which exits with coronal temperatures and propagates along these lines. Coronal loops appear in many different scales and depending on their temperature, which scatters widely around $1 \mathrm{MK}$, they radiate in different wavelengths. The population of coronal loops is connected to the sunspot cycle and the grade of solar activity. Bright coronal loops are more common around the solar maximum, whereas fainter ones, lasting up to weeks, are mostly observed during solar minimum. The connection between largescale structures and the inner corona is well recognisable in the observations taken on July 11, 2011 around a solar eclipse in Figure (2.21). 


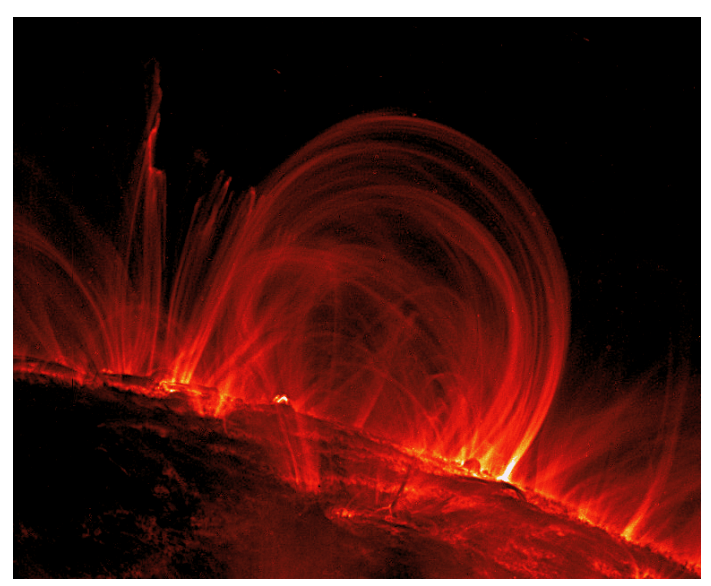

Fig. 2.20: Coronal loops in the solar atmosphere observed with TRACE in $171 \AA$ on November 6, 1999 at 2:30 UT. (NASA/TRACE)

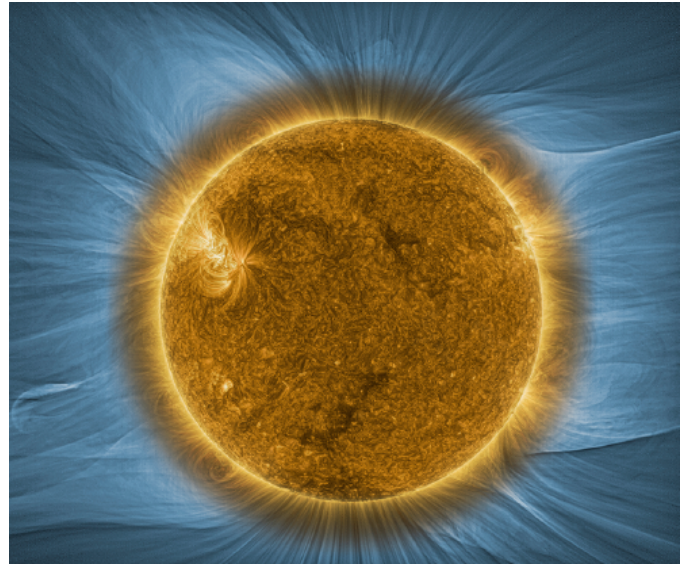

Fig. 2.21: Composite image of the whitelight eclipse observation (blue) and the corresponding inner corona (yellow) taken with SDO/AIA in $171 \AA$ on July 11, 2010. (Karen Teramura)

\subsection{The Magnetic Sun}

\subsubsection{The Solar Dynamo}

The Solar Dynamo is a physical model describing the self sustaining generation of the solar magnetic field. In this model, the kinetic energy associated with convection underneath the solar surface is converted into electromagnetic energy. The so created solar magnetic field plays a key role in the dynamics and topology of all phenomena in the solar corona. During a full solar cycle, the initial poloidal structure of the solar magnetic field evolves due to the differential rotation of the Sun. The frozen-in magnetic field lines manifest in a steadily growing toroidal magnetic field component (see Figure 2.22), a process which is known as the $\omega$-Effect. By a second mechanism, termed $\alpha$-Effect, buoyancy causes the rise of magnetic flux ropes which twist under the simultaneously acting Coriolis force. The rising field tubes pierce the solar surface at two spots with opposite magnetic polarities (Parker, 1955; Babcock, 1961). The global twisting of the magnetic field causes the field to periodically flip its polarity in an 11-year cycle. A solar cycle starting with a magnetic North-South configuration will pass a phase with complex toroidal components and finally result in a South-North configuration at the start of the next cycle. An overview of current solar dynamo models can be found in Charbonneau (2010).

\subsubsection{Active Regions}

Active regions (ARs) are areas of highly concentrated magnetic field lines, extending from the photosphere to the corona (see Fig 2.23). They are revealed by a wide spectral emission from radio to X-ray and, during solar flares, even in the $\gamma$-ray spectrum. Interestingly, they seem to produce $82 \%$ of the total coronal heating energy (Aschwanden, 2001). ARs are formed by the emergence of photospherical, strong magnetic flux and hence, in their simplest form, have a bipolar magnetic field configuration (see Figure 2.24). The strong magnetic fields of ARs manifest in the photosphere as dark sunspots. In the chromosphere, filaments form at their magnetic inversion line. In the transition region and corona bright, hot and dense (coronal) loops connect opposite magnetic polarities. ARs are the birthplace of most solar activity, ranging from small-scale brightenings to large solar flares and coronal 


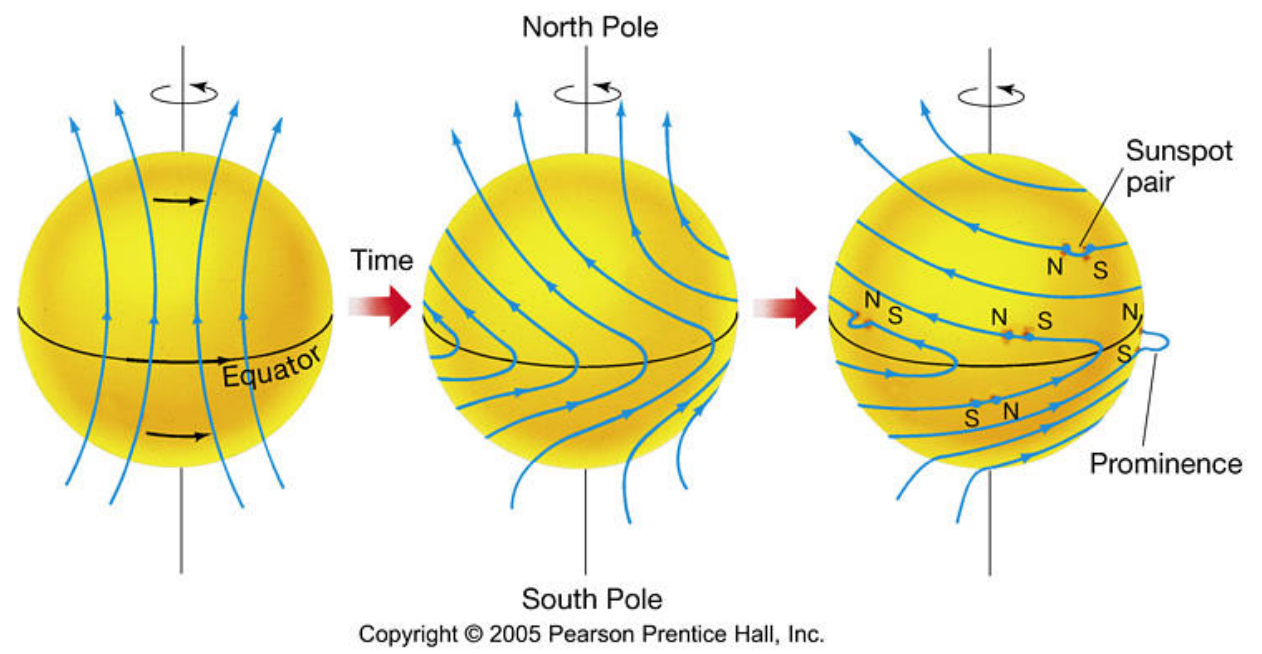

Fig. 2.22: Differential rotation of the Sun causes the frozen poloidal magnetic field to be dragged around in toroidal direction resulting in an azimuthal magnetic field. Buoyancy forces cause the rise of magnetic flux ropes. The piercing of the surface by flux ropes generates a sunspot pair with opposite magnetic polarity. Sunspot pairs in the same hemisphere have always the same otherwise opposing polarity configuration. In this figure the sun spots of the northern hemisphere have a North-South configuration. With each polarity switch of the global solar magnetic field, this configuration gets inverted as well.

mass ejections (van Driel-Gesztelyi \& Green, 2015). It was recognised that the leading spot of a bipolar active region is positioned closer to the equator on both hemispheres. Today this is known as Joy's Law (Hale et al., 1919). Furthermore, the formulation of Hale's Law (Hale, 1924) points out that the polarity order of active regions depends on the hemisphere in which the pair is positioned. It is of opposite order across the equator, reversing after each sunspot cycle. These observations are constraining solar dynamo models and therefore implying that ARs are the result from the emergence of $\Omega$-loops rooted in the tachocline region (Charbonneau, 2010).

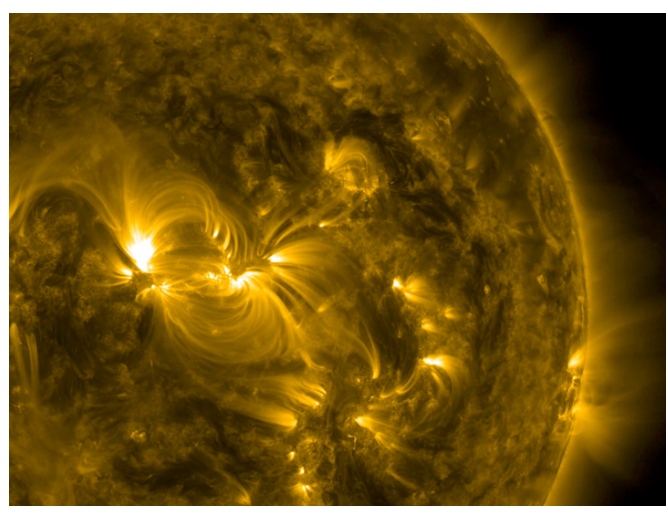

Fig. 2.23: Active region observed with NASA's Solar Dynamics Observatory in $171 \AA$ wavelength of extreme ultraviolet light on 15 July 2016. Hot plasma spirals along the closed magnetic field lines and forms coronal loops. (NASA - David Hathaway)

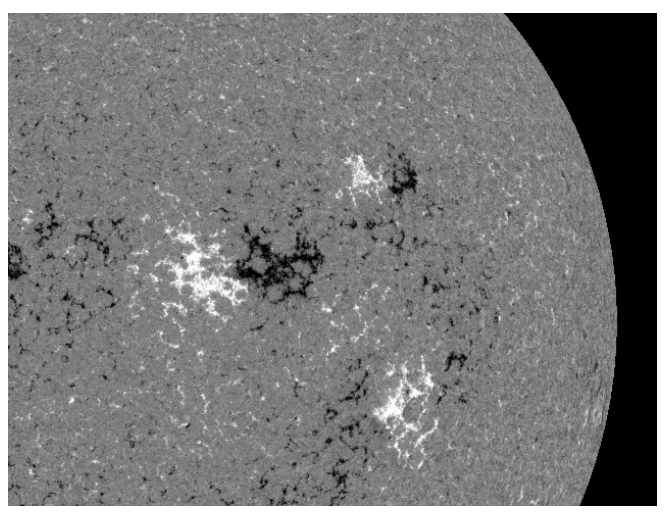

Fig. 2.24: Same active region observed with the SOHO/Helioseismic and Magnetic Imager (HMI) (Scherrer et al., 2012), showing photospheric magnetic fields of opposite polarity as light and dark regions. (NASA) 


\subsubsection{Sunspots}

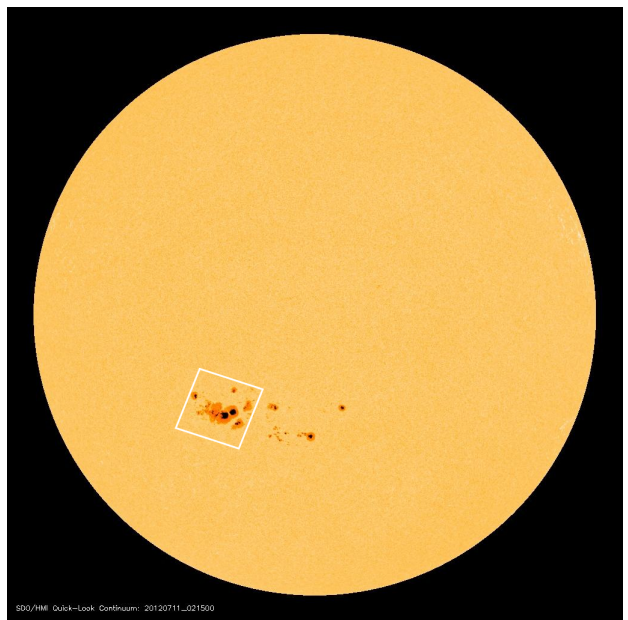

Fig. 2.25: Chromosphere on July 11, 2012 observed by SDO with the Helioseismic and Magnetic Imager (HMIContinuum). A large sunspot group extends over $300,000 \mathrm{~km}$. (NASA)

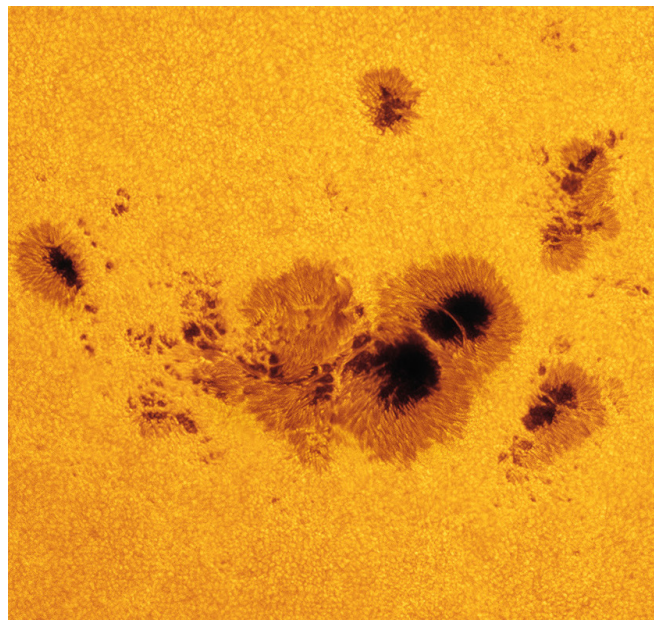

Fig. 2.26: Close up view of a sunspot group. At the centre, a big sunspot with a dark umbral core is surrounded by penumbral filaments. (Alan Friedman)

Sunspots have typical sizes of 3.5-60 Mm and appear in the visible light spectrum as dark regions on the solar surface (Figure 2.25 and 2.26). They can be considered as photospheric manifestation of active regions. Strong torodial magnetic flux tubes with strengths of up to $100 \mathrm{kGs}$ emerge from the photosphere into the solar corona. Sunspots have a dark appearance because of their considerably lower temperature $(\sim 3,800 \mathrm{~K})$ compared to the surrounding photosphere $(\sim 5,800 \mathrm{~K})$. They can often be observed in groups and generally persist between a few days and a few months. Historically, many insights in the nature of the Sun were gained from observations of sunspots. The first sunspot observations were made by different cultures with the naked eye over 2000 years ago (Bray \& Loughhead, 1964). In the year 1611 David and Johannes Fabricius concluded from the movement of sunspots around the solar disk that the Sun is in fact rotating - an idea which was already suggest by Giordano Bruno and Johannes Kepler. The awareness that this rotation has a differential character resulted from the fact that sunspots rotate faster at lower latitudes. Hale (1908b) discovered the Zeeman splitting of spectral lines in sunspots. Therefrom we know that the lower temperatures of sunspots are a result of overlying magnetic loops. These loops, rooted with their footpoints in the photosphere, inhibit the convective motion of the plasma in this area. From a powerful magnetic field concentration, a complex active region can develop. Similar to the bipolar ARs, sunspots are usually seen in pairs of different polarities suggesting that magnetic field lines emerge from one spot and re-enter at the conjugated one.

\subsubsection{The Solar Cycle}

The solar cycle describes the periodically changing global magnetic activity of the Sun, which can be quantified by the monthly sunspot count. Long term observations revealed an 11-year sunspot periodicity, which was discovered in 1843 by Samuel Heinrich Schwabe. In fact, the period is not strictly constant but varies between $\sim 9$ and 11.5 years. The annual activity change of the Sun is presented in Figure 2.27 by an annual snapshot in the 
ultraviolet spectrum. The solar cycle is defined from one activity minimum to the next

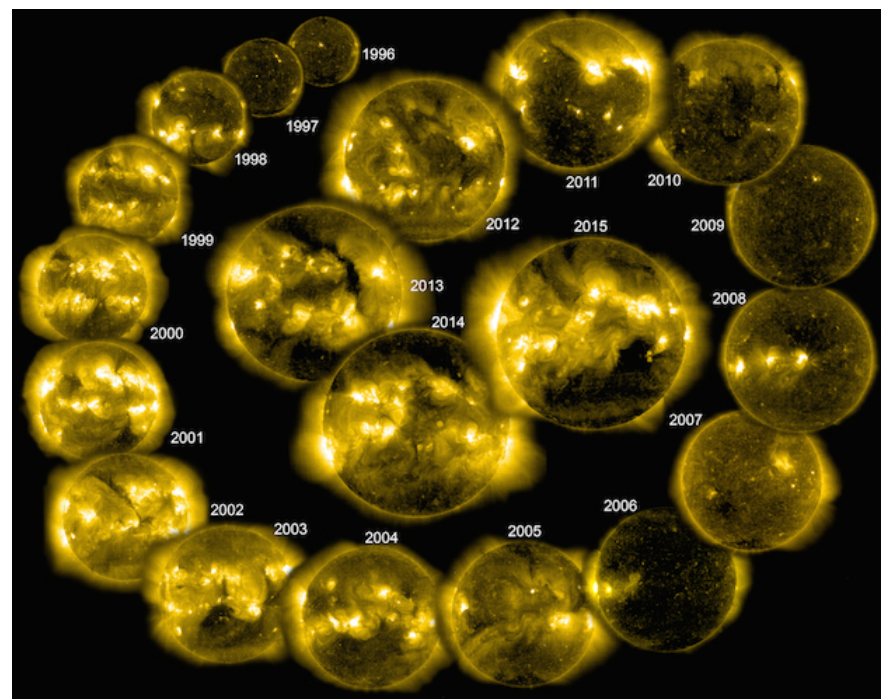

Fig. 2.27: Collage of 20 annual snapshots of solar activity with SOHO/EIT in $284 \AA$. (ESA\&NASA)

and numbered progressively after the first defined solar cycle between the years 1755-1766. In the same way that the seasons determine the boundary conditions of our climate on Earth, the solar cycle determines the boundary conditions for the solar activity, affecting all kinds of solar phenomena, including photospheric features, prominences, streamers and CMEs. Even the form and shape of the corona, the solar wind, the heliosphere and the interplanetary magnetic field are defined by the solar cycle. On longer timescales the solar cycle (see Figure 2.28) shows periods of almost no solar activity during the Maunder Minimum and phases of increased activity, like the current Modern Maximum. These observation are consistent with the discovery of an anomalous abundance of $\mathrm{C}^{14}$ assimilated in trees (Usoskin, 2017). Such an idiosyncrasy of the solar cycle can be described within the field of non-linear dynamics. In this case the solar cycle would be considered a strange attractor with a quasi-periodic modulation (Ruzmaikin, 1981). The occurrence and the role of the 70 years lasting Maunder Minimum, starting in 1650, is still controversially discussed. Some researchers are convinced that the Maunder Minimum was the cause of the Little Ice Age, but a recent study by Feulner \& Rahmstorf (2010) showed that the average global temperature has not decreased more than a third of a degree due to this extreme low solar activity phase.

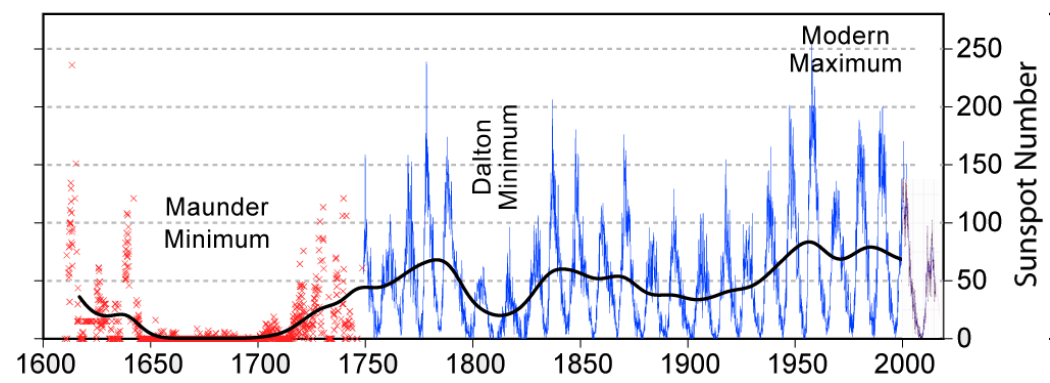

Fig. 2.28: Four hundred years of sunspot observations reveal considerable fluctuations of maximal solar activity. (Image prepared by Robert A. Rhode as part of the Global Warming Art project) 
The Butterfly Diagram is another visualisation of the periodic fluctuation of the sunspot cycle. In this diagram, the latitudinal sunspot position is plotted over time (Figure 2.29). During a sunspot cycle the maximal latitude for the occurrence of sunspots migrates from higher absolute latitudes $\left(35^{\circ}\right)$ to lower ones $\left(5^{\circ}\right)$. This was first noted by Carrington (1858) and after the refinement of this observation by the German astronomer Gustav Spörer, the phenomenon became known as Spörer's Law. The migrating behaviour of the sunspots originates from the transition of the Sun's initial poloidal dipolar field towards higher toroidal magnetic field components. Thereupon Babcock (1961) developed a qualitative model of the solar magnetic field with 11-year cycle polarity change, claiming a true solar cycle periodicity of 22-years after which the original magnetic configuration is restored, also often referred to as the 22-year Babcock-Leighton solar dynamo cycle.
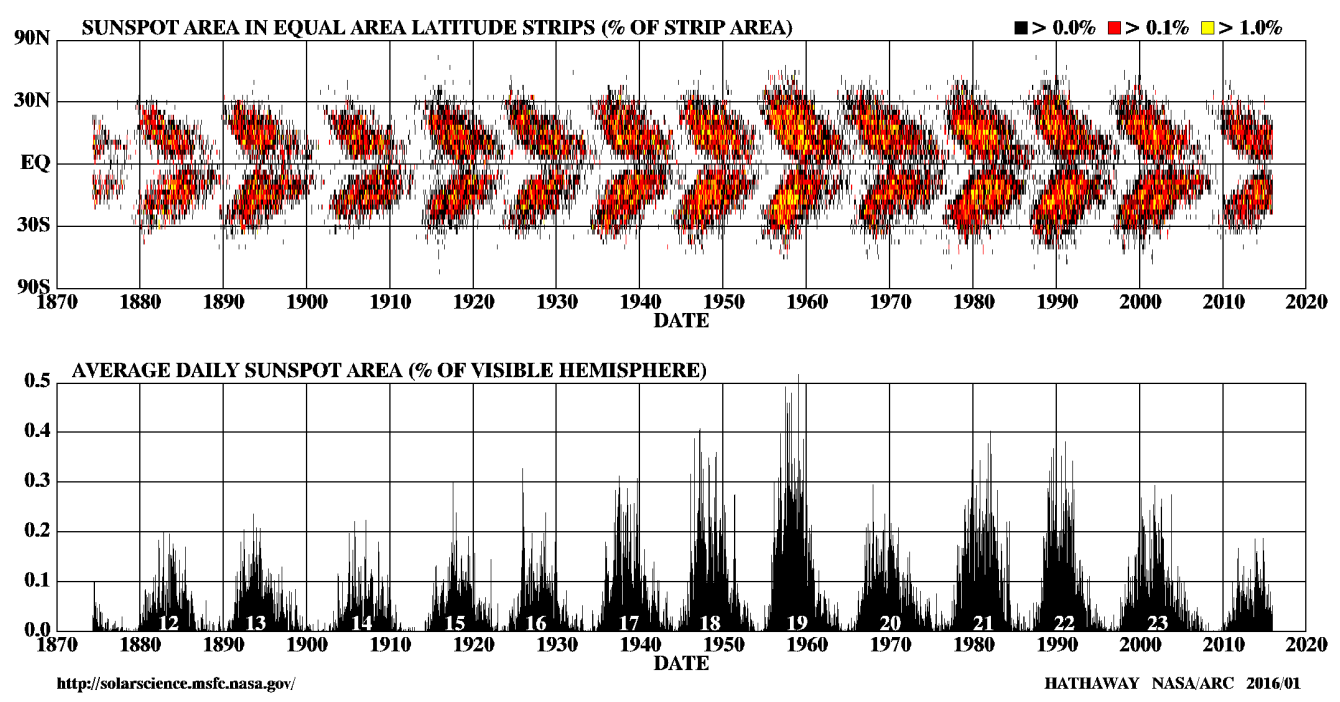

Fig. 2.29: Daily Sunspot area averaged over individual solar rotations. Top: Sunspot observations of the Royal Greenwich Observatory. Sunspots are concentrated on two latitude bands on each side of the equator (after E.W. Maunder) with decreasing absolute maximum latitudes towards the end of each solar cycle. Bottom: The 11-year sunspot cycle. The number of sunspots is a proxy for the solar magnetic activity. (NASA - David Hathaway) 



\section{Interplanetary Space}

It is a common misconception the interplanetary space consists of a perfect vacuum. In fact, this space is filled with a continuous outflow from the upper atmosphere of the Sun the solar wind, which contains dust particles of low density, magnetic fields and plasma. CME dynamics are highly affected by the interaction with the solar wind during their heliospheric propagation. This chapter gives a brief overview about not only the solar wind and the interplanetary magnetic field but also about their roles in creating the heliosphere and shaping Earth's magnetosphere.

\subsection{Solar Wind}

The solar wind is a steady, supersonic outflow of coronal plasma and magnetic field from the Sun. It permeates the interplanetary space and is driven by the heliospheric pressure gradient. Two types of solar wind are known:

- The slow solar wind has a speed of less than $450 \mathrm{~km} / \mathrm{s}$, particle number densities between 7 and $10 \mathrm{~cm}^{-3}$, temperatures of around $4 \times 10^{4} \mathrm{~K}$ and a particle composition that is more similar to the corona.

- By contrast, the fast solar wind is swifter $(450-800 \mathrm{~km} / \mathrm{s})$, less dens $\left(3 \mathrm{~cm}^{-3}\right)$, hotter $\left(2 \times 10^{5} \mathrm{~K}\right)$ and the particle composition is a closer match to the solar photosphere.

These different characteristics of both solar wind types can be explained by their different origins. During solar minimum, the slow solar wind arises from the heliospheric current sheet near the equator and in solar maximum from the tips of helmet streamers. The fast solar wind commonly origins from coronal holes. The discovery of the solar wind goes back to the German astronomer Ludwig Biermann (1951). It is closely connected to comets which can be regarded as natural probes of the heliosphere. Comets, like the Hale-Bob comet (see Figure 3.2), can posses two fundamentally different types of tails. The dust tail is curved and directed away from the Sun due to the solar radiation pressure. In contrast, the ionised gas tail is not curved. Biermann explained this phenomenon by postulating an additional corpuscular charged radiation acting on the gas tail, today known as solar wind. It was noticed that the solar wind shows a recurrent behaviour with the synodic solar rotation period of 27 days. Further, the solar rotation leads to a toroidal spiraling of the radial outward flowing solar wind. This configuration is known as Parker Spiral (Parker, 1958). It impacts Earth, at $1 \mathrm{AU}$ distance, with an angle of $\sim 45^{\circ}$. As the spiraling effect for the fast solar wind is less intense than for the slow one, both will inevitably collide and form Co-rotating Interaction Regions (CIRs) (see Fig 3.1). Such CIRs are characterised by high densities and have a chance to also form shock waves. 


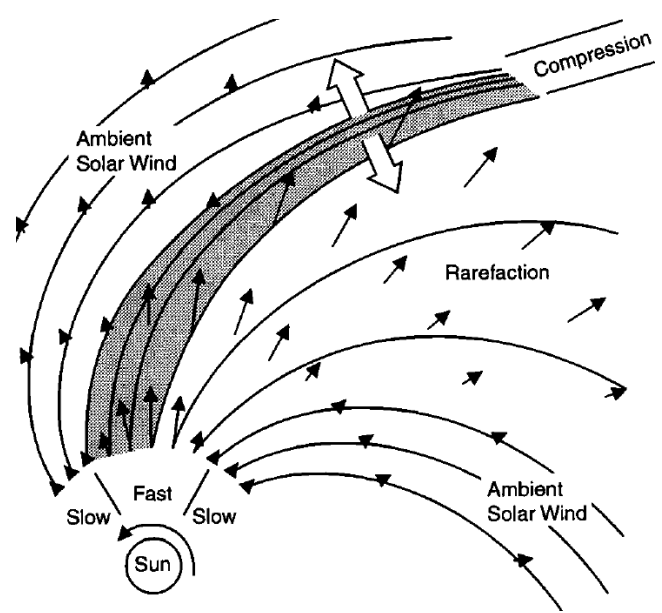

Fig. 3.1: Scheme of the interaction of fast and slow solar wind streams leading to the formation of highly compressed co-rotating interaction regions. (Russell 2001, adapted from Pizzo 1985.)

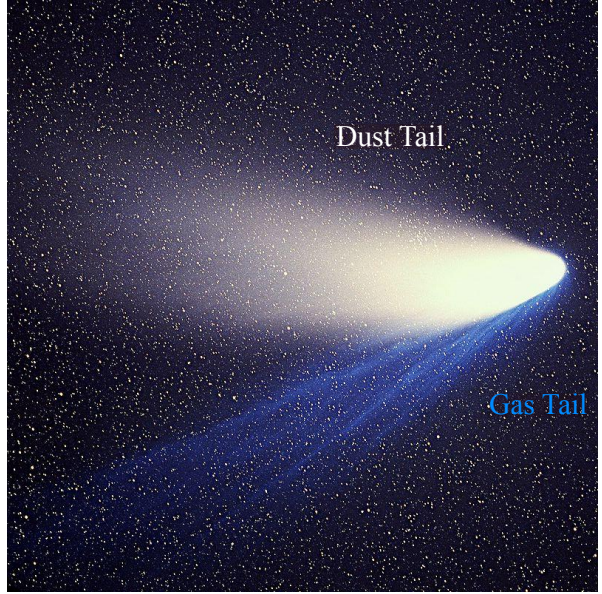

Fig. 3.2: Image of Comet Hale-Bopp taken on 1997 April 4 with a blue, ionised gas tail and a white dust tail. (Image: E. Kolmhofer, H. Raab - Johannes-Kepler-Observatory, Linz, Austria)

\subsection{The Interplanetary Magnetic Field}

The interplanetary magnetic field (IMF) is created by solar magnetic field lines, emerging radially outward from the solar surface. The term IMF is often used in a more narrow sense to reference the ecliptic component only. At the beginning of the solar cycle, the solar magnetic field has a pure dipole configuration. A simulation of the solar magnetic field during the solar minimum is shown in a longitudinal cut in Figure 3.3. The magnetic field

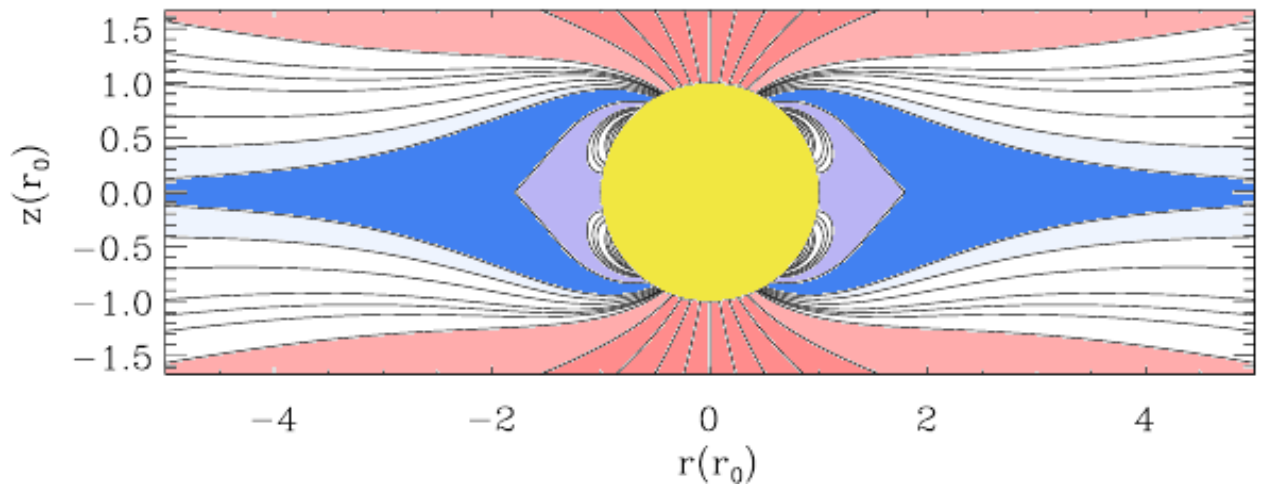

Fig. 3.3: Coronal magnetic field for a dipole configuration with additional quadrupole components in the solar minimum. Adapted from the Dipole plus Quadrupole plus Current Sheet model (DQCS) by Banaszkiewicz et al. (1998).

strength of a straight dipole field decreases according to $B(R) \sim R^{2}$ with the distance $R$. Surprisingly, the real field strengths of 1-10 nT measured at Earth, exceeds this expectation by a hundredfold due to the electrically conductive plasma from the solar wind. At heights above $4-20 R_{\odot}$, the IMF becomes bound to the solar wind (compare with Figure 2.18) and is therefore carried out much further. The binding to the plasma motion and the solar rotation also causes the IMF to wind up into the form of an Archimedian spiral. A special phenomenon of the IMF is the Heliospheric Current Sheet (HCS), the biggest 


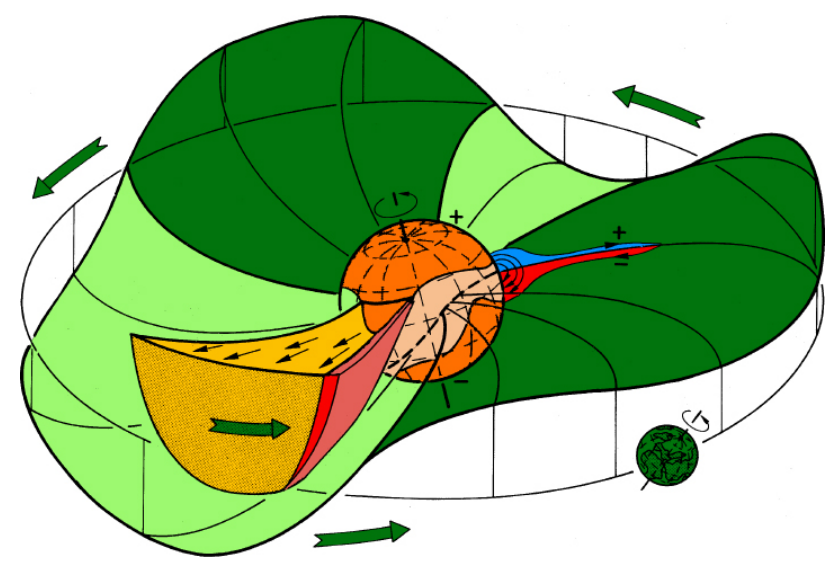

Fig. 3.4: Ballerina Skirt Model of the 3-D Heliospheric Current Sheet proposed by Alfven (1977). (Schwenn, 2006)

structure in the heliosphere. In a sheet around the Sun, the solar magnetic field changes polarities resulting in a small electrical current with a radial component. The HCS has a thickness of around $10.000 \mathrm{~km}$ at $1 \mathrm{AU}$ and a maximum current density in the order of $10^{-10} \mathrm{~A} / \mathrm{m}^{2}$. As the magnetic solar axis is tilted by $7.5^{\circ}$ towards Earth, HCS can be described with the Ballerina Skirt Model (Alfven 1977; Figure 3.4). When the spinning skirt passes Earth, a magnetic sector boundary is crossed and a polarity switch can be measured. The latitudinal interplanetary magnetic field was investigated with the Ulysess spacecraft launched in the 1990. The measurements showed that the solar magnetic field at the poles is much stronger than near the equatorial plane (Figure 3.5). Also the clear global dipole configuration around the solar minimum develops towards a chaotic configuration around the solar maximum, which is directly associated with the different appearance of the solar corona during the solar cycle (compare with Figure 2.15).

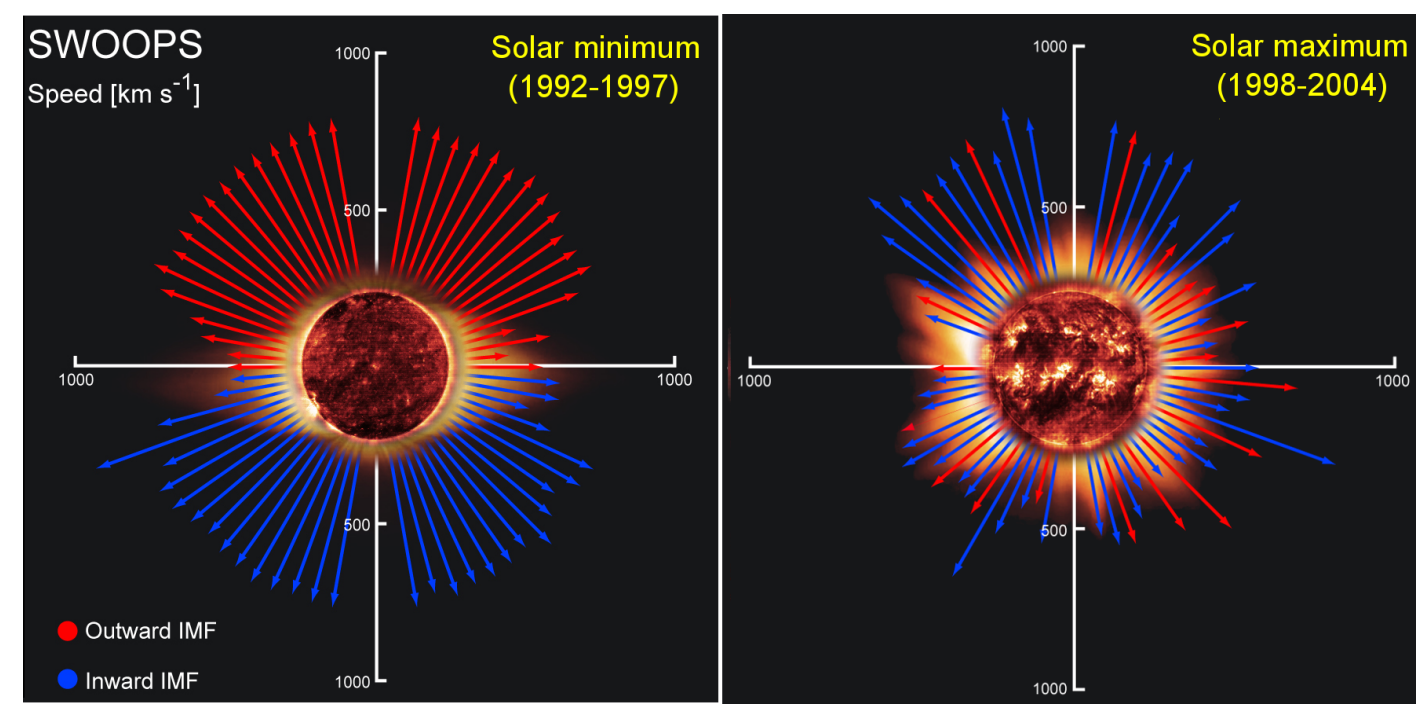

Fig. 3.5: Measurement of the latitudinal solar wind with the SWOOPS (Solar Wind Observations Over the Poles of the Sun) instrument of the Ulysses space probe (Wenzel \& Smith, 1992) in the time frame of the solar maximum (top) and solar minimum (bottom). (ESA - NASA) 


\subsection{The Magnetosphere}

Before space weather related phenomena can affect Earth they are firstly confronted with the "magnetosphere", a term coined by T.Gold in 1959. The magnetosphere provides Earth with a natural shielding against electromagnetic impacts from the solar and interstellar medium (see Figure 3.6). This Earth-surrounding bubble, consists of charged particles which are controlled by the Earth's dipole magnetic field. The magnetosphere is significantly distorted by the flow of solar wind. On the dayside of Earth, the magnetosphere is compressed to a distance of about $65,000 \mathrm{~km}$.

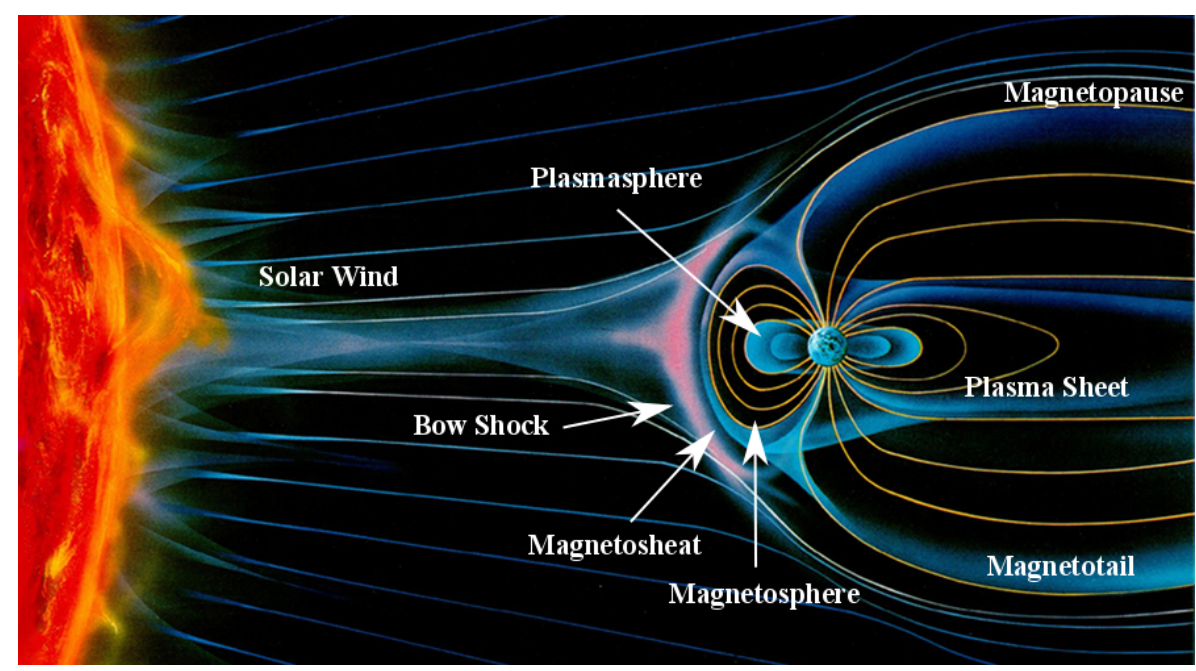

Fig. 3.6: Schematic illustration of the magnetosphere. (Image: Yoshuke Kamide - National Geophysical Data Center)

The bow shock is a supersonic shock wave located around 90,000 km away from Earth. It can be considered as a boundary between the magnetosphere and the ambient medium. The magnetosheath is the region behind the bow shock and serves as a "cushion" between the solar wind flow and the magnetic field barrier. In this region, solar wind particles, which are decelerated and heated at the bow shock, detoured around Earth. In the magnetopause the pressure of Earth's magnetic field and the pressure of the solar wind are in an equilibrium state. The magnetopause changes size and forms as the pressure of the solar wind fluctuates. Large amounts of plasma penetrate the magnetopause provoking magnetic reconnection and enabling particles to enter the magnetosphere. On the night side, the magnetic field forms the magnetotail, which is stretched to a length of 6.3 million kilometers. Most of the volume in the magnetotail is taken up by the northern and southern tail lobs which are separated by the plasma sheet, a region with dense hot plasma and lower magnetic field separating the two tail lobes. In the direction away from the Sun the field lines of the tail lobes connect to the solar wind. The plasmasphere, or inner magnetosphere, is a torus shaped region consisting of low energy plasma. The magnetic field of the plasmasphere captures high-energy charged particles which then drift around the Earth in the Van Allen Belt. The connection between CMEs and geomagnetic storms and CMEs was made by Wilson (1987), showing that a strong southward component of the magnetic field in magnetic clouds leads to a large geomagnetic disturbance. 


\subsection{The Heliosphere}

The Sun is embedded in the local cloud of interstellar matter. This cloud is a result from a supernova explosion a few million years ago and consists of neutral and ionised atoms, as well as dust of various grain sizes. As the Sun moves through the cloud, it continuously emits the solar wind. The interaction of the solar wind with the counterdirected interstellar medium, which has a speed of about $100 \mathrm{~km} / \mathrm{s}$, forms the heliosphere (see Fig 3.7), a bubble-like cavity, which extends far beyond Pluto. The solar wind does

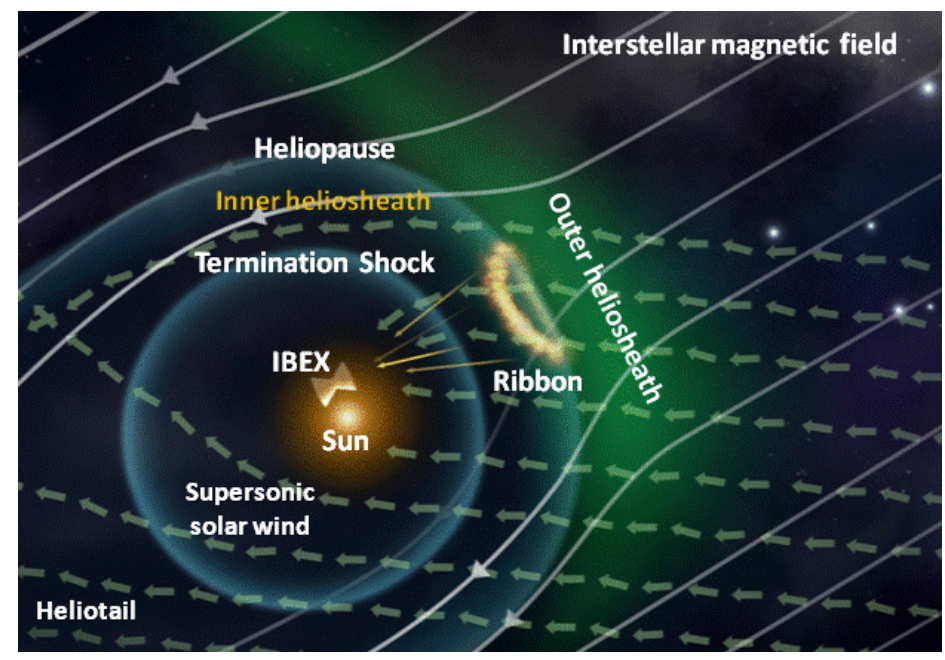

Fig. 3.7: Schematic of the heliosphere in the interstellar medium. From the Laboratory for Solar System Physics \& Astrophysics, Space Research Centre of the Polish Academy of Sciences. Graphics design: Marzena A. Kubiak, Maciej Frołow, Tentaris.

not extend infinitely. At around 75 to $90 \mathrm{AU}$, it is slowed down to subsonic speed by the pressure of the local interstellar medium, leading to the formation of the termination shock. This region was crossed by Voyager 1 in 2004 and Voyager 2 in 2007 (Burlaga et al., 2008). Behind the termination shock, the region of the inner heliosheath starts at around 80 to $100 \mathrm{AU}$ at its closest point to the Sun in the solar propagation direction. In this region, the solar wind is further slowed down, compressed and made turbulent due to its interaction with the interstellar medium Opher et al. (2009). The heliopause is the outer limit of the inner heliosheath. Here, the pressure of the solar wind becomes balanced to the pressure of the interstellar magnetic field so that the solar wind particles become indistinguishable. The heliopause is hardly penetrable for charged particles, but transparent for neutral atoms. In front of the heliosphere, a disturbed region called the outer heliosheath is formed, where the flows of interstellar plasma and neutral gas decouple. It was hypothesised that the impact of the interstellar medium at the heliosphere creates a bow shock at about 230 AU. The Interstellar Boundary Explorer (McComas et al., 2009) did not observe such a shock (McComas et al., 2012), which suggests that the velocity of the local interstellar medium relative to the Sun is too slow, but may be sufficient to create a bow wave, though a bow shock might occur around other stars. Surprisingly, IBEX has discovered the "ribbon", an arc-like, almost circular region of enhanced neutral atom emission formed close to the heliopause. 



\section{Coronal Mass Ejections and Associated Phenomena}

In this section the morphology, kinematics, formation, emergence and eruption mechanism of CMEs are highlighted. CMEs most often originate from solar active regions and are frequently associated with solar flares and eruptive prominences. All these phenomena and their mutual relation are presented in this chapter. A special focus is placed on the physical background of CME formation, flux rope eruption and emergence models.

Coronal Mass Ejections (CMEs) are huge outbursts of solar coronal plasma and magnetic fields. An overview of their discovery was already given in introduction of this thesis. Following the definition in Hundhausen et al. (1984), CMEs appear as a change in the coronal structure, manifesting as new and discrete white-light features. They are accelerated beyond the gravitational influence of the Sun and ejected into the heliosphere. The ejected coronal plasma consists of electrons and protons, minor amounts of heavier elements like helium, oxygen, carbon and iron. CMEs occur on average about 0.5 times per day near the solar minimum and about 6 times per day near the solar maximum (Gopalswamy et al., 2003a; Yashiro et al., 2004; Raychaudhuri, 2005; Robbrecht et al., 2009). Unfortunately, CME statistics in the pre-STEREO era suffered from unavoidable projection effects as they are mostly observed with coronagraphs from a single vantage point. Nevertheless, these observations have allowed many statistical studies of CME speed and acceleration. CMEs may reach speeds of up to $2500 \mathrm{~km} / \mathrm{s}$ (Gopalswamy \& Thompson, 2000). The average CME speed is in the order of $480 \mathrm{~km} / \mathrm{s}$ (Webb \& Howard, 2012), where the annual average tends to vary with the solar cycle, with an average $280 \mathrm{~km} / \mathrm{s}$ at solar minimum in 1996 , followed by a steady increase to $520 \mathrm{~km} / \mathrm{s}$ a few years past the solar maximum phase in 2002 (Yashiro et al., 2004).

Illing \& Hundhausen (1985) describe the typical white-light morphology of CMEs as threepart structure consisting of a bright leading loop, followed by a low density cavity (Stenborg et al., 2008) and an embedded bright core. These features are best observable if the CME is directed towards the plane-of-sky of the observer so that it appears as limb event (see Fig. 4.1). Very narrow CMEs with widths below $15^{\circ}$ are often referred to as "jets" and do not contain any aspect of the common three-part structure (Yashiro et al., 2003). The following explanation is therefore referred to wider CMEs, sometimes also termed "normal CMEs" (Gilbert et al., 2001). CMEs, in particular fast ones, exhibit additional leading shock fronts followed by a sheath of post-shock plasma. The outcome is an apparent fivepart structure, composed of a shock front, a diffuse sheath, a bright front, a cavity and a core (Vourlidas et al., 2013). The formation of the shock results from the compression of solar wind material and is further discussed in Section (4.6). The bright CME front consists of hot plasma, which is assumed to result from the mass-pile-up of overlying streamer material (Kahler \& Hundhausen, 1992; Low, 1996). Within a CME structure, the cavity has the lowest plasma density, so that it appears dark in contrast to the front and the core. 


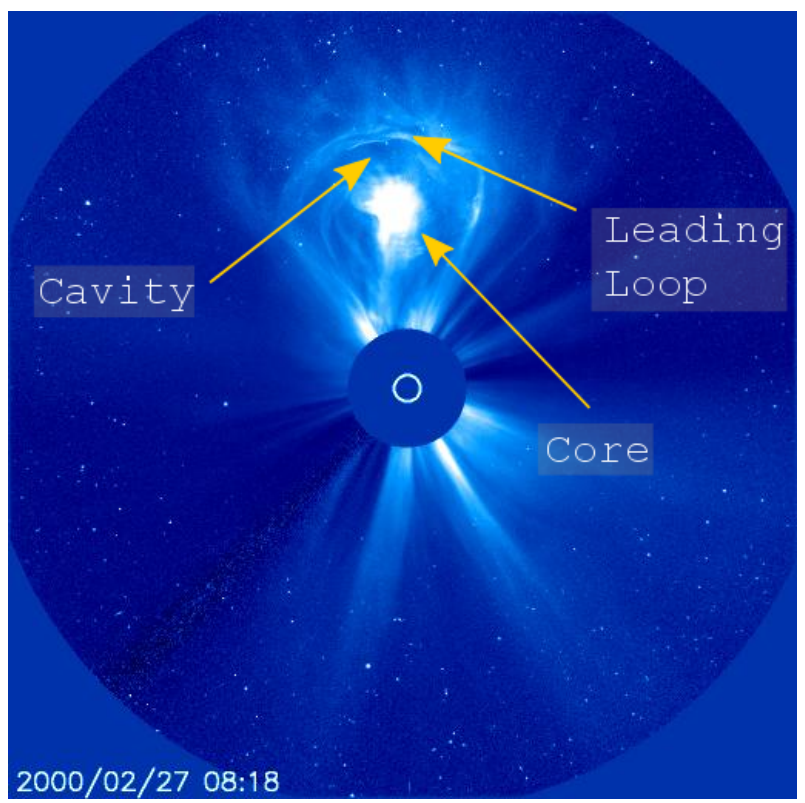

Fig. 4.1: CME three-part structure seen in a CME directed towards the solar limb observed by the LASCO/C3 coronagraph on February 27, 2000. The bright leading loop is followed by a cavity and an even brighter core.

The core is very cold $\left(\sim 4 \times 10^{4} \mathrm{~K}\right)$ and is therefore associated with erupted prominence material originating from the chromosphere. This association is further discussed in Section (4.5). Not all CMEs have cores, as long term observations have proven (Gopalswamy et al., 2001; Yashiro et al., 2003). Often the core has a filamentary structure, but in some cases, especially if the core is very dense, it has a more amorphous appearance. The morphology of CMEs is of special interest to space weather forecasts. Derived from 5 years of Solar Maximum Mission (SMM) observations of over 800 CMEs, Hundhausen (1993) reported that the angular width of CME is on average at $\sim 40^{\circ}$ with a maximum of $\sim 100^{\circ}$. Based on the observation of nearly 7000 CMEs between 1996 and 2002, Yashiro et al. (2004) reported comparable results with increasing angular widths towards the solar cycle maximum but with more extensive maximum widths $\left(\sim 100-360^{\circ}\right)$. The shape variety of CME structures seen in white-light images is quite large. However, most of them result from projection effects (Schwenn, 2006; Cremades \& Bothmer, 2004).

Halo-CMEs, which surround the occulting disk in coronagraph observations, are no exception to this statement. Nevertheless, the article of St. Cyr (2005) differentiates between three definitions of Halo-CMEs. These three definitions are recapitulated in the article "Are Halo coronal mass ejections special events?" by Lara et al. (2006). Firstly, the most common Halo interpretation of Howard et al. (1982) is in fact based on projection effects and describes Halo events as eruptive events near the solar centre directed towards or away of the observer. Thus, Earth-directed CMEs appear as Halos (see Fig. 4.2) or PartialHalos (see Fig. 4.3) in coronagraphs positioned along the Sun-Earth line. Secondly, the Halo-appearance can be the result of a shock wave created by a strong solar eruption. This wave travels through the corona and causes the deflection of streamers and other pre-existing features appearing as a wide perturbation (Hundhausen, 1987; Sheeley et al., 2000a). Lastly, some halo appearances might also result from more than one eruption from the same or an adjacent active region on the solar surface, which can appear as one contiguous structure (St. Cyr, 2005). These active regions are often magnetically connected as shown by statistical investigations (Moon et al., 2002a; Wheatland \& Craig, 2006) as well 


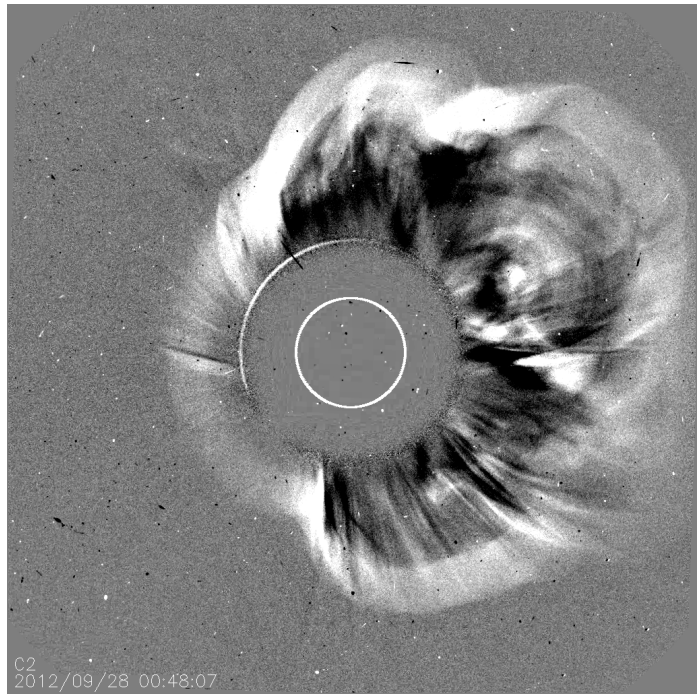

Fig. 4.2: Partial Halo-CME observed by the LASCO/C2 coronograph on September 28, 2012.

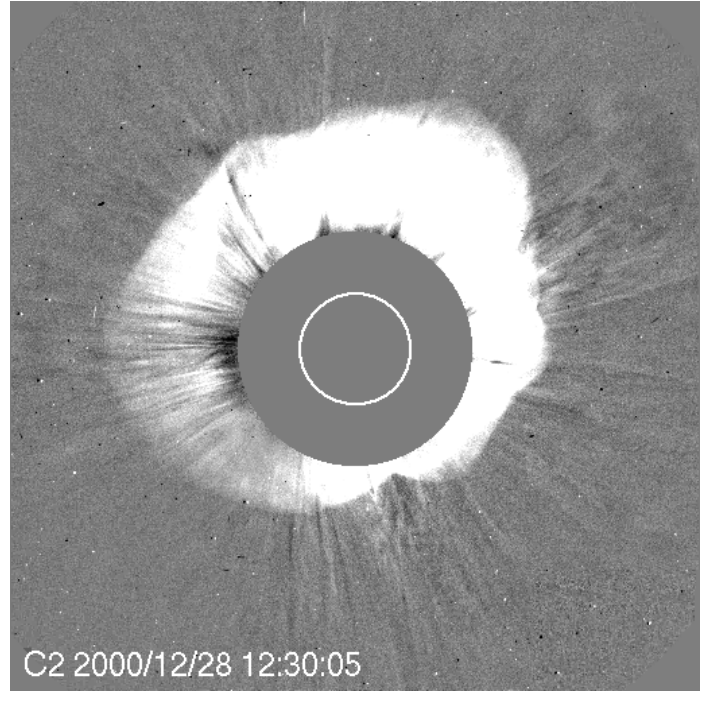

Fig. 4.3: Full Halo-CME observed by the LASCO/C2 coronograph on December 28, 2000 .

as through case studies (Wang et al., 2001), hence they can trigger other active regions to erupt almost simultaneously (Zhukov \& Veselovsky, 2007). Also, a long extended active region is capable of producing more than one eruption (Liu et al., 2009) with only small time shifts in between.

\subsection{Flux Rope Morphology}

Mouschovias \& Poland (1978) were the first to propose bubble-like structures to account for the geometry of CMEs. An early study of electron density distributions of CMEs derived from polarised coronagraph observations also pointed towards this idea (Crifo et al., 1983). Later, long term observations with the LASCO coronagraphs suggested that CMEs might have flux ropes structures. (Chen et al., 2000; Plunkett et al., 2000). Flux ropes are defined as a set of magnetic field lines that wind more than once around a common axial field line. Cremades \& Bothmer (2004) have showed that a cylindrical flux rope geometry (see Figure 4.4) that is elongated along the photospheric neutral line can indeed account for the two-dimensional projection of CMEs in the white-light images of coronagraphs. These results have also been confirmed by Thernisien et al. (2006) through three-dimensional geometrical forward modelling in stereoscopic CME white-light images. Vourlidas et al. (2013) thereupon defined a flux rope CME as an ".. eruption of a coherent magnetic, twist-carrying coronal structure with angular width of at least $40^{\circ}$ and able to reach beyond $10 R_{\odot}$, which occurs on a time scale of a few minutes to several hours."

The study furthermore revealed that of over 2400 events observed with LASCO, at least 40 percent had an unambiguous flux rope structure. Gopalswamy (2013) suggests in the following that all CMEs have flux rope structures and their missing can be explained by observational effects. A further aspect which justifies the assumption of a coronal flux rope morphology is the existence of equally shaped phenomena in the low corona, called "sigmoids". Sigmoids can be observed in soft X-ray (see Figure 4.5) and sometimes in extreme ultraviolet emission as precursor of solar eruptions (Manoharan et al., 1996; Rust \& Kumar, 1996; Hudson et al., 1998). The ascending MHD simulation of a twisted flux rope in Fig. 4.6 is presented to visualise the flux rope character of a sigmoid. Most sigmoids 

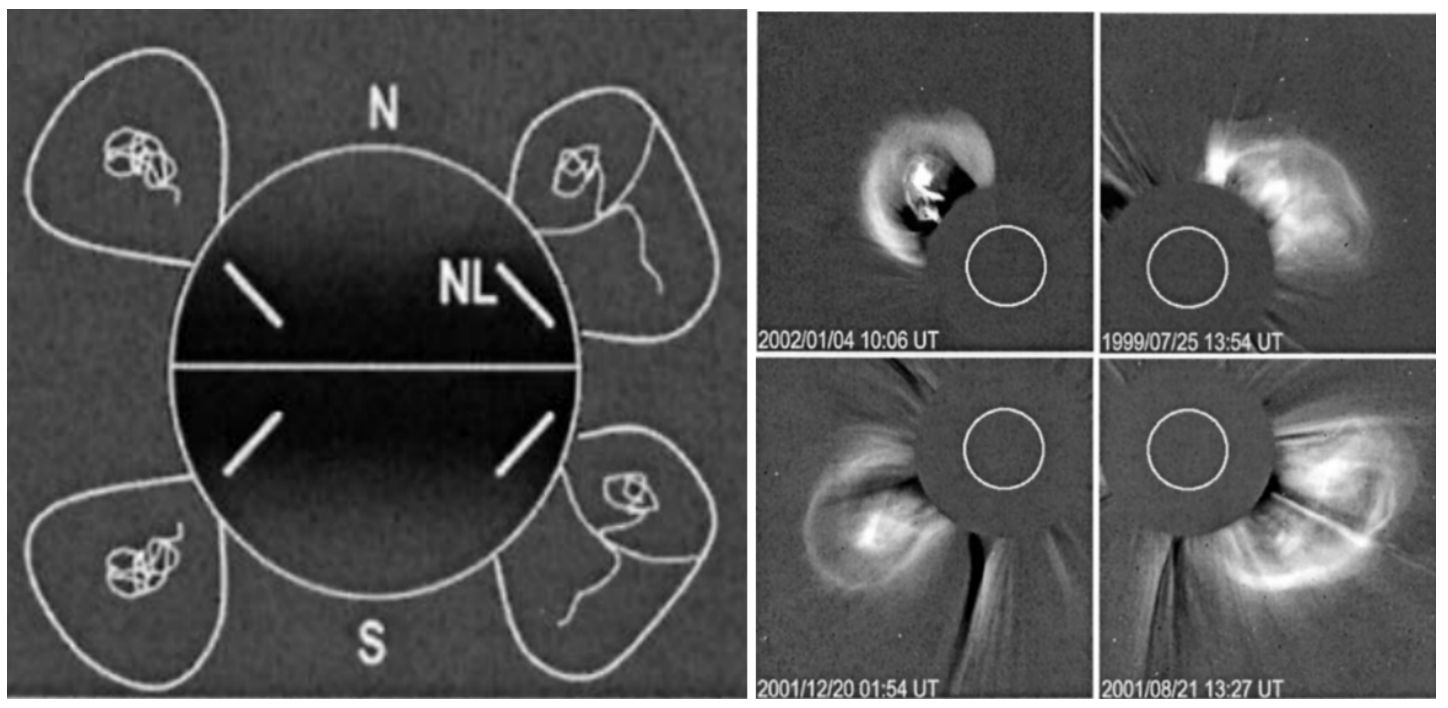

Fig. 4.4: Left: Simplified scheme of CME projection based on a cylindrical magnetic flux rope CME topology. The projections account for the most common magnetical neutral line (NL) configuration in the corresponding quadrant on the solar disk. Right: Four LASCO C2 examples corresponding to the schematic drawing. The coronal cavity is only recognised when the cylindrical axis is aligned with the line of sight. Otherwise the CME is only screened by a bright coronal loop. (Cremades \& Bothmer, 2004)

appear in active regions over curved polarity inversion lines. They can exist in a quiescent stage long before the eruption and are often associated with a filament. Sigmoids generally appear as S-shaped in the northern and as inverse S-shaped in the southern hemisphere, caused by the photospheric shearing as a result of the differential solar rotation. Different sigmoid models are reviewed by Gibson et al. (2006). In the following, the formation of such flux ropes is regarded. Two fundamental theories compete for a proper explanation (see MacTaggart \& Haynes 2014; Filippov et al. 2015; Roussev 2008 and references therein), namely the flux emergence from below and the flux cancellation of stressed arcades. In the "Flux Cancellation Model" the formation of helice-like flux ropes starts from the existence of another very common magnetic field line configuration in the solar corona known as "magnetic loops system" or "arcade" (see Fig. 4.7). Such arcades connect field regions of opposite polarity along the polarity inversion line. The deformation of the photosphere by provoked motions of shear (Kusano, 2005; Aulanier et al., 2012), rotation (Török \& Kliem, 2005) or compression (Amari et al., 2003) can result in magnetic reconnection (Amari et al., 2011; Aulanier et al., 2010) and therefore the formation of magnetic flux ropes (see Fig 4.8).

In the "Flux Emergence Model", a twisted flux rope is already formed in the solar interior. The magnetic field of the flux rope is confined by the shielding of an oppositely directed boundary current (Solovev, 1985) (as presented in Fig 4.10). Due to buoyant forces, the flux rope emerges into the photosphere below a pre-existing coronal field (Manchester et al., 2004; Magara, 2006). In this region, buoyancy forces are not sufficient to carry the flux rope out any further. Simulations on dynamic flux emergence have been carried out by many authors (e.g. Magara \& Longcope 2003; Arber et al. 2007; MacTaggart \& Hood 2009; MacTaggart 2011; Fang et al. 2012). It was proposed that magnetic buoyancy instability could be responsible for further emergence into the corona (Acheson, 1979; Hood et al., 2012). The shielding current spreads far in all directions, except the downward direction where the photosphere forms an analogue mirror current (Kuperus \& Raadu, 1974). Even 


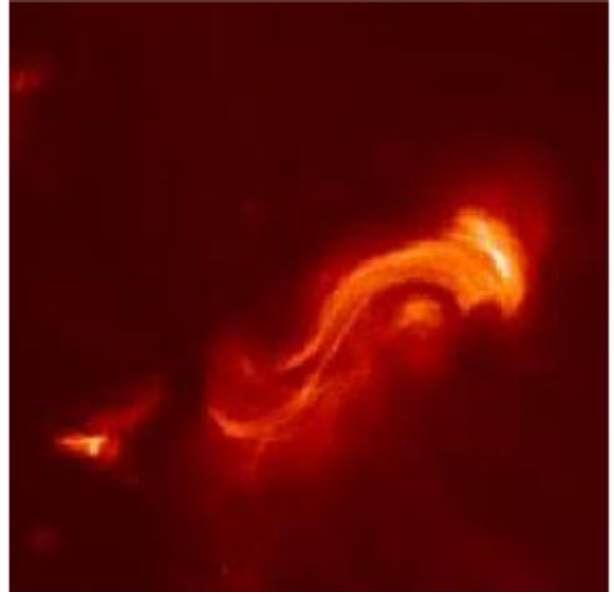

Fig. 4.5: Sigmoids observed by the XRay Telescope (XRT) (Kosugi et al., 2007) onboard the Hinode spacecraft on February 12, 2007. (Golub et al., 2007).

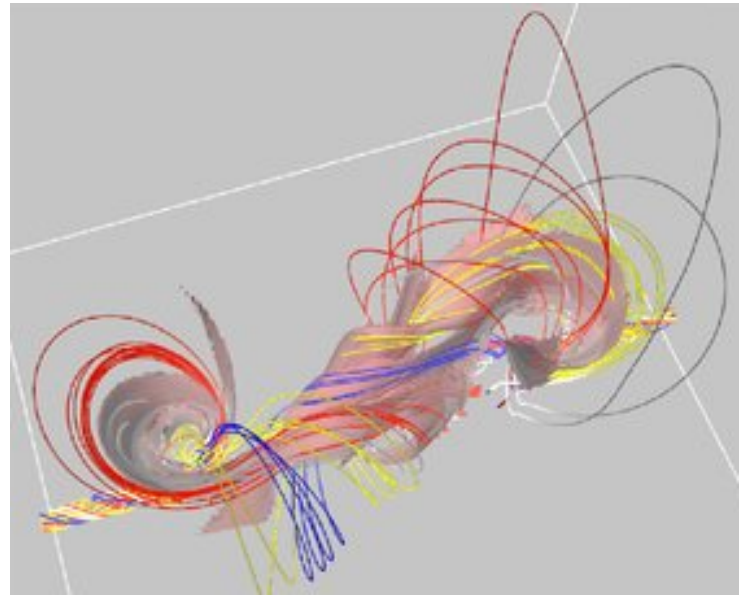

Fig. 4.6: Extract of the MHD simulation of the evolution of the sigmoidal structure. (Archontis et al., 2009)
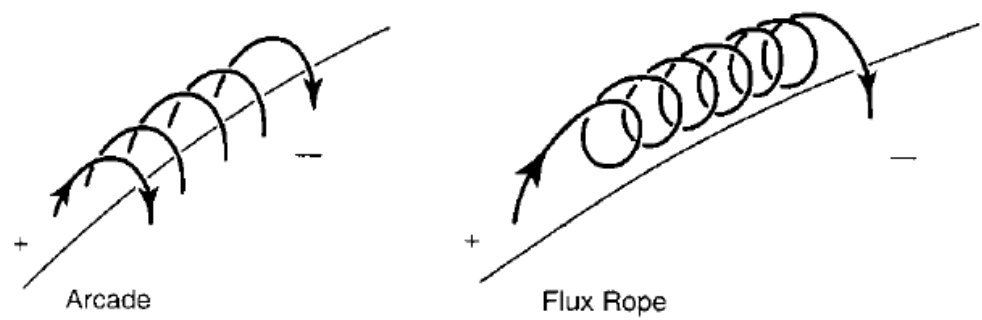

Fig. 4.7: Schematic field line configuration of a coronal arcade and a coronal flux rope over a polarity inversion line. (Klimchuk, 2001)

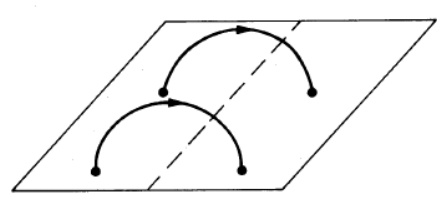

(a)

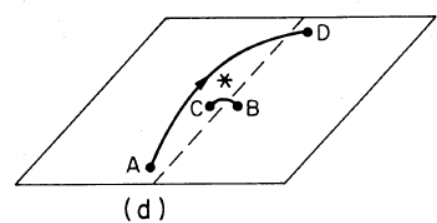

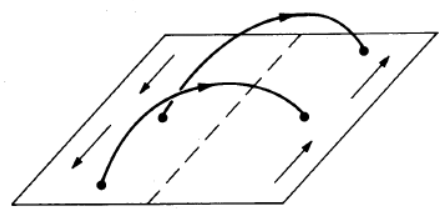

(b)

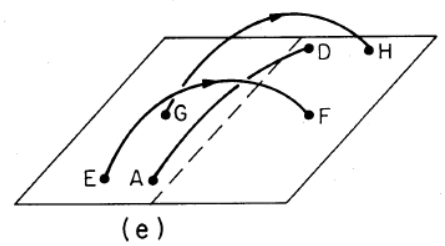

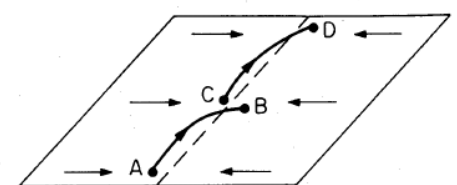

(c)

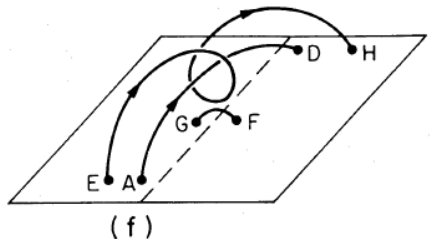

Fig. 4.8: Schematic of coronal flux rope formation due to flux cancellation. The rectangle represents the solar photosphere with the magnetic neutral line (dashed) of an active region separating two regions of opposite polarity. The initial potential field (a) gets sheared due to flows along the neutral line (b). The shearing is further increased by flows towards the neutral line (c). Reconnection between $\mathrm{CB}$ and AD occurs (d). The shorter loop submerges. The overlaying loops EH and EF (e) equally reconnect (f) forming a helical loop EH and a shorter submerging loop GF. (van Ballegooijen \& Martens, 1989) 

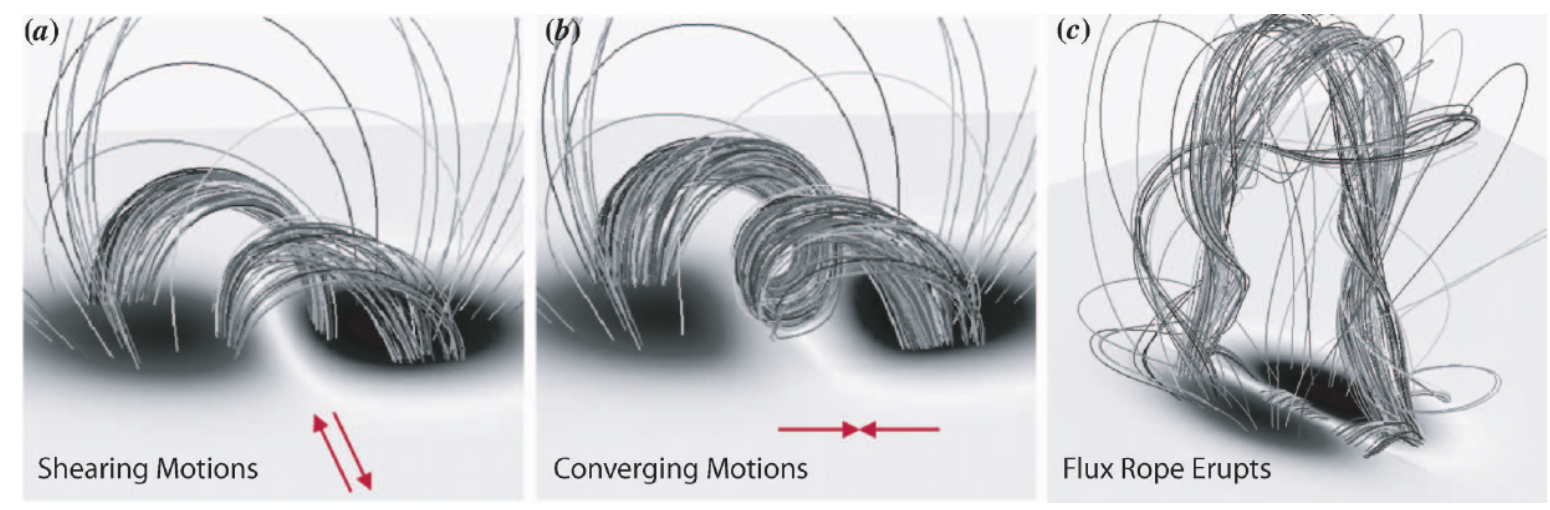

Fig. 4.9: Formation of a flux rope from a magnetic arcade structure. The differential rotation of the solar photosphere leads to a shearing motion of the magnetic neutral line (a). In this "storage" phase free magnetic energy slowly builds up in the overlaying stressed arcades. The reconnection of the sheared field lines leads to the formation of a flux rope (b). In the last step, magnetic cancellation with the overlaying field increases the size and strength of the flux rope. At some point, the overlaying field becomes too weak to counterbalance the flux rope and the eruption is triggered (c). (Roussev, 2008)
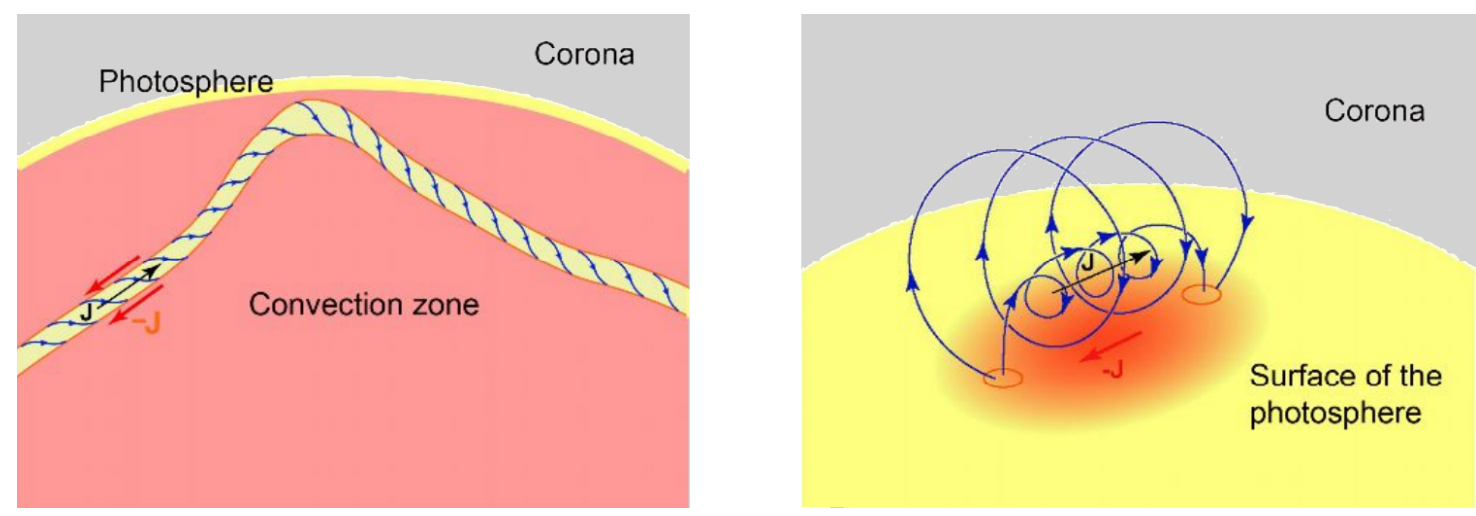

Fig. 4.10: Schematic of the flux emergence model. Inner currents in the solar convection zone form flux ropes which emerge due to buoyancy forces to the surface of the photosphere. (Filippov et al., 2015) 
if the Lorentz forces are not sufficiently strong to raise the flux rope, they can provoke a shearing of the emerged magnetic field along its polarity inversion line (Török et al., 2014). Observations of Okamoto et al. $(2008,2009)$ obtained with the Solar Optical Telescope (SOT) aboard the Hinode space telescope support the theory of flux emergence from below the photosphere along the polarity inversion line.

\subsection{Flux Rope Eruption}

In this section, the eruption mechanisms of coronal flux ropes are discussed. The most common explanation is presented and then followed by further theoretical concepts and models. All these models start from the same initial situation. A coronal flux rope is typically embedded in a larger overlaying magnetic loop structure. Such a combined magnetic field line configuration can be found over active regions and have been investigated with three-dimensional numerical MHD simulations (see Figure 4.11). In Figure 4.12 magnetic field lines are reconstructed above a bipolar magnetic region and show a magnetic flux rope within a magnetic loop structure.

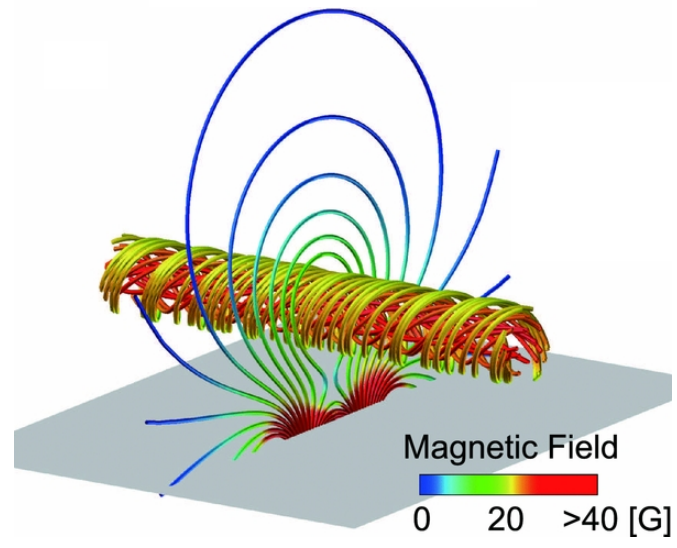

Fig. 4.11: MHD simulation of the initial eruption phase of an idealised coronal flux rope which is embedded in an ambient arcade field. (Nishida et al., 2013)

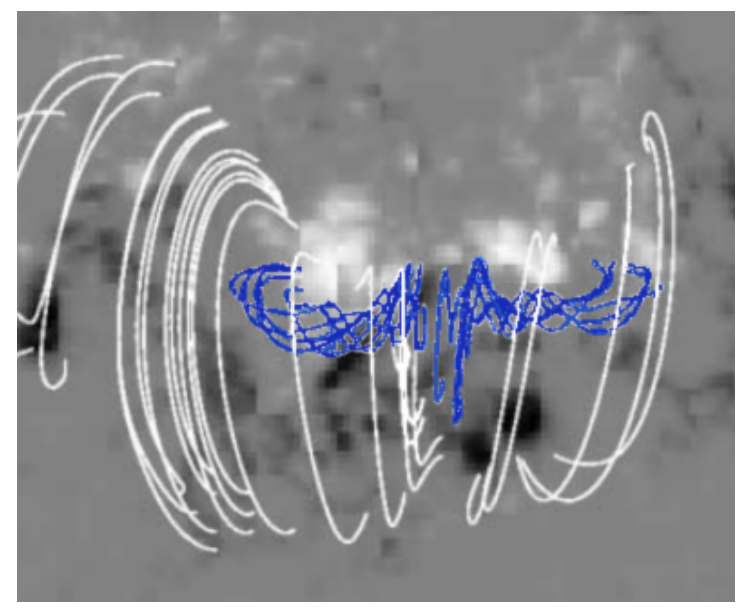

Fig. 4.12: Reconstruction of a magnetic rope (blue) from a photospheric magnetogram. The flux rope is positioned over a magnetic neutral line and embedded in an arcade structure (white). (Adapted from Yan et al., 2001)

Starting from this initial configuration, the eruption of a magnetic flux rope is triggered by the partial reconnection with the overlaying magnetic arcade (see Figure 4.13). The resulting energy release heats the local corona and creates an intense pressure gradient. In the following, this pressure gradient drives an upward motion of the coronal flux rope. In this quasi-force free equilibrium the magnetic pressure and the magnetic tension are counterbalanced by each other. The loss of equilibrium is caused by the dissipation of magnetic tension. Above the flux rope, this effect can be initiated by the further reconnection with the overlaying magnetic field. At some point the magnetic loop system, which is holding the flux rope, is stretched so far that the inner magnetic field lines reconnect below the flux rope (Figure 4.14) leading to a huge abrupt dissipation of magnetic tension and a burst of energy - the solar flare. It is controversially debated if the thermal flare energy contributes significantly to the kinetic energy of the rising flux rope (see Section 4.3 and references 

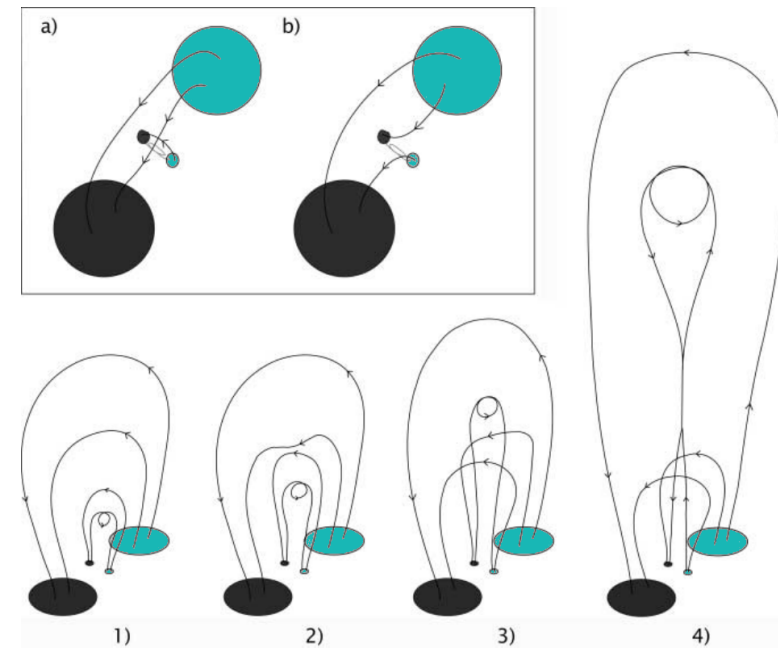

4)

Fig. 4.13: Schematic drawing of a flux rope eruption. Bottom: (1) From a small bipolar region embedded in a large-scale bipolar region, magnetic flux rises under a pre-existing magnetic field loop. (2) The magnetic field lines of the rising flux tube reconnect with the external field lines and (3) open the space for the flux rope to escape. (4) During the expansion the inner magnetic field lines reconnect which leads to a solar flare burst. Top: Reconnection process between phase (2) and (3). (Bemporad et al., 2007)

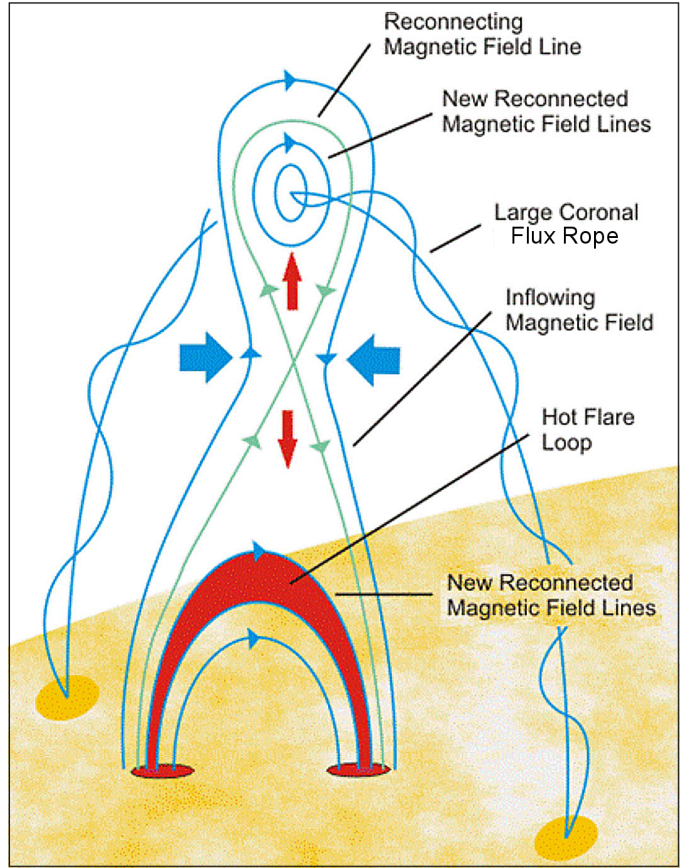

Fig. 4.14: Illustration of an eruptive flux rope with an overlying magnetic loop. During the outward emergence of the flux rope, the reconnection of magnetic field lines below the flux ropes results in a solar flare. (Adapted from Gordon Holman - NASA)

therein). However, it has to be pointed out that flux ropes can also erupt without magnetic reconnection but only due to the loss of the magnetic pressure to magnetic tension equilibrium.

\subsubsection{Tiggering of Flux Rope Eruptions}

The triggering mechanisms of flux rope eruptions are still controversially discussed (Schmieder et al., 2013). A variety of theoretical mechanisms have been proposed over the years. Following the review of Klimchuk (2001), mechanical analogues can be used to categorise the CME triggering mechanism into five basic concepts. These concepts (see also Fig. 4.15) are presented in more detail below.

- Thermal Blast: In this early concept, the flux rope is triggered by the explosive rise of thermal pressure caused by a solar flare. As the flux rope loses its containment by the overlaying closed magnetic field lines, it will erupt. In contrast to the model, observations have shown that the flare, which in many cases does not even exist, is preceded by the erupting CME (Harrison, 1986). Therefore this concept is considered out-dated (Gosling, 1993).

- Dynamo: This concept is based on the real-time stressing of the magnetic field through flux injection. The most likely injection process would come from the emergence of new arch shaped field lines into the corona.

- Mass Loading: The slow build up of mass through, for example, growing eruptive prominences also provokes the buildup of magnetic stress. When some of the mass 


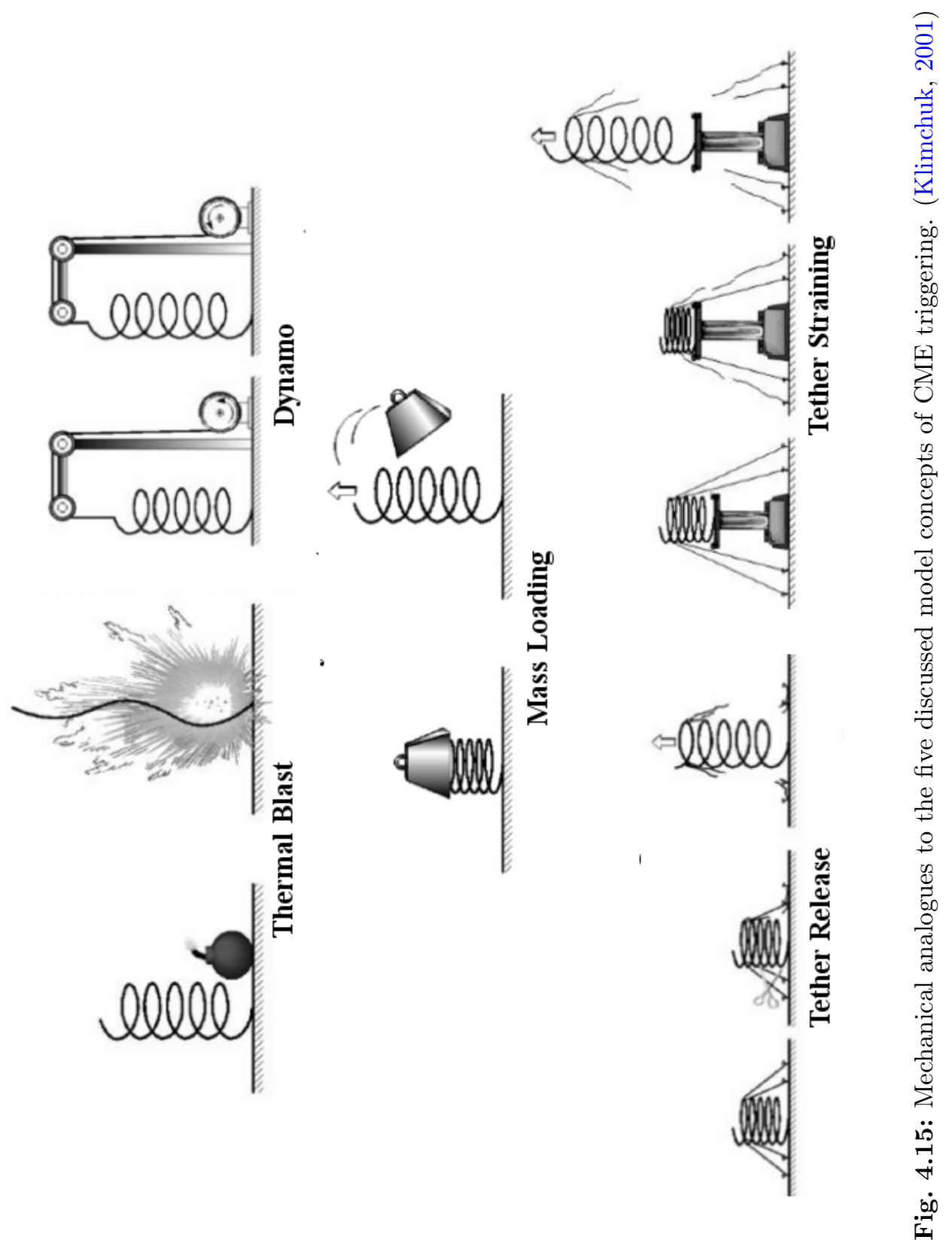


is removed it leads to the eruption of the system. Obviously, this concept can only account for a subset of CMEs (Low, 1996).

- Tether Release: The downward-directed magnetic tension field lines are often referred to as "tethers". Footpoint motion can increase tension in some tethers and they break away. The increased tension on remaining tethers will then lead to an explosive break-off of the remaining tethers.

- Tether Straining: Tether straining models are similar to tether release models, but the tension on the tethers is the result of an external force.
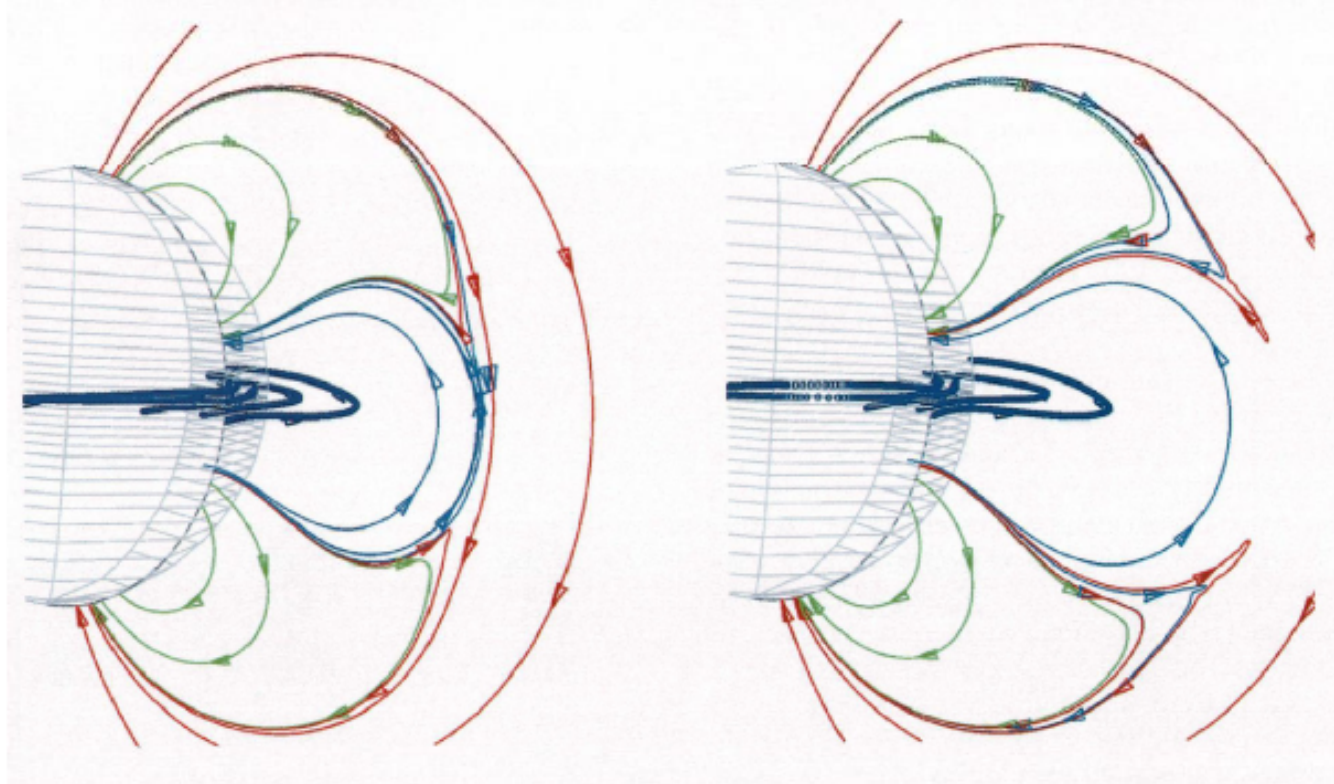

Fig. 4.16: MHD simulation of the Breakout CME model. A quadrupolar structure (blue) is adjacent to two arcades (green). The shearing of the underlying loops (light blue) is increasing the pressure on the overlaying magnetic loops (dark blue) and the global magnetic dipole field (red) which is representing the tethers. The reconnection process triggers and leads to a CME eruption. (Antiochos et al., 1999)

These five concepts are grouped into two different fundamental categories: Directly Driven Models (Thermal Blast, Dynamo) and Storage and Release Models (Mass Loading, Tether Release, Tether Straining). Both compete for a proper description of the necessary energy releasing processes to overcome the balance between magnetic pressure and magnetic tension. The magnetic pressure forces the flux rope to rise in diameter and expand, while magnetic tension acts against the bend of magnetic field lines. The outward pressure gradient needs to increase in order to make the system unstable, increase the force balance over time and provoke an eruption. Currently, there is no indication that the necessary energy comes from below the corona, so it has to be earlier stored in the corona itself. This is a major issue with directly driven models. The conversion of overlaying closed into open magnetic field lines would actually increase the magnetic energy of the field instead of draining energy from it (Aly, 1984, 1991; Sturrock, 1991). The solution for this problem is only treated in storage and release models. Free magnetic energy is slowly building up in a stressed magnetic field configuration by footpoint shearing or the accumulation of mass or both. The energy is then released and converted during the eruptive phase. Further noteworthy models are the "Quadrupolar Breakout Model" (Antiochos 
et al., 1999) (Figure 4.16), the "Torus Instability Model" (Aulanier et al., 2010) or "Kink Instability Models" (Török \& Kliem, 2004; Williams et al., 2005). However, all these models are simplifications for very idealised local magnetic field line configurations. The high complexity and diversity of real magnetic line configurations can account for more than one possible triggering mechanism or even combinations of them.

\subsection{Solar Flares}

A solar flare is a sudden burst of X-ray and UV radiation caused by magnetic reconnection on the solar surface, which can affect all atmospheric layers of the Sun. Solar flares are classified by their X-ray intensity into the categories A,B,C,M,X as presented in Table 4.1.

\begin{tabular}{|c|c|}
\hline Classification & Peak Flux $\left[\mathrm{W} / \mathrm{m}^{2}\right]$ \\
\hline A & $\leq 10^{-7}$ \\
B & $10^{-7}-10^{-6}$ \\
C & $10^{-6}-10^{-5}$ \\
M & $10^{-5}-10^{-4}$ \\
X & $\geq 10^{-4}$ \\
\hline
\end{tabular}

Table 4.1: Solar flare X-ray classification of X-ray peak flux at $1-8 \AA$. The classes A-M can be subdivided by a linear scale from 1 to 9 .

An example of a M9.2 flare is shown in Figure 4.17. Typical energy releases of solar flares are in the order of $1 \times 10^{20}$ joules. Major events can even release up to $1 \times 10^{25}$ joules of energy. During the radiation emission phase, the local coronal plasma is heated to a few million kelvin. According to NASA, one of the most powerful earth directed flares was the X28 class "Halloween" flare on October 10, 2003 (see Fig. 4.18) which had an energy release of $6 \times 10^{25}$ joules, the equivalent of 6 billion megatons of TNT. The energy limit of such super flares on our Sun is likely ten times higher, but in Sun-like stars they might even reach about $10^{29}$ joules (Aulanier et al., 2013).

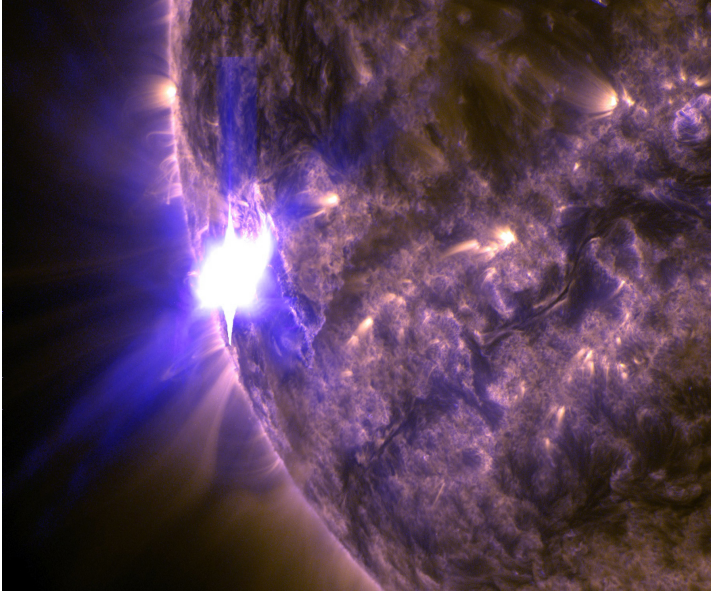

Fig. 4.17: Flares at the solar limp like this M9.2 event on March 7, 2015 (Fig. 4.17) captured by SDO/AIA in a merge of different extreme ultraviolet light wavelengths. (NASA SDO)

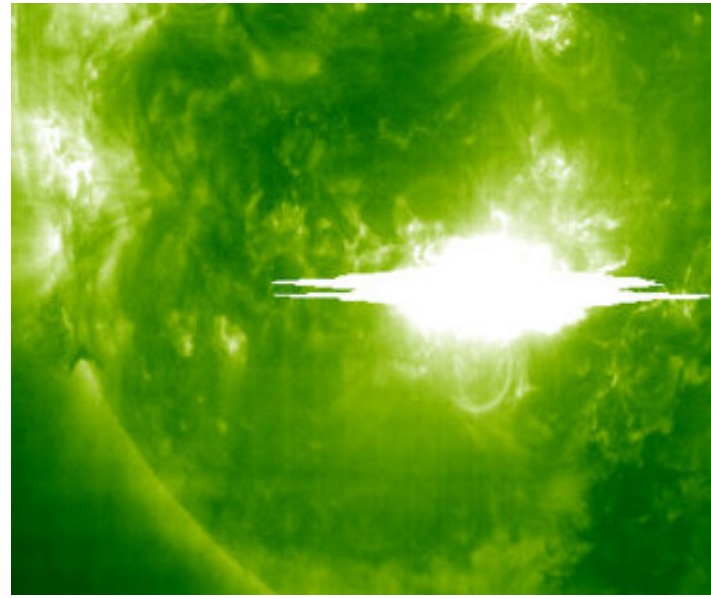

Fig. 4.18: X28-class solar flare on October 10, 2003 captured by SOHO/EIT in extreme ultraviolet light of the wavelength $195 \AA$. Brodrick et al. (2005) suggests, that this events even has to be characterised as X40 flare and is therefore comparable to the Carrington flare. (ESA/NASA - SOHO) 
CMEs historically have been regarded as coronal response to a flare (Harrison, 1991). They can be considered as different manifestations of the same physical process, namely the conversion of magnetic free energy to radiative and kinetic energy (Chen, 2011). During the reconnection phase, open coronal magnetic field lines are closed, resulting in the conversion of inherent magnetic energy. A solar flare loop is formed, emitting radiation at its footpoints and loop peak, visible on timescales from minutes to hours on the solar disc. On September 1, 1958 Carrington (1859b) discovered the first solar flare, which was followed a day later by an intense geomagnetic storm. Flares were therefore causally linked to geomagnetic storms, an idea which was corrected later with the discovery and deeper understanding of CMEs. In the standard reconnection flare model CSHKP (Carmichael, 1964; Sturrock, 1966; Hirayama, 1974; Kopp \& Pneuman, 1976) solar flares are driven by a flux rope eruption. The solar flare is then triggered by magnetic reconnection in the magnetic cavity beneath the flux rope (see Fig 4.19). Some authors conclude that

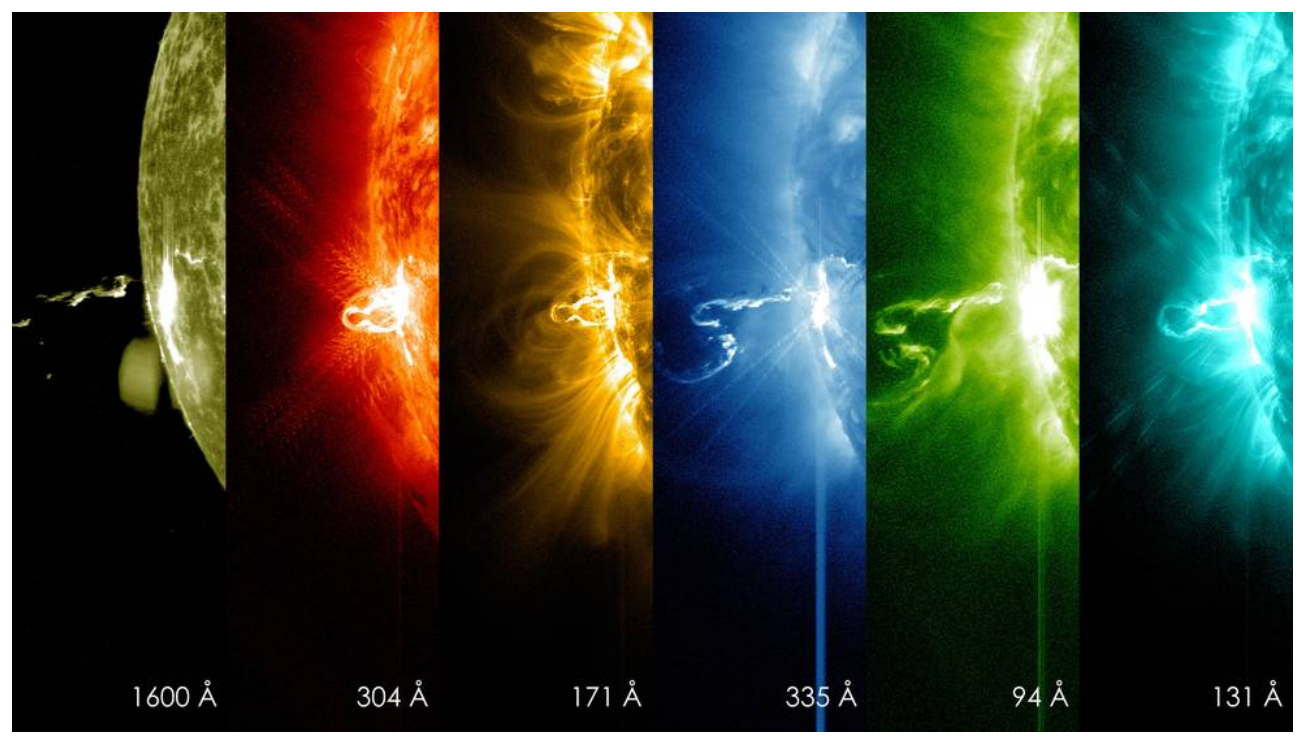

Fig. 4.19: First moments of an X4.9-class flare observed in different wavelengths by SDO on February 24, 2014. The flare is triggered by a coronal flux rope, which transports hot coronal material out of the Sun's atmosphere. (NASA/SDO/Goddard's Scientific Visualization Studio)

CMEs are partially flare driven, as flare-associated CMEs tend to be faster than non-flareassociated CMEs (MacQueen \& Fisher, 1983). Many of such cases, where a solar flare is preceded by an associated CME, have been found (Forbes et al., 2006) since then. Kahler (1992) showed that associated CMEs have a reasonable confidence to originate from flare regions for very intense events. Their interaction with CMEs is still not fully understood as the correlation between solar flare X-ray intensities and CME energies is weak $(R=0.53)$ (Hundhausen, 1999; Moon et al., 2002b). On the other hand, Andrews (2003) statistically proved that strong flares are more likely related to CMEs, yet 40 percent of M-class flares have no CME association. CME eruption is therefore clearly not the only trigger for magnetic reconnection leading to solar flares. Green et al. (2002) showed an alternative cause in the analyses of a X1.2 flare, where the origin of the flare was the interaction of two pre-existing coronal loops. 


\subsection{Post Eruptive Arcades}

Post Eruptive Arcades (PEA), sometimes also called post-flare loops, are generally formed after the eruption of a filament (discussed in Section 4.5) in conjunction with a solar flare. The arcade is formed by the erupting flux rope magnetic field lines beneath the solar flare reconnection point, accordingly to the CSHKP magnetic reconnection model. Figure 4.20 shows a post eruptive arcade on the Sun's western limb. The pre-dominant emission of accelerated electrons of the solar flare, injects hot coronal plasma and magnetic fields. The result is an apparent flickering glowing in the ultraviolet spectrum of about 2 to 20 hours. Within the magnetic confines of the arcade, the plasma is isolated from the very hot coronal plasma and can cool down to much lower temperatures. Besides coronagraph observations and disappearing filaments (Webb \& Hundhausen, 1987; Bothmer \& Schwenn, 1994), PEAs are very reliable tracers for CME eruptions (Tripathi et al., 2004).

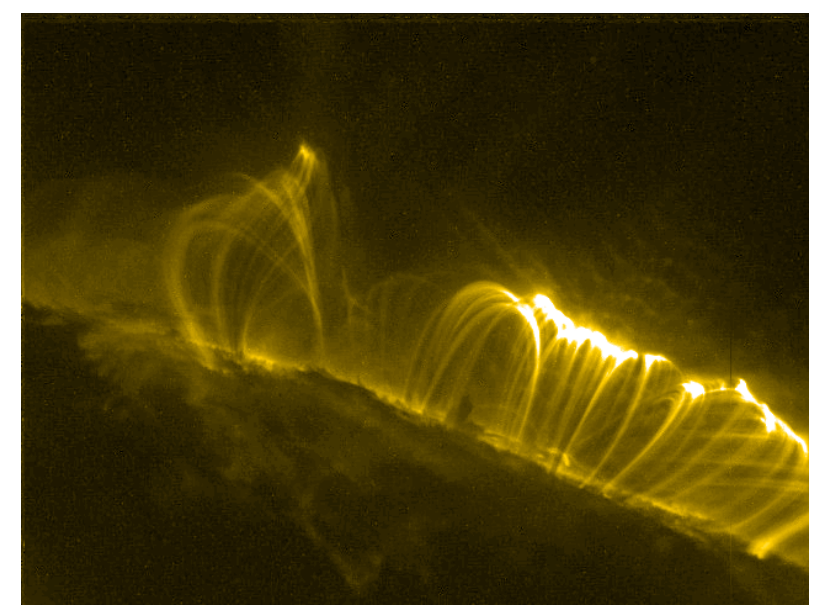

Fig. 4.20: Post eruptive arcade observed in $195 \AA$ by the Transition Region and Coronal Explorer (TRACE) on November 4, 2003 at 19:34 UT. The arcade loops appeared three hours after a X28 flare. Hot coronal plasma injected into the arcade structure during the flare phase causes them to glow for several hours. (NASA/TRACE)

\subsection{Prominences}

Prominences are loops of dense $\left(10^{10}-10^{11} \mathrm{~cm}^{3}\right)$ and cold $\left(\sim 10^{4} \mathrm{~K}\right)$ chromospheric material which is captured in a strong coronal flux rope (Priest et al., 1989). Such loops can have lengths of up to $1 \mathrm{R}_{\odot}$. They build up gradually over days and may persist in the corona over weeks. Prominences are pulled out by the magnetical forces against the solar gravity into the hotter tenuous corona. If a prominence is observed in the center of the solar disk their projection manifests in absorption rather than in emission so that the same physical phenomena became visible as a dark thin "filament" (see Fig 4.21) in the emission spectrum of 304 UV light. The first description of a prominence was found in the Russian "Chronicle of Novgorod" and dates to the solar eclipse on the 1 May 1185 (Sviatsky, 1923; Hetherington, 1996): "In the evening there was an eclipse of the Sun. It was getting very gloomy and stars were seen ... The Sun became similar in appearance to the Moon and from its horns came out somewhat like live embers. "It was not before 1868 when Janssen and Lockery independently developed a method to observe prominences in full daylight by the use of spectroscopes in $\mathrm{H} \alpha$ leading to a rapid increase of our knowledge about them (Slocum, 1912). The encapsulated plasma often does not fill the entire flux rope but only a lower part of it (Priest et al., 1989; Ridgway \& Priest, 1993) as demonstrated in Fig (4.22). In that case the helical geometry is not fully revealed. During filament activation, plasma can spill over the upper parts of the flux tubes. Visual observations confirm a twisting helical rotation movement around the flux rope axis (Wang \& Stenborg, 2010; Joshi et al., 


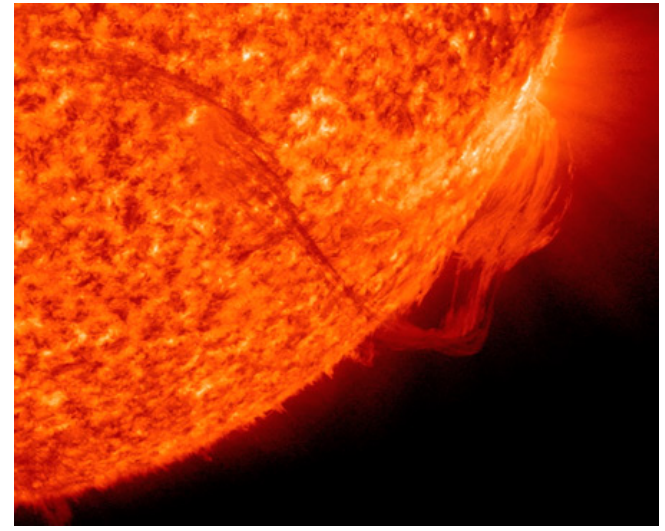

Fig. 4.21: Extremely long and noneruptive prominence observed by SDO in $304 \AA$ on November 17,2011 . (NASA)

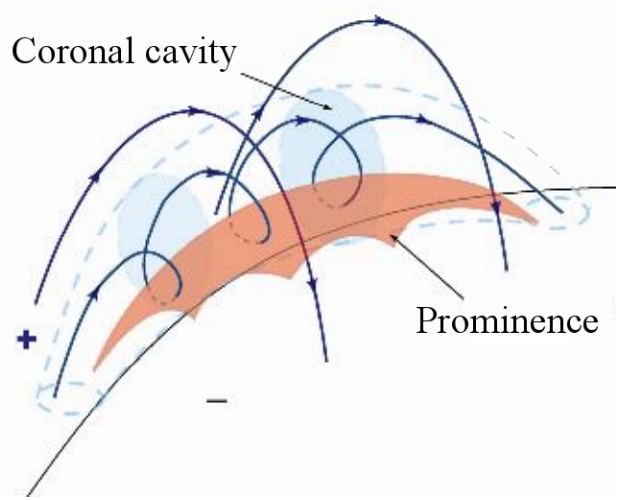

Fig. 4.22: Scheme of a coronal flux rope holding coronal plasma. (EIT Consortium)

2014; Patsourakos et al., 2013; Cheng et al., 2014).

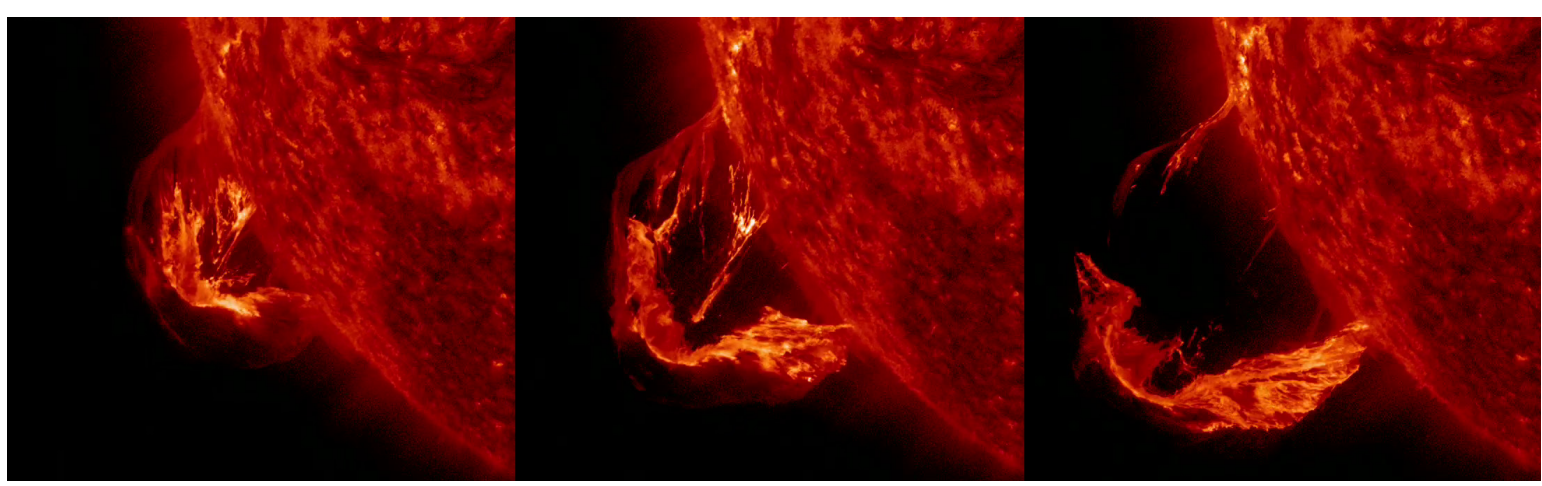

Fig. 4.23: Prominence eruption as part of a coronal mass ejection observed in $304 \AA$ of extreme ultraviolet light over three hours on February 24, 2015. (SDO/NASA)

In most cases prominences slowly fade away but sometimes they erupt and form CMEs (Gopalswamy et al., 2003a; Jing et al., 2004). Figure 4.23 displays the eruption of a prominence which is associated to a CME. The eruption of the flux rope pushes the overlying magnetic field loops upwards which then shape the CME frontal loop. Prominence eruptions require a destabilising element. Jing et al. (2004) showed in a study of over 100 prominences that in $68 \%$ of all cases, the destabilisation was provoked by new magnetic flux emerging from the photosphere. Gopalswamy et al. (2003b) investigated a set of 226 events between January 1996 and December 2001. They found that $72 \%$ of all prominence eruptions and $83 \%$ of all radial events are associated with CMEs. Also prominence eruptions without CMEs are very slow, occur close to the solar surface and erupt parallel to the limb.

\subsection{Coronal Shock Waves}

Shock waves are frequently occuring phenomena in the solar corona and heliosphere. Their existence in the corona was first proposed by Wild et al. (1963). Shortly after, they were detected with in-situ measurements of the Mariner 2 space probe (Sonett et al., 1964). The first indirect, but significant proof of coronal shock waves followed from observed deflections 
of coronal solar streamers (Gosling et al., 1974; Sheeley et al., 2000b). Soon afterwards, white light coronagraphs revealed a connection of interplanetary shocks to coronal mass ejections and solar flares (Gosling et al., 1975; Sheeley et al., 1985; Schwenn, 1986). Lowfrequency radio instruments even allowed to track them from the Sun to Earth (Cane et al., 1982). The first density enhancement from a CME driven shock were detected with the LASCO coronagraphs (Vourlidas et al. 2003). A similar event is presented in Figure 4.24. Coronal shock waves can be subdivided into two categories: Blast wave types
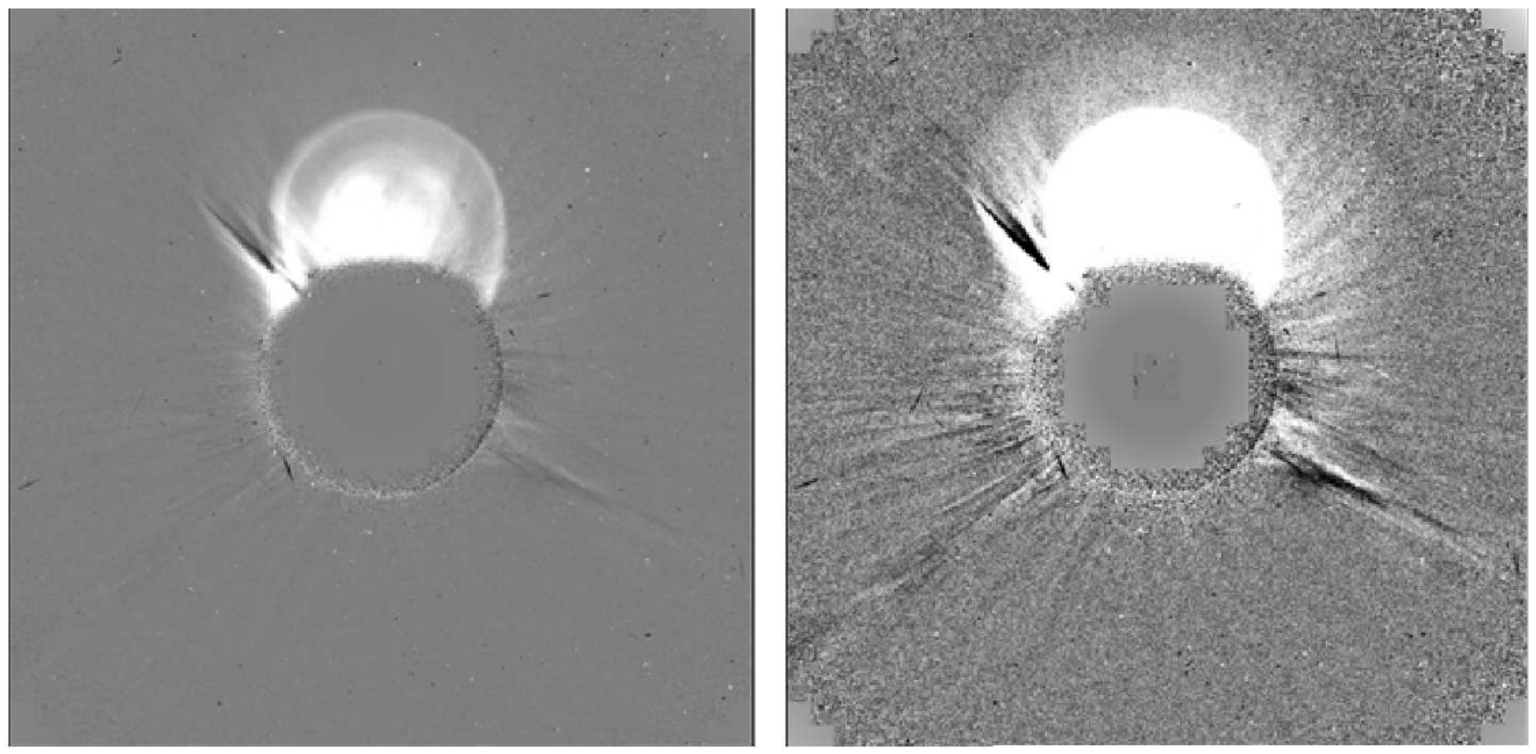

Fig. 4.24: White-light appearance of a LASCO/C 2 CME. Left: The CME shows a clear threepart structure. Right: With higher contrast a faint shock front becomes visible. (Vourlidas et al., 2013)

are produced by huge pressure pulses during a solar flare, whereas piston shock waves are driven by CMEs. The characteristics of the piston shock depends on the CME morphology, so that more narrow CMEs create bow shocks and wider CMEs have the characteristics of an expansion shock wave (Kahler \& Gopalswamy, 2009). Perturbations travel through a magnetised plasma with the Alfvén speed, which is given by

$$
v_{\mathrm{A}}=\frac{B_{0}}{\sqrt{\mu_{0} \rho_{0}}},
$$

where $B_{0}$ is the unperturbed magnetic field, $\mu_{0}$ is the magnetic permeability and $\rho_{0}$ is the mass density of the medium. Usually, CMEs have to exceed the Alfven speed in the corona $\left(v_{A} \approx 500-1500 \mathrm{~km} / \mathrm{s}\right)$ in order to form a bow shock wave. However, wider CMEs with speeds below the Alvén speed can also create shock waves (Žic et al., 2008; Vršnak \& Cliver, 2008). They are capable of accelerating charged particles up to high energies and are therefore considered to be the main source of SEPs (Klecker et al. 2006 and references therein) but have only been detected at $1 \mathrm{AU}$ for CMEs with widths greater than 60 degrees (Kahler \& Gopalswamy, 2009). 



\section{Observation \& Instrumentation}

This chapter is designated to the instruments and spacecrafts which are relevant for scientific studies made in this thesis. It presents their design concepts, schematics, observation ranges and positions in the solar orbit. The interplay and the synergies between the different instrument observations is demonstrated via an illustrative sample event. The chapters ends with an overview of the most important heliographic and heliocentric coordinate systems used in this work for the observations of CMEs.

\subsection{Coronagraphs}

The invention of coronagraphs has enabled the discovery of CMEs. Before, the solar corona could only be observed with the naked eye during a solar eclipse due to the much higher intensity of the photospheric light. With an average duration of only 7 minutes, eclipses do not last long enough to observe the transient nature of CMEs. However, this can be achieved with coronagraphs, which are astronomical telescopes designed to imitate a solar eclipse by blocking the photospheric light. The first coronagraph was concepted by Bernard Lyot in 1930 (Lyot, 1939). His design of an externally occulted Lyot coronagraph is presented in Figure 5.1. The main difference to internal Lyot coronagraphs is, that the occulter is positioned before instead of behind the objective lens. Both configurations have different advantages and disadvantages. Externally occulted coronagraphs cannot image the inner corona due to the diffraction limit. Internally occulted coronagraphs are affected by scattering light, as the front lens and aperture are directly illuminated by the solar disk. One big challenge for coronagraphic CME observations is the stray light from the Sun, which cannot be removed by the Lyot principle. According to the Thomson Scattering theory the CME white-light brightness is highly polarised whereas stray light is largely unpolarised. The latter can therefore be greatly reduced by making polarised observations in three states of linear polarisation and calculating the polarised brightness. To obtain polarised brightness images, a sequence of three linearly polarised images is taken. The sequence needs to be taken in short time in order to minimize smearing caused by CME motion. A moderately fast CME moving at the speed of $750 \mathrm{~km} / \mathrm{s}$ would traverse a COR 2 pixel in 15 seconds. For that reason, modern coronagraphs have additional baffles to reduce internal straylight. Furthermore, polarisers are employed to separate the polarised CME light from the unpolarised coronal light. This concept is used in the SOHO/LASCO C2 and C3 coronagraphs (Brueckner et al., 1995a).

\subsection{Solar and Heliospheric Observatory (SOHO)}

The Solar and Heliopspheric Observatory (SOHO) (Domingo et al., 1995) was launched in 1995 as joint mission of the European Space Agency (ESA) and U.S. National Aeronautics 

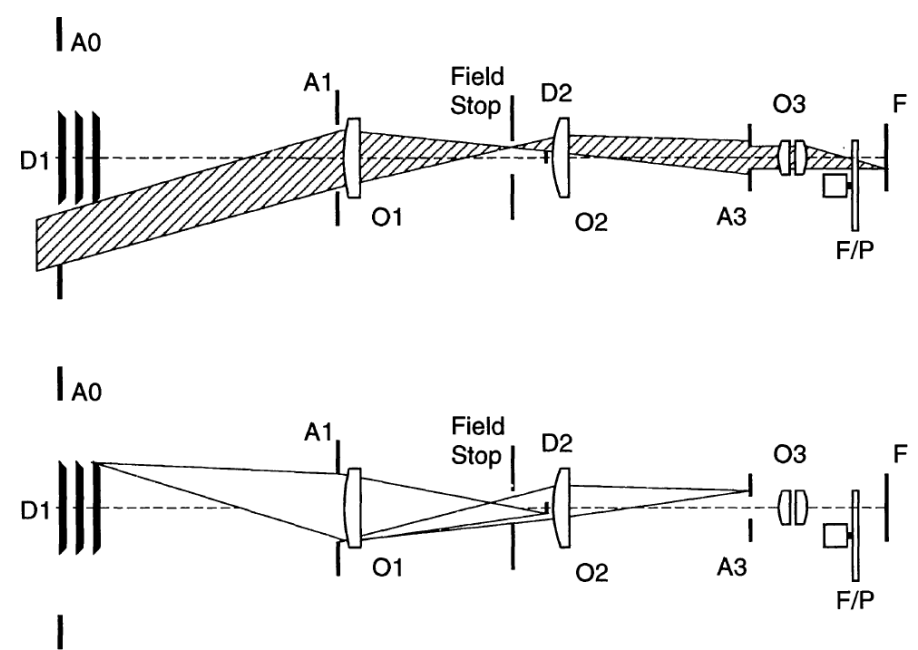

Fig. 5.1: Design concept of the externally occulted Lyot coronagraph C2 on-board of the SOHO spacecraft. In the top row, the path of rays passes from left to right through the coronagraph. The first optical instrument is the external occulter, which blocks of the light from the solar disk, while the coronal light passes around. Directly behind lies the objective lens (O1) which is highly polished to reduce scattering. The lens forms an image of the external occulter onto the internal occulter (D2). The light then passes through the "Lyot Stop" (A3), blocking the light diffracted by the edges of the pupil. The final coronagraph image is formed by the camera objective (O3) onto the focal plane $(\mathrm{F})$. In the bottom row the suppression of straylight by the lyot stop is demonstrated. (Brueckner et al., 1995a).

and Space Administration (NASA). The spacecraft is positioned in the Lagrange Point 1 along the Sun-Earth line, approximately 1.5 million kilometers away from Earth, allowing continuous monitoring of the Sun. The scientific goals of the missions are to study the solar interior, the heating of the corona and the solar wind. The schematic of the SOHO spacecraft is presented in Figure (5.2). The spacecraft is equipped with twelve complementary instruments, three of which are designated to helioseismological probing, three to in-situ measurements of solar plasma and six telescopes with imagers or spectroscopes to investigate the solar disk and atmosphere.

\subsubsection{Large Angle and Spectrometric Coronagraph (LASCO)}

The LASCO (Brueckner et al., 1995a) instrumentation set onboard the SOHO spacecraft consists of three coronagraphs, designated to image the corona in the FOVs of $1.1-3 \mathrm{R}_{\odot}$ $(\mathrm{C} 1), 1.5-6.0 \mathrm{R}_{\odot}(\mathrm{C} 2)$ and $3.7-30 \mathrm{R}_{\odot}(\mathrm{C} 3)$ with an overall image cadence of $30 \mathrm{~min}$. The $\mathrm{C} 1$ coronagraph has an internally occulted reflective design. It is not operational since the loss and recuperation of the SOHO spacecraft in 1998. The design of the C2 and C3 coronagraphs is based on an externally occulted Lyot coronagraph. The obtained total brightness $B_{\mathrm{r}}$, and polarised brightness $p B_{\mathrm{r}}$ are given by the following equations from Billings (1966):

$$
\begin{aligned}
B_{\mathrm{r}} & =\frac{2}{3}\left(I_{\mathrm{a}}+I_{\mathrm{b}}+I_{\mathrm{c}}\right) \\
p B_{\mathrm{r}} & =\frac{4}{3}\left[\left(I_{\mathrm{a}}+I_{\mathrm{b}}+I_{\mathrm{c}}\right)^{2}-3\left(I_{\mathrm{a}} I_{\mathrm{b}}+I_{\mathrm{a}} I_{\mathrm{c}}+I_{\mathrm{b}} I_{\mathrm{c}}\right)\right]^{1 / 2} .
\end{aligned}
$$

Both coronagraphs are equipped with various filters in the range of $400-850 \mathrm{~nm}$ for $\mathrm{C} 2$ and $400-1050 \mathrm{~nm}$ for $\mathrm{C} 3$ as well as three polarisers at $\mathrm{a}=-60^{\circ}, \mathrm{b}=0^{\circ}$ and $\mathrm{c}=+60^{\circ}$. Images 


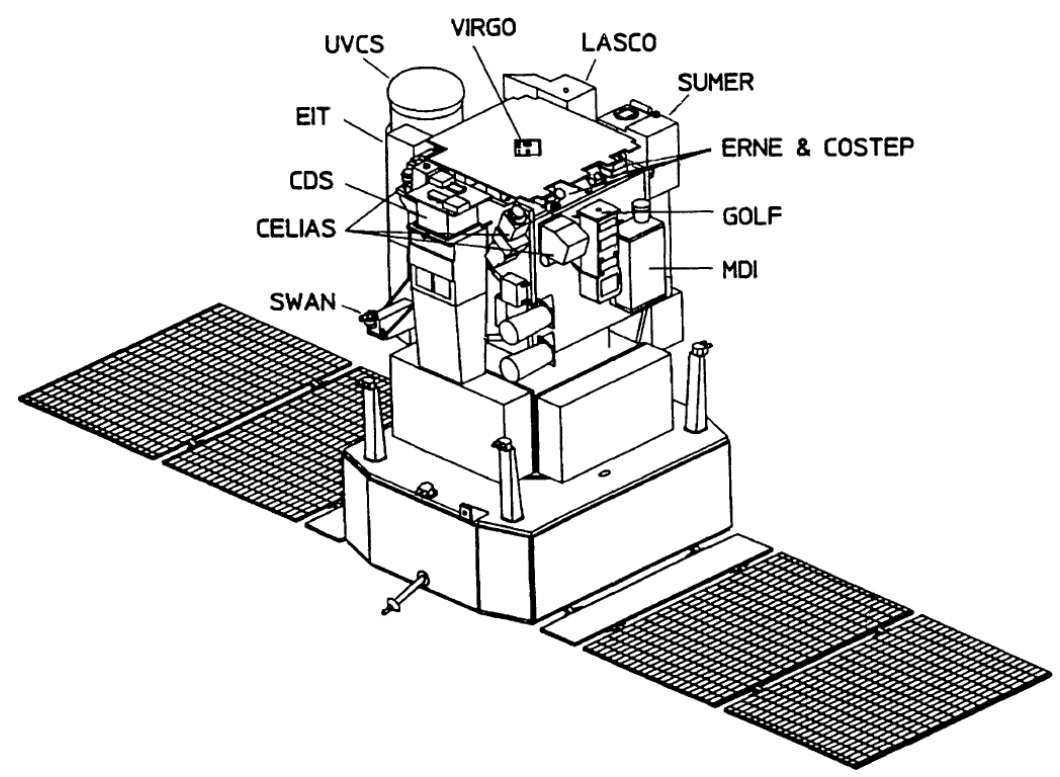

Fig. 5.2: Schematic of the SOHO spacecraft together with the on-board instruments. (Domingo et al., 1995)

are taken with only small time shifts of $15 \mathrm{sec}$ for each polariser to avoid a smearing of fast moving CMEs, which can exhibit velocities of up to $3000 \mathrm{~km} / \mathrm{s}$.

\subsection{Solar Terrestrial Relations Observatory (STEREO)}

The STEREO mission (Kaiser et al., 2008) by NASA consists of two nearly identical spacecraft launched in 2006 into a solar orbit. The goal of the mission is to study the Sun and space weather, including coronal mass ejections and the identification of the critical forces that control their propagation. STEREO A is positioned in an orbit a little closer to the Sun at $0.96 \mathrm{AU}$ and STEREO B a bit further away at $1.04 \mathrm{AU}$, which results in STEREO A pulling further ahead of Earth and B falling successively behind. The spacecraft separates about $\sim 22^{\circ}$ per year from Earth. The positions of the spacecraft are shown in Figure (5.3) for each year since the launch of the mission.

The optical, radio, in-situ particle and field instruments serve to measure solar wind properties such as velocity, temperature and density. They are subdivided into four suits:

- Sun-Earth Connection Coronal and Heliospheric Investigation (SECCHI) (Howard et al., 2008b);

- In situ Measurements of PArticles and CME Transients (IMPACT) (Luhmann et al., 2008);

- PLAsma and SupraThermal Ion Composition (PLASTIC) (Galvin et al., 2008);

- Stereo/WAVES

(S/WAVES) (Bougeret et al., 2008).

In Fig 5.4, the STEREO B spacecraft is presented together with its equipped instrumentation. In particular, the simultaneous usage of both STEREO perspectives enables to deproject the 3-dimensional morphology of CMEs. 


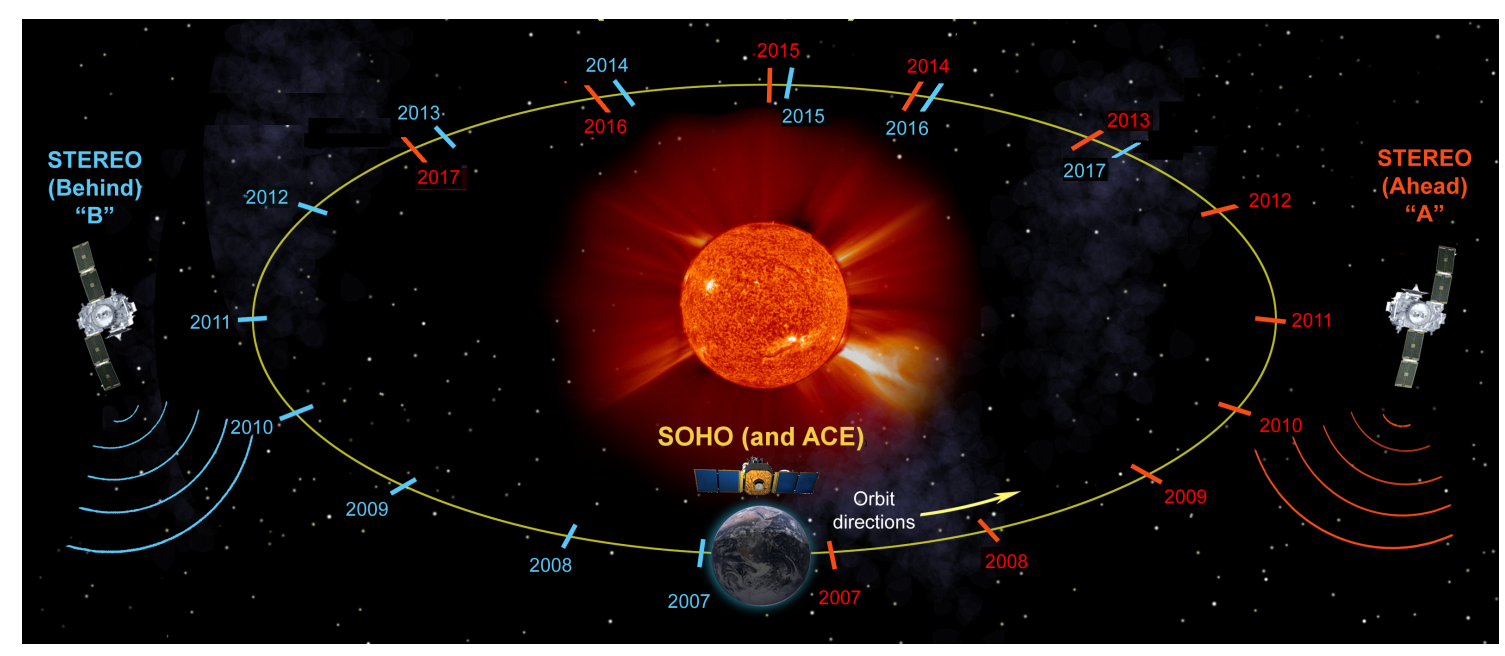

Fig. 5.3: Relative orbital positions of both STEREO spacecraft for each year from June 2007 to June 2017. (Adapted from NASA - STEREO Consortium)

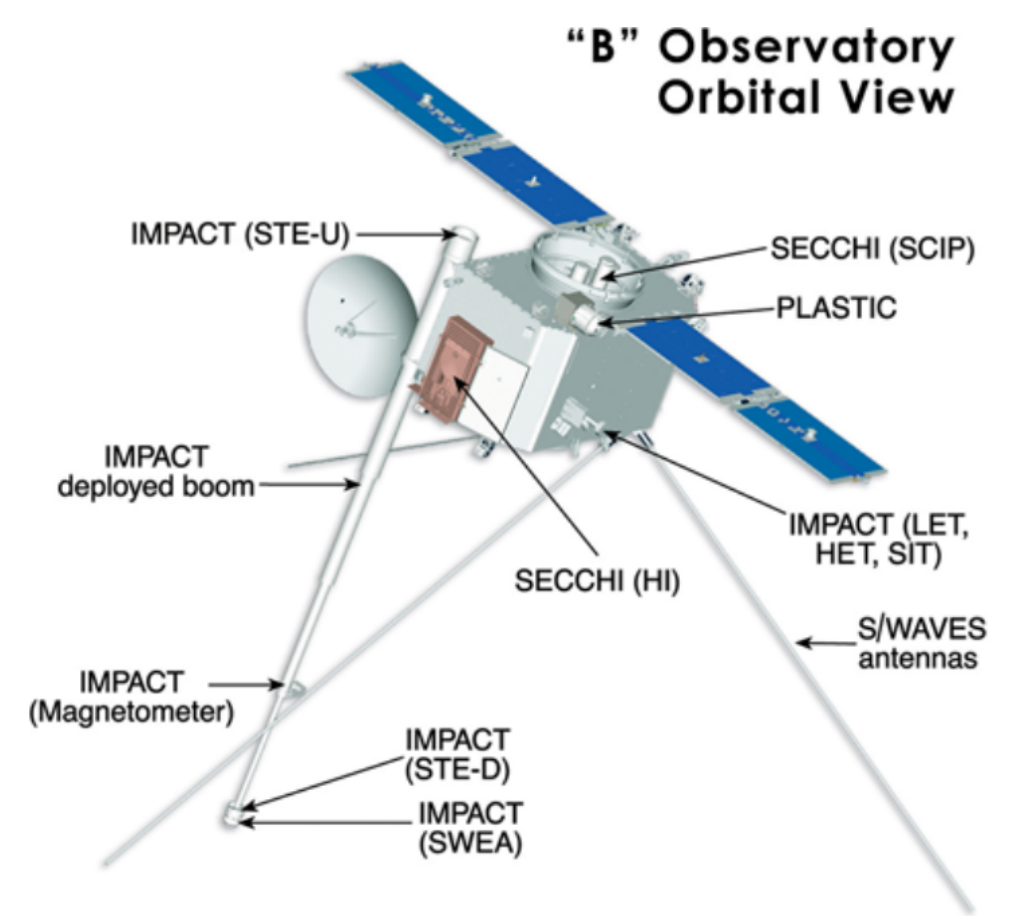

Fig. 5.4: Schematic of the STEREO B spacecraft and its equipped instrumentation. There are slight differences between the STEREO A and B spacecraft, mainly because the spacecraft are flying upside down relative to each other. In order to always point their high gain antennas towards Earth, the arrangement of the long antennas, dedicated to solar wind in-situ measurements, has to be altered. (Kaiser et al., 2008) 


\subsubsection{Sun Earth Connection Coronal and Heliospheric Investigation (SECCHI)}

The remote sensing optical suite SECCHI consists of an Extreme Ultraviole Imager (EUVI) with four observational wavelengths ( $171 \AA, 195 \AA, 284 \AA$ and $304 \AA)$ two coronagraphs COR 1 and COR 2, and two Heliospheric Imagers (HI 1 and HI 2) (Eyles et al., 2009). The combined instrumentation allows the 3-dimensional tracking of CME white-light signatures from close to the Sun to Earth, which facilitates the association of CMEs to CME signatures measured in-situ at L1. The STEREO coronagraphs (COR) are based on the LASCO coronagraph design. COR 1 is an internally occulted Lyot refractive design. The external occulter design of COR 2 is presented in Figure 5.5. They have an observational range in the plane-of-sky of $1.4-4.0 \mathrm{R}_{\odot}(\mathrm{COR} 1)$ and $2.5-15 \mathrm{R} \odot(\mathrm{COR} 2)$, with a time-resolution of $5 \mathrm{~min}$ and $15 \mathrm{~min}$ respectively (Howard et al., 2008b). The photometric response of COR A

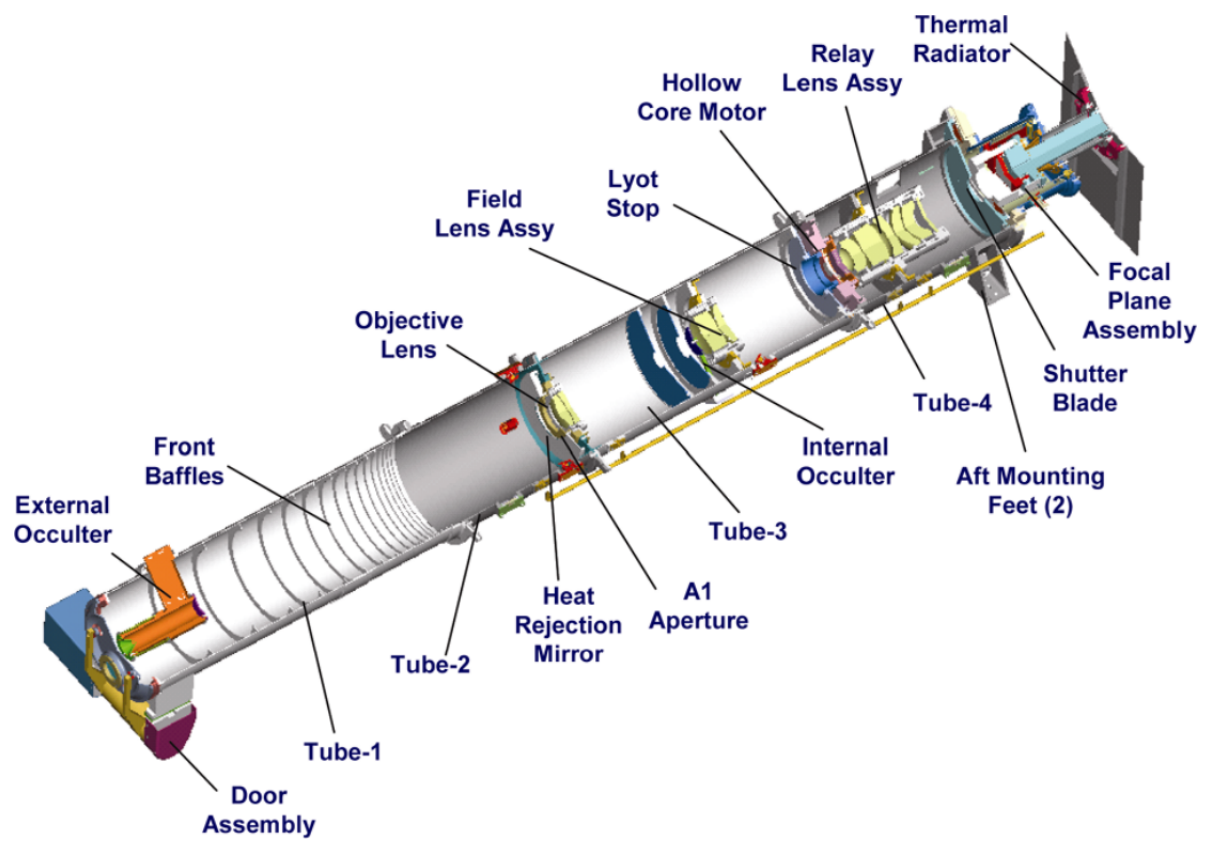

Fig. 5.5: Schematic of the LASCO/COR 2 instrument with the external occulter diffraction design. (Howard et al., 2008b)

and COR B is given as $1.35 \times 10^{-12}$ and $1.25 \times 10^{-12} B_{\odot} / \mathrm{DN}$, where DN is the Data Numbers per CCD pixel and the mean solar photospheric brightness $B_{\odot}$ is $2.3 \times 107 \mathrm{Wm}^{-2} \mathrm{sr}^{-1}$ The coronagraphs have an exposure times below 4 seconds. A summary of the instrument properties of the previously presented coronagraphs is given Table 5.1.

The Heliospheric Imager (HI) is part of the SECCHI suite. Its design consists of a wide-angle visible-light imaging system for the detection of coronal mass ejection (CME) events in interplanetary space. The band-pass of HI-1 is chosen to approximately match the one of COR 2. A wide spectral response of the HI 2 camera maximises the weak coronal signal at large solar elongations. Brightness signal sensitivity requirements are therefore based on the need to extract the CME signal from the sources. The lower detection limit for $\mathrm{CME}$ intensities is given as $3 \times 10^{-15} \mathrm{~B}_{\odot}$ for $\mathrm{HI} 1$ and as $3 \times 10^{-16} \mathrm{~B}_{\odot}$ for $\mathrm{HI}-2$. The F-corona and starfield have typical radiances in the order of a few $10^{-14} \mathrm{~B}_{\odot}$ at 45 degrees from the Sun, so identifying and tracking a CME requires relative photometry and signal separation with a precision of $10^{-2}$. The HI-1 and HI-2 telescope boresights are mounted 
Table 5.1: Summary of coronagraph properties equipped at SOHO and STEREO.

\begin{tabular}{|c|c|r|c|c|c|}
\hline Mission & Instrument & \multicolumn{1}{c|}{ FOV } & Image Size & Cadence & Polariser \\
\hline \hline \multirow{3}{*}{ SOHO } & LASCO C1 & $1.1-3.0 \mathrm{R}_{\odot}$ & $1024 \times 1024$ & $30 \mathrm{~min}$ & $0^{\circ} ; \pm 60^{\circ}$ at $400-656 \mathrm{~nm}$ \\
& LASCO C2 & $1.5-6.0 \mathrm{R}_{\odot}$ & $1024 \times 1024$ & $30 \mathrm{~min}$ & $0^{\circ} ; \pm 60^{\circ}$ at $400-835 \mathrm{~nm}$ \\
& LASCO C3 & $3.7-30.0 \mathrm{R}_{\odot}$ & $1024 \times 1024$ & $30 \mathrm{~min}$ & $0^{\circ} ; \pm 60^{\circ}$ at $400-1050 \mathrm{~nm}$ \\
\hline \multirow{2}{*}{ STEREO } & COR1 & $1.4-4.0 \mathrm{R}_{\odot}$ & $1024 \times 1024$ & $5 \mathrm{~min}$ & $0^{\circ} ; \pm 60^{\circ}$ at $\mathrm{H}_{\alpha}(656.3 \mathrm{~nm})$ \\
& COR2 & $2.5-15.0 \mathrm{R}_{\odot}$ & $2048 \times 2048$ & $15 \mathrm{~min}$ & $0^{\circ} ; \pm 60^{\circ}$ at white-light \\
\hline \hline
\end{tabular}

at angles of $13^{\circ}$ and $53^{\circ}$ away from the principal axis of the instrument which is directed towards the Sun (see Figure 5.7). The opening angles of $20^{\circ}$ and $70^{\circ}$ (see Figure 5.6) allow observing the heliosphere from 15 to $84 \mathrm{R}_{\odot}$ and 66 to $318 \mathrm{R}_{\odot}$, respectively. Thus, they enable the tracking of CME signatures from close to the Sun all the way to Earth.

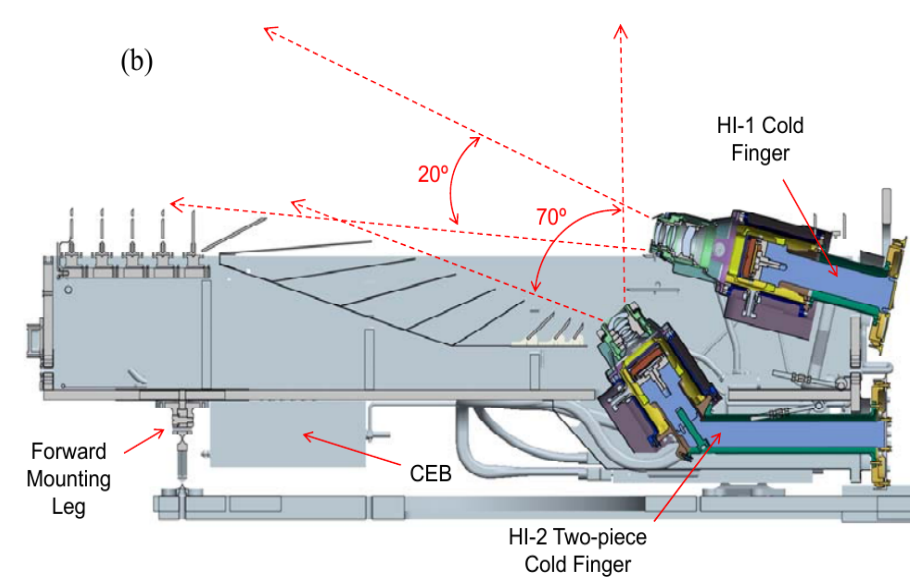

Fig. 5.6: Cross-sectional view through the HI instrument. The angular observation range and the relative orientation of both telescopes are shown. (Eyles et al., 2009)

The respective field of views for the STEREO Coronagraphs and Heliospheric Imagers are presented in Fig 5.7 and Fig 5.8. In the following, the interplay between the HI 1 instrument and the STEREO and LASCO coronagraphs is demonstrated via the CME event on January 7, 2014. An overview of the STEREO satellite configuration on the respective date, the HI FOVs and the two-dimensional CME shape projection is shown in Figure (5.9). On this date the satellites were positioned in the ecliptic at angles of about $150^{\circ}$ (STEREO A) and $-153^{\circ}$ (STEREO B) with respect to Earth (SOHO). The shown CME $(\sim 2500 \mathrm{~km} / \mathrm{s})$ propagated in the opposite direction to STEREO B, at a heliocentric longitude of 38 degrees and with a half width of 64 degrees (Mays et al., 2015). Thus, it manifested as a backside halo event in COR 2B and as a partial Halo in COR 2A and SOHO C3. At around 19:40 UT the event started to exceed the FOV of COR 2A (see Figure 5.10). The respective CME front could then be detected in HI-1A (Figure 5.11) at around 20:10 UT. At this point in time the event was not yet observable in HI-1B. The visible white light features in HI-1B originated from coronal streamers. 


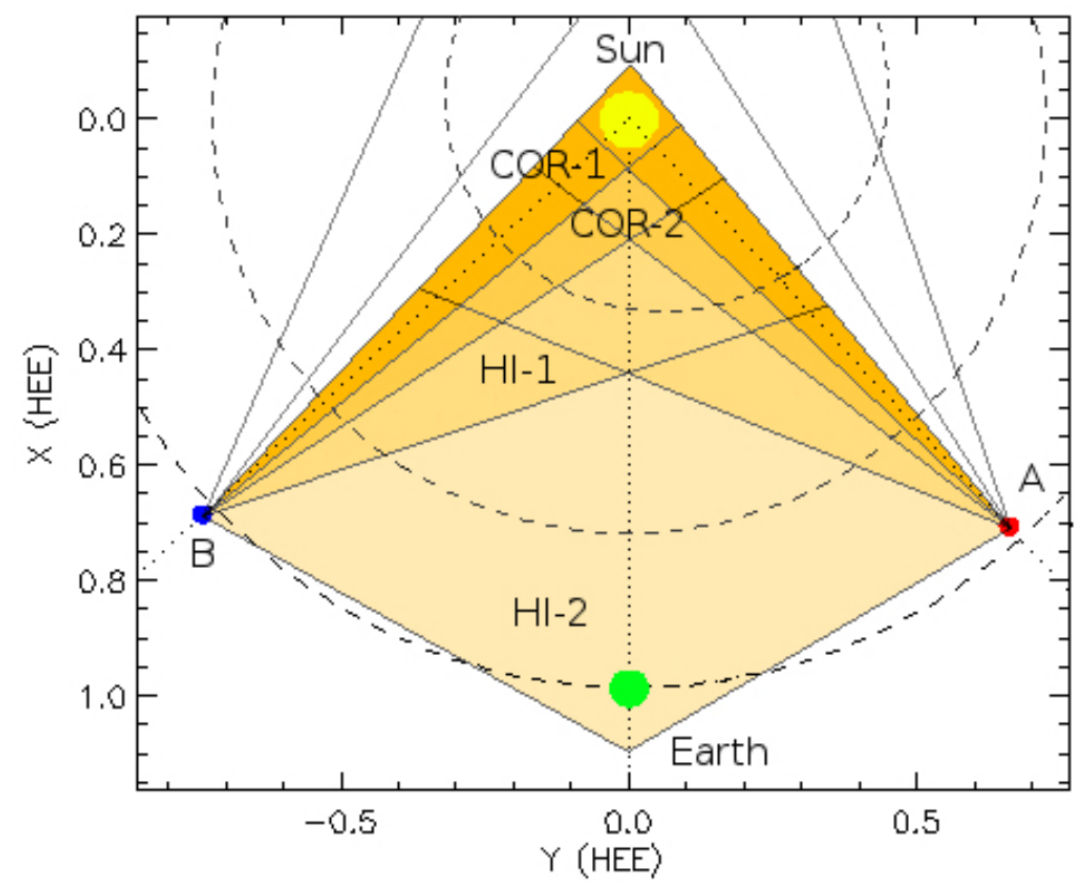

Fig. 5.7: Field of view of the different STEREO/SECCHI telescopes shown for the satellite positions on March 2009. (Adapted from STEREO Science Center)

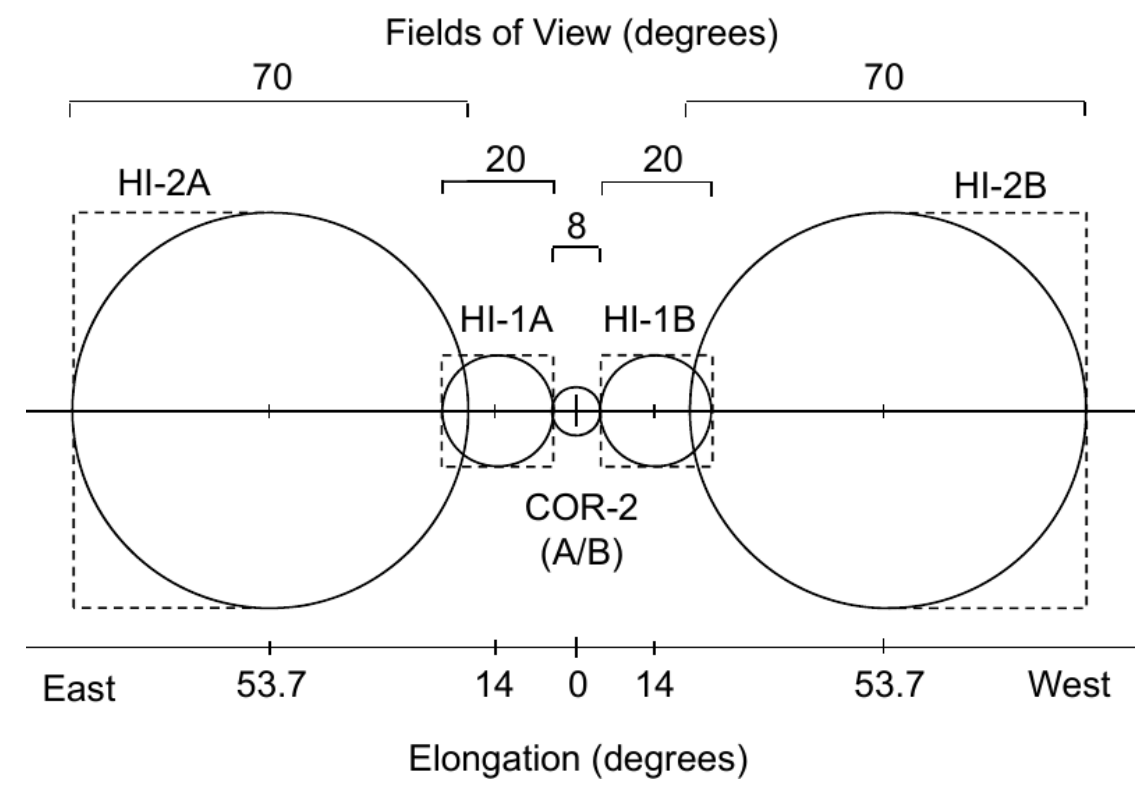

Fig. 5.8: Schematic of the field of view configuration of the different SECCHI instrument. The Sun-centered coronagraphs are capable to observe all solar latitudes, whereas the HI instruments can only observe to a maximum latitudinal angle of \pm 35 degrees perpendicular to the ecliptic. (Eyles et al., 2009) 


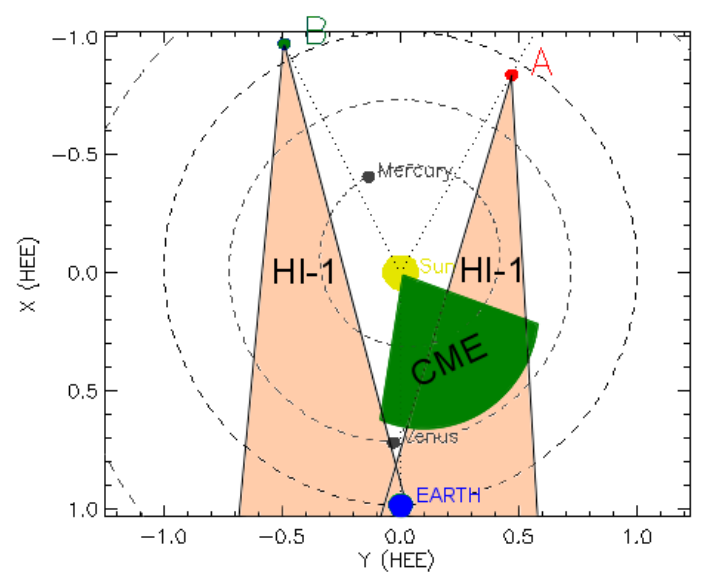

Fig. 5.9: Satellite positions of STEREO A, STEREO B and SOHO (Earth) and the FOV of HI-1 on January 7, 2014. Due to the CME propagation direction and width, the event will have crossed the whole FOV of HI-1A before it even becomes visible in HI-1B. (Adapted from STEREO Science Center)

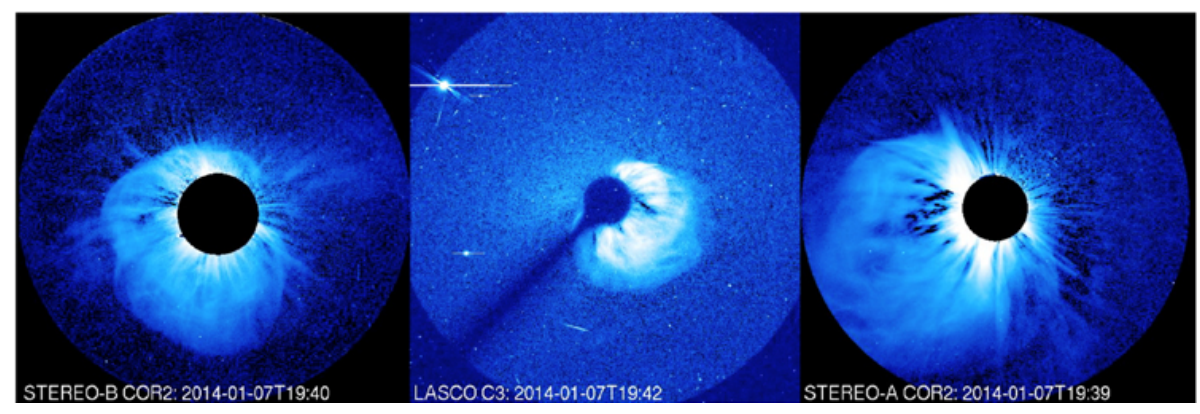

Fig. 5.10: COR 2B, LASCO C3 and COR 2A observations on January 7, 2014 around 19.40 UT. (Mays et al., 2015)
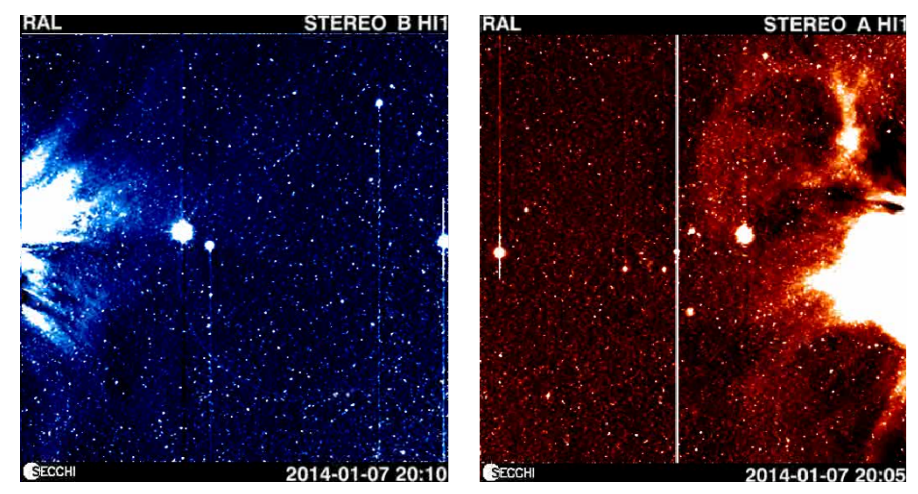

Fig. 5.11: STEREO/HI-1 observation on January 7, 2014. Around 20.00 UT a close CME front is visible in HI-1A. At this point in time, the event is not visible in HI-1B, which only shows some open coronal streamer signatures. (Rutherford Appleton Laboratory) 
The HI instruments permitted many spectacular discoveries as the impact of a CME on Venus (Rouillard et al., 2009), the faint optical emission of Co-rotating Interaction Regions (CIRs) (Rouillard et al., 2008) or the CME induced disconnection of a comet tail (Vourlidas et al., 2007), to name just a few.

\subsubsection{Heliospheric CME Tracking}

In this thesis heliospheric tracking will play a role in the event selection criteria of CME events. Coronagraph and HI instruments are of special interest when it comes to the analyses of CME velocity evolutions. For this purpose, Sheeley et al. (1999a) developed J-maps, a technique for the tracking of solar transients using time-height maps of observations from LASCO/SOHO coronagraphs. Vertical radial stripes of CME fronts or CME shock fronts are extracted at the fixed position angle (PA). The position angle is defined as the angle of the main CME expansion direction as it appears in the POS projection of the observing instrument. From a sequence of this coronagraph images slices, a CME (shock) front signature appears as positively inclined line. The inclination depends on the speed and direction of the transient in relation to the observing instrument. Sheeley et al. (2008) and Rouillard et al. (2008) extended the j-maps technique to HI observations, which enabled tracking to much further distances (see Figure 5.12).

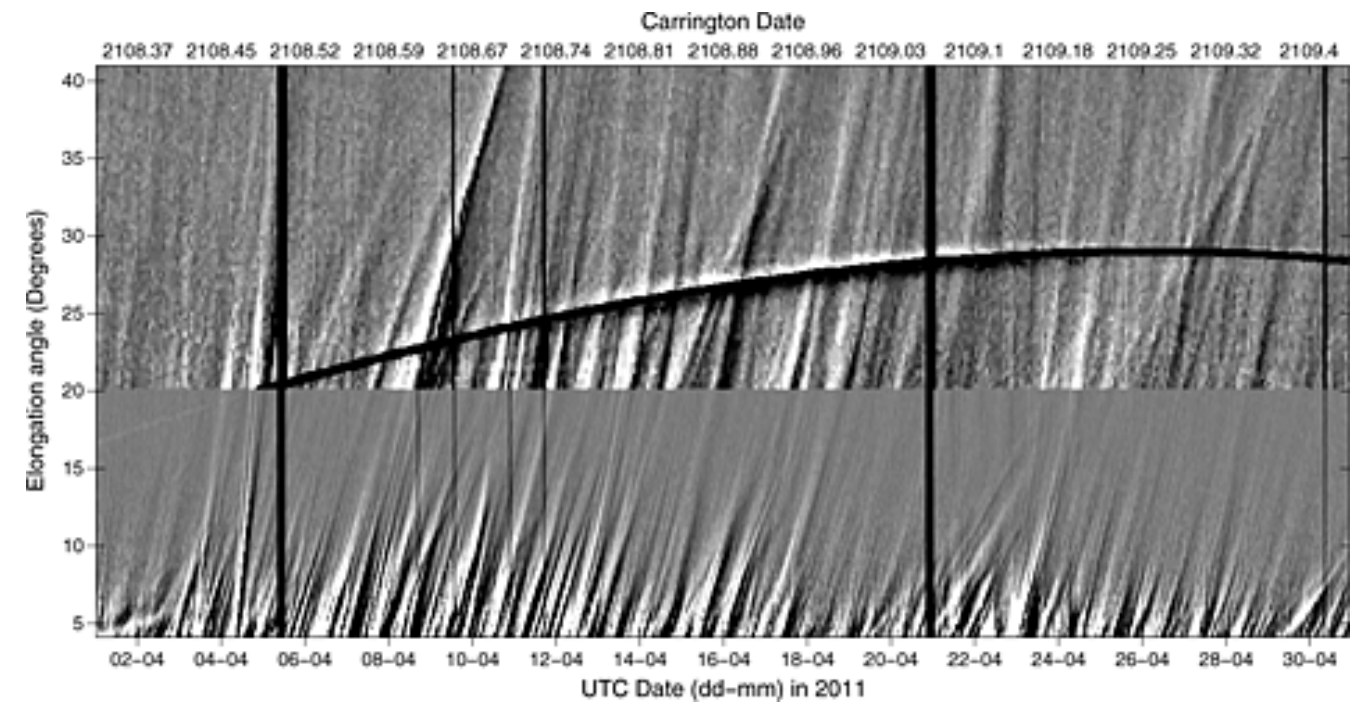

Fig. 5.12: Time-elongation map (j-map) constructed from vertical stripes of STEREO-A difference images along the PA corresponding to the ecliptic. The HI-1 FOV is plotted to $20^{\circ}$ and from thereon continued with the HI-2 FOV. The curved track is the signature of Venus. Solar transients in the form of shocks and CME fronts are visible as white and black lines with positive gradients. The vertical black bars are data gaps. (Tucker-Hood et al., 2015)

\subsection{Advanced Composition Explorer (ACE)}

At this point the Advanced Composition Explorer mission is mentioned as it plays an important role in the analysis of CME arrivals at Earth. The ACE mission is a NASA program to explore and study the elemental and isotopic composition of the solar wind, interplanetary medium, the local interstellar medium and galactic matter. The spacecraft was launched on August 25, 1997. On December 12, 1997 it reached the Lagrangian liberation point (L1) on the Sun-Earth line, at around 1.5 million km distance from Earth 


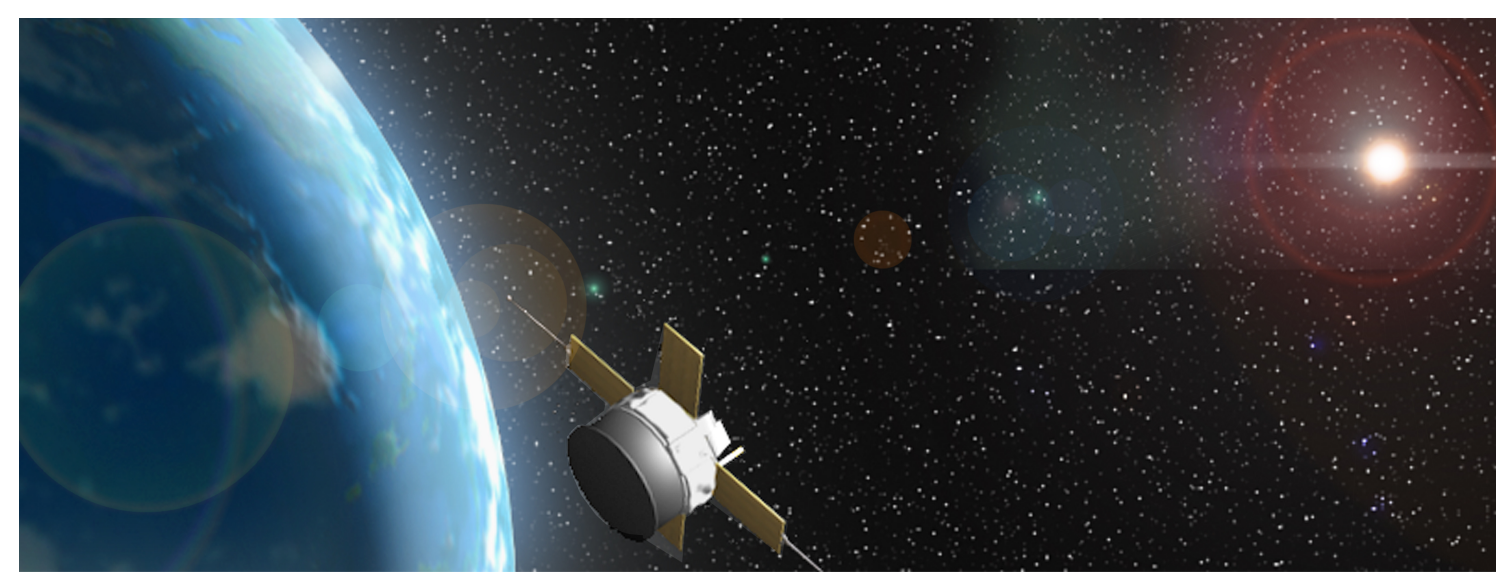

Fig. 5.13: Artistic image of the ACE spacecraft. (Thomas Zurbuchen - Advanced Composition Explorer website)

(see Fig 5.13). The mission goal is to investigate the origin and evolution of solar and galactic matter, the formation of the solar corona and the acceleration of the solar wind. This also covers the in-situ analysis of CMEs, CIRs and solar energetic particles embedded in the ambient solar wind. ACE provides near-real-time data with a continuous coverage of solar wind parameters and solar energetic particle intensities. This data is used by the NOAA Space Weather Prediction Center to enhance the forecast of solar storms. The most important data for CME arrivals is gathered from the following two ACE instruments:

The Solar Wind Electron Proton Alpha Monitor (SWEPAM) (McComas et al., 1998) experiment provides the bulk solar wind observations. It consists of two independent instruments, one for the detection of ions and one for electrons. Both instruments employ a spherical electrostatic analyser whose electric field only allows the passage of ions or electrons with a specific energy. They provide a 64 -second resolution of the full electron and ion distributions. Subsets of these observations are provided by real-time telemetry, to measure the elemental and isotopic composition of the solar wind and examine solar phenomena, such as CMEs or interplanetary shocks.

The Magnetic Field Monitor (MFM) (Smith et al., 1998) provides continuous measurements of the local magnetic field in the interplanetary medium. MFM measurements are fundamental for the interpretation of the energetic and thermal particle distribution, which is measured simultaneously by ACE. The MFM consists of a pair of triaxial fluxgate sensors mounted on opposing solar panels and produces continuous real time data of the interplanetary magnetic field with 1-second resolution. These data are provided to the Space Environmental Center (SEC) of the National Oceanographic and Atmospheric Association (NOAA) for world-wide dissemination.

\subsection{Coordinate Systems and Transformations}

This thesis will refer to some heliographic and heliocentric coordinate systems, which are presented in the following. 


\subsubsection{Heliographic Coordinates Systems}

Two heliographic coordinate systems, namely Stonyhurst and Carrington, are used in this work for the analysis of CME events. These and further solar coordinate systems are summarised in Thompson (2006). The positions in heliographic coordinate system are expressed via latitude $\Theta$, longitude $\Phi$ and radial distance from the solar centre $r$. All coordinates can also be related to a position on the solar surface by setting the radial distance to $r=1 R_{\odot}$.

\section{Stonyhurst Heliographic Coordinates}

The Stonyhurst system is seen from Earth and defined with the $\mathrm{X}$-axis in the direction of the solar equator, the $\mathrm{Y}$-axis in the direction of the central median and the Z-axis along the North Pole of the solar rotation. The Sun rotates synodically underneath this fixed coordinate system. It is generally represented with polar coordinates (see Figure 5.14). The Stonyhurst angles $\Theta$ and $\Phi$ originate from the solar visible disk, as seen from Earth, and increase towards the solar North Pole and the western limb, respectively.

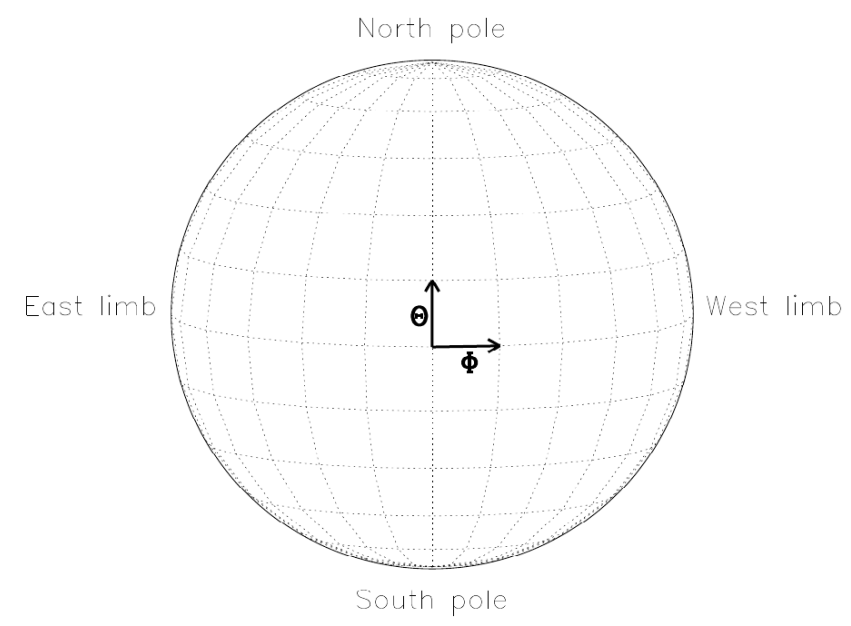

Fig. 5.14: Stonyhurst Coordinate systems. (Thompson, 2006)

\section{Carrington Heliographic Coordinates}

In contrast to the Stonyhurst system, the Carrington system is not fixed to Earth but rotates along with solar surface. A full solar rotation takes 25.38 days which transforms to 27.28 days in mean synodic period (Stix, 1989). Each rotation is sequentially numbered, starting with the first Carrington rotation on November 9, 1853. The latitude $\Theta$ is identical in the Stonyhurst and Carrington coordinate system whereas the Carrington longitude $\Phi_{\mathrm{C}}$ has an offset from the Stonyhurst longitude $\Phi$ with a time-dependent scalar value:

$$
\Phi_{\mathrm{C}}=\Phi+L_{0}
$$

where $L_{0}$ is the Carrington longitude of the central meridian as seen from Earth. At the beginning of each Carrington rotation, the Carrington longitude has the value of $360^{\circ}$ and steadily decreases towards $0^{\circ}$. 


\subsubsection{Heliocentric Coordinate Systems}

In heliocentric coordinate systems, the true spatial position of solar feature is given in relation to the center of the Sun. These right-handed coordinate systems consist of three mutually perpendicular axes, so that it is sufficient to only define two axes. The two heliocentric coordinate systems used throughout this work are presented in the following.

\section{Heliocentric Earth Equatorial (HEEQ)}

In the Heliocentric Earth Equatorial system, the X-axis is positioned along the Sun-Earth line and the Z-axis along the North Pole of the solar rotation axis. The HEEQ System is closely related to Stonyhurst by the following spherical coordinate transformation:

$$
\begin{aligned}
X_{\mathrm{HEEQ}} & =r \cos \Theta \cos \Phi \\
Y_{\mathrm{HEEQ}} & =r \cos \Theta \sin \Phi \\
Z_{\mathrm{HEEQ}} & =r \sin \Theta
\end{aligned}
$$

and the inverse transformation

$$
\begin{aligned}
& r \sqrt{X_{\mathrm{HEEQ}}^{2}+Y_{\mathrm{HEEQ}}^{2}+Z_{\mathrm{HEEQ}}^{2}} \\
& \Theta=\tan ^{-1}\left(Z_{\mathrm{HEEQ}} / \sqrt{X_{\mathrm{HEEQ}}^{2}+Y_{\mathrm{HEEQ}}^{2}}\right) \\
& \Phi=\arctan 2\left(X_{\mathrm{HEEQ}}, Y_{\mathrm{HEEQ}}\right) .
\end{aligned}
$$

\section{Heliocentric Earth Ecliptic (HEE)}

In the Heliocentric Earth Ecliptic system, the $\mathrm{X}$ axis points along the Sun-Earth line and the Z-axis points to the ecliptic North Pole. This system is often preferred in order to predict the arrival of CMEs at other planets or satellites. In this work it is used to display STEREO satellite positions as well as the CME orientation in the heliosphere. The HEE coordinates can be transformed to HEEQ coordinates via two transformation matrices (Hapgood, 1992):

$$
S_{1}=<\Phi_{\odot}+180^{\circ}, Z>\text { and } S_{2}=<\theta_{\odot}, Z>*<i, X>*<\omega, Z>,
$$

where $\lambda_{\odot}$ is the solar ecliptic longitude, $\theta_{\odot}$ is the longitude of the solar central meridian, $i$ is the inclination of the solar equator and $\omega$ is the ecliptic longitude of the ascending node of the solar equator. The transformation of HEEQ to HEE can be performed with the transformation matrix $S_{1} S_{2}^{-1}$. 


\section{Thomson Scattering}

CME masses are determined based on their total brightness in coronagraph emissions. The white-light brightness of CMEs depends on both the local electron density and the efficiency of the Thomson-scattering process. In this section, the Thomson scattering mechanism and its application to the solar corona is discussed. It is further shown that, despite projection effects, the observed projected brightness of CMEs is sufficient to obtain solid CME mass estimations.

The Thomson scattering theory goes back to Sir Joseph John Thomson (1903) and describes the elastic scattering of a photon by free charged particles. Thomson scattering is used in plasma diagnostics to measure plasma densities as well as temperatures and finds application in fusion reactors (Woskoboinikow et al., 1983). The frequency and the kinetic energy of the photon is preserved during the scattering process. It is implicit that the coherence length of the radiation is small compared to the separation of the particles which would otherwise require the theory of coherent scattering. An incident electric field wave provokes a charged particle electron to oscillate along its radial electric field component. This causes the emission of dipole radiation in the frequency of the incident electric field. The strongest intensity $I=\sin ^{2}(\chi)$ occurs at the direction perpendicular $(\chi=90)$ to incident electric field. The intensity of scattered light from a point $\mathrm{P}$ depends linearly on the total number of scattering electrons at that point. Hence, if the intensity of scattered light is known, the total number of electrons contributing to this intensity can be calculated. This allows the calculation of the total electron content of a CME and therefore the total CME mass. However, the intensity calculation is very sensitive to $\chi$.

In Figure (6.1) an incident electric field wave (e.g. photon) excites an electron to oscillate along its radial electric field component. This causes the emission of dipole radiation in the frequency of the incident electric field. The strongest intensity $I=\sin ^{2} \chi$ occurs at the direction perpendicular $\left(\chi=90^{\circ}\right)$ to the incident electric field. For $\chi=90^{\circ}$ this radiation is furthermore completely polarised.

\subsection{Thomson Scattering in the Corona}

The formalisation of the Thomson scattering theory for an electron in the solar atmosphere was first carried out by Schuster (1879) and Minnaert (1930). The radiation from the solar photosphere is completely unpolarised. Therefore all coronal electrons oscillate with the same amplitude in all directions perpendicular to the incident light. An observer notices that the perpendicular oscillation components have the same amplitude whereas the parallel components are foreshortened, depending on the viewing angle $\chi$. Thus, the radiation may appear unpolarised, partially polarised or completely linearly polarised (see

\footnotetext{
${ }^{1}$ Source: http://en.wikipedia.org/wiki/User:PAR (2004)
} 


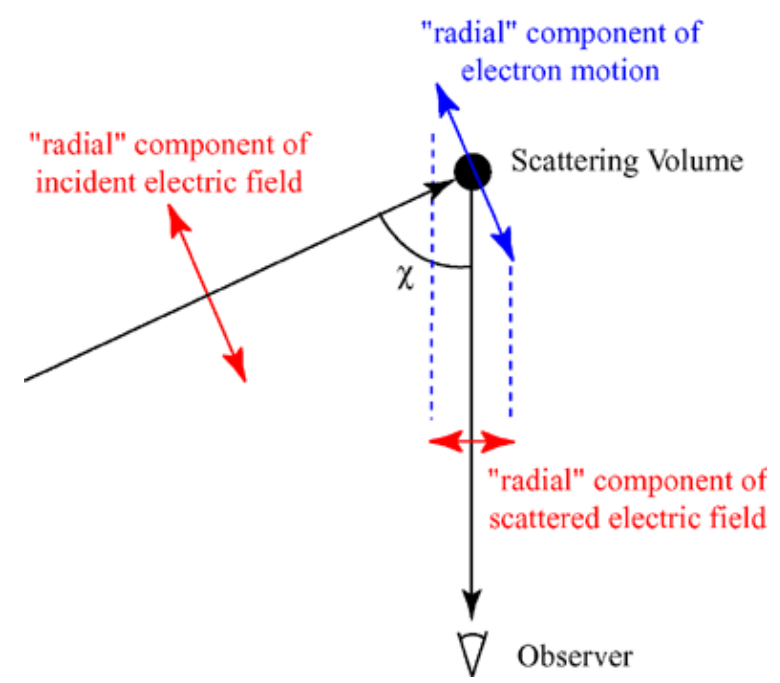

Fig. 6.1: Thomson scattering mechanism. An incident photon stimulates the oscillation of an electron along its radial electric field. Shortly after, the electron emits a dipole radiation towards the observer. ${ }^{1}$

Figure 6.2). The differential scattering cross-section of an electron is given by:

$$
\frac{d \sigma}{d \Omega}=\frac{\text { radiated power } / \text { unit solid angle }}{\text { incident power/unit area }} .
$$

According to Jackson (1975), the differential scattering cross-section for a completely unpolarised incident electric field can be written as:

$$
\frac{d \sigma}{d \Omega}=\left(\frac{e^{2}}{4 \pi \varepsilon_{0} m_{e} c^{2}}\right)^{2} \frac{1}{2}\left(1+\cos ^{2} \chi\right)
$$

where $e$ is the electron charge, $m_{e}$ is the electron mass, $c$ is the speed of light, $\varepsilon_{0}$ is the electric permittivity of free space. The integration over all solid angles yields the Thomson scattering cross-section $\sigma_{T}$ :

$$
\sigma_{T}=\frac{8 \pi}{3}\left(\frac{e^{2}}{4 \pi \varepsilon_{0} m_{e} c^{2}}\right)^{2}=\frac{8 \pi}{3} r_{e}^{2}=6.65 \times 10^{-29} \mathrm{~m}^{2}
$$

Any radiation within the area defined by the classical electron radius $r_{e}$ will be scattered. For the incident electric field one has to consider the decreasing radiation intensity from the solar photosphere towards the solar limb:

$$
I=I_{0}(I-u+u \cos \phi)
$$

where $u$ is the wavelength dependent limb-darkening coefficient and $\phi$ is the angle between the radial emission from a point $\mathrm{S}$ on the solar surface and the straight line between the observer $\mathrm{O}$ and the point $\mathrm{S}$. The tangential $I_{\mathrm{T}}$ and radial $I_{\mathrm{R}}$ components of the scattered radiation from a single electron are given by the expressions:

$$
\begin{aligned}
I_{\mathrm{T}} & =I_{0} \frac{\pi \sigma_{e}}{2 z^{2}}[(1-u) C+u D], \\
T_{\mathrm{P}} & =I_{0} \frac{\pi \sigma_{e}}{2 z^{2}} \sin ^{2} \chi[(1-u) A+u B], \\
I_{\mathrm{R}} & =I_{\mathrm{T}}-I_{\mathrm{P}},
\end{aligned}
$$




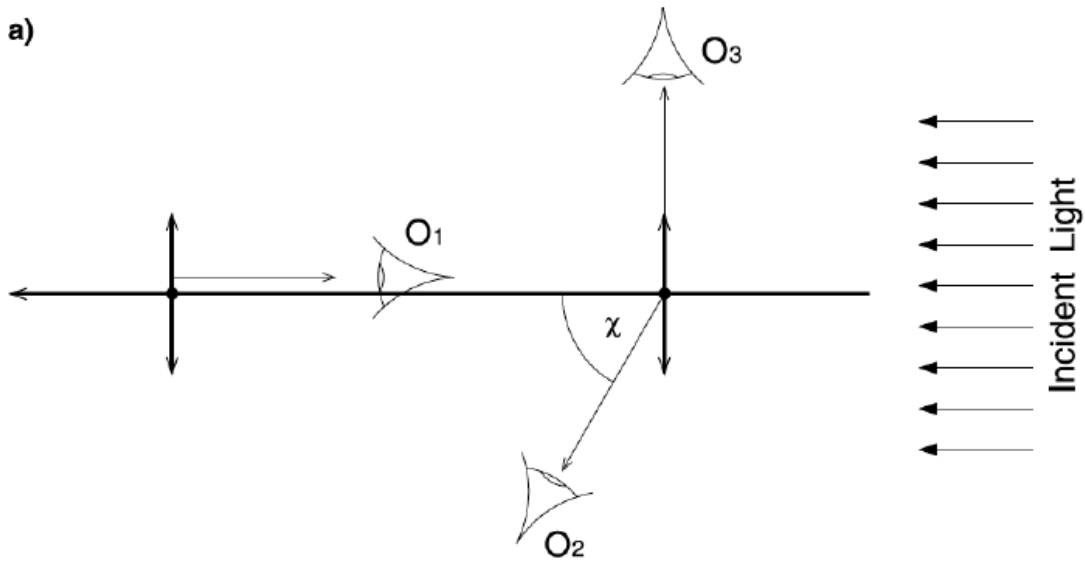

b)

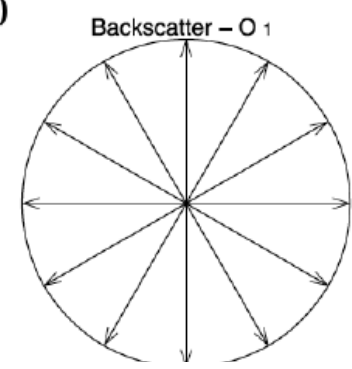

c) From 60 degrees $-\mathrm{O}_{2}$

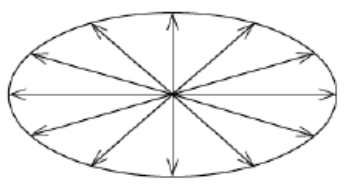

d) From 90 degrees $-\mathrm{O}_{3}$

Fig. 6.2: Schematic of the Thomson scattering mechanism in three dimensions for an incident unpolarised wave. For the observer $\mathrm{O}_{1}$ at $\chi=0^{\circ}$ the emitted light is completely unpolarised. The observer $\mathrm{O}_{2}$ at $\chi=60^{\circ}$ notices a partial polarisation of the light and observer $\mathrm{O}_{3}$ at $\chi=90^{\circ}$ a linear polarisation. (Howard \& Tappin, 2009)

where $I_{0}$ is incident intensity, $\sigma_{e}$ is the electron scattering cross section, $z$ is the distance from scatter to observer, $u$ is a limb darkening coefficient. For a more intuitive derivation $I_{\mathrm{R}}$ can be defined with the help of polarisation intensity $I_{\mathrm{P}}$ (Howard \& Tappin, 2009). $I_{\mathrm{T}}$ and $I_{\mathrm{R}}$ are fully observed at $\chi=0$ degree and $\chi=180$ degree, while $I_{\mathrm{T}}$ is observed at $\chi=90$ degree. Thus far, we have limited the Thomson scattering process to a single scattering electron and considered the photosphere as a point source. For a non-uniform source, it is necessary to integrate the scattered light over the whole solar disk. The finite size of the Sun is incorporated into the coefficients A, B, C, and D defined by the equations (6.8-6.11). These van de Hulst coefficients (van de Hulst, 1950) are given by

$$
\begin{aligned}
& A=\cos \Omega \sin ^{2} \Omega \\
& B=-\frac{1}{8}\left[1-3 \sin ^{2} \Omega-\frac{\cos ^{2} \Omega}{\sin \Omega}\left(1+3 \sin ^{2} \Omega\right) \ln \left(\frac{1+\sin \Omega}{\cos \Omega}\right)\right] \\
& C=\frac{4}{3}-\cos \Omega-\frac{\cos ^{3} \Omega}{3} \\
& D=\frac{1}{8}\left[5+\sin ^{2} \Omega-\frac{\cos ^{2} \Omega}{\sin \Omega}\left(5-\sin ^{2} \Omega\right) \ln \left(\frac{1+\sin \Omega}{\cos \Omega}\right)\right]
\end{aligned}
$$

The total scattered intensity is then:

$$
I_{\text {tot }}=I_{\mathrm{T}}+I_{\mathrm{R}}=2 I_{\mathrm{T}}-I_{\mathrm{P}}
$$

The equations describes light scattered by a single electron at any position in the solar atmosphere (any $\Omega$ ), with the observer at viewing angle $\chi$. The intensity of scattered light 
depends linearly on the total number of scattering electrons $N_{e}$ at that point. From the total intensity, we can therefore directly calculate the total electron content of a CME and therefrom calculate its mass. However, the intensity calculation is very sensitive to $\chi$ so that projection effects have to be considered.

\subsection{Projection Effects}

According to Thomson scattering theory the apparent brightness in white light images is a function of the specific angle $\varepsilon$ between the Line of Sight (LOS) and the Sun-observer line. The emission of an extended structure is optically thin and will therefore contain brightness contributions for all material along any arbitrary LOS. The brightness of a

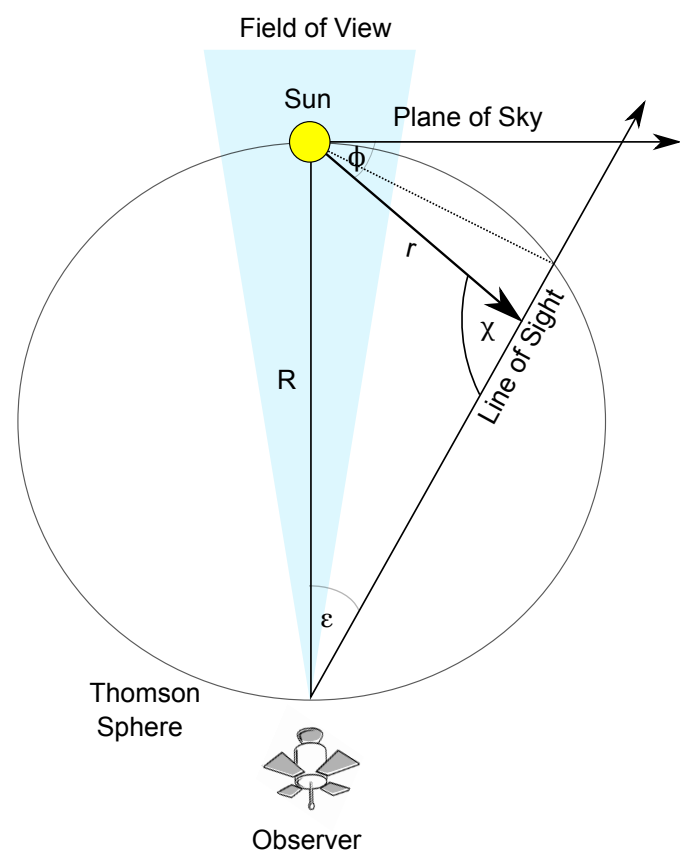

Fig. 6.3: Illustration of the Thomson sphere $(\phi=\varepsilon)$ for an observer with the distance $\mathrm{R}$ to the Sun. (Figure after Vourlidas \& Howard 2006).

single electron $B_{e}(\phi)$ along the LOS contributes most to the overall brightness when the electron is positioned close to the Thomson Sphere. CME white-light observations are projections of the three-dimensional CME structures onto the Plane-of-Sky (POS) and the real 3-dimensional mass distribution is unknown. Hence, the influence of projection effects onto the CME mass determination has to be considered. The POS is overlapping with the Thomson Sphere within the maximal FOV (see Figure 6.4) of typical satellite based coronagraphs (Vourlidas \& Howard, 2006) and is therefore the location of maximum scattering. CMEs erupting close to the POS are better observable whereas large angles away from the POS decrease in scattering efficiency. We see that along any LOS through the corona, the point at $\chi=90^{\circ}$ is the point of minimum distance from the Sun where three effects compete with each other. The scattering efficiency is a minimum, the incident intensity received from the Sun is a maximum and the electron density is a maximum. As the two latter effects have a bigger contribution, the scattered light has a maximum at $\chi=90^{\circ}$.

This leads to the first assumption, that the brightness analysis can be made relative to the POS as location of maximum scattering. In other words, all CMEs are considered 
to propagate along the POS $\phi=0^{\circ}$. The second approximation suggests that all CMEs are confined to a 2-dimensional plane which is directed perpendicular to the ecliptic and towards the propagation direction of the CME. The consequences of both approximations were studied by Vourlidas et al. (2010). They showed that the first assumption leads to a mass underestimation by a factor of two and more for SOHO mass calculations when the actual propagation angle away from the POS is $\phi \geq 60^{\circ}$ (see Figure 6.5). In the other case, when the CME brightness is scaled with the actual propagation direction, the CME mass results will be more accurate for $\phi \leq 60^{\circ}$ but then will become increasingly overestimated. We use this approach in this thesis as we want to investigate upper mass limits and overestimations by other coronal features. Based on the premise that the CME has an angular width of $60^{\circ}$. Vourlidas et al. (2010) showed that the second assumption only underestimates the CME mass by $15 \%$.

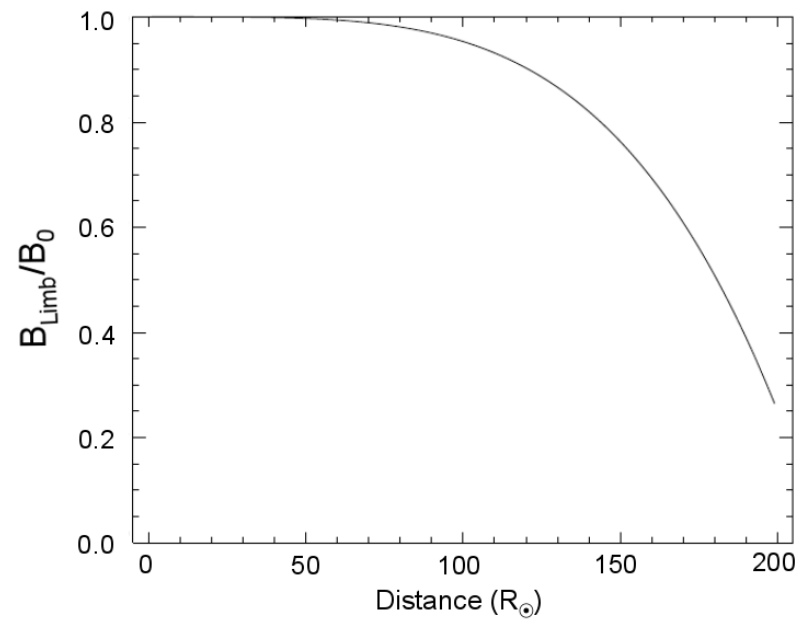

Fig. 6.4: Range of validity of the POS assumption currently used for CME brightness calculations. The ratio $B_{\mathrm{limb}} / B_{0}$ is the ratio of the calculated brightness of an electron $B_{0}$ positioned on the Thomson shere relative to its brightness $B_{\text {limb }}$ at the POS. (Figure adapted from Vourlidas et al. 2010)

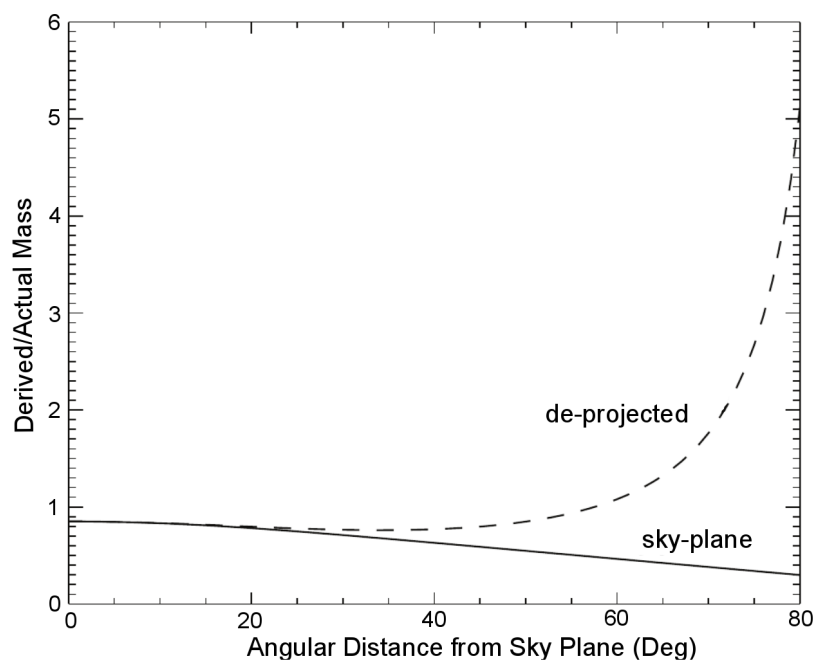

Fig. 6.5: Comparison between the derived and actual CME mass by assuming the electron is either directed along the POS (sky-plane) or along the actual main propagation direction $\phi$ away from the POS (de-projected). This estimation is based on a CME with an angular width of $60^{\circ}$ at $5 R_{\odot}$. (Figure adapted from Vourlidas et al. 2010) 



\section{Geometrical CME Modelling}

CMEs are 3-dimensional objects but their morphology can only be observed as 2-dimensional projections in coronagraph images. Further, their propagation can only be measured in the observer's POS. Thus, the observed CME morphologies and velocities are subject to projection effects. These projection effects are discussed with the help of a very simplistic cone shape geometrical model and compared for the cases that a CME propagates towards the observer and that it propagates along the POS of the observer. It will be discussed, that some CME properties can not be derived from a single observation point but need at least stereoscopic observation with a sufficient separation of the observers. In the next step, a more physically based model, the Graduated Cylindrical Shell (GCS) model is introduced and applied to ten stereoscopically observed CME events.

Some illustrative examples for limb, partial halo and halo appearances of CME have been presented in Chapter 4. It was mentioned that the different projection shapes in coronagraph images mostly result from the propagation direction of the CME to the observer. In particular, CMEs can appear as cone-like shaped events if they propagate along the POS of the observer (limb-event) and as circular shaped if they propagate towards or away from the observer (halo-event, disk-event). In cases where only the flank of a CME is directed towards or away from the observer, they posses a more elliptical shape (partial-Halo event). In a pure geometrical interpretation such an appearance can in first order be associated with a 3-dimensional cone-like morphology. Projection effects for such a geometry have been frequently studied in the past. In Figure 7.1 it is shown, that the CME opening angle and the CME height are well observable if the CME is seen as limb event but not if it is seen as Halo.

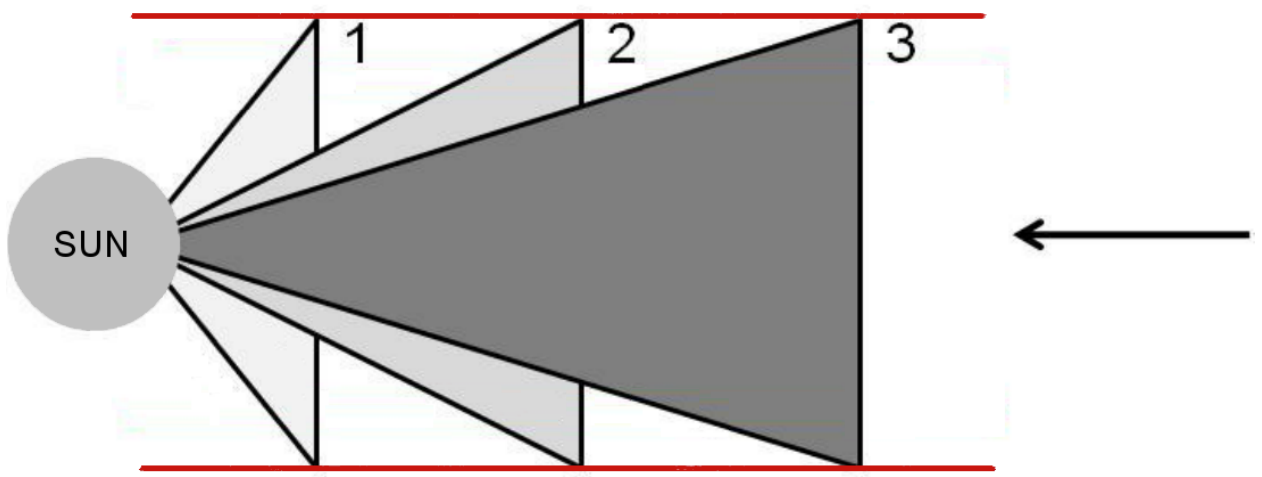

Fig. 7.1: Three cones directed toward the observer represent different CMEs which are seen as halo CMEs for the observer and as limb CMEs for the reader. The outer edge of circular shape is marked with the horizontal lines. The observer sees all three CMEs as halo projections with the same circular shape. In the view perpendicular to the paper, the CMEs appear as limb CMEs and their opening angle and height are well observable. (Adapted from Gopalswamy 2010) 
Even with geometrical modelling the heights and the cone angles of halo CMEs are not accessible for measurements. Therefore, Burkepile et al. (2004) concluded that the measurement of CME speed and angular width through single spacecraft observations are strongly manipulated by projection effects. Generally, these projection effect should be larger for narrow CMEs. Investigations were made to determine a statistical relationship between the POS speeds of CMEs and the direction of their propagation in the intention to obtain a true speed of CME (dal Lago et al., 2002). Also Schwenn et al. (2005) have proposed that the radial speed $v_{\text {rad }}$ can be derived from the expansion speed $v_{\text {exp }}$ of the halo via the empirical relation $v_{\text {rad }}=0.88 v_{\text {exp }}$.

\subsection{Graduated Cylindrical Shell Model}

Geometrical modelling can help to diminish projection effects. In this thesis geometrical modelling with the Graduated Cylindrical Shell (GCS) model is used to derive the CME morphology, kinematics and orientation from coronagraph images. Based on the observations of Cremades \& Bothmer (2004) on the 3-dimensional configurations of CMEs, Thernisien, Howard and Vourlidas (2006) developed the GCS Model to reproduce the large scale 3-dimensional topology of flux rope-like CMEs. Since then the GCS model has been applied to stereoscopic observations of FR-like CMEs by many authors (e.g. Thernisien et al. 2009, 2011; Rouillard 2011).
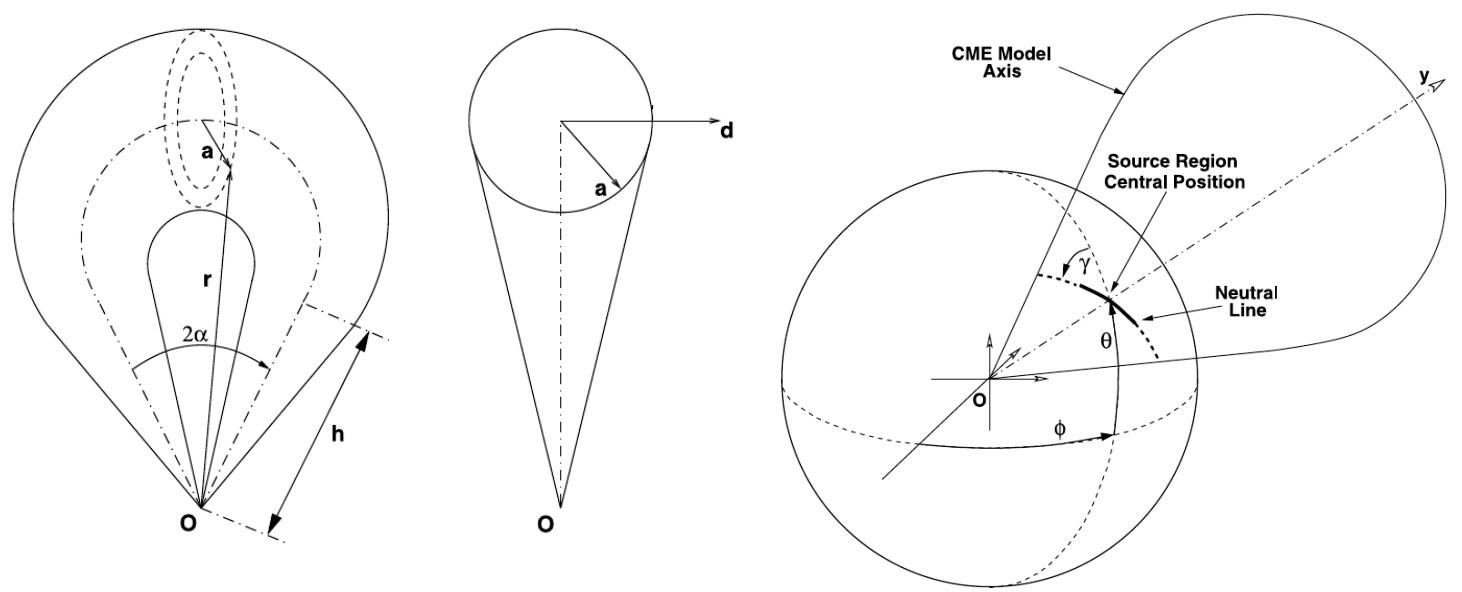

Fig. 7.2: Diagram of the Graduated Cylindrical Shell shape with (left) the "face on view", (middle) the "edge on view" and (right) the orientation of the model on the Sun. (Thernisien et al., 2006)

Table 7.1: Generic GCS morphology parameters.

\begin{tabular}{lcl}
\hline \hline morphology & unity & description \\
\hline$\alpha$ & {$[\mathrm{deg}]$} & angular half width between both legs \\
$h$ & {$\left[R_{\odot}\right]$} & height of the legs \\
$\kappa$ & []$]$ & aspect ratio \\
\hline position & unity & description \\
\hline$\phi, \sigma$ & {$[\mathrm{deg}]$} & Carrington longitude and heliographic latitude of the SR \\
$\gamma$ & {$[\mathrm{deg}]$} & tilt angle of the SR neutral line \\
\hline \hline
\end{tabular}


The basic GCS geometry (see Fig. 7.2) consists of two cone shaped legs, separated by twice the half angle $\alpha$ with the height $h$ and it's origin in the solar centre. Both are on top connected to a tube shaped section with diminishing radius towards the legs. The circular annulus a in the middle of the tube section is given by $a(r)=\kappa r$ where $r$ is the distance from the solar centre to a point of the outer edge of the GCS shape and $\kappa$ is the aspect ratio. Instead of the leg height $h$ it is common to use the parameter for the apex height $h_{\text {apex }}$ derived from Equation (7.1).

$$
h_{\mathrm{apex}}=h \frac{1+\kappa}{1-\kappa^{2}} \frac{1+\sin \alpha}{\cos \alpha}
$$

The GCS model origins from the solar centre and the orientation of the shape is given by the longitude $\phi$, the latitude $\theta$ and, due to its non - rotational symmetry, the tilt angle $\gamma$. The model parameters and their description are summarised in Table 7.1.

\subsection{GCS Modelling Results}

The geometrical modelling of CMEs is somehow subjective. It strongly depends on the corrected interpretation of the CME structures in the coronagraph images. Thus, the quality of the modelling result is also affected by the modeller's experience. For this reason, some modelling experience should be shared at this point. For this purpose, I present 10 GCS fitting samples, each accompanied by a 3D Sun-Earth model in the HEE coordinate system which visualises not only the positions of Sun, Earth and both STEREO satellites but also reflects the shape and orientation of the GCS grid. Based on these 10 examples some of the particularities of CME fitting are discussed afterwards. The events are taken from the HELCATS "KinCat" event catalogue which is further described in Section 8. The GCS model is applied to the full COR2 observation sequence which later will be also used to derive CME speeds. Further, the events from the original list CMEs are fitted at the height of their individual best white-light visibility. It has to be noted, that the brightness scaling of the images does not reflect the actual brightness of the event but is adapted to the best visibility. This approach is helpful to identify the geometrical structures of the events during the geometrical fitting process. For the analysis, it is beneficial to define a reference height. Vourlidas et al. $(2000,2010)$ showed that the majority of CMEs reach a steady-state above about $10 \mathrm{R}_{\odot}$. Sachdeva et al. (2017) showed that CME acceleration largely ends within $5 \mathrm{R}_{\odot}$ for fast events, and between 12 and $50 \mathrm{R}_{\odot}$ for slow events. Therefore, we adopt the height of $12 \mathrm{R}_{\odot}$ as our reference height. For each CME we follow the following modelling procedure:

In the first step, the six GCS parameters are iteratively adapted until the best visual fit of the data is achieved for both STEREO COR2 coronagraphs. Images from the $\mathrm{SOHO} / \mathrm{LASCO}$ coronagraphs, positioned along the Sun-Earth line, are used to confirm the direction of the CME if it is seen as halo in both STEREO satellites. In the next step, the fitting is altered between the first and last COR2 image pairs for a few times. Generally, this approach is helpful to separate the main CME shape from unambiguous parts and shock features, which are both frequently observed in coronagraph white-light images of very intense events (Vourlidas et al., 2013; Vourlidas \& Ontiveros, 2009). In the last step, the CME parameters are adapted to the reference height. For a sequence of mass images the previously modelled GCS shape is applied and expanded self-similarly to the correspondent height. The fitted height values are used for the height-time profile from which the apex speed at $12 \mathrm{R}_{\odot}$ is derived. 


\subsubsection{Sample \#1 - Helcats Event \#84}

Sample event \#1 occurred on 07-09-2011. To this date both STEREO spacecraft have a separation of $162^{\circ}$. The GCS fit of this event is presented in Fig. 7.3 and the fitting parameters in Table 7.2. The orientation of the GCS grid in relation to Earth is presented in Fig. 7.4. The event has a low-intermediate speed $(623 \mathrm{~km} / \mathrm{s})$ and posseses an equally usual intermediate flux rope structure $\left(\kappa=0.42, \alpha=35^{\circ}\right)$. It can be considered as archtype event. The event is observed during the solar maximum, so a lot of streamer activity is present. The typically very black appearing structures are precedent coronal streamers, which were removed by the subtraction of the background corona with an pre-event image.
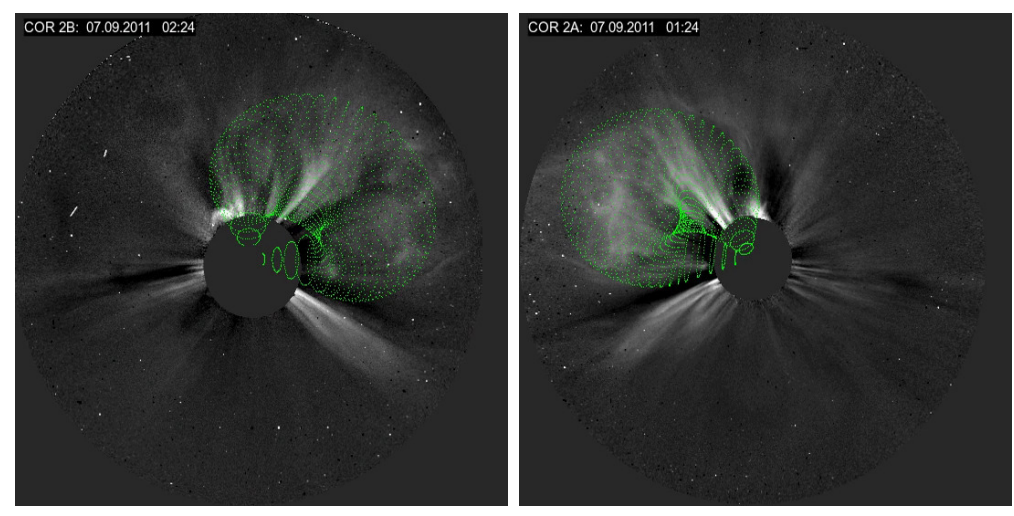

Fig. 7.3: GCS fit of the CME event on 07-09-2011 at 01:07 UT applied to the coronagraph images of STEREO COR2 A(right) and COR2 B(left).

\begin{tabular}{|c|cccccc|}
\hline & $\begin{array}{c}\text { GCS } \\
\text { longitude } \phi\end{array}$ & $\begin{array}{c}\text { GCS } \\
\text { latitude } \theta\end{array}$ & $\begin{array}{c}\text { GCS } \\
\text { asp. ratio } \kappa\end{array}$ & $\begin{array}{c}\text { GCS } \\
\text { half angle } \alpha\end{array}$ & $\begin{array}{c}\text { GCS } \\
\text { tilt } \gamma\end{array}$ & $\begin{array}{c}\text { CME } \\
v_{\text {apex }}\end{array}$ \\
\hline value & $40^{\circ}$ & $34^{\circ}$ & 0.42 & $35^{\circ}$ & $27^{\circ}$ & $623 \mathrm{~km} / \mathrm{s}$ \\
\hline
\end{tabular}

Table 7.2: GCS fitting parameter and determined CME apex speed of the shown event. GCS longitude and latitude are displayed in the HEEQ coordinate system.
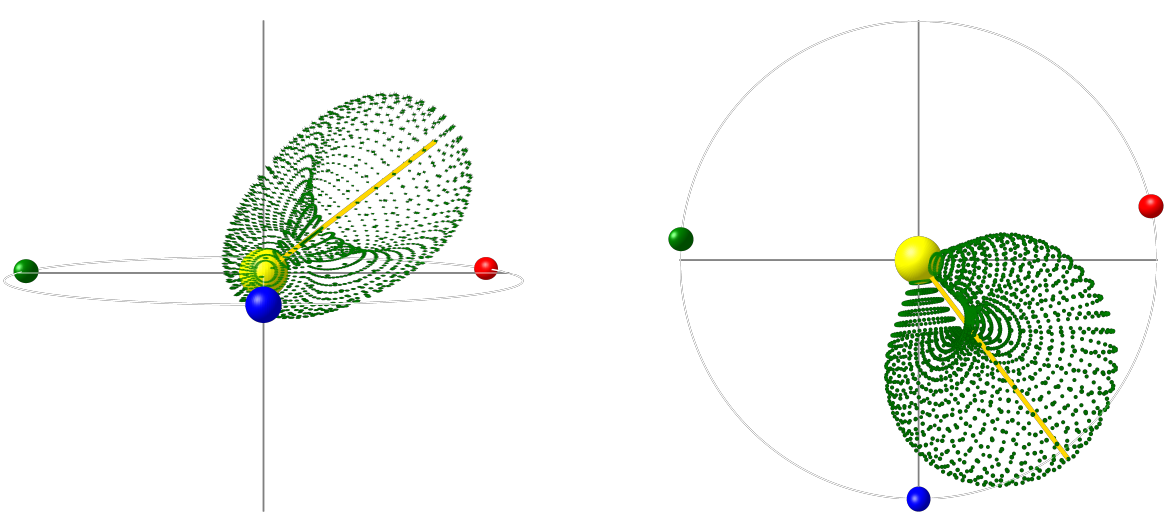

Fig. 7.4: Expansion of 3D GCS grid to an apex height of $1 \mathrm{AU}$ in the HEE coordinate system together with the positions of Earth (blue), STEREO A (red) and STEREO B (green). 


\subsubsection{Sample \#2 - Helcats Event \#122}

Sample event \#2 occurred on 25-10-2013. To this date both STEREO spacecraft have a separation of $70^{\circ}$. The GCS fit of this event is presented in Fig. 7.5 and the fitting parameters in Table 7.3. The orientation of the GCS grid in relation to Earth is presented in Fig. 7.6. The event displays a very common archtype $\left(\kappa=0.45, \alpha=22.5^{\circ}\right)$ of fast CME events $(1088 \mathrm{~km} / \mathrm{s})$ with surrounding shock structure. CME shocks can sometimes contribute to uncertainties in the modelled geometry. However, in this example the event is seen with a sufficient angular separation and the CME brightness is significantly higher as the shock brightness.
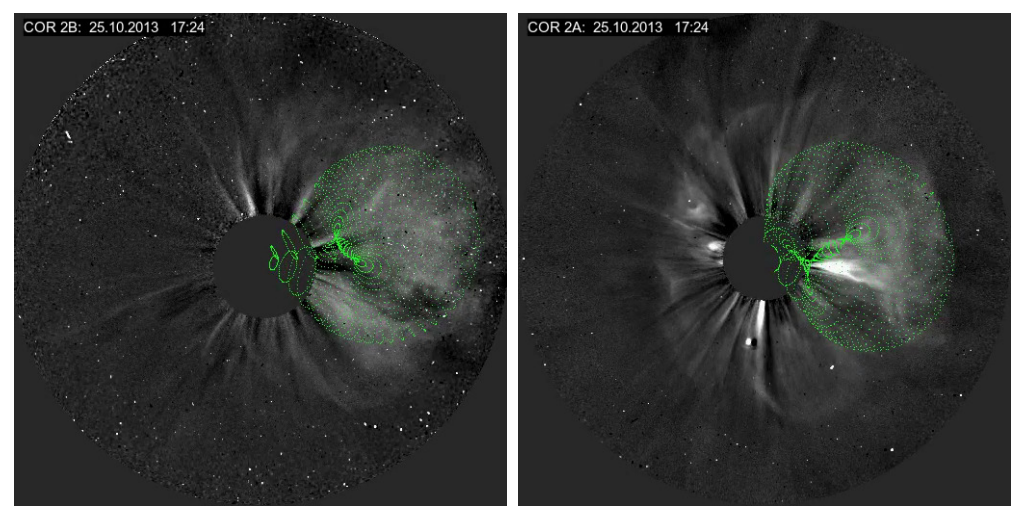

Fig. 7.5: GCS fit of the CME event on 25-10-2013 at 17:24 UT applied to the coronagraph images of STEREO COR2 A(right) and COR2 B(left).

\begin{tabular}{|c|cccccc|}
\hline & $\begin{array}{c}\text { GCS } \\
\text { longitude } \phi\end{array}$ & $\begin{array}{c}\text { GCS } \\
\text { latitude } \theta\end{array}$ & $\begin{array}{c}\text { GCS } \\
\text { asp. ratio } \kappa\end{array}$ & $\begin{array}{c}\text { GCS } \\
\text { half angle } \alpha\end{array}$ & $\begin{array}{c}\text { GCS } \\
\text { tilt } \gamma\end{array}$ & $\begin{array}{c}\text { CME } \\
v_{\text {apex }}\end{array}$ \\
\hline value & $-74^{\circ}$ & $13^{\circ}$ & 0.45 & $22.5^{\circ}$ & $68^{\circ}$ & $1088 \mathrm{~km} / \mathrm{s}$ \\
\hline
\end{tabular}

Table 7.3: GCS fitting parameter and determined CME apex speed of the shown event. GCS longitude and latitude are displayed in the HEEQ coordinate system.
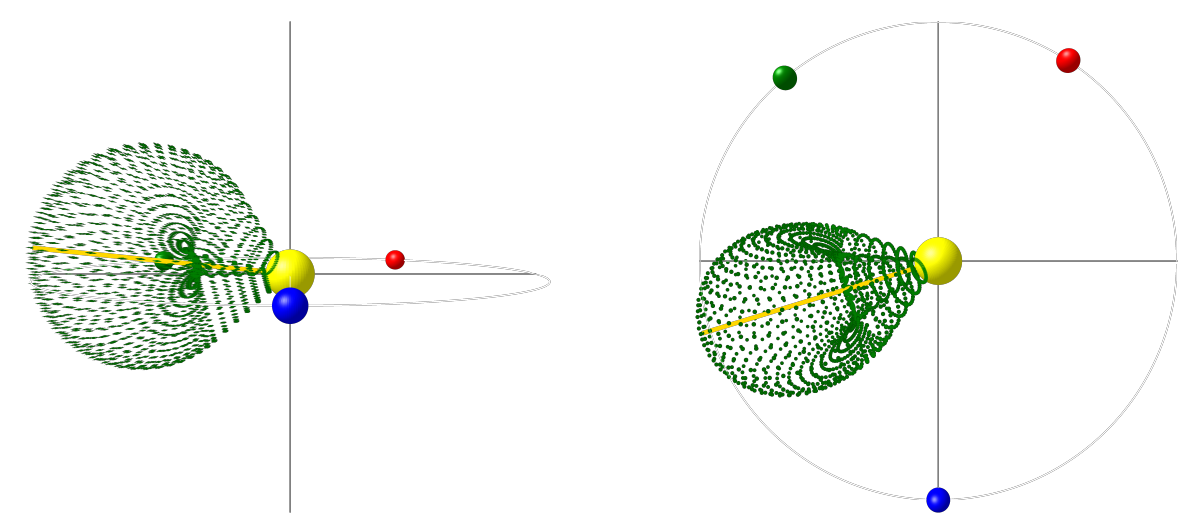

Fig. 7.6: Expansion of 3D GCS grid to an apex height of $1 \mathrm{AU}$ in the HEE coordinate system together with the positions of Earth (blue), STEREO A (red) and STEREO B (green). 


\subsubsection{Sample \#3 - Helcats Event \#2}

Sample event \#3 occurred on 16-05-2007. To this date both STEREO spacecraft have a separation of $8^{\circ}$. The GCS fit of this event is presented in Fig. 7.7 and the fitting parameters in Table 7.4. The orientation of the GCS grid in relation to Earth is presented in Fig. 7.8. The event was observed during the solar minimum with only very few other visible solar activity. During this time period both STEREO spacecraft observed this event with only very little minimal separation $\left(\sigma=8^{\circ}\right)$ which makes both STEREO COR 2 perspectives nearly redundant. The event is seen as limp event so that the determined apex heights $h_{\text {apex }}$ and the resulting apex velocity $v_{\text {apex }}$ is only little effected by projections effects. The half angle $\alpha$, the asp. ratio $\kappa$ and the tilt $\gamma$ are more subject to reservation.
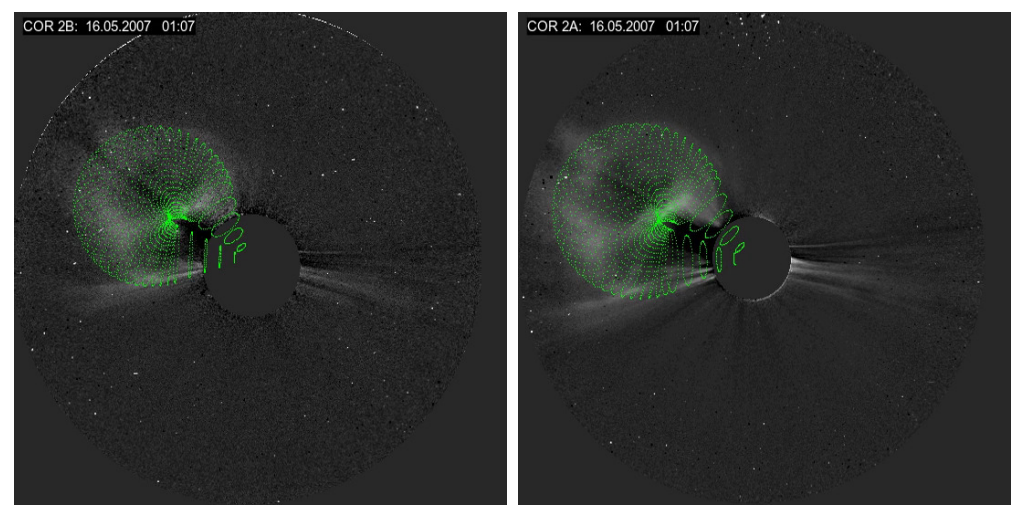

Fig. 7.7: GCS fit of the CME event on 16-05-2007 at 01:07 UT applied to the coronagraph images of STEREO COR2 A(right) and COR2 B(left).

\begin{tabular}{|c|cccccc|}
\hline & $\begin{array}{c}\text { GCS } \\
\text { longitude } \phi\end{array}$ & $\begin{array}{c}\text { GCS } \\
\text { latitude } \theta\end{array}$ & $\begin{array}{c}\text { GCS } \\
\text { asp. ratio } \kappa\end{array}$ & $\begin{array}{c}\text { GCS } \\
\text { half angle } \alpha\end{array}$ & $\begin{array}{c}\text { GCS } \\
\text { tilt } \gamma\end{array}$ & $\begin{array}{c}\text { CME } \\
v_{\text {apex }}\end{array}$ \\
\hline value & $-79^{\circ}$ & $13^{\circ}$ & 0.35 & $28^{\circ}$ & $52^{\circ}$ & $352 \mathrm{~km} / \mathrm{s}$ \\
\hline
\end{tabular}

Table 7.4: GCS fitting parameter and determined CME apex speed of the shown event. GCS longitude and latitude are displayed in the HEEQ coordinate system.
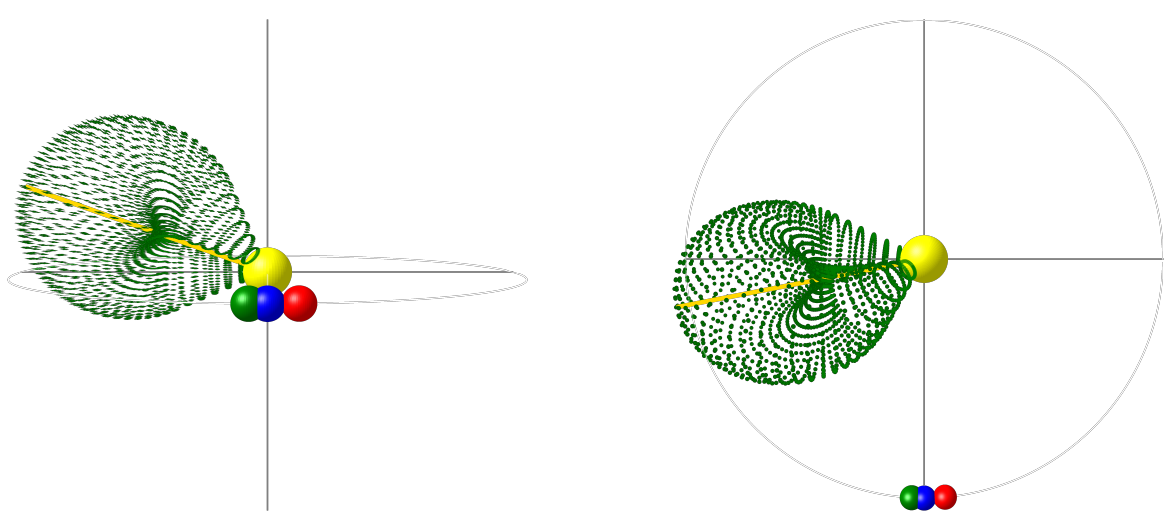

Fig. 7.8: Expansion of 3D GCS grid to an apex height of $1 \mathrm{AU}$ in the HEE coordinate system together with the positions of Earth (blue), STEREO A (red) and STEREO B (green). 


\subsubsection{Sample \#4 - Helcats Event \#63}

Sample event \#4 occurred on 07-04-2011. To this date both STEREO spacecraft have a separation of $175^{\circ}$. The GCS fit of this event is presented in Fig. 7.9 and the fitting parameters in Table 7.5. The orientation of the GCS grid in relation to Earth is presented in Fig. 7.10. The event has a low speed of $444 \mathrm{~km} / \mathrm{s}$ but was modelled with a very extensive structure $\kappa=0.59, \alpha=50^{\circ}$ which is very unusual. Tilting the GCS shape from $0^{\circ}$ to $90^{\circ}$, would probably result in a less extensive morphology. The event was observed with a stereoscopic separation close to $\left(\sigma=180^{\circ}\right)$ degree. In such a configuration, events directed towards $\left(\phi=0^{\circ}\right)$ or away $\left(\phi=180^{\circ}\right)$ from Earth as well as towards one of the STEREO satellites $\left(\phi \approx \pm 90^{\circ}\right)$ have the least amount of stereoscopic resolution. Both observed projective white-light shapes can basically be considered as redundant. Thus, the uncertainty morphology is very high and in this case and fitting with an additional perspective from Earth is highly recommended.
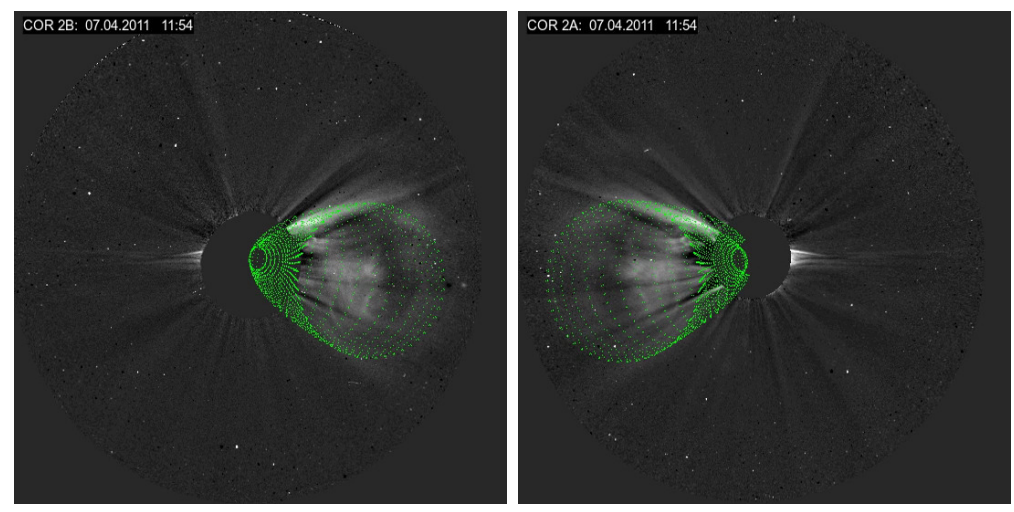

Fig. 7.9: GCS fit of the CME event on 07-04-2011 at 11:54 UT applied to the coronagraph images of STEREO COR2 A(right) and COR2 B(left).

\begin{tabular}{|c|cccccc|}
\hline & $\begin{array}{c}\text { GCS } \\
\text { longitude } \phi\end{array}$ & $\begin{array}{c}\text { GCS } \\
\text { latitude } \theta\end{array}$ & $\begin{array}{c}\text { GCS } \\
\text { asp. ratio } \kappa\end{array}$ & $\begin{array}{c}\text { GCS } \\
\text { half angle } \alpha\end{array}$ & $\begin{array}{c}\text { GCS } \\
\text { tilt } \gamma\end{array}$ & $\begin{array}{c}\text { CME } \\
v_{\text {apex }}\end{array}$ \\
\hline value & $-6^{\circ}$ & $-17^{\circ}$ & 0.59 & $50^{\circ}$ & $0^{\circ}$ & $444 \mathrm{~km} / \mathrm{s}$ \\
\hline
\end{tabular}

Table 7.5: GCS fitting parameter and determined CME apex speed of the shown event. GCS longitude and latitude are displayed in the HEEQ coordinate system.
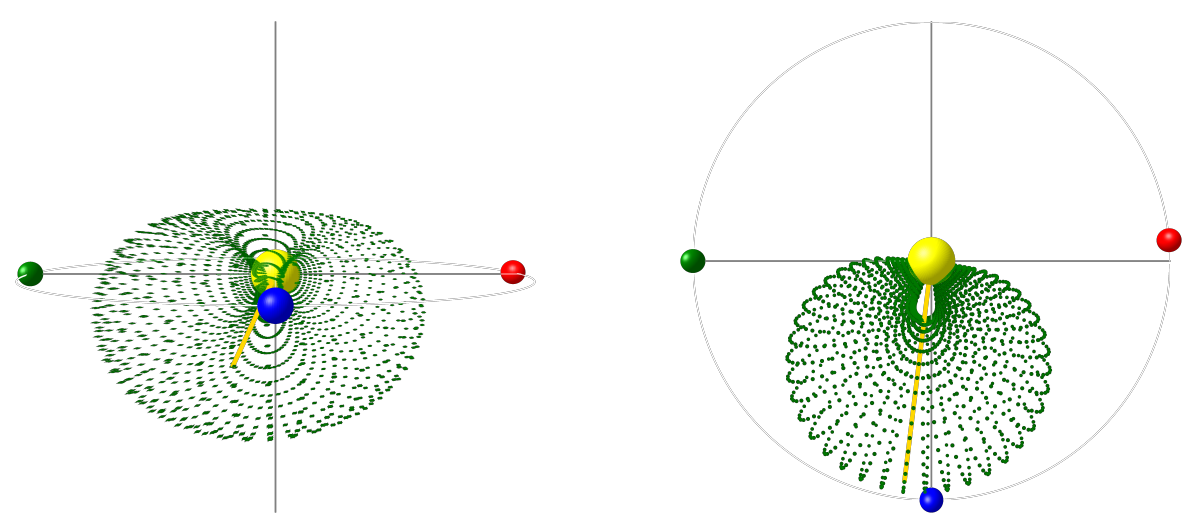

Fig. 7.10: Expansion of 3D GCS grid to an apex height of $1 \mathrm{AU}$ in the HEE coordinate system together with the positions of Earth (blue), STEREO A (red) and STEREO B (green). 


\subsubsection{Sample \#5 - Helcats Event \#49}

Sample event \#5 occurred on 14-12-2010. To this date both STEREO spacecraft have a separation of $173^{\circ}$. The GCS fit of this event is presented in Fig. 7.11 and the fitting parameters in Table 7.6. The orientation of the GCS grid in relation to Earth is presented in Fig. 7.12. The event has the same STEREO satellite configuration as the previous sample. Nevertheless, it is directed at $\phi=50^{\circ}$ and therefore has a sufficient stereoscopic resolution. Above the main GCS shape one can notice a second, slightly fainter brightness enhancement, also reminiscent to a flux rope, which probably results from a second CME. Secondary CMEs can lead to large fitting errors if they are not well distinguished from the original CME.
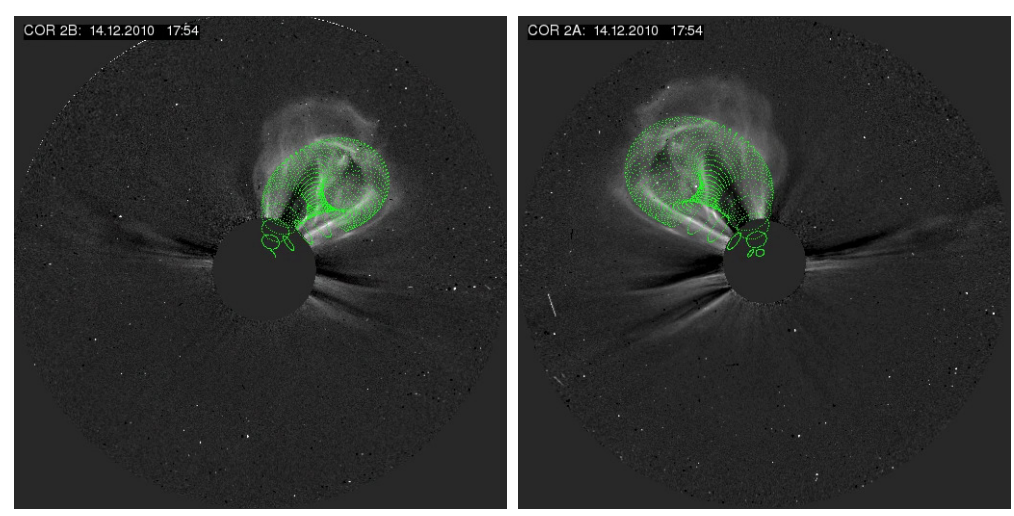

Fig. 7.11: GCS fit of the CME event on 14-12-2010 at 17:54 UT applied to the coronagraph images of STEREO COR2 A(right) and COR2 B(left).

\begin{tabular}{|c|cccccc|}
\hline & $\begin{array}{c}\text { GCS } \\
\text { longitude } \phi\end{array}$ & $\begin{array}{c}\text { GCS } \\
\text { latitude } \theta\end{array}$ & $\begin{array}{c}\text { GCS } \\
\text { asp. ratio } \kappa\end{array}$ & $\begin{array}{c}\text { GCS } \\
\text { half angle } \alpha\end{array}$ & $\begin{array}{c}\text { GCS } \\
\text { tilt } \gamma\end{array}$ & $\begin{array}{c}\text { CME } \\
v_{\text {apex }}\end{array}$ \\
\hline value & $50^{\circ}$ & $30^{\circ}$ & 0.25 & $21^{\circ}$ & $-37^{\circ}$ & $1050 \mathrm{~km} / \mathrm{s}$ \\
\hline
\end{tabular}

Table 7.6: GCS fitting parameter and determined CME apex speed of the shown event. GCS longitude and latitude are displayed in the HEEQ coordinate system.
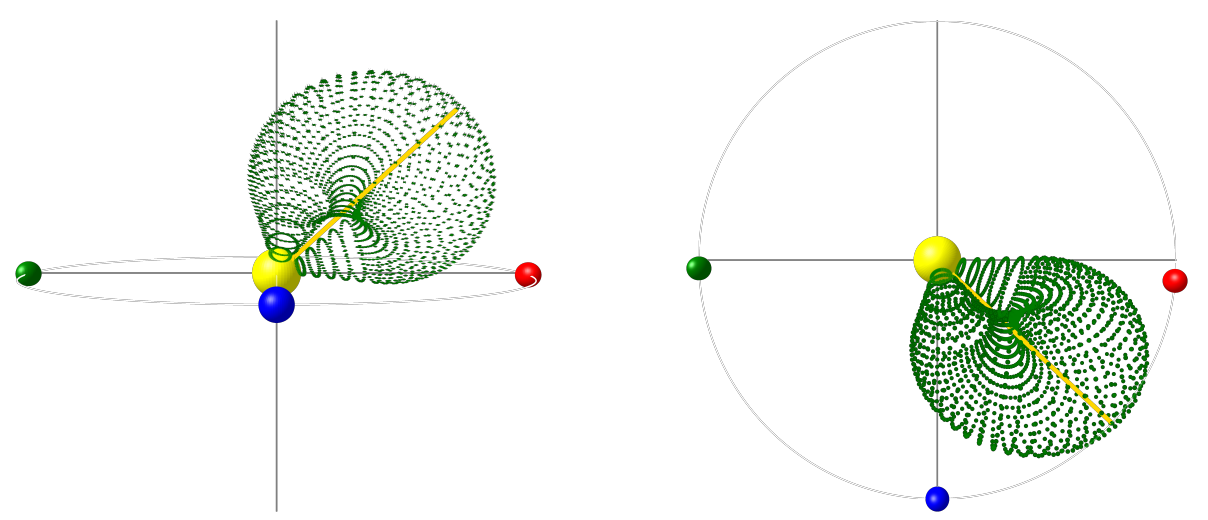

Fig. 7.12: Expansion of 3D GCS grid to an apex height of $1 \mathrm{AU}$ in the HEE coordinate system together with the positions of Earth (blue), STEREO A (red) and STEREO B (green). 


\subsubsection{Sample \#6 - Helcats Event \#73}

Sample event \#6 occurred on 04-06-2011. To this date both STEREO spacecraft have a separation of $172^{\circ}$. The GCS fit of this event is presented in Fig. 7.13 and the fitting parameters in Table 7.7. The orientation of the GCS grid in relation to Earth is presented in Fig. 7.14. The event represents the fastest CME event of the HELCATS Catalogue. It is orientated away from Earth $\left(\phi=130^{\circ}\right)$ and has a very extensive structure. The CME shock is clearly visible as fainter structure, surrounding the main shape. Intense events usually appear during phases of high solar activity, thus a lot of streamers are visible. At the north, a very intense brightness enhancement probably marks the starting emergence of a secondary eruption.
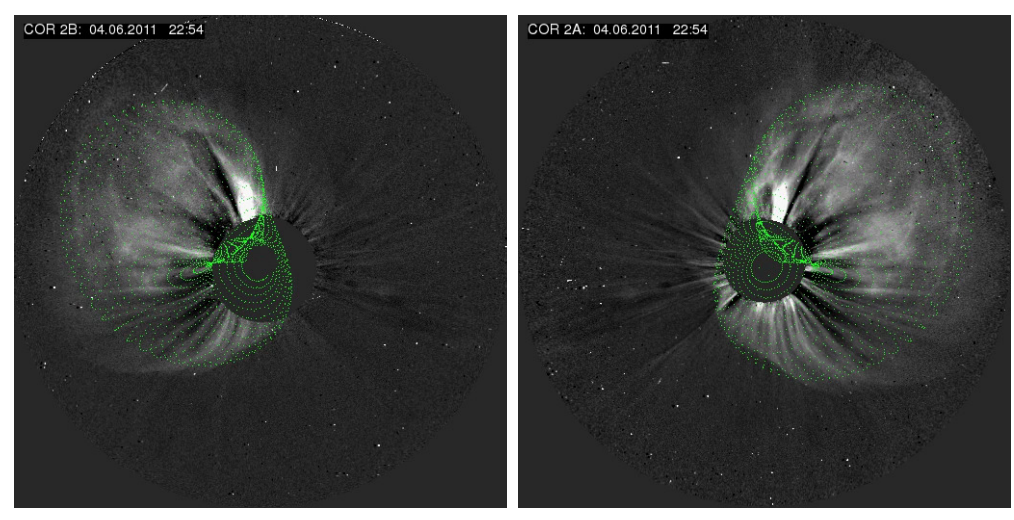

Fig. 7.13: GCS fit of the CME event on 04-06-2011 at 01:07 UT applied to the coronagraph images of STEREO COR2 A(right) and COR2 B(left).

\begin{tabular}{|c|cccccc|}
\hline & $\begin{array}{c}\text { GCS } \\
\text { longitude } \phi\end{array}$ & $\begin{array}{c}\text { GCS } \\
\text { latitude } \theta\end{array}$ & $\begin{array}{c}\text { GCS } \\
\text { asp. ratio } \kappa\end{array}$ & $\begin{array}{c}\text { GCS } \\
\text { half angle } \alpha\end{array}$ & $\begin{array}{c}\text { GCS } \\
\text { tilt } \gamma\end{array}$ & $\begin{array}{c}\text { CME } \\
v_{\text {apex }}\end{array}$ \\
\hline value & $130^{\circ}$ & $19^{\circ}$ & 0.7 & $36.6^{\circ}$ & $55^{\circ}$ & $2616 \mathrm{~km} / \mathrm{s}$ \\
\hline
\end{tabular}

Table 7.7: GCS fitting parameter and determined CME apex speed of the shown event. GCS longitude and latitude are displayed in the HEEQ coordinate system.
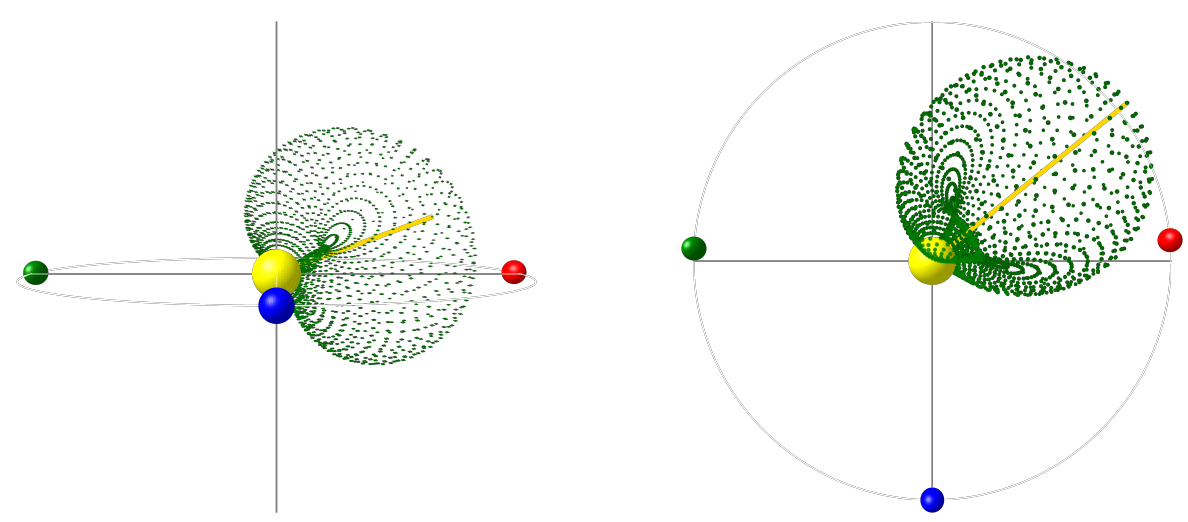

Fig. 7.14: Expansion of 3D GCS grid to an apex height of $1 \mathrm{AU}$ in the HEE coordinate system together with the positions of Earth (blue), STEREO A (red) and STEREO B (green). 


\subsubsection{Sample \#7 - Helcats Event \#78}

Sample event \#7 occurred on 14-06-2011. To this date both STEREO spacecraft have a separation of $172^{\circ}$. The GCS fit of this event is presented in Fig. 7.15 and the fitting parameters in Table 7.8. The orientation of the GCS grid in relation to Earth is presented in Fig. 7.16. The event was fitted with a high $\alpha$ value of $57^{\circ}$ and low $\kappa$ value of 0.28 . It is therefore very reminiscent to a pipe. Despite its unusual shape, the fit appears very trustworthy. One may assume that such elongated structures result from very elongated source regions.
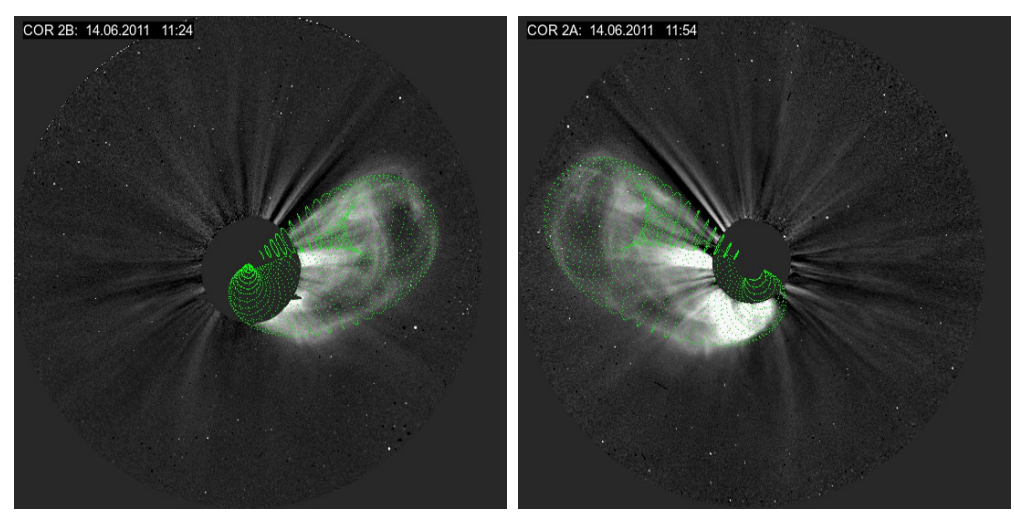

Fig. 7.15: GCS fit of the CME event on 14-06-2011 at 01:07 UT applied to the coronagraph images of STEREO COR2 A(right) and COR2 B(left).

\begin{tabular}{|c|cccccc|}
\hline & $\begin{array}{c}\text { GCS } \\
\text { longitude } \phi\end{array}$ & $\begin{array}{c}\text { GCS } \\
\text { latitude } \theta\end{array}$ & $\begin{array}{c}\text { GCS } \\
\text { asp. ratio } \kappa\end{array}$ & $\begin{array}{c}\text { GCS } \\
\text { half angle } \alpha\end{array}$ & $\begin{array}{c}\text { GCS } \\
\text { tilt } \gamma\end{array}$ & $\begin{array}{c}\text { CME } \\
v_{\text {apex }}\end{array}$ \\
\hline value & $-43^{\circ}$ & $-1^{\circ}$ & 0.28 & $57^{\circ}$ & $36^{\circ}$ & $613 \mathrm{~km} / \mathrm{s}$ \\
\hline
\end{tabular}

Table 7.8: GCS fitting parameter and determined CME apex speed of the shown event. GCS longitude and latitude are displayed in the HEEQ coordinate system.
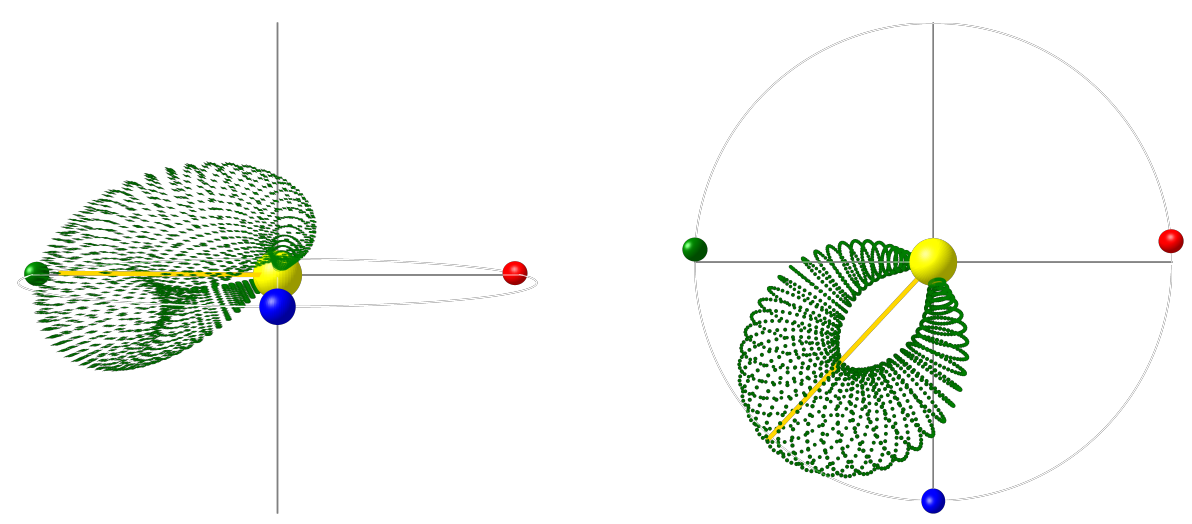

Fig. 7.16: Expansion of 3D GCS grid to an apex height of $1 \mathrm{AU}$ in the HEE coordinate system together with the positions of Earth (blue), STEREO A (red) and STEREO B (green). 


\subsubsection{Sample \#8 - Helcats Event \#81}

Sample event \#8 occurred on 04-08-2011. To this date both STEREO spacecraft have a separation of $167^{\circ}$. The GCS fit of this event is presented in Fig. 7.17 and the fitting parameters in Table 7.9. The orientation of the GCS grid in relation to Earth is presented in Fig. 7.18. This is a very fast event $(1784 \mathrm{~km} / \mathrm{s})$ with a very extensive structure $\left(\kappa=0.79, \alpha=63^{\circ}\right)$. In this case, it seems that the shape was slightly overestimated in the north by the brightness enhancement of a shock front. It appears that fitting CMEs at very low heights is beneficial to identify CME fronts as the shock has not yet surpassed the front.
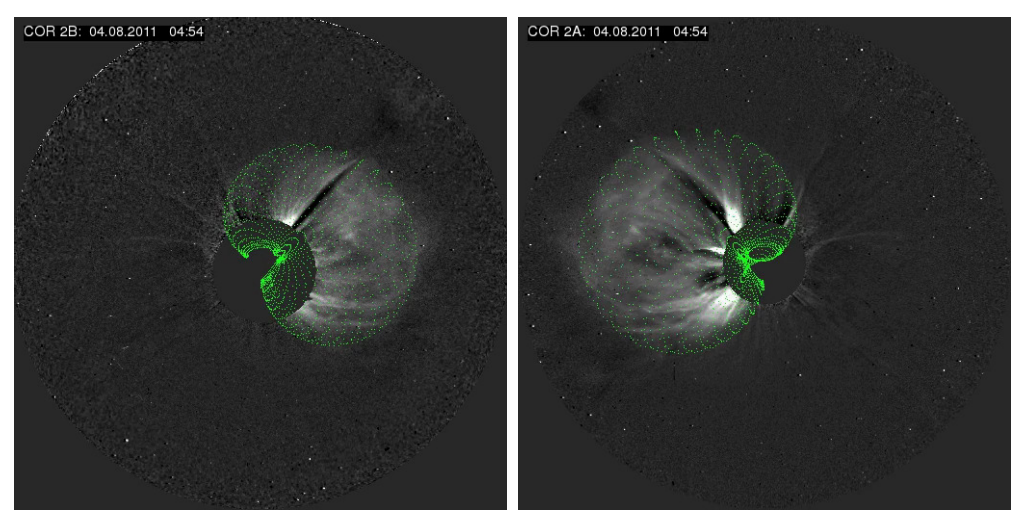

Fig. 7.17: GCS fit of the CME event on 04-08-2011 at 01:07 UT applied to the coronagraph images of STEREO COR2 A(right) and COR2 B(left).

\begin{tabular}{|c|cccccc|}
\hline & $\begin{array}{c}\text { GCS } \\
\text { longitude } \phi\end{array}$ & $\begin{array}{c}\text { GCS } \\
\text { latitude } \theta\end{array}$ & $\begin{array}{c}\text { GCS } \\
\text { asp. ratio } \kappa\end{array}$ & $\begin{array}{c}\text { GCS } \\
\text { half angle } \alpha\end{array}$ & $\begin{array}{c}\text { GCS } \\
\text { tilt } \gamma\end{array}$ & $\begin{array}{c}\text { CME } \\
v_{\text {apex }}\end{array}$ \\
\hline value & $35^{\circ}$ & $22^{\circ}$ & 0.79 & $63^{\circ}$ & $60^{\circ}$ & $1784 \mathrm{~km} / \mathrm{s}$ \\
\hline
\end{tabular}

Table 7.9: GCS fitting parameter and determined CME apex speed of the shown event. GCS longitude and latitude are displayed in the HEEQ coordinate system.
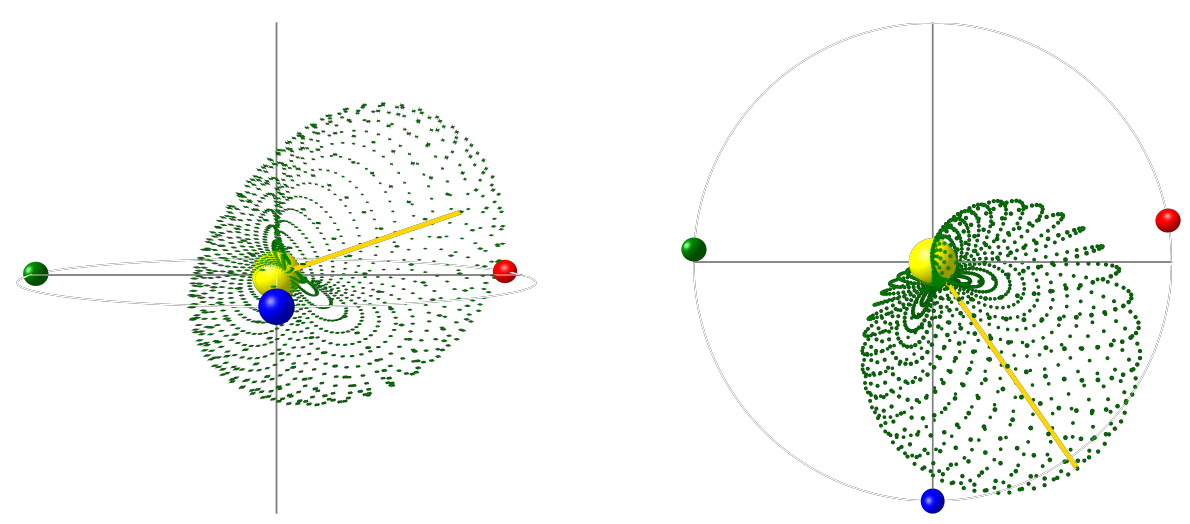

Fig. 7.18: Expansion of 3D GCS grid to an apex height of $1 \mathrm{AU}$ in the HEE coordinate system together with the positions of Earth (blue), STEREO A (red) and STEREO B (green). 


\subsubsection{Sample \#9 - Helcats Event \#112}

Sample event \#9 occurred on 17-07-2012. To this date both STEREO spacecraft have a separation of $145^{\circ}$. The GCS fit of this event is presented in Fig. 7.19 and the fitting parameters in Table 7.10. The orientation of the GCS grid in relation to Earth is presented in Fig. 7.20. This event represents one of the most difficult GCS fits. The displayed CME posses a very ambiguous flux rope structure which can be easily seen in COR2 A by the existence of a strongly kinked CME front. This makes the application of the GCS model very questionable, which is only designed for an ideal flux rope morphology. Moreover, the second CME clearly appearing at solar west in the COR2 A, seems to further contribute to the overestimation of the shape in COR2 B.
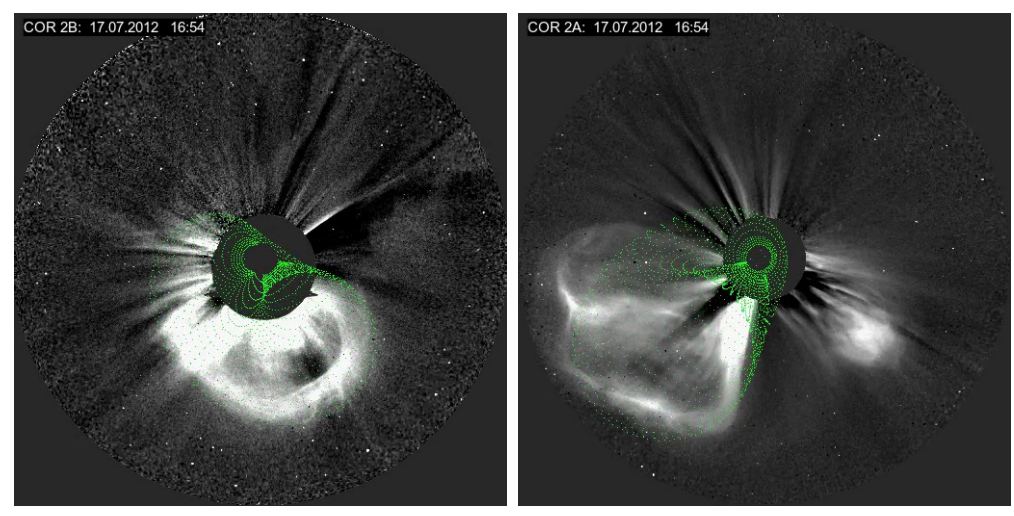

Fig. 7.19: GCS fit of the CME event on 17-07-2012 at 01:07 UT applied to the coronagraph images of STEREO COR2 A(right) and COR2 B(left).

\begin{tabular}{|c|cccccc|}
\hline & $\begin{array}{c}\text { GCS } \\
\text { longitude } \phi\end{array}$ & $\begin{array}{c}\text { GCS } \\
\text { latitude } \theta\end{array}$ & $\begin{array}{c}\text { GCS } \\
\text { asp. ratio } \kappa\end{array}$ & $\begin{array}{c}\text { GCS } \\
\text { half angle } \alpha\end{array}$ & $\begin{array}{c}\text { GCS } \\
\text { tilt } \gamma\end{array}$ & $\begin{array}{c}\text { CME } \\
v_{\text {apex }}\end{array}$ \\
\hline value & $63^{\circ}$ & $-28^{\circ}$ & 0.63 & $31^{\circ}$ & $35^{\circ}$ & $1011 \mathrm{~km} / \mathrm{s}$ \\
\hline
\end{tabular}

Table 7.10: GCS fitting parameter and determined CME apex speed of the shown event. GCS longitude and latitude are displayed in the HEEQ coordinate system.
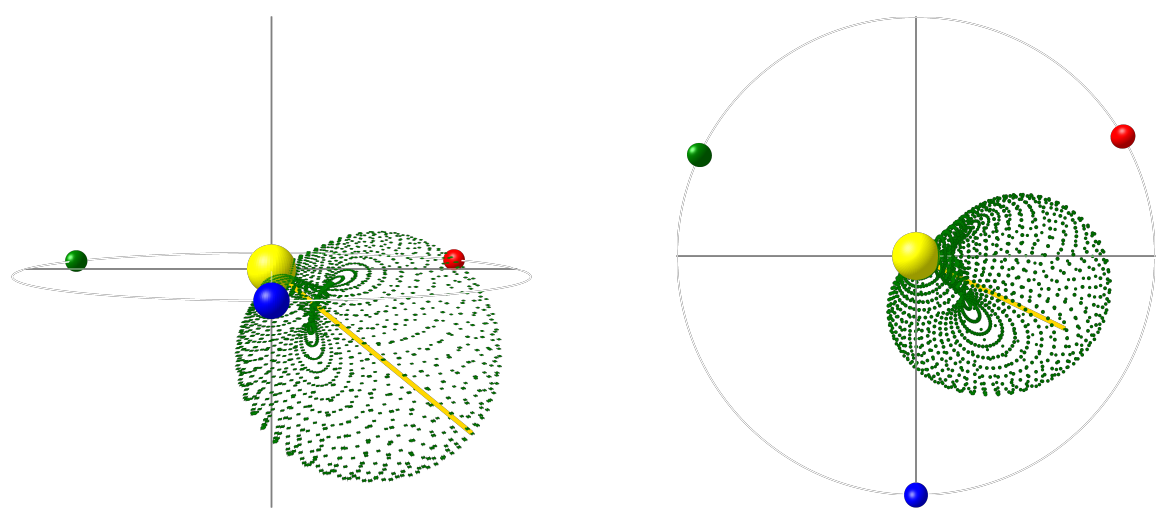

Fig. 7.20: Expansion of 3D GCS grid to an apex height of $1 \mathrm{AU}$ in the HEE coordinate system together with the positions of Earth (blue), STEREO A (red) and STEREO B (green). 


\subsubsection{Sample \#10 - Helcats Event \#117 - Date 17-05-2013}

Sample event \#10 occurred on 17-05-2013. To this date both STEREO spacecraft have a separation of $92^{\circ}$. The GCS fit of this event is presented in Fig. 7.21 and the fitting parameters in Table 7.11. The orientation of the GCS grid in relation to Earth is presented in Fig. 7.22. The event is very fast $(1585 \mathrm{~km} / \mathrm{s})$ and occured during a phase of high solar activity. Compared to the high CME velocity, the fitted flux rope structure $\left(\kappa=0.33, \alpha=13.9^{\circ}\right)$ appears very narrow which might be a significant underestimation. The high solar activity, makes a correct interpretation of this structure very difficult without taking further perspectives into account.
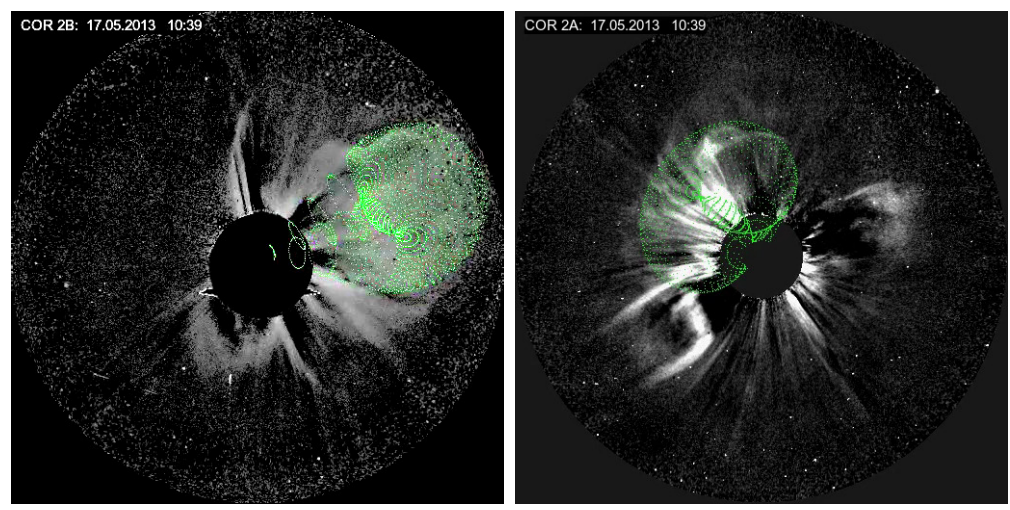

Fig. 7.21: GCS fit of the CME event on 17-05-2013 at 01:07 UT applied to the coronagraph images of STEREO COR2 A(right) and COR2 B(left).

\begin{tabular}{|c|cccccc|}
\hline & $\begin{array}{c}\text { GCS } \\
\text { longitude } \phi\end{array}$ & $\begin{array}{c}\text { GCS } \\
\text { latitude } \theta\end{array}$ & $\begin{array}{c}\text { GCS } \\
\text { asp. ratio } \kappa\end{array}$ & $\begin{array}{c}\text { GCS } \\
\text { half angle } \alpha\end{array}$ & $\begin{array}{c}\text { GCS } \\
\text { tilt } \gamma\end{array}$ & $\begin{array}{c}\text { CME } \\
v_{\text {apex }}\end{array}$ \\
\hline value & $-29^{\circ}$ & $12^{\circ}$ & 0.33 & $13.9^{\circ}$ & $-52^{\circ}$ & $1588 \mathrm{~km} / \mathrm{s}$ \\
\hline
\end{tabular}

Table 7.11: GCS fitting parameter and determined CME apex speed of the shown event. GCS longitude and latitude are displayed in the HEEQ coordinate system.
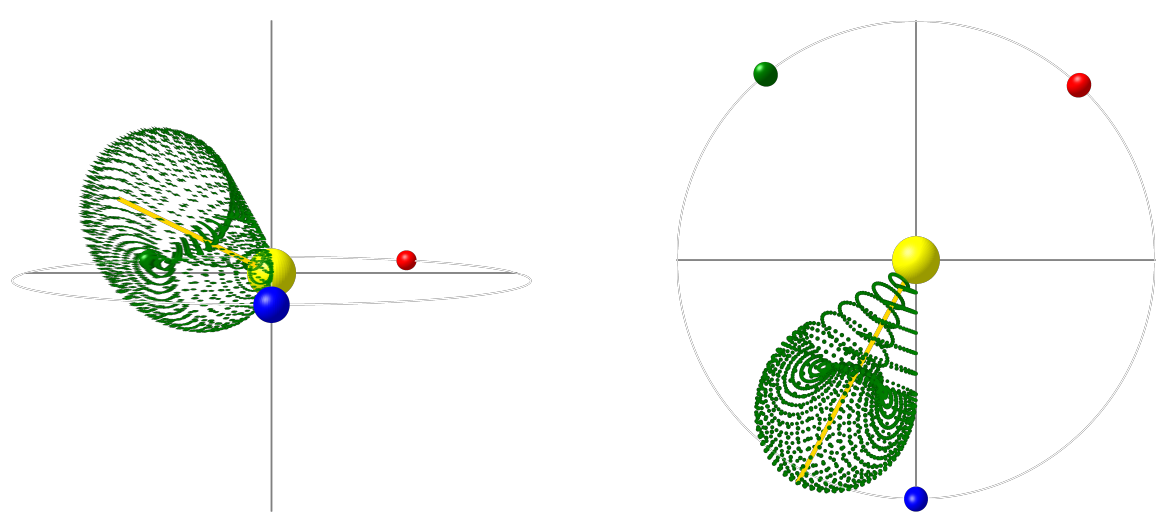

Fig. 7.22: Expansion of 3D GCS grid to an apex height of $1 \mathrm{AU}$ in the HEE coordinate system together with the positions of Earth (blue), STEREO A (red) and STEREO B (green). 


\section{Conclusion}

The statistical GCS results for the whole set of 122 CMEs are presented in a more comprehensive manner together with the mass determination results in Chapter 8. The shown sample events have illustrated some of the common difficulties in the geometrical modelling of CMEs by stereoscopic observations with STEREO. These difficulties are ranked by their expected relevance.

\section{Instrument Separation}

First of all, it has to be noted that stereoscopic observations are obviously only useful for a sufficient large stereoscopic angle. Often an angle of more than $15^{\circ}$ is considered as sufficient for other stereoscopic applications. On the other hand, separation angles close to $180^{\circ}$ can also be problematic, if a CME is directed towards one observer or in a close to $90^{\circ}$ angle. In such cases both observed projective white-light shapes can basically be considered as redundant so that the already discussed projection effect for a single perspective observation apply.

\section{Missing Flux Rope Structure}

In Chapter 4 it was discussed that to this day it is not clear if all CMEs posses a flux rope structure. In other cases the flux rope structure might be disturbed or even a combination of more than one flux rope. In these cases the fitting of a simple flux rope with the GCS model is not appropriate and the deprojected geometry can lead to a huge bias.

\section{Coronal Shock Waves}

It was demonstrated that the brightness enhancements caused by a CME shock wave can be easily mistaken for the CME front. The likeliness of such shock waves rises with the CME speed and width. Distinguishing the CME front and the CME shock front is particularly difficult if the event is observed as "Halo" in both coronagraphs.

\section{Secondary CMEs}

It was shown that secondary CMEs can lead to a misinterpretation of the morphology of the originally attempted modelling target. This can lead to large geometrical overestimation, thus both CMEs need to be well distinguished from each other. The fitting difficulties of such scenarios vary with the observing perspectives as well as the morphology, the speed and the propagation direction of both CMEs.

\section{Coronal Streamer}

It could not be shown that coronal streamers have any significant effects on the modelling results of the CME morphology or CME speed. Though, this possibility cannot be generally excluded. However, coronal streamers are a frequently occuring phenomenon and can complicate the visual interpretation of CME structures, especially in conjunction with some of the other four points mentioned above. 


\title{
8 Combining GCS Modelling and CME Mass Determination
}

\begin{abstract}
This chapter starts with an overview of former CME mass estimation studies. This is followed by the presentation of a newly established combined mass determination and geometrical modelling method. The method is applied to 122 CME events and the modelling results are compiled to an online database. The modelling results are statistically analysed to derive important parameter correlations, which are relevant in the context of CME forecasts. It is further shown that large CME mass overestimations can arise from the presence of coronal streamer. Two such events from the catalogue are therefore presented in more detail. Finally, the derived mass results from the catalogue are used to estimate the total solar mass loss caused by CMEs and compare it to other sources of solar mass loss.
\end{abstract}

\subsection{CME Mass Determination}

In the past, the measurement of CME masses based on the CME white-light appearance was subject of many studies. The underlying mechanism is known as Thomson scattering as confirmed by Bowles (1958). Munro et al. (1979) and Poland et al. (1981) were among the first to measure CME masses through scattering theory using space coronagraphs onboard of SKYLAB (Tousey, 1977) and SOLWIND (Sheeley et al., 1980). Vourlidas et al. (2010) found that the majority of CMEs have masses in the range of $10^{13}-10^{16} \mathrm{~g}$ through analysing over 7000 CMEs with the LASCO/SOHO (Brueckner et al., 1995b) coronagraph during solar cycle 23. Other mass estimation CME methods like radio telescoped based measurements of the thermal bremsstrahlung (Gopalswamy \& Kundu, 1992) of CME mass determination by EUV Dimming Aschwanden et al. (2009) show comparable results.

Previous white-light based mass measurements were effected by high uncertainties. Those mainly arise due to observations that were made based on only a single viewing point. The necessary accuracy in the determination of the CME geometry and position is necessary, as both have an important impact on the Thomson scattering equation and therefore also on the determination of the total CME mass. With the advent of the STEREO satellites, the geometry and position of CMEs can be determined with a much higher precision allowing to eliminate many sources of uncertainty. After analysing over 7000 CMEs with the LASCO/SOHO coronagraph during solar cycle 23, Vourlidas et al. (2010) reported that the majority of CMEs have masses in the range of $10^{13}-10^{16} \mathrm{~g}$ and kinetic energy ranges from $10^{29}$ to $10^{32} \mathrm{ergs}$. The so far largest kinetic energy observed was $1.2 \times 10^{33} \mathrm{erg}$ for the Halloween Storm on October 282003 (Gopalswamy et al., 2005) causing strong geomagnetical disturbances (Cid et al., 2015). 


\subsection{Projection Effects and CME Parameter Study}

Based on the theory of the previous chapters, a combined method of GCS modelling and CME white-light mass determination was established. The demonstrated method enables the measurement of relevant kinematic CME parameters in a consistent and comparable manner. The method was applied to a set of 122 events with intense brightness, which are stereoscopically observed with the COR2 coronagraphs onboard of the two NASA STEREO spacecrafts. The result were compiled in a kinematic online catalog within the EU FP7 project HELCATS. The statistical CME parameter results are analysed to derive their mutual correlation, their correlation to the solar activity cycle as well as the influence of projection effects. Further inherent peculiarities and implications for the mass determination of events emerging from close to the disk centre towards or away from the observer are discussed.

This section presents and discusses a combined method GCS modelling and CME whitelight mass determination applied method and its statistical results. It was submitted to Astronomy and Astrophysics (A\&A) on July 9, 2018. The co-authors contributed to this section in the following manner:

- Niclas Mrotzek, Angelos Vourlidas and Volker Bothmer supervised the formal structure of the paper, contributed critical comments on the theory and helped with the interpretation of the analysis.

- Neel Savani provided the software routine of the presented method.

- All co-authors have also additionally contributed with language editing.

The modelling results have been stored in the Kinematic Database Catalogue (KinCat). KinCat was established as one contribution of the University of Göttingen to the European Community's Seventh Framework Programme (FP7) "Heliospheric Cataloguing, Analysis and Techniques Service (HELCATS)".

The following persons have contributed to the creation of KINCAT:

- Dr. Volker Bothmer (project leader)

- Adam Pluta (CME modelling, online database)

- Niclas Mrotzek (CME modelling)

- Julius Achenbach (online database)

- Dr. Eckhardt Bosman (event selection)

As part of the more comprehensive HELCATS catalogue (Harrison et al., 2016), KinCat is cross-linked via a unique identifier (ID) to other sub-catalogues. This includes catalogues of heliospheric observations, in-situ and automated coronagraphic detections as well as source regions and low coronal events. The full HELCATS catalogue ${ }^{1}$ as well as the KinCat catalogue ${ }^{2}$ are publicly accessible.

\footnotetext{
${ }^{1}$ https://www.helcats-fp7.eu/catalogues/wp3_kincat.html

${ }^{2}$ http://www.affects-fp7.eu/helcats-database/database.php
} 


\title{
Combined Geometrical Modelling and White-Light Mass Determination of Coronal Mass Ejections
}

\author{
Adam Pluta ${ }^{1}$, Niclas Mrotzek $^{1}$, Angelos Vourlidas ${ }^{2,3}$, Volker Bothmer $^{1}$, Neel Savani ${ }^{4,5}$ \\ 1 Institute for Astrophysics, University of Göttingen, Göttingen, Germany \\ 2 Johns Hopkins University Applied Physics Lab, Laurel, MD, USA \\ 3 IAASARS, Observatory of Athens, Athens, Greece \\ 4 NASA Goddard Space Flight Center, Greenbelt, MD, USA \\ 5 Goddard Planetary Heliophysics Institute (GPHI),University of Maryland, BL, MD, USA \\ Submitted, July 9, 2018
}

\section{ABSTRACT}

Context. We use forward modelling on multi-viewpoint coronagraph observations to estimate the 3-dimensional morphology, initial speed and deprojected masses of Coronal Mass Ejections (CMEs). The CME structure is described via the Graduated Cylindrical Shell (GCS) model, which enables the measurement of CME parameters in a consistent and comparable manner.

Aims. This is the first large-scale use of the GCS model to estimate CME masses, so we discuss inherent peculiarities and implications for the mass determination with a special focus on CME events emerging from close to the observer's central meridian. Further, we analyse the CME characteristics best suited to estimate the CME mass in a timely manner to make it available to CME arrival predictions.

Methods. We apply the method to a set of 122 bright events observed simultaneously from two vantage points with the COR2 coronagraphs onboard of the twin NASA STEREO spacecraft. The events occurred between January 2007 and December 2013 and are compiled in an online catalogue within the EU FP7 project HELCATS. We statistically analyse the derived CME parameters, their mutual connection and their relation to the solar cycle.

Results. We show that the derived morphology of intense disk events is still systematically overestimated by up to a factor of 2 with stereoscopic modelling, which is the same order of magnitude as for observations from only one vantage point. The overestimation is very likely a combination of projection effects as well as the increased complexity of separating CME shocks and streamers from CME fronts for such events. We further show that CME mass determination of disk events can lead to overestimation of the mass by about a factor of 10 or more, in case of overlapping bright structures. Conclusions. We conclude that for stereoscopic measurements of disk events, the measurement of the initial CME speed is the most reliable one. We further suggest that our presented CME speed-mass correlation is most suited to estimate the CME mass early from coronagraph observations.

Key words. Sun: coronal mass ejections (CMEs) - Sun: corona - Sun: solar-terrestrial relations - Sun: heliosphere

\section{Introduction}

Coronal Mass Ejections (CMEs) are explosive large-scale outbursts of the Sun's coronal plasma and magnetic field. They can induce strong geomagnetic storms at Earth (Tsurutani et al. 1988; Gosling et al. 1991), which pose serious threats to space systems, communications and navigation (Cherry 2002; Thomson et al. 2011; Pulkkinen et al. 2005; Burlaga et al. 1987; Thomson et al. 2011). Therefore, precise measurements of CME kinematics are important to predict their arrival at Earth. Besides knowledge about the prevailing solar wind conditions, the performance of CME propagation models relies on accurate measurements of the CME initial geometry and CME apex speed as well as the CME mass. For example, the Drag-Based Model (DBM) (Vršnak et al. 2013), which is based on the hydrodynamic analogue of the aerodynamic drag acting between the solar wind and the CME, depends on these properties. To maximise the benefit for space weather predictions, CME kinematics and geometry have to be determined as early as possibly during the CME emergence. Coronagraph observations can provide these values at CME distances within a few solar radii away from the Sun. Projection effects are discussed often in the context of CME kinematics (Burkepile et al. 2004; Vršnak et al. 2007a; Temmer et al. 2009; Liu et al. 2010a; Shen et al. 2013a; Davies et al. 2013), masses (Vourlidas et al. 2010) and morphologies (Liu et al. 2010b; Savani et al. 2011a,b; Vourlidas et al. 2013; Kwon et al. 2015). Projection effects can result in an overestimation of the CME size and an underestimation of the CME velocity.

We do not consider projection effects resulting from the approximation of the Thomson sphere with the plane-of-sky (POS) since those only become important beyond $120 \mathrm{R}$. (e.g., Fig. 2 in Vourlidas \& Howard 2006). Nevertheless, projection effects associated with Thomson scattering also affect the CME mass determination which is discussed in more detail in Section 3.3. These effects are inherent due to the 3-dimensional CME morphology and become extreme for the so-called "halo events". The term "halo" refers to CMEs that exhibit a circular or elliptical white-light morphology encircling the Sun (Howard et al. 1982). The 
halo appearance generally implies that the event is directed along the Sun-observer line and most likely arise from the CME-driven shock (Kwon et al. 2015). We adopt the term "disk events" to characterise events originating from close to an observer's solar disk centre, propagating along the Sun-observer line towards or away to the observer. The kinematic and morphological characteristics of disk events, such as the height, width and propagation speed suffer from severe projection effects. These projection effects have been assessed via multi-viewpoint geometrical modelling (Thernisien et al. 2009; Liewer et al. 2011; Temmer et al. 2009; Lee et al. 2014).

With the launch of NASA's Solar TErrestrial Relations Observatory (STEREO) mission in the October 2006 (Kaiser et al. 2008) long-term multi-viewpoint observations of CMEs have been made possible. The STEREO mission consists of two nearly identical spacecraft. The STEREOA orbit is closer to the Sun than the STEREO-B orbit, which results in STEREO-A pulling further ahead of Earth and STEREO-B falling successively behind Earth at a rate of about $22.5^{\circ}$ per year. Each spacecraft carries the SunEarth Connection Coronal and Heliospheric Investigation (SECCHI) (Howard et al. 2008) payload that consists of one extreme ultraviolet (EUV) full disk imager, two coronagraphs - COR1 and COR2, and two heliospheric imagers - HI1 and HI2. In this work, the white-light images of the SECCHI/COR2 coronagraphs are used for the geometrical modelling as well as for the CME mass determination. Vourlidas et al. (2010)(V10, hereafter) discuss extensively the methodology, caveats, and historical background of mass measurements. They specifically exclude halo events from the analysis, to avoid significant mass overestimations resulting from projection effects for single viewpoint measurements. The objective of the present study is to analyse the reliability of CME mass-determinations in the context of projection effects using multi-viewpoint measurements. To achieve this goal, we combine multi-viewpoint geometrical modelling with mass-determination and compare the mass results for disk and limb events to identify possible sources of mass overestimation. Our approach is also used to provide important CME parameter correlations between the CME mass and the initial CME speed close to the Sun. Such correlations are important to estimate CME masses in real-time, in order to make reasonable assumptions in CME prediction models, such as the DBM or WSA-ENLIL plus Cone Model (Odstrčil \& Pizzo 1999; Odstrcil et al. 2004; Mays et al. 2015). For instance, in the DBM the CME deceleration increases inversely with the CME mass.

The paper is organised as follows: In Section 2, we present our measurement methodology in detail. This includes event selection criteria, image processing, application of geometrical forward modelling and the CME mass determination technique. We use an example event to demonstrate the methodology. In Section 3, we provide a statistical analysis of the data set. The results are discussed in Section 4 and compared to previous single-viewpoint studies. We conclude in Section 5 .

\section{Methodology}

\subsection{Event Selection}

We model 122 CME events, which occurred between January 2007 and December 2013, using data from the SECCHI/COR2 coronagraphs. The STEREO satellite geometry for the observed events is presented in Fig. 1. All events are assembled in the Coronal Mass Ejection Kinematic Database Catalogue (KinCat). As part of the more comprehensive HELCATS catalogue (Harrison et al. 2016), KinCat is cross-linked via a unique identifier (ID) to other sub-catalogues. This includes catalogues of heliospheric observations, in-situ and automated coronagraphic detections as well as source regions and low coronal events. The full HELCATS catalogue is accessible at https:// www.helcats-fp7.eu/catalogues/wp3_kincat.html and the KinCat catalogue at http://www.affects-fp7.eu/ helcats-database/database.php.

In addition to kinematic, geometric and mass parameters, KinCat also provides animated fitting sequences, height-time profiles and the STEREO observing geometry for each event. It is based on the first multi-viewpoint CME catalogue, compiled at the University of Göttingen. The catalogue contains 1060 events from 2007 to 2011 and a subset of 263 events was selected based on subjective clear visibility and assembled in the "Best-of" list. From this list, 243 events were modelled (Bosman \& Bothmer 2012; Bosman et al. 2012) with GCS modelling technique developed by Thernisien, Howard and Vourlidas (2006) at the point in time of their clearest visual appearance. Starting from the 243 events, we compare them with the Rutherford Appleton Laboratory Heliospheric Imager (RAL-HI) event-list which contains over 1000 entries within the relevant time period. This step ensures that our events can also be used for more comprehensive studies which follows the purpose of the HELCATS project. As a result of the comparison, 109 events out of 243 events were matched with the HI list. The list is extended by another 13 events between 2012 and 2013. These events are associated with Earthdirected CMEs and are chosen from the HELCATS linked catalogue called LINKCAT (Kilpua et al. 2017) which connects heliospheric and in-situ CME observations. For each single event, we have not noticed any brightness enhancements within the FOV of COR2 which might suggest possible interactions with other CMEs.

\subsection{Data Processing}

We start with the COR2 Level-0.5 FITS images, which are obtained from the NASA Goddard Space Flight Center ${ }^{2}$. Level-0.5 FITS images are already aligned to the ecliptic north. They are further processed to Level-1.0 FITS images via the IDL SolarSoft routine secchi_prep.pro. The resulting images are normalised by the exposure duration, the correction of on-board image processing, the subtraction of the CCD bias, the conversion to the physical units of mean solar brightness (MSB), vignetting and flat-field corrections and other optical distortion corrections (Howard et al. 2008). Then a suitable pre-event image, generally the last image prior to the appearance of the CME in the particular coronagraph's field of view, is subtracted from

\footnotetext{
0 https://stereo-ssc.nascom.nasa.gov/where/

2 ftp://stereoftp.nascom.nasa.gov/pub/
} 


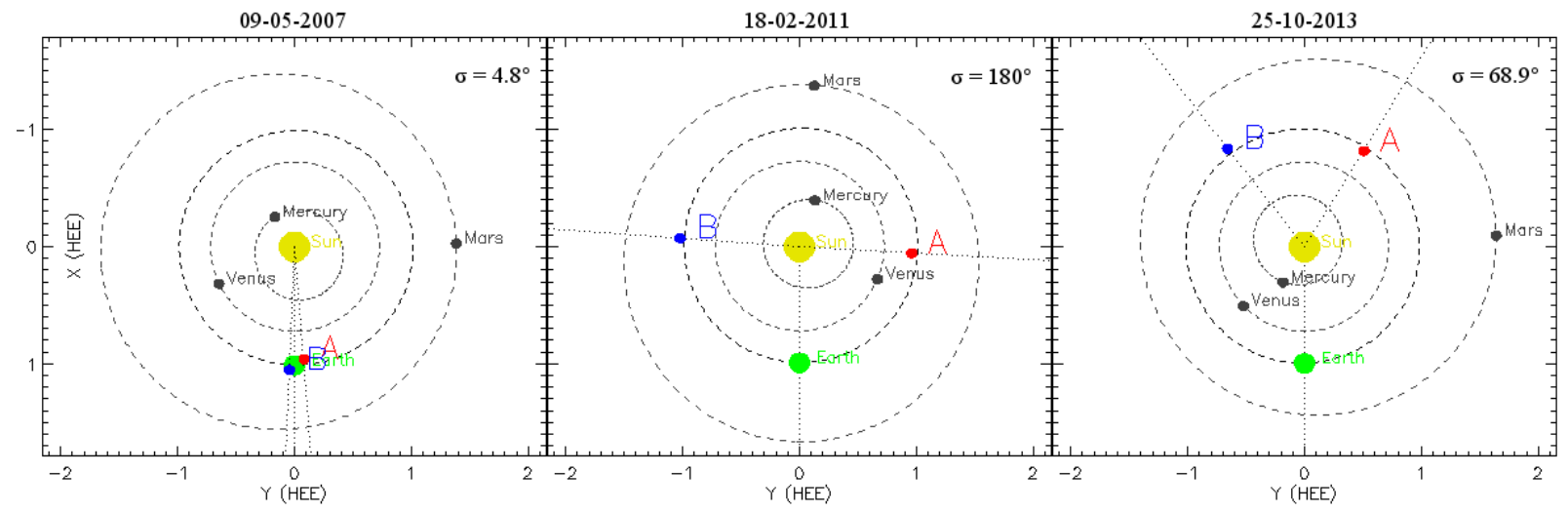

Figure 1. Evolution of STEREO spacecraft separation from the first catalogue event, over the STEREO opposition phase to the last event. The shown minimal STEREO separation (MSS) angle $\sigma$ is defined as the minimal angular distance between both STEREO satellites.(STEREO Science Center) ${ }^{1}$

the CME images to remove the background corona. In order to avoid visual signatures of coronal streamers and other CMEs, it can sometimes be reasonable to subtract an earlier image instead (see V10 for details). We then apply Thomson scattering theory to Level-1.0 FITS images to create so called "mass images". This translation of the images from the unit of MSB to grams per pixel is realised with the make_mass_fits.pro routine of the Solarsoft library. The exact procedure is described in Savani et al. (2013). Details and issues arising in the CME mass calculations are described in V10. For completeness we provide a brief summary below.

\subsubsection{Mass Determination}

Obviously, all CME white-light observations are projections of the 3-dimensional CME structures onto the POS of the observing instrument. Two basic geometrical approximations are traditionally applied that result in a lower limit estimate of the CME mass:

1. All CME material is confined onto a 2-dimensional plane along the direction with the longitudinal angle $\phi$ away from the POS.

2. The plane is assumed to be positioned on the POS $\left(\phi=0^{\circ}\right)$.

V10 analysed the consequences from both assumptions and showed that the POS assumption yields a lower limit mass estimation. The underestimation increases with the $\mathrm{CME}$ width and reaches a factor of two for a CME with $60^{\circ}$ angular width. In this work, we call this approach "POS mass determination". V10 also numerically analysed the results of the applied scattering theory for the case where $\phi$ is equal to the actual longitudinal CME propagation angle away from the POS. They found that this improves the accuracy of the mass measurements relative to POS mass determination up to a propagation angle of $\phi=60^{\circ}$. From there on, the CME mass becomes exponentially overestimated with increasing $\phi$. In the case of a CME propagating at $\phi=80^{\circ}$ (disk event) and possessing a width of $60^{\circ}$, the CME mass could be overestimated by about a factor of five. For that reason, this approach can be used to determine an upper limit of the CME mass, in particular for disk events. In this study, we call this approach "directional mass determination". Previous stereoscopic mass studies by Colaninno \& Vourlidas (2009); Bein et al. (2013) and de Koning (2017) are not combined with geometrical modelling. They use an inverse approach by comparing the mass results in dependance of the unknown propagation angle $\phi$ for each coronagraph and deliver the correspondent mass result to that angle. In this study $\phi$ is determine from geometrical modelling, which is a very established method for this purpose.

\subsection{Graduated Cylindrical Shell Model}

We apply the Graduated Cylindrical Shell (GCS) model (Thernisien et al. 2009) as our geometrical modelling method to COR2 images to derive their 3D size and direction as a function of height. The model represents a simplified geometric proxy to a magnetic flux rope topology. This CME topology has been suggested by many authors (e.g. Chen et al. 1997; Vourlidas et al. 2000; Cremades \& Bothmer 2004). Recently, Vourlidas et al. (2013) have shown that it applies to at least $40 \%$ of all CMEs. It was further suggested that the absence of such a structure can likely be traced back to observational effects (Gopalswamy 2013). The shape of the model is reminiscent of a hollow croissant and the basic geometry consists of two cone shaped legs, separated by twice the half angle $\alpha$ and the leg height $h$. The circular annulus in the middle of the tube section is given by $a(r)=\kappa r$, where $r$ is the distance from the solar centre to a point of the outer edge of the shape and $\kappa$ is the aspect ratio. The origin of the model is rooted at the solar centre. Its main axis enters the solar surface at a point given by longitude $\phi$, latitude $\theta$. The rotation along the main axis is described with the tilt angle $\gamma$. In the full set of GCS parameters it is common to substitute the leg height $h$ with the apex height $h_{\text {apex }}$. The GCS modelling is performed with the rtsccguicloud.pro routine in the SECCHI analysis tree of the IDL SolarSoft distribution. The routine enables us to adjust the wire grid simultaneously onto the white-light CME structures in both COR2 coronagraphs. The projection of the shape is plotted over each coronagraph image with respect to the STEREO satel- 
lite configuration, as demonstrated with the sample event in Section 2.5.
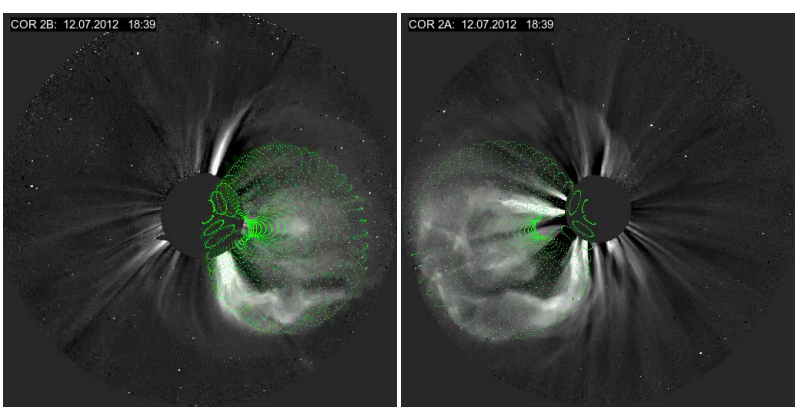

Figure 2. GCS model fitted onto the coronagraph images of SECCHI/COR2 B (left) and COR2 A (right) for the CME event on July 12,2012 at $18.39 \mathrm{UT}$.

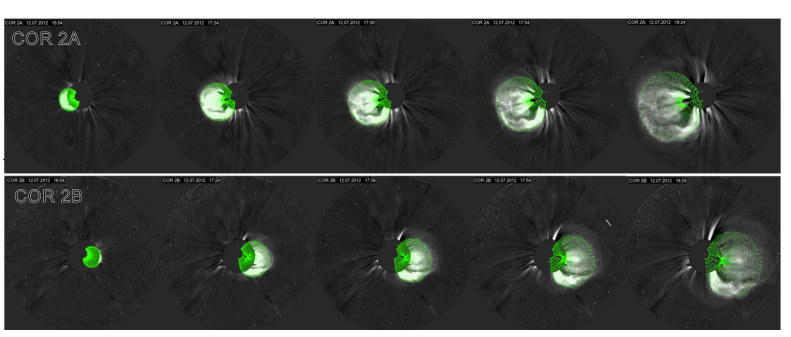

Figure 3. GCS fitting on a sequence of COR2 A (top) and COR2 B (bottom) images of the STEREO/SECCHI coronagraphs for the July 12, 2012 event from 16:54 to 18:24 UT.

\subsection{Geometrical Modelling}

The GCS model is applied to the full COR2 observation sequence which later will be also used to derive CME speeds. Further, the events from the original list are fitted at the height of their individual best white-light visibility. For our analysis, we define a reference height at which the CME properties are compared to each other, in order to reduce evolutionary effects of the CME parameters. Vourlidas et al. $(2000,2010)$ showed that the majority of CMEs reach a steady-state above about $10 \mathrm{R}_{\odot}$. On the other hand, our observations are restricted to the FOV of the SECCHI coronagraphs $\left(2.5-15 \mathrm{R}_{\odot}\right)$ and the 15 min cadence. This cadence diminishes the guaranteed observation range in the worst case to around $12 \mathrm{R}_{\odot}$, for a CME which expands along the POS with a speed of $2600 \mathrm{kms}^{-1}$. Sachdeva et al. (2017) showed that CME acceleration largely ends within $5 \mathrm{R}_{\odot}$ for fast events $\left(\geq 900 \mathrm{kms}^{-1}\right)$, and between 12 and $50 \mathrm{R} \odot$ for slow events $\left(<900 \mathrm{kms}^{-1}\right)$. The study of Liu et al. (2016) has even shown that the speed of slow CMEs is nearly invariant at heights above $20-30 \mathrm{R}_{\odot}$. Consequently, we can maximize the comparability of our events by choosing the latest guaranteed observation height of $12 \mathrm{R}_{\odot}$ as our reference height. In the following we present our GCS modelling:

In the first step, the six GCS parameters are iteratively adapted until a best visual fit of the data is achieved for both STEREO COR2 coronagraphs. Images from the SOHO/LASCO coronagraphs, positioned along the Sun-Earth line, are used to confirm the direction of the CME if it is seen as halo in both STEREO satellites. In the next step, we alter the fitting between the first and last COR2 image pairs for a few times. Generally, this approach is helpful to separate the main CME shape from unambiguous parts and shock features, which both are frequently observed in coronagraph white-light images of very intense events (Vourlidas et al. 2013; Vourlidas \& Ontiveros 2009). In the last step, the CME parameters are adapted to the reference height.

A sensitivity analysis by Thernisien et al. (2009) evaluates the average deviation of each parameter:

$$
\begin{gathered}
\Delta \phi=4.3^{\circ}, \Delta \theta=1.8^{\circ}, \Delta \alpha=+13^{\circ} /-7^{\circ}, \\
\Delta \kappa=+0.07 /-0.04, \Delta \gamma=22^{\circ}, \Delta h_{\text {apex }}=0.48 \mathrm{R}_{\odot}
\end{gathered}
$$

Equally, we have also estimated the errors of these parameters in our data set, based on the modelling result of the first and second author for a similar subset of 15 events. The averaged error $\Delta x_{0}$ of each GCS parameter $x$ yields:

$$
\begin{gathered}
\Delta \phi_{0}=5^{\circ}, \Delta \theta_{0}=5^{\circ}, \Delta \alpha_{0}=10^{\circ}, \\
\Delta \kappa_{0}=0.025, \Delta \gamma=30^{\circ}, \Delta h_{\text {apex }}=0.5 \mathrm{R}_{\odot}
\end{gathered}
$$

These values are in good agreement with the sensitivity analysis. Nevertheless, it has to be stated that the largest uncertainties for fitted GCS parameters arise from the misidentification of the CME shape, for example by the unintentional fitting of the CME shock or not separating the CME front from other coronal features. This might produce errors on a much larger scale which are difficult to quantify.

\subsection{Combined Geometrical Modelling and Mass Determination Technique}

We use the event of July 12, 2012 to illustrate our combined geometrical modelling and mass determination technique on a pair of SECCHI/COR2 images. On this day, the STEREO spacecraft were $125^{\circ}$ apart, which enables a reasonable geometrical modelling of the CME white-light shape. The described GCS fitting approach is used to derive the initial geometric parameters. Fig. 2 displays the projection of the geometrical grid in both SECCHI/COR2 images. The grid is created with the following fitting parameters for the reference height of $12 \mathrm{R}_{\odot}$ :

$$
\begin{gathered}
\phi=8^{\circ}, \theta=-12^{\circ}, \alpha=34.4^{\circ}, \\
\kappa=0.46, \gamma=90^{\circ}, \text { and } h_{\text {apex }}=15.6 \mathrm{R}_{\odot} .
\end{gathered}
$$

At this point, we create the mass images required for the directional mass determination. Savani et al. (2013) determined the CME mass density for a single event from SECCHI/COR2 images with this approach. In their study, the propagation direction in the corona was estimated by the propagation throughout the entire inner heliosphere via the fixed- $\phi$ J-map technique (Sheeley et al. 1999) from SECCHI/HI-1 and HI-2 observations. In our study, we can derive the CME propagation direction $\phi_{\mathrm{a}}$ and $\phi_{\mathrm{b}}$ in relation to the POS of STEREO A and B directly from our GCS modelling result. This step requires to transform the source region coordinates from HEE coordinate system by the respective STEREO satellite longitude. If one assumes a radial propagation of each CME from its active source re- 

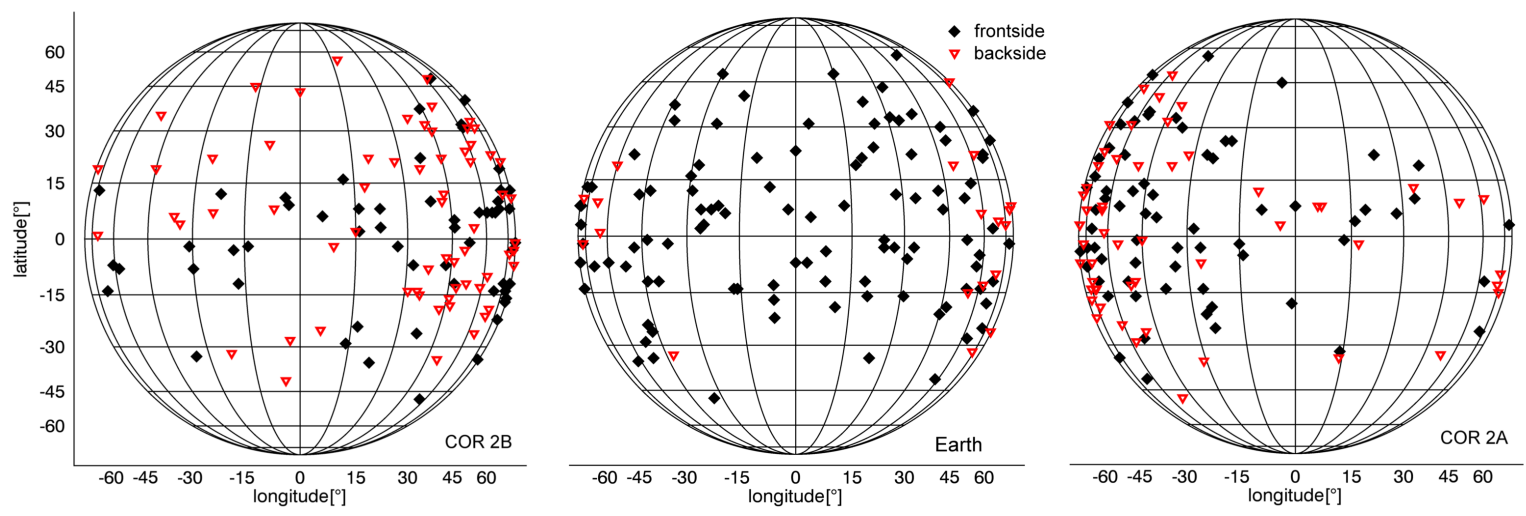

Figure 4. Source region distribution of all 122 KinCat events, estimated by GCS fitting and presented in the Earth-based Stonyhurst coordinate system (middle) as well as transposed to the perspectives of STEREO/SECCHI COR2A (right) and COR2B (left).
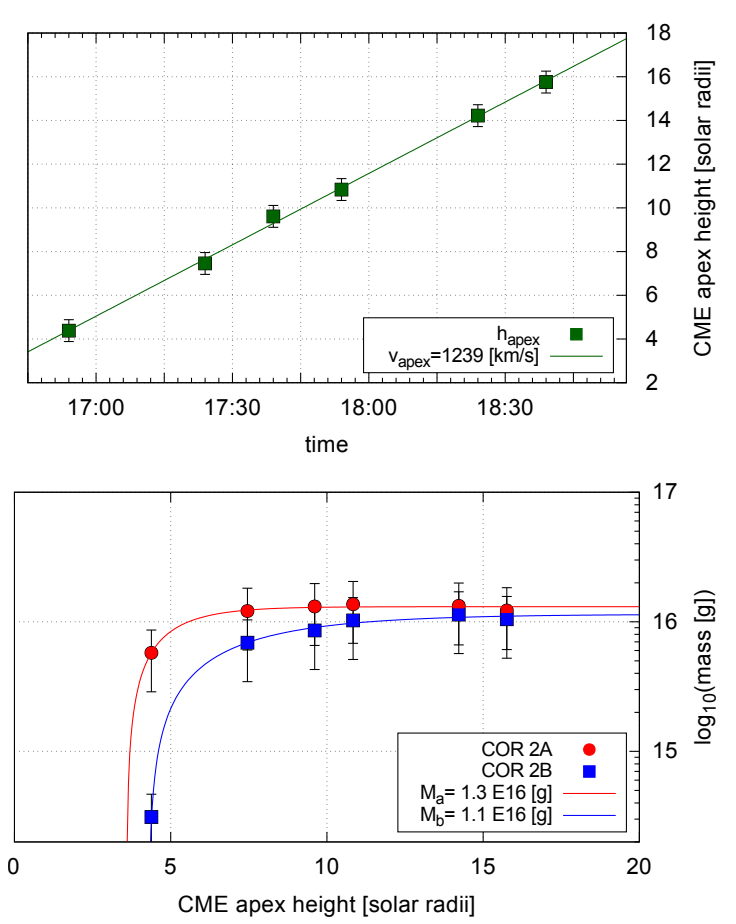

Figure 5. GCS fitting results of the July 12, 2012 event. Top: Height-time profile of the CME apex with the fitted apex speed $v_{\text {apex }}=1239 \mathrm{kms}^{-1}$ at $12 \mathrm{R}_{\odot}$. Bottom: Mass-height profile for both STEREO/SECCHI COR2 instruments with the extrapolated total masses $M_{\mathrm{a}}$ and $M_{\mathrm{b}}$.

gion (Reiner et al. 2003; Cremades \& Bothmer 2004; Kahler \& Vourlidas 2005), the modelled GCS flux rope source regions can be considered as approximations of the real source regions. For the calibration of the images, we consider the positions of the GCS modelled source regions and transpose them to the correspondent SECCHI/COR2 perspectives. In Fig. 4 we give an overview of the event distribution on the solar disk observed from Earth and from both STEREO/SECCHI COR2 perspectives, determined by our GCS modelling. We want to emphasise that the absence of central backside events along the Sun-Earth line is a result of the event selection requiring the association with a $\mathrm{SECCHI} / \mathrm{HI} \mathrm{CME}$

Events are classified based on their minimal angular propagation distance away from the observed POS. For $\phi<60^{\circ}$ we classify them as limb events and for $\phi \geq 60^{\circ}$ as disk events.

In the following, we determine the CME masses from the STEREO images. The applicability to SECCHI/COR2 images and cross-calibration with the $\mathrm{SOHO} / \mathrm{LASCO}$ mass images has been confirmed by Colaninno \& Vourlidas (2009). To determine a CME mass, the region containing the CMEs white-light emission, commonly termed region of interest (ROI), has to be defined. One advantage of our method is that we can benefit from our geometrical fitting results and define the ROI by the enclosed projection of the correspondent GCS grid as shown by Savani et al. (2013). The integration over the ROI in the mass images yields a mass value. For a sequence of mass images we apply the previously modelled GCS shape and expand it self-similarly to the correspondent height (Fig. 3). The fitted height values are used for the height-time profile in Fig. 5 (top), from which we derive the apex speed at $12 \mathrm{R}_{\odot}$. Based on the error propagation of the CME apex height, we derive that the height measurements correspond to a relative velocity error of $\Delta v / v=6 \%$. In Fig. 5 (bottom) the mass-height profile of the current example is displayed. V10 suggested that the mass and energy properties of CMEs are reaching a constant plateau above $\sim 10 \mathrm{R}_{\odot}$ if the POS mass determination approach is used. This is mostly, but not always the case for the directional method used in this study. Thus, we extrapolate the absolute CME mass $M_{0}$ from the massheight series. We use the fitting function from Colaninno \& Vourlidas (2009), which considers that CME masses can only be detected when a CME has overcome the occulter field of above $2.5 \mathrm{R}_{\odot}$ in COR2:

$$
M(h)=M_{0}(1-\exp (-h) / H)
$$

where $H$ is a fitted normalisation height and $M(h)$ the detected CME mass at the apex height $h$. 


\section{Results}

We present the statistical results of the GCS modelling and important parameter correlations to identify projection effects. This is followed by the presentation of the CME mass results, which are further analysed in terms of mass overestimations and causes. We demonstrate that the event selection criteria created an representative data-set which can reproduce known correlations to the monthly sunspot number (SSN). Further, the correlations to SSN have a useful application when it comes to comparing different data-sets. In the last part, we regard the mutual parameter correlation and analyse if the mass of a CME can be best estimated from the CME kinematic or the CME morphology.
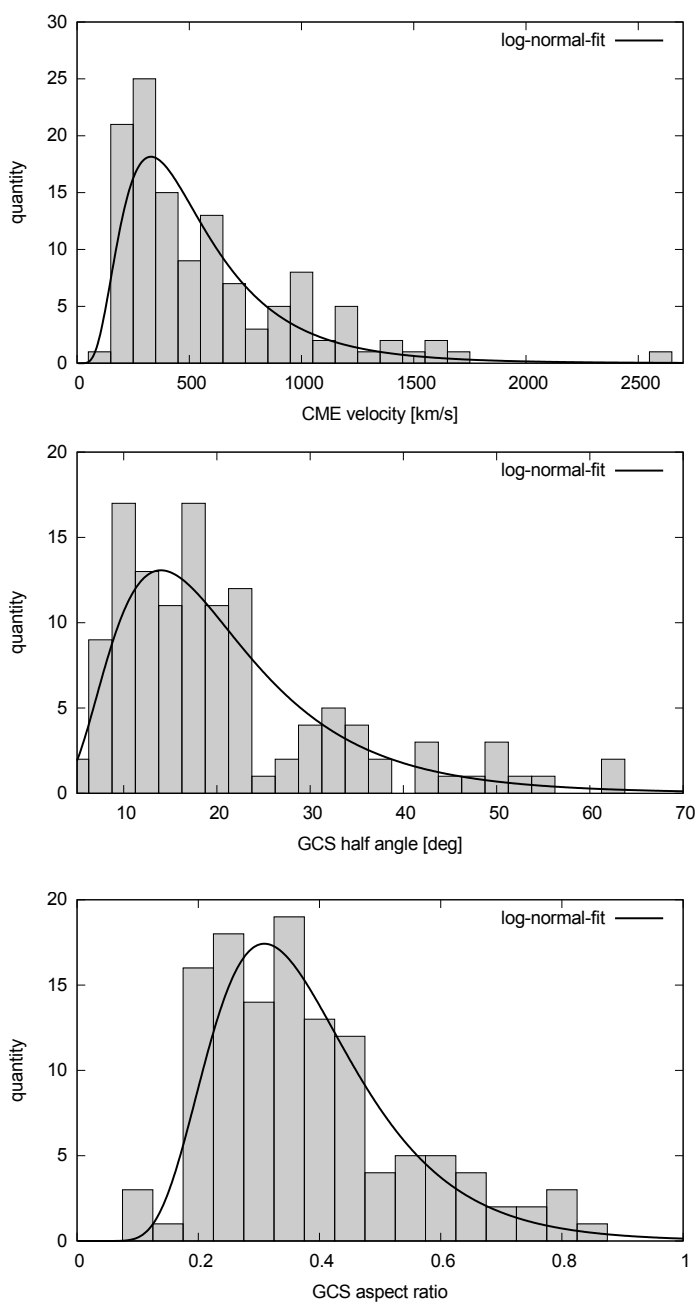

Figure 6. Distribution of the GCS modelling results at $12 R_{\odot}$ for the CME apex speed (top), the GCS half angle (middle) and the GCS aspect ratio (bottom).

\subsection{Morphology and Kinematics}

In the GCS model, the CME morphology is characterised by the aspect ratio and the half angle. We present distributions of the CME morphology together with the velocity distribution, which are derived from our data-set, in Fig. 6. All three distributions are basically reminiscent to a lognormal distribution, which is in this context also a typical parameter distribution in larger data-set (e.g. in V10). However, the CME velocities are closer to satisfy such a log-normal distribution than the two others. We obtain the averaged values of $\left\langle v_{\text {apex }}\right\rangle=629 \mathrm{kms}^{-1},\langle\kappa\rangle=0.405$ and $\langle\alpha\rangle=22.0^{\circ}$ for all 122 events.
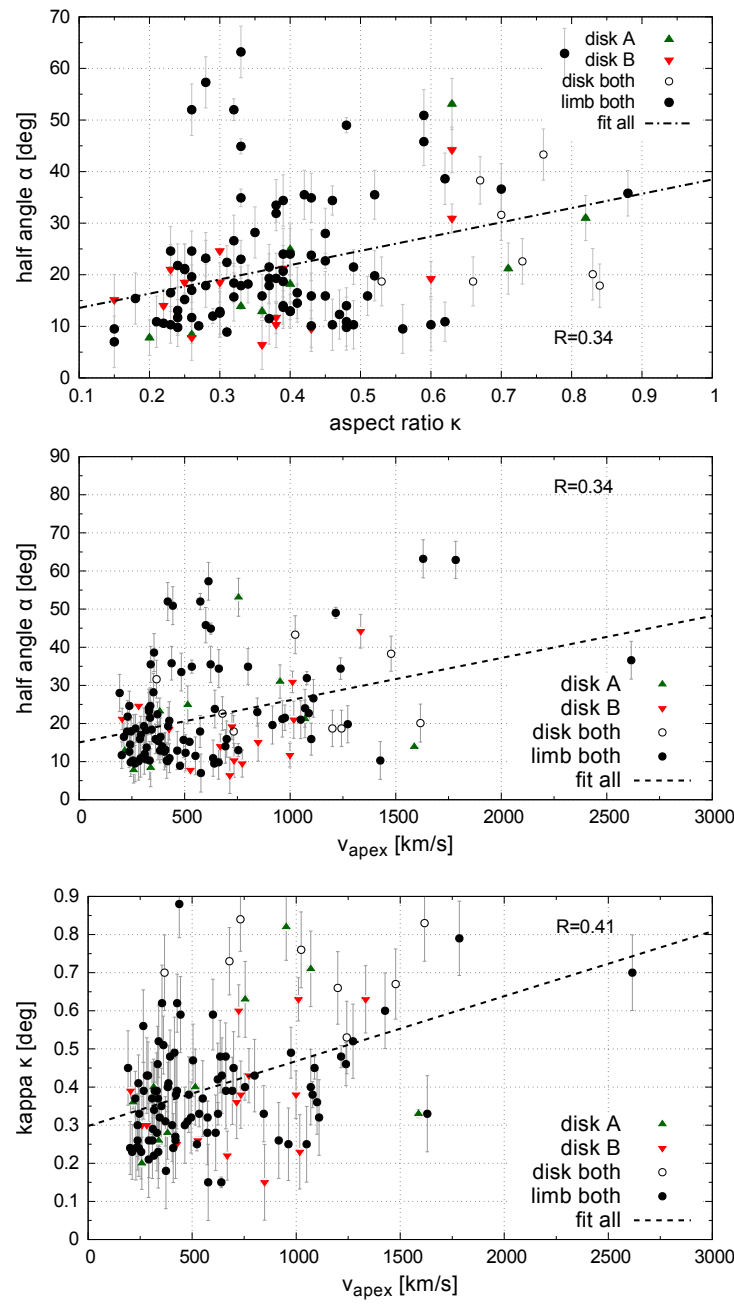

Figure 7. Individual results of GCS fitted aspect ratio $\kappa$ and half angle $\alpha$ derived for all 122 catalogue events. The events are classified by their limb or disk appearance in each STEREO/SECCHI COR2 instrument.

To investigate possible projection effects, we correlate all three parameters in Fig. 7 and further categorise each event based on its appearance as limb or disk event - accordingly to our previous definition. The parameter fits result in:

$$
\begin{aligned}
& \alpha=27.6( \pm 6.6) \kappa+10.8( \pm 2.8) \\
& \alpha=1.11( \pm 0.26) \times 10^{-2} v_{\text {apex }}+15.0( \pm 2.0) \\
& \kappa=1.7( \pm 0.3) \times 10^{-4} v_{\text {apex }}+0.30( \pm 0.02)
\end{aligned}
$$


with $\alpha$ in $[\mathrm{deg}], \kappa$ without dimension and $v_{\text {apex }}$ in $\left[\mathrm{kms}^{-1}\right]$.

We notice that in Fig. 7 (top) CMEs seen as disk event in both telescopes tend to have a much large $\kappa$ than the other events in the reference period. Also in Fig. 7 (bottom) all derived $\kappa$ values for disk events are above the linear fitting curve. Therefore, we investigate the occurrence of projection effects by comparing the morphology of the 8 disk event, which all occurred in 2011, to all 59 events of that year. We notice that they are comparable in terms of their half angle $\left(\alpha_{2011}=25^{\circ}\right.$ vs. $\left.\alpha_{\text {disk both }}=27^{\circ}\right)$ but not in terms of their aspect ratio $\left(\kappa_{2011}=0.45\right.$ vs. $\left.\kappa_{\text {disk both }}=0.71\right)$. It appears that $\kappa$ is significantly overestimated for disk events, which may be partly driven by the higher average speeds $\left(1050 \mathrm{kms}^{-1}\right)$ compared to the 2011 sample $\left(710 \mathrm{kms}^{-1}\right)$ in addition to projection effects.

\subsection{CME Mass Results}

In this section we show the results of our CME mass determination - averaged from both coronagraphs. In 3 out of 122 cases we have only obtained CME masses from COR $2 \mathrm{~A}$ as COR 2B images were intentionally avoided, due to overlapping with coronal features, such as secondary CMEs, solar event particles or coronal streamers. At first, we present the overall distribution of the logarithmic masses in Fig. 8. According to V10, the mass distribution of CMEs should generally follow a log-normal distribution which would result in a normal distribution of the logarithmic masses.

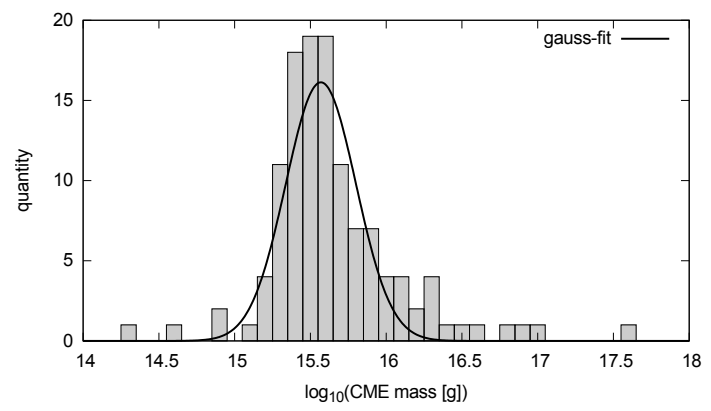

Figure 8. Distribution of the CME mass results in logarithmic units averaged from both SECCHI/COR2 measurements for 119 events and three single measurements.

In our results, we notice a departure from such a normal distribution towards larger mass values - a first indication for the mass overestimation of some events. To investigate this, we plot the logarithmic mass results of all 119 events which have been observed with both coronagraphs over the event dates in Fig.9. The events are classified by their limb or disk appearance in each STEREO/SECCHI COR2 instrument. Further, we add the minimal STEREO separation (MSS) angle $\sigma$ between both COR2 instruments.

Obviously, the highest mass values are detected between 2011 and 2012. Two points are noteworthy to mention at this phase, likely explaining these high values. First, the solar activity approaches a maximum around 2011-2013, which results in generally more massive CMEs (Vourlidas et al. 2010, 2017). Second, CMEs tend to appear with redundant projection shapes in both COR2 telescopes since

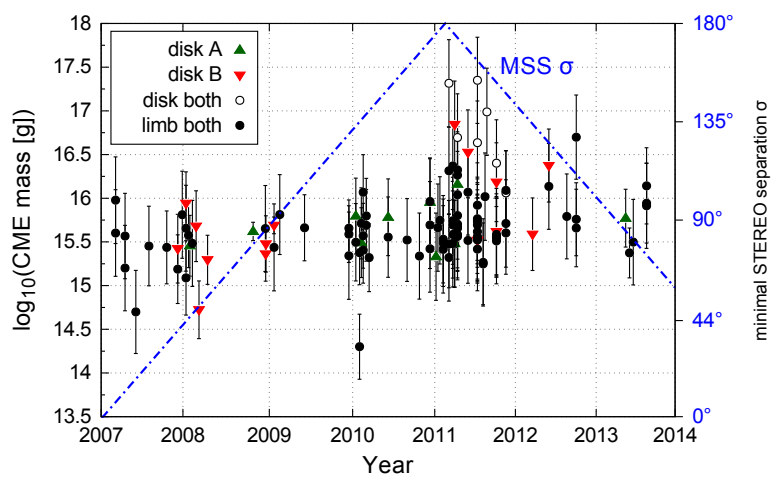

Figure 9. CME mass in logarithmic units for 119 events averaged from both coronagraphs and classified by their limb or disk appearance in each COR2 instrument together with STEREO geometry, which is characterised by the minimal STEREO separation (MSS) angle $\sigma$ (dashed).

the spacecraft are in opposition. This configuration occurs when the MSS angle $\sigma$ is close to $0^{\circ}$ or $180^{\circ}$. As several of the more massive entries in Fig. 9 are from CMEs seen as disk events in both telescopes, this suggests an additional influence of projection effects leading to mass overestimations in such cases.

\subsection{Mass Overestimations}
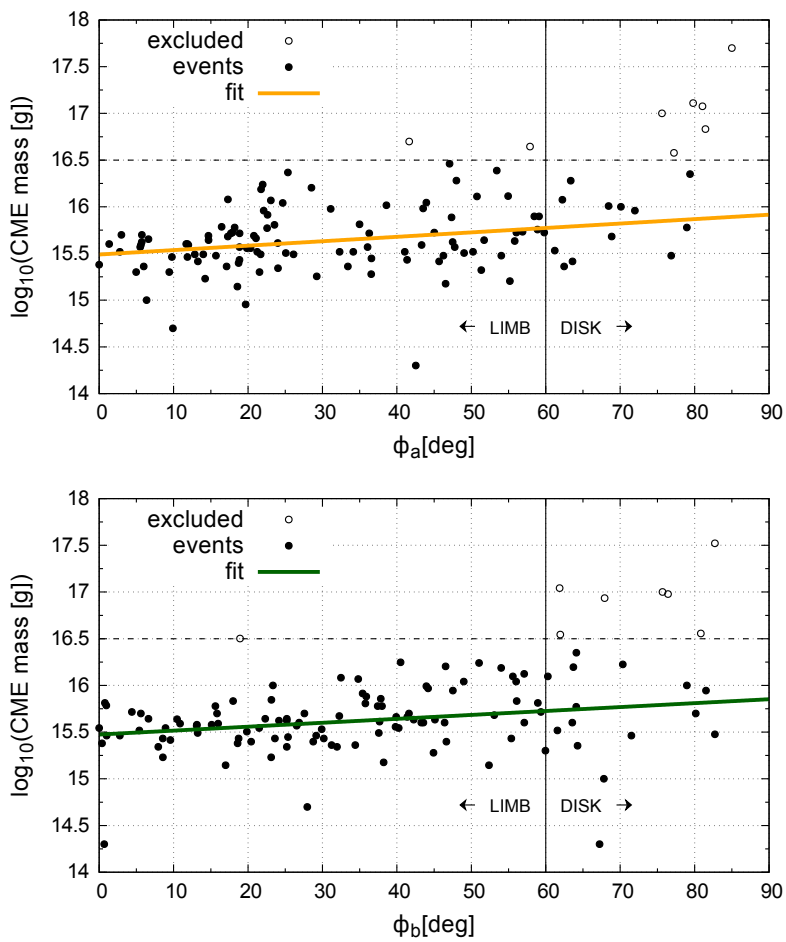

Figure 10. Logarithmic CME masses for 122 events are measured in COR 2A (top) and 119 events in COR 2B (bottom) against the angular distance $\phi$ of their propagation direction away from the POS. 
Since we do not know the 3-dimensional electron distribution within a specific CME, we assume that it is equivalent to its 2-dimensional projection, as stated in Section 2.2.1. However, the 2-dimensional projection changes with the observed propagation direction and as consequence also the determined mass values. We compare the propagation direction away from the POS for each coronagraph in Fig. 10 with the determined mass values to investigate if the CME masses of disk events are overestimated by such a geometrical effect. For a single spacecraft study with the SOHO/LASCO coronagraph, covering over 7000 CME nonhalo events, V10 found that the majority of CMEs masses lie in the range of $10^{13}-10^{16} \mathrm{~g}$. Therefore, CME masses bigger than $10^{16.5} \mathrm{~g}$ are excluded from our further analysis, due to an expected mass overestimation. This applies to eight events. We further notice that the statistical mass overestimation of disk events at angle of $\phi=80^{\circ}$ away from the solar centre reaches an average factor of three. At the same time we see a clustering of the CME masses of disk events with a likely additional overestimation of about a factor of ten or more, which is way above the maximum error of a factor of five as derived by V10. Therefore, the projection effects on the CME alone are insufficient to explain such large mass values.
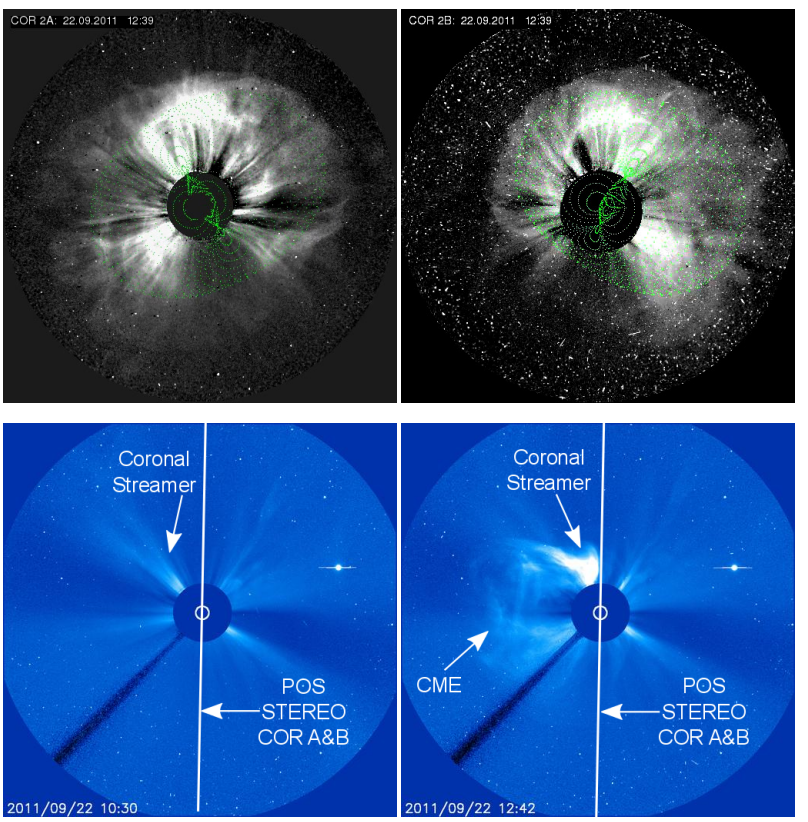

Figure 11. (Top:)GCS modelling of the CME event on September 22, 2011, observed with the STEREO COR 2A (left) and STEREO COR 2B (right), which are positioned at $\phi_{b}=-97^{\circ}$ and $\phi_{a}=104^{\circ}$ in HEE. (Bottom:) The same event observed with the LASCO C3 coronagraph. During the eruption, a preexisting coronal streamer increases in brightness and is deflected towards solar north pole - close to the POS of both COR2 coronagraphs.

A possible explanation for the usually high mass values could arise from additional brightness contributions of other coronal features. We present an illustrative example for such a case with the CME event on September 22, 2011. This CME is seen as disk event in the opposing STEREO coronagraphs (Fig. 11, top). At least two intense streamers appear within our ROI, which is defined by the green GCS fit, and contribute to the overall brightness. Our mass determination yields a value of $3 \times 10^{17} \mathrm{~g}$, the highest mass value of the KinCat. We compare our obtained mass values of this event to the mass value in the $\mathrm{SOHO} / \mathrm{LASCO}$ CME Catalogue. The SOHO/LASCO C3 coronagraph is positioned at $\phi=0$ and observes the event perpendicular to both COR2 coronagraphs. The C3 images (Fig. 11, bottom) suggest that a northern pre-existing streamer increases in brightness and is deflected towards the POS of COR2 coronagraphs during the eruption. Obviously, such streamers can not be removed with a difference imaging approach. For comparison - from the LASCO perspective, the northern streamer is largely outside of the ROI used in the mass calculation and contributes less to the overall brightness. The reported mass for this event is $2.1 \times 10^{16} \mathrm{~g}$, but with some uncertainty due to the presence of a shock. However, the northern streamer can not be excluded from the COR2 ROI, which results in a total mass increase of 1.4 orders of magnitude.

\subsection{CME Correlations}

Before we regard the individual parameter correlation, we like to proof that our data-set can reproduce the known and more basic correlations to the solar cycle. Ivanov \& Obridko (2001) have shown that the cycle variation of the average CME width and average CME velocity are associated to the cycle variation of the large-scale solar magnetic field for solar cycle 22 . It was further stated, that fast CMEs make the primary contributions in these statistics. As far as the CME mass is concerned, V10 have shown their dependence to the activity of solar cycle 23. By investigating solar cycle 24, Wang \& Colaninno (2014) also stated that fast, wide and massive CMEs undergo a stronger solar cycle correlation. Our data-set is biased towards bright and generally fast events, we therefore expect them to reflect the correlation to the solar cycle activity very well. In Fig. 12, we demonstrate this by using annually averaged

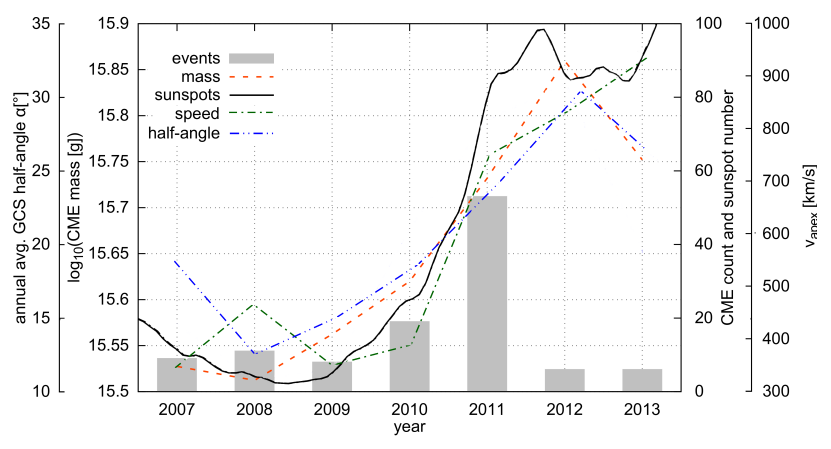

Figure 12. Annual evolution of the average half angle, average CME speed for 122 events and the CME mass for 114 events of the KinCat. CME masses above $10^{16.5} \mathrm{~g}$ are excluded. Further, the annual event count and the total 13-month smoothed monthly sunspot numbers are displayed. The sunspot numbers are provided by WDC-SILSO, Royal Observatory of Belgium, Brussels.

values of half angles, the apex velocities and CME masses of our data-set together with the activity of solar cycle 24 , 
represented by SSN. For the mass analysis, we have ap-

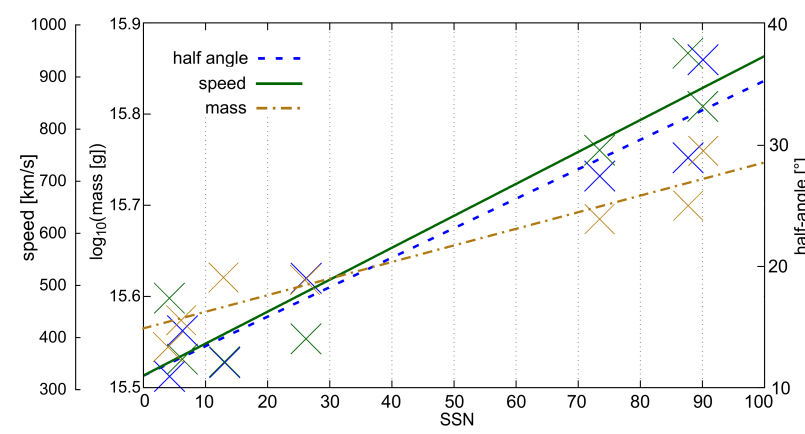

Figure 13. Annual average of the GCS half angle and CME speed (122 KinCat events) as well as of the CME mass (114 KinCat events) over the total 13-month smoothed monthly sunspot numbers. CME masses above $10^{16.5} \mathrm{~g}$ are excluded. The sunspot numbers are provided by WDC-SILSO, Royal Observatory of Belgium, Brussels.
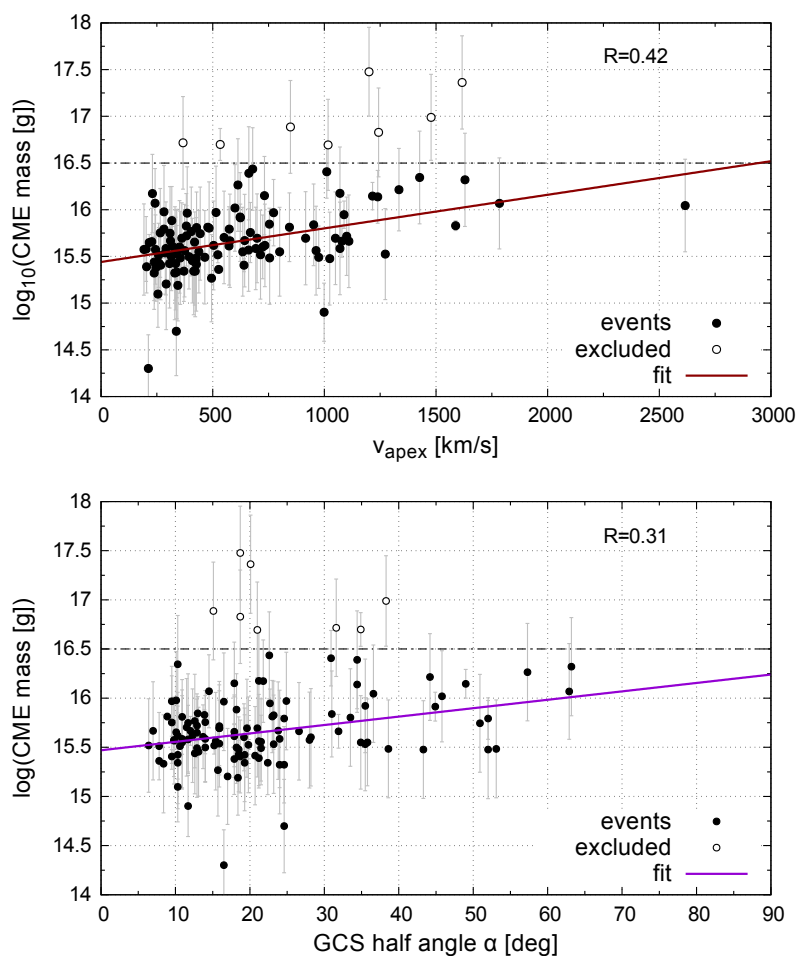

Figure 14. Averaged CME masses for all 122 events from the KinCat against (left) the CME apex speed and (right) the CME half angle. CME masses above $10^{16.5} \mathrm{~g}$ are excluded for the fitting.

plied the same upper CME mass limits as before to reduce influence of some questionable mass results. We derived the following linear correlations between the annual average of the monthly sunspot number and the annual averages of the CME mass, speed and GCS half angle from Fig. 13 :

$$
\begin{aligned}
\log _{10}(M) & =15.51( \pm 0.02)+0.0032( \pm 0.0004) S S N \\
v_{\text {apex }} & =328.3( \pm 48.6)+6.1( \pm 0.9) S S N \\
\alpha & =14.7( \pm 1.3)+0.15( \pm 0.02) S S N
\end{aligned}
$$

with $\alpha$ in [deg], $v_{\text {apex }}$ in $\left[\mathrm{kms}^{-1}\right]$ and $M$ in [g]. Such connections of CME event properties to the SSN may also be useful for the comparison of different CME data-sets. All three parameters largely correlate as expected with the SSN. Only in 2013 we see that the average half angle and the average CME mass descends in contrast to the CME speed. This is likely a result of the different event selection criteria for the years 2012-2013. Also, the low event count during a very active solar period makes the results of this phase likely less significant. However, these statistics are in agreement with the (Vourlidas et al. 2017) statistics, which are based on a much larger sample of COR2 events. Our higher speed average is likely a result caused by our event selection towards brighter and generally faster CMEs.

We now continue by presuming a mutual dependency of the CME speed and width to the CME mass. Since the latter is usually the most difficult to measure in near realtime, the outcome could lead to useful applications in CME forecasts. In Fig. 14 we plot the average CME masses for all 122 events from the KinCat against the CME apex speed and the CME half angle. We have applied the same upper CME mass limits as before. The two linear parameter fits result in:

$$
\begin{aligned}
& \log _{10}(M)=3.4( \pm 0.6) \times 10^{-4} v_{\text {apex }}+15.479( \pm 0.044) \\
& \log _{10}(M)=8.71( \pm 1.82) \times 10^{-3} \alpha+15.380( \pm 0.065)
\end{aligned}
$$

with $\alpha$ in [deg], $v_{\text {apex }}$ in $\left[\mathrm{kms}^{-1}\right]$ and $M$ in $[\mathrm{g}]$. As the majority of the excluded events with masses above $10^{16.5} \mathrm{~g}$ are also identical with the 8 disk events of our data-set, it is noteworthy, that we have at the same time excluded possible large uncertainties in the velocity and the half angle values caused by projection effects.

\section{Discussion}

First of all, we like to point out the advantages of the dataset created by our combined method. It provides the two most important CME parameters in the context of CME arrival analysis with the DBM - the CME mass and the CME speed in the direction of Earth. As the latter, is only provided indirectly, we want to explain how to derive this value from our data-set. In Fig. 15 we expand the fitted GCS shape self-similarly in the Heliocentric Earth Ecliptic (HEE) coordinate system to $1 \mathrm{AU}$, which allows to analyse if the CME is Earth-directed and which part of it will potentially impact Earth. If only the slower flank and not the CME apex is directed towards Earth, the apex speed can be a very inaccurate proxy for the Earth-directed speed. Instead, the speed component towards Earth can be derived from the data-set by

$$
v_{\text {earth }}=\left(h_{\text {earth }} / h_{\text {apex }}\right) v_{\text {apex }},
$$

where $h_{\text {apex }}$ is the apex height, $h_{\text {earth }}$ is the height in Earth-direction and $v_{\text {apex }}$ is the initial apex speed. CME propagation models, as the DBM, can now be applied and 
their results can be compared to in-situ measured CME signatures at L1.

\subsection{CME Morphology}

We have analysed the GCS morphology, represented by the values of $\kappa$ and $\alpha$, of our modelling results. In Section 3.1 we have shown that the results are consistent in the case that events are seen as pure limb event in both coronagraphs or as a combination of disk and limb event. On the other hand, we noticed an significant overestimation of $\kappa$ if the event is seen as disk events in both coronagraphs. In such cases, the shape projection of disk events appears as ellipse, where the semi-minor axis is defined by $\kappa$ and semi-major axis by $\kappa$ and $\alpha$. We want to emphasise that the fitting accuracy of $\kappa$ and $\alpha$ also depends on the rotation of the GCS model and generally favours one or the other. The determination of the GCS model rotation can be extremely difficult for disk events, especially without considerations of the orientation of the source region. For that reasons, such overestimations of the CME morphology might also affect the determined $\alpha$ values. This can possibly account for some of the spread of data from the fitting line.

There are three possible explanations for the general trend of the larger measured morphology of disk events. First, if an event appears as disk event in both coronagraphs, the instrument separation needs to be very small or close to $180^{\circ}$. In this case the perspectives are nearly redundant and the projection effect are converging to the known effects for observations from only one vantage point. Second, for larger $\kappa$ the projected GCS shapes appear more circular, rather than elliptical. The fact that all eight disk events are concerned, let us conclude that this a more general phenomenon resulting from projection effects, due to which modellers tend to overestimate $\kappa$. This overestimation generally leads to a more circular projected CME shape, rather than elliptic, which may be a hint, that disk events heavily favour unintentional additional fitting of CME shock features, which encloses the regular CME shape. It has to be evaluated how the knowledge of this effect and the shown $\kappa$ to $\alpha$ correlation in Equation 11 can contribute to a more accurate fitting of disk events. Last, the presence of additional coronal features might also lead to an overestimation of the morphology. Since, these results are obtained under the presence of a very active corona,

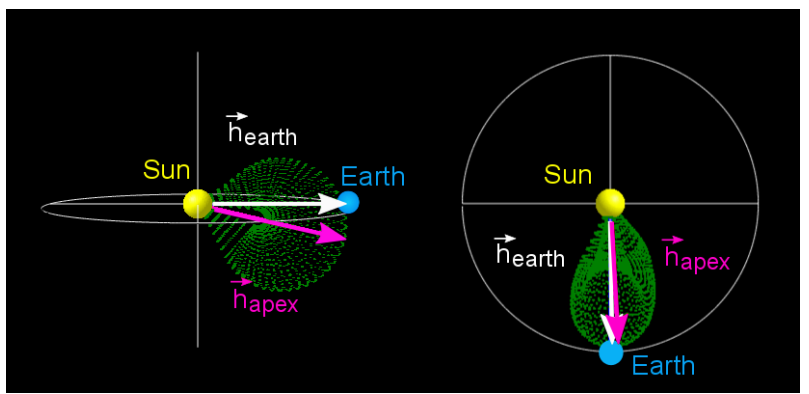

Figure 15. GCS-shape of the July 12, 2012 event propagated self-similarly to $1 \mathrm{AU}$ in the HEE coordinate system. The height of GCS front in the main axis and in Earth-direction are included for illustration. the influence of coronal features could partially contribute to this results.

\subsection{CME Velocity}

We further compared the GCS modelling result for disk and limb events to their velocity in Section 3.1. It was frequently discussed that projection effects of halo events generally lead to an overestimation of the CME morphology and therefore to an underestimation of the CME speed (Gopalswamy et al. 2010). In the work Yashiro et al. (2004), which is based on projected CME speeds, it was reported that fast CMEs tend to be larger. However, the correlation was described as very weak. Generally, the determined speeds of non-deprojection disk events is on average 25-50\% slower as their deprojected speeds (Vršnak et al. 2007b). Shen et al. (2013b) compared projected and deprojected CME speeds. They have shown that most full halo CMEs, originating within 45 degree away from the SunEarth line and possessing a projected speed slower than $900 \mathrm{kms}^{-1}$ suffer from large projection effect in contrast to faster ones.

In this work we regard deprojected CME speeds and the shown velocity distribution in Fig. 6 seems to follow a typical log-normal distribution. We have analysed the relation between the CME velocity and the CME morphology. Our data has shown that the CME speed correlates at least moderately with the deprojected morphology $(R=0.34$ for $\alpha$ and $R=0.41$ for $\kappa$ ). Even with the overestimation of the morphology, we have not noticed a significant velocity underestimation of disk events, which would be the consequence of known projection effects for observations from only one vantage point. Likely, accurate stereoscopic velocity measurements require a smaller separation of both observing instruments than accurate measurements of the morphology.

\subsection{Mass Determination}

The mass results have been presented in Section 3.2. We already mentioned, that V10 found that the majority of CMEs masses lie in the range of $10^{13}-10^{16} \mathrm{~g}$, and for that reason consider masses higher than $10^{16.5} \mathrm{~g}$ as suspicious. We have detected significantly higher masses for eight CMEs - five of them were seen as disk events in both COR2 instruments and two others as disk events in COR2 B. We investigated the systematical overestimation of CME masses, based on our directional mass determination method in Section 3.3. V10 stated that this method improves the accuracy of the mass measurements relative to the sky plane assumption to about $\phi=60^{\circ}$, but decreases fast beyond that angle. At $\phi=80^{\circ}$, this mass overestimation reaches about factor five, based on hypothetical CME width of $2 \alpha=60^{\circ}$.

We have presented the disk-limb mass distribution for both COR2 coronagraphs and estimated the mass overestimation based on the overall geometry. The statistical mass overestimation of disk events at $\phi=80^{\circ}$ reaches an average factor of three for the mass range of $10^{15}-10^{16.5} \mathrm{~g}$. If the eight events with higher mass values are also included into the analysis, the overestimation reaches a factor of six. We conclude that geometrical effects on the CME alone can not fully explain the largest mass values of our data-set. 
We have shown an example for another relevant source of mass overestimation due to the presence of a coronal streamer. In this case the mass overestimation of the directional mass determination reached a factor of fourteen. We have also discussed, that such sources of mass overestimation are most likely for disk events during phases of high solar activity. The visual inspection of the eight overestimated events with the further help of the LASCO C3 coronagraph, positioned close to Earth, confirmed that in seven cases the additional mass results from coronal streamers (see example in Fig 11, Section 3.3) and in one case from the occurrence of another CME.

One can notice that an overestimation and misinterpretation of the GCS shape was caused by streamers in two cases and by a CME shock front in one case. We identified a relevant explanation for the large mass values of our disk events with the presence and brightness enhancement of overlapping coronal streamers. From this example event we deduce that overlapping structures can be a significant source of mass overestimation. Such overlapping structures occur much more likely during the solar maximum and they are also much more likely present for disk events, as these exhibit larger areas in the observers FOV compared to limb events. For that reason, we suggest to estimate the CME mass of disk events based on their relation to other CME parameter.

\subsection{Empirical Correlations}

In Section 3.4 we confirmed that the kinematics, morphologies and masses of our observed CMEs correlate very well with the solar activity cycle. Hence, the question arose if the kinematics or morphology of a CME could be used to estimate the CME mass. We presented both correlations and have shown that the mass-velocity correlation coefficients of Equation 8 is slightly superior $(R=0.41)$ to the one of the mass-half angle correlation in Equation $9(R=0.31)$. Moreover, we have discussed that errors of the CME speed measurements are smaller compared to the measurement of the CME morphology. Of course both are most reliable if the CME is seen as limb event in at least one perspective. Thus, we recommend to use Equation 8 when it comes to empirical estimations of the CME mass. The advantage of this result is that speed measurements can be used to derive the other CME properties. Once applied to a $\mathrm{CME}$ propagation model, as the DBM, they result in more accurate first estimations of the terrestrial arrival of intense CMEs. These results might also be valuable for setting observational constraints in space weather prediction models such as ENLIL (Odstrcil et al. 2004) and empirical models for predicting the parameters at L1 (Austin \& Savani 2018).

\section{Summary - Conclusion}

The objective of this work is to present a combined method of geometrical modelling and CME white-light mass determination. The method has been applied to a set of 122 intense events observed with STEREO COR2 roughly spanning over solar cycle 24 . We determined the threedimensional flux rope structure of these CMEs with the Graduated Cylindrical Shell (GCS) model. We further determined the mass content of each event based on the di- rectional mass determination approach under the consideration of the CMEs propagation direction. All determined kinematic, geometric and mass parameters of the 122 events are gathered in the "KinCat" online catalogue. All results are referenced to a fixed apex height of $12 \mathrm{R}_{\odot}$, to faciliate comparison among all events. Moreover, the annual average of all derived parameters are associated to the SSN to make them comparable to other studies. To quantify the influence of the geometry in the fitting results, we have separated our events by their appearance as limb or disk events in the COR2 coronagraphs. Within this study, we have analysed the applicability of the presented method to disk events. Further, we investigated if the CME mass can be empirically estimated from its connection to the CME kinematic. From the analysis of the catalogue parameters, we obtained the following results:

1. The CME morphology of disk events can lead to an overestimation of the flux rope width up to a factor of two - at least if coronal features are present.

2. The average morphology of the CMEs within our dataset can be described with the GCS half angle $\alpha$ and the GCS aspect ratio $\kappa$ via:

$$
\alpha=27.6 \kappa+10.8
$$

3. The obtained CME speeds of disk events are less affected by projection effects than the CME morphology. The latter can be estimated for disk events from the CME speed via:

$$
\begin{aligned}
& \alpha=1.11 \times 10^{-2} v_{\text {apex }}+15.0 \\
& \kappa=1.7 \times 10^{-4} v_{\text {apex }}+0.30
\end{aligned}
$$

4. Projection effects lead to an average mass overestimation for our applied mass determination approach. The overestimation reaches a factor of three for disk events emerging at an angular distance of $\phi=80^{\circ}$ away from the POS.

5. Mass overestimations of a factor of ten and more can result from overlapping features. Such effects are more likely present in disk events because they tend to occupy a large range of position angles due to the observed geometry.

6 . Finally, we propose that the following empirical correlation between the CME masses and the CME speeds is most suited to estimate the mass of intense CMEs from coronagraph images:

$$
\log _{10}(M)=3.4 \times 10^{-4} v_{\text {apex }}+15.479 .
$$

We conclude that for stereoscopic measurements of disk events, the measurements of initial CME speed are the most reliable ones and can be used to derive the CME morphology and mass to make them quickly applicable in space weather forecasts. However, for scientific purposes, such derived values should be treated with caution.

Acknowledgements. The work in this paper was funded under the HELCATS project by the EU FP7 program. A.Vourlidas is supported by NASA grants NNX16AH70G and NNX17AC47G. Volker Bothmer acknowledges support of the CGAUSS (Coronagraphic German and US Solar Probe Plus Survey) project for WISPR on NASA PSP by the German Space Agency DLR under grant 50 OL 1601 
and the support of the project Stereo/Corona by the German Bundesministerium fur Bildung und Forschung through the deutsche Zentrum fur Luft-und Raumfahrt e.V. (DLR, German Space 59 Agency). Stereo/Corona was a science and hardware contribution to the optical image package SECCHI, developed for the NASA STEREO mission. The STEREO/SECCHI data used for this study are prepared by an international consortium of NASA Goddard Space Flight Center (USA), Lockheed Martin Solar and Astrophysics Lab (USA), Naval Research Laboratory (USA), Rutherford Appleton Laboratory (UK), University of Birmingham (UK), Max-Planck-Institut für Sonnensystemforschung (Germany), Institut d'Optique Thèorique et Appliquèe (France), Institut d'Astrophysique Spatiale (France) and Centre Spatiale de Liège (Belgium). We further acknowledge close collaborations with the U.S. Naval Research Laboratory (NRL), Dr. Russ A. Howard (PI of STEREO/SECCHI), Dr. Arnaud Thernisien and Dr. Jackie Davies from the Rutherford Appleton Laboratory (RAL), Dr. Eckhard Bosman, Malte Venzmer and Johannes Hinrichs from University of Göttingen (UGOE).

\section{References}

Austin, H. J. \& Savani, N. P. 2018, Weather, n/a

Bein, B. M., Temmer, M., Vourlidas, A., Veronig, A. M., \& Utz, D. 2013, Astrophys. J., 768, 31

Bosman, E. \& Bothmer, V. 2012, in EGU General Assembly Conference Abstracts, Vol. 14, EGU General Assembly Conference Abstracts, ed. A. Abbasi \& N. Giesen, 11632

Bosman, E., Bothmer, V., Nisticò, G., et al. 2012, Solar Physics, 281 167

Burkepile, J. T., Hundhausen, A. J., Stanger, A. L., St. Cyr, O. C. \& Seiden, J. A. 2004, Journal of Geophysical Research (Space Physics), 109, A03103

Burlaga, L. F., Behannon, K. W., \& Klein, L. W. 1987, J. Geophys. Res., 92, 5725

Chen, J., Howard, R. A., Brueckner, G. E., et al. 1997, Astrophys. J. Lett., 490, L191

Cherry, N. 2002, Natural Hazards: Journal of the International Society for the Prevention and Mitigation of Natural Hazards, 26 279

Colaninno, R. C. \& Vourlidas, A. 2009, Astrophys. J., 698, 852

Cremades, H. \& Bothmer, V. 2004, Astron. Astrophys.,422, 307

Davies, J. A., Perry, C. H., Trines, R. M. G. M., et al. 2013, Astrophys. J., 777,167

de Koning, C. A. 2017, Astrophys. J., 844, 61

Gopalswamy, N. 2013, in Astronomical Society of India Conference Series, Vol. 10, Astronomical Society of India Conference Series

Gopalswamy, N., Yashiro, S., Michalek, G., et al. 2010, Sun and Geosphere, 5,7

Gosling, J. T., McComas, D. J., Phillips, J. L., \& Bame, S. J. 1991 J. Geophys. Res., 96, 7831

Harrison, R., Davies, J., Perry, C., et al. 2016, in EGU General Assembly Conference Abstracts, Vol. 18, EGU General Assembly Conference Abstracts, EPSC2016-10220

Howard, R. A., Michels, D. J., Sheeley, Jr., N. R., \& Koomen, M. J. 1982, Astrophys. J., Lett., 263, L101

Howard, R. A., Moses, J. D., Vourlidas, A., et al. 2008, Space Science Reviews, 136, 67

Ivanov, E. V. \& Obridko, V. N. 2001, Solar Physics, 198, 179

Kahler, S. W. \& Vourlidas, A. 2005, Journal of Geophysical Research (Space Physics), 110, A12S01

Kaiser, M. L., Kucera, T. A., Davila, J. M., et al. 2008, Space Science Reviews, 136,5

Kilpua, E., Möstl, C., Bothmer, V., et al. 2017, in EGU Genera Assembly Conference Abstracts, Vol. 19, EGU General Assembly Conference Abstracts, 9051
Kwon, R.-Y., Zhang, J., \& Vourlidas, A. 2015, Astrophys. J., Lett., 799, L29

Lee, H., Moon, Y.-J., Na, H., \& Jang, S. 2014, in American Astronomical Society Meeting Abstracts, Vol. 224, American Astronomical Society Meeting Abstracts \#224, 218.34

Liewer, P. C., Hall, J. R., Howard, R. A., et al. 2011, Journal of Atmospheric and Solar-Terrestrial Physics, 73, 1173

Liu, Y., Davies, J. A., Luhmann, J. G., et al. 2010a, Astrophys. J., Lett., $710, \mathrm{~L} 82$

Liu, Y., Thernisien, A., Luhmann, J. G., et al. 2010b, Astrophys. J.,

Liu, Y. D., Hu, H., Wang, C., et al. 2016, Astrophys. J., Suppl. Ser., 222,23

Mays, M. L., Taktakishvili, A., Pulkkinen, A., et al. 2015, Solar Physics, 290, 1775

Odstrcil, D., Pizzo, V. J, Linker, J. A, et al, 2004, Journal of Atmospheric and Solar-Terrestrial Physics, 66, 1311

Odstrčil, D. \& Pizzo, V. J. 1999, J. Geophys. Res., 104, 493

Pulkkinen, A., Lindahl, S., Viljanen, A., \& Pirjola, R. 2005, Space Weather, 3, S08C03

Reiner, M. J., Vourlidas, A., Cyr, O. C. S., et al. 2003, Astrophys. J., 590,533

Sachdeva, N., Subramanian, P., Vourlidas, A., \& Bothmer, V. 2017, Solar Physics, 292, 118

Savani, N. P., Owens, M. J., Rouillard, A. P., et al. 2011a, Astrophys. J., 731, 109

Savani, N. P., Owens, M. J., Rouillard, A. P., et al. 2011b, Astrophys. J., 732,117

Savani, N. P., Vourlidas, A., Pulkkinen, A., et al. 2013, Space Weather, 11, 245

Sheeley, N. R., Walters, J. H., Wang, Y.-M., \& Howard, R. A. 1999, J. Geophys. Res., 104, 24739

Shen, C., Wang, Y., Pan, Z., et al. 2013a, Journal of Geophysical Research (Space Physics), 118, 6858

Shen, C., Wang, Y., Pan, Z., et al. 2013b, Journal of Geophysical Research (Space Physics), 118, 6858

Temmer, M., Preiss, S., \& Veronig, A. M. 2009, Solar Physics, 256, 183

Thernisien, A., Vourlidas, A., \& Howard, R. A. 2009, Solar Physics, 256,111

Thernisien, A. F. R., Howard, R. A., \& Vourlidas, A. 2006, Astrophys. J., 652, 763

Thomson, A. W. P., Dawson, E. B., \& Reay, S. J. 2011, Space Weather, 9, S10001

Tsurutani, B. T., Smith, E. J., Gonzalez, W. D., Tang, F., \& Akasofu, S. I. 1988, J. Geophys. Res., 93, 8519

Vourlidas, A., Balmaceda, L. A., Stenborg, G., \& Dal Lago, A. 2017, Astrophys. J., 838, 141

Vourlidas, A. \& Howard, R. A. 2006, Astrophys. J., 642, 1216

Vourlidas, A., Howard, R. A., Esfandiari, E., et al. 2010, Astrophys. J., 722,1522

Vourlidas, A., Lynch, B. J., Howard, R. A., \& Li, Y. 2013, Solar Physics, 284, 179

Vourlidas, A. \& Ontiveros, V. 2009, in American Institute of Physics Conference Series, Vol. 1183, American Institute of Physics Conference Series, ed. X. Ao \& G. Z. R. Burrows, 139-146

Vourlidas, A., Subramanian, P., Dere, K. P., \& Howard, R. A. 2000, Astrophys. J., 534, 456

Vršnak, B., Sudar, D., Ruždjak, D., \& Žic, T. 2007a, Astron. Astrophys., 469, 339

Vršnak, B., Sudar, D., Ruždjak, D., \& Žic, T. 2007b, Astron. Astrophys., 469, 339

Vršnak, B., Zic, T, Vrbanec, D, et al. 2013, Solar Physics, 285, 295

Wang, Y.-M. \& Colaninno, R. 2014, Astrophys. J., Lett., 784, L27

Yashiro, S., Gopalswamy, N., Michalek, G., et al. 2004, Journal of Geophysical Research (Space Physics), 109, A07105 


\subsection{Mass Overestimation from Coronal Streamers}

The previous section has revealed that coronal streamers can be a huge source of CME mass overestimation. In the following, this fact shall be demonstrated in more detail. To this end, two sample events of the KinCat are presented, for which the CME mass determination suffers from this effect. The coronagraph images of STEREO, in which these events appear as halos, are compared to the coronagraph images of $\mathrm{SOHO}$, which is positioned perpendicular to the STEREO images, so that it observes the events as limb events.

Sample \#11 - Helcats Event \#95

Sample event \#11 occurred on 01-10-2011. To this date both STEREO spacecraft have a separation of $167^{\circ}$. The GCS fit of this event is presented in Fig. 8.1 and the fitting parameters in Table 8.1. The orientation of the GCS grid in relation to earth is presented in Fig. 8.2 and the SOHO C3 observations in Fig. 8.1. The CME is directed towards COR 2B and posses an apex speed of $1478 \mathrm{~km} / \mathrm{s}$. The mass determination in both COR 2 images has been affected by the northern streamer. This streamer could be excluded in the ROI of LASCO C3 images. Therefore the measured mass in C3 is factor 10 lower compared to the STEREO results. Note that the shown C3 images are not the difference mass images used for the mass determination, therefore the planet Mercury can be seen.

Table 8.1: GCS parameters, CME velocity and determined mass of sample event \#11.

\begin{tabular}{|c|c|c|c|c|c|}
\hline $\begin{array}{c}\text { longitude } \phi \\
-88^{\circ}\end{array}$ & $\begin{array}{c}\text { latitude } \theta \\
8^{\circ}\end{array}$ & $\begin{array}{l}\text { asp. ratio } \kappa \\
0.67\end{array}$ & $\begin{array}{l}\text { half angle } \alpha \\
\quad 38.3^{\circ}\end{array}$ & $\begin{array}{l}\text { tilt } \gamma \\
21^{\circ}\end{array}$ & $\begin{array}{c}v_{\text {apex }} \\
1478 \mathrm{~km} / \mathrm{s}\end{array}$ \\
\hline \multicolumn{2}{|c|}{$\begin{array}{c}\text { COR2 A mass [g] } \\
1 \times 10^{17}\end{array}$} & \multicolumn{2}{|c|}{$\begin{array}{c}\text { COR2 B mass }[\mathrm{g}] \\
9.5 \times 10^{16}\end{array}$} & \multicolumn{2}{|c|}{$\begin{array}{l}\text { C3 mass }[\mathrm{g}] \\
8.8 \times 10^{15}\end{array}$} \\
\hline
\end{tabular}



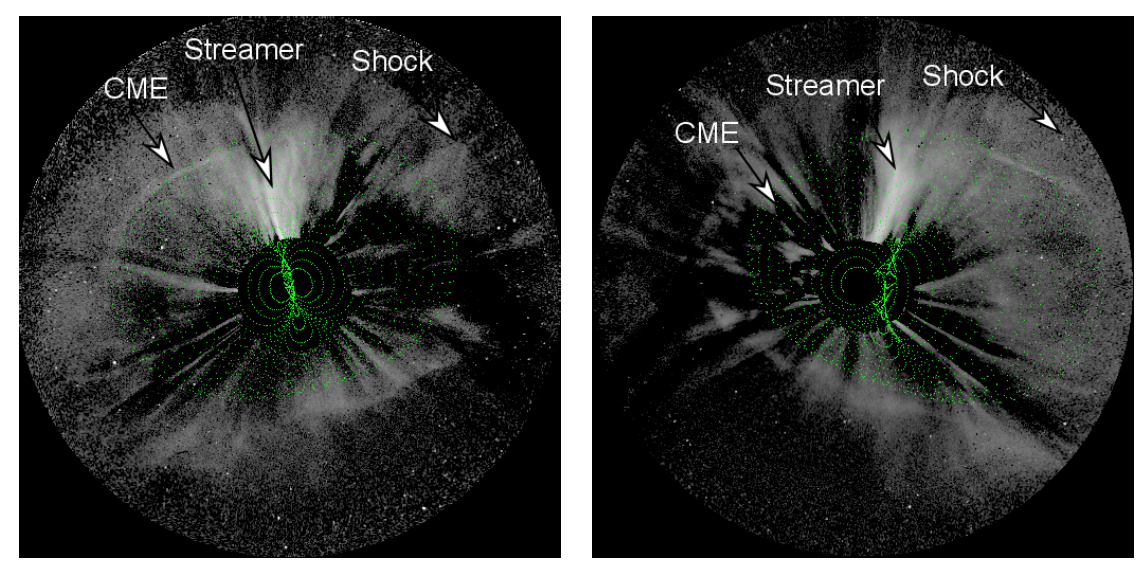

Fig. 8.1: GCS fit of the CME event on 01-10-2011 at 23:54 UT applied to the coronagraph images of STEREO COR2 B(left) and COR2 A(right).
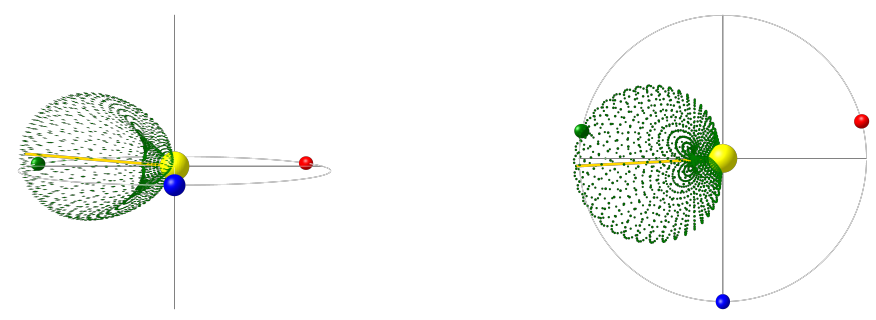

Fig. 8.2: Expansion of 3D GCS grid to an apex height of $1 \mathrm{AU}$ in the HEE coordinate system together with the positions of Earth (blue), STEREO A (red) and STEREO B (green).
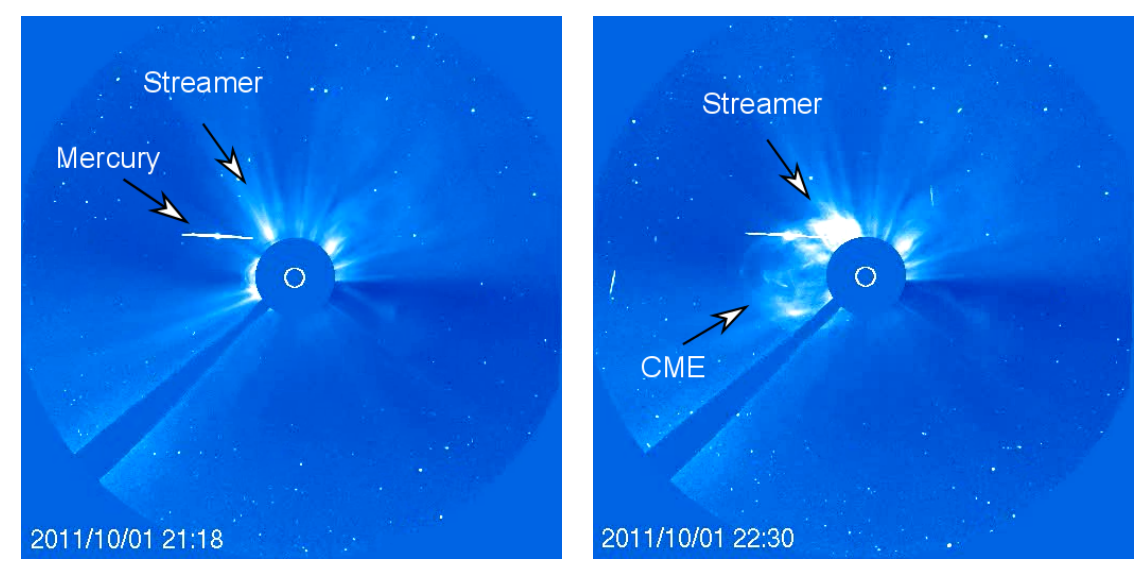

Fig. 8.3: CME observed with SOHO/LASCO C3 on 29-05-2011 at 05:42 and 14:42 [UT]. 
Sample \#12 - Helcats Event \#92

Sample event \#12 occurred on 01-10-2011. To this date both STEREO spacecraft have a separation of $118^{\circ}$. The GCS fit of this event is presented in Fig. 8.4 and the fitting parameters in Table 8.2. The orientation of the GCS grid in relation to earth is presented in Fig. 8.5 and the SOHO C3 observations in Fig. 8.6. The CME is directed towards STEREO B, even if it appears as the wireframe is directed away from it in the COR B image, which is an optical illusion. The mass determination of this event yields masses above $10^{17} \mathrm{~g}$ in both STEREO satellites. Obviously, both detected mass in COR 2 surpasses the event masses detected in LASCO, because the streamer in LASCO could be excluded from the ROI. Interestingly, the detected COR 2A mass also surpasses the mass in COR 2B significantly. This is a consequence of the scaling of the CME mass images with the angular propagation distance away from the POS in each image. In this event the streamer is almost perpendicular to the propagation of the CME, so that this scaling result in an apparent mass asymmetry between both STEREO coronagraph. In this case the CME mass plus streamer mass should be averaged from both detected values.

Table 8.2: GCS parameters, CME velocity and determined mass of sample event \#12.

\begin{tabular}{cccccc}
\hline $\begin{array}{c}\text { longitude } \phi \\
-114^{\circ}\end{array}$ & latitude $\theta$ & asp. ratio $\kappa$ & half angle $\alpha$ & tilt $\gamma$ & $v_{\text {apex }}$ \\
$8^{\circ}$ & 0.66 & $31^{\circ}$ & $-24^{\circ}$ & $1200 \mathrm{~km} / \mathrm{s}$ \\
\hline COR2 A mass $[\mathrm{g}]$ & COR2 B mass [g] & C3 mass [g] \\
$5.0 \times 10^{17}$ & \multicolumn{2}{c}{$1.2 \times 10^{17}$} & \multicolumn{2}{c}{$2.1 \times 10^{16}$} \\
\hline
\end{tabular}



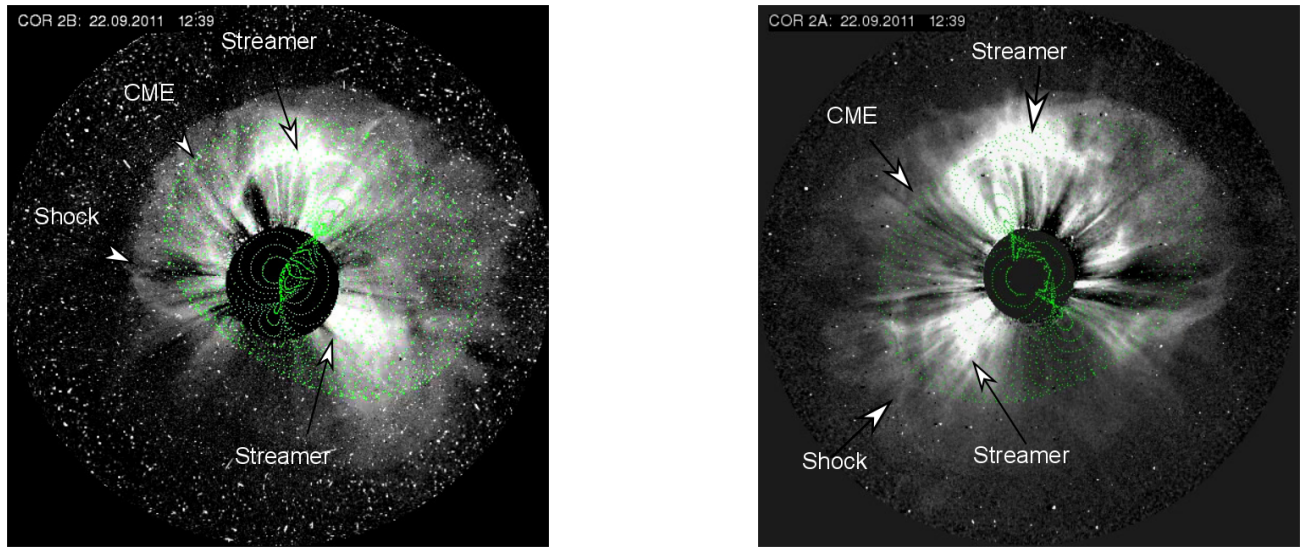

Fig. 8.4: GCS fit of the CME event on 22-09-2011 at 12:39 UT applied to the coronagraph images of COR2 B(left) and STEREO COR2 A(right).
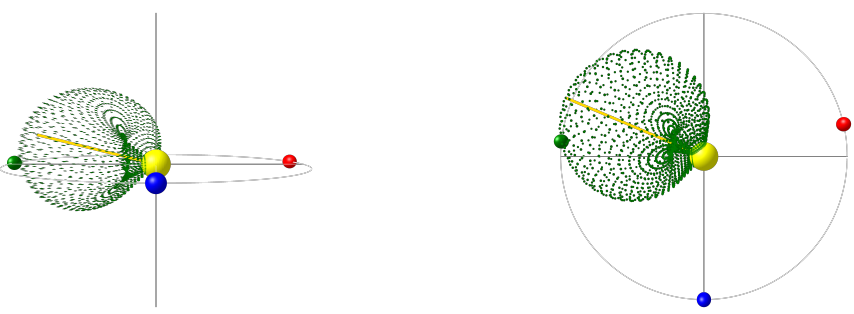

Fig. 8.5: Expansion of 3D GCS grid to an apex height of $1 \mathrm{AU}$ in the HEE coordinate system together with the positions of Earth (blue), STEREO A (red) and STEREO B (green).
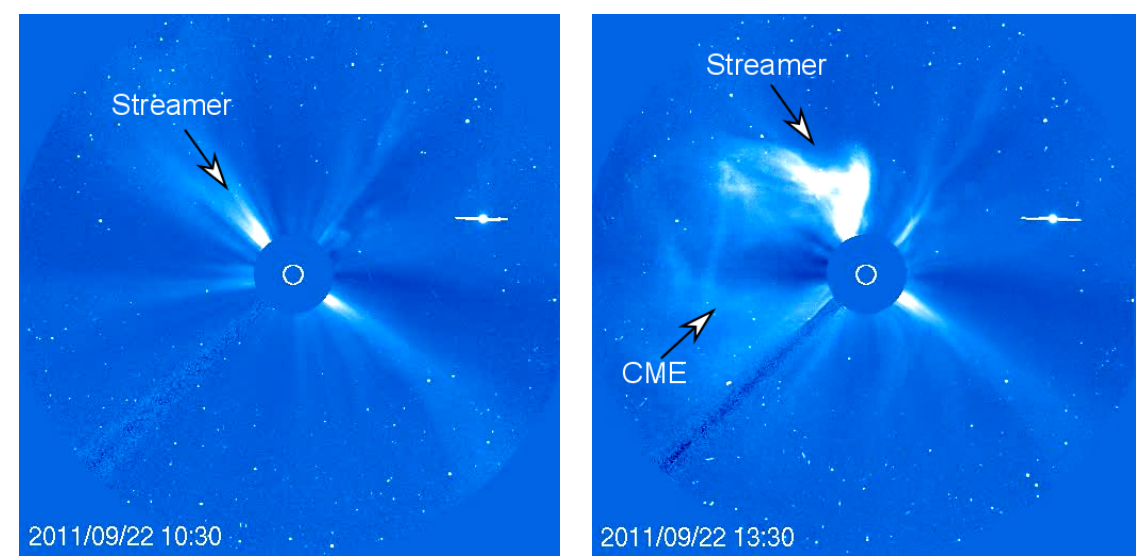

Fig. 8.6: CME observed with SOHO/LASCO C3 on 29-05-2011 at 05:42 and 14:42 [UT].

From this two illustrative examples it appears that the ROIs of disk events cover a larger area of the FOV compared to limb events. Therefore, in such cases, coronal streamers a more likely present within these areas and can contribute significantly to the overestimation of the CME mass. It has to be mentioned, that the mass determination results of LASCO are made with the "plane-of-sky" approach generally tends to underestimate CME masses by up to a factor of 2 (compare with Chapter 8.2). 


\subsection{Mass Loss of the Sun}

If stars are not accrediting gas from the interstellar mediums, they are generally losing mass. Stellar mass loss has a significant impact on stellar and planetary evolution, but also affects the larger-scale evolution of gas and dust in galaxies. Stars, as our Sun, fuse hydrogen into helium. Some of the hydrogen's mass is transformed into energy, which radiates away in the form of photons and neutrinos. The Stefan-Boltzmann law can be used to calculate to solar radiation power:

$$
P_{\odot}=4 \pi R_{\odot}^{2} \sigma T_{\odot}^{4}
$$

with the Stefan-Boltzmann constant $\sigma$, the effective temperature of the Sun $T_{\odot}$ and the radius of the Sun $R_{\odot}$. Einstein's mass-energy equivalence equation yields that the radiation energy of the Sun equals a mass loss of about 4 million tonnes each second. Second, the mass loss of the solar wind is also significant and corresponds to about 1.5 million tonnes per second. This mass loss sums up to about 174 trillion tonnes per year. By assuming a constant mass loss, the Sun only losses about $0.034 \%$ mass in the next 5 billion years. The lower gravitational pull of the Sun has only a small effect on the orbit of Earth. In 65,000 years Earth only moves about one kilometer away from the Sun. At the end of its lifetime, the Sun turns into a red giant and these solar winds are assumed to become much stronger. Even the current effects of the solar wind and CME mass loss rate are negligible, the Sun has also changed considerably over its lifetime, thus prediction models for both are be very helpful to model stellar rotational evolution or particle ablation of planetary atmospheres. From the observation of solar cycles 21 and 22, Wang (1998) have derived an empirical correlation between the solar wind mass loss rate and the sunspot number:

$$
\dot{M}_{\text {wind }} \approx 3.5^{-17}(S S N+570) M_{\odot} \mathrm{yr}^{-1}
$$

where SSN is the recently revised month-averaged sunspot number from the World Data Center (WDC) Sunspot Index and Long-term Solar Observations (SILSO) program. The mass loss decrease of about roughly $50 \%$ at solar minimum can be explained by the relative slower density increases of slow solar wind compared to the relative decreases of wind speed. This solar wind mass loss rate can be compared to the CME mass loss rate. For a subset of 6379 CMEs between 1996 March and 2013 June, R. Cranmer (2017) correlated the mass loss rate due to CMEs to the monthly sunspot number:

$$
\dot{M}_{\mathrm{CDAW}} \approx 5.6 \times 10^{-18}(S S N+7) M_{\odot} \mathrm{yr}^{-1}
$$

The values have been multiplied by a constant factor of 1.5 to account for likely mass underestimations with the former methods and detection limits. It was assumed that these values are still underestimated. The CDAW data indicate that CME mass loss rate at solar maximum contributes only about $3 \%$ to the mass loss rate of the solar wind. Earlier studies by Howard et al. (1985); Webb \& Howard (1994) estimated about 15\%. In this thesis the intense KinCat events are used to calculate an upper limit of the mass loss rate. In Figure 8.7 the average annual CME masses of the catalogue are correlated with the annual average of the monthly sunspot number. The linear fitting yields:

$$
\log _{10}\left(M_{\text {KinCat }}\right) \approx 15.51( \pm 0.02)+0.0032( \pm 0.0004) S S N .
$$

By the investigation of solar cycle 23 Shrivastava \& Jaiswal (2009) derived the following relation between the daily occurrence rate $\mathrm{R}$ of CMEs and the monthly sunspot number:

$$
R_{\text {occur }}=\frac{S S N+0.67}{23.43} .
$$




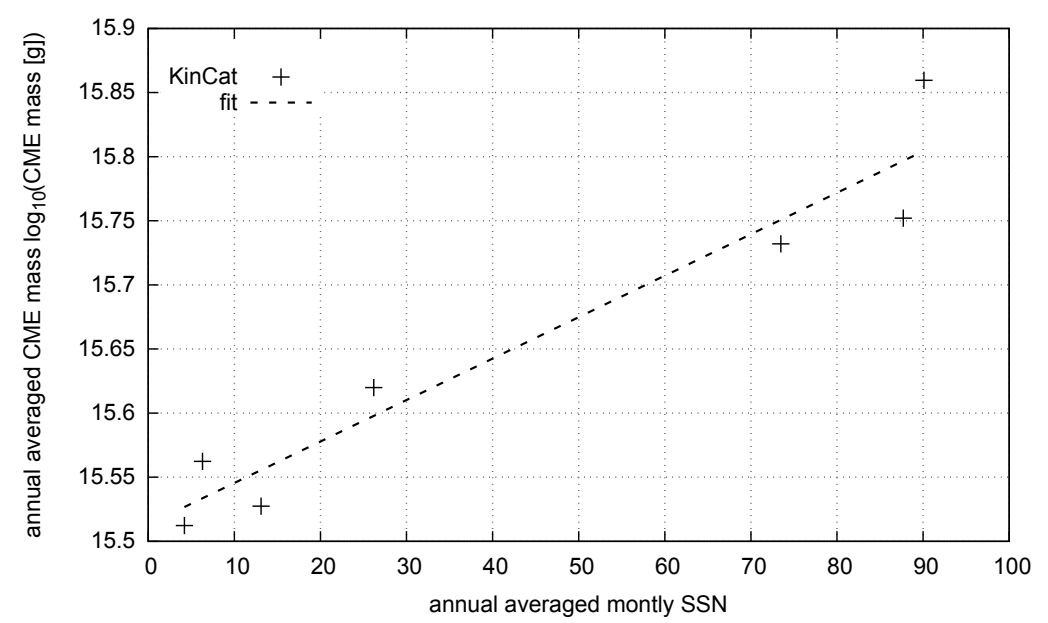

Fig. 8.7: Correlation of the annual averaged CME mass of the KinCat for 122 events between 2007 and 2013 and the annual averaged monthly sunspot number SSN, which are provided by the World Data Center (WDC) Sunspot Index and Long-term Solar Observations (SILSO) program.

From Equation 8.4 and 8.5 the current upper limit of the mass loss rate $\dot{M}_{\text {KinCat }}$ was calculated by the revised month-averaged sunspot number provided by the World Data Center (WDC) Sunspot Index and Long-term Solar Observations (SILSO) program. In Figure 8.8 this upper limit is compared to the $\dot{M}_{\mathrm{CDAW}}$ and $\dot{M}_{\text {wind }}$.

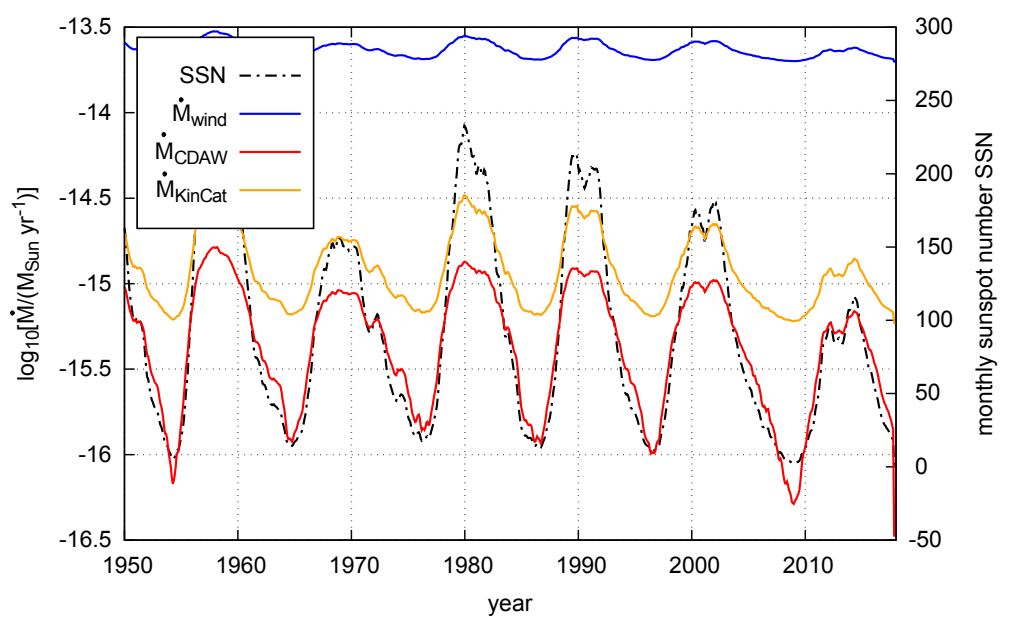

Fig. 8.8: Comparison of the estimated mass loss rates due to the solar wind and for CMEs. The $\dot{M}_{\text {wind }}$ is derived from observations of the solar cycles 21 and 22 by Wang (1998). The mass loss rate $\dot{M}_{\text {CDAW }}$ is based on the CDAW catalogue and derived by R. Cranmer (2017) from a subset of 6379 CMEs between 1996 and 2013. The mass loss rate $\dot{M}_{\text {KinCat }}$ is based on the KinCat catalogue and derived from a set of 122 events between 2007 and 2013.

The upper limit correlation suggests that the CME masses can not contribute more than $10 \%$ to the solar wind mass loss rate. However, analytic modelling of stellar wind mass-loss rates by R. Cranmer (2017); Cranmer \& Saar (2011) indicates that CMEs from the "young Sun" carried 10 to 100 times more mass than the solar wind. Therefore, the cumulative CME mass lost by the young Sun may have been in the order of $1 \%$ of a solar mass. 


\section{Application in CME Forecasts}

This chapter demonstrates the application of the modelling methods and statistical results from the previous chapters to a kinematic CME model to predict terrestrial CME arrivals. The advantage of this model is, that it relies only on coronagraph observations and empirical correlations, making it applicable in real-time space weather forecasts. First, the kinematic model termed Drag Based Model is presented and its application to dense and fast CMEs is discussed. Thereafter, the geometrical GCS model is embedded into the HEE coordinate system to derive the initial CME speed in the direction of Earth. On the basis of geometrical CME modelling results and CME mass determination, the arrival of CMEs can be predicted. Instead of CME mass determinations, the required mass values can also be obtained from the empirical relation between CME mass and velocity as presented in the last chapter.

\subsection{Drag Based Model}

A recent model to determine the CME propagation throughout the heliosphere is the Drag Based Model (DBM) (Vršnak et al., 2013). After the Lorentz force driven acceleration phase, at some point the magnetohydrodynamic drag becomes a dominant factor in the CME dynamics. The drag is a consequence of collisionless transfer of momentum and energy between the CME and the ambient solar wind by MHD waves. The CME kinematics are described through the summation of the acting forces including a viscous drag force

$$
\dot{\boldsymbol{v}}=\frac{\boldsymbol{F}_{L}-\boldsymbol{F}_{\mathrm{grav}}-\boldsymbol{F}_{\mathrm{drag}}}{m}
$$

where $\boldsymbol{F}_{L}$ is the Lorentz force, $\boldsymbol{F}_{\text {grav }}$ the gravity, $\boldsymbol{F}_{\text {drag }}$ the drag force and $m$ the mass of the considered rope segment (Vršnak \& Žic, 2007; Vršnak et al., 2010). In the very low corona up to some solar radii, the Lorentz force dominates which leads to a CME acceleration. The drag force per mass unit $f_{\text {drag }}$ can be written as

$$
f_{\text {drag }}=\gamma(v-w)|v-w|
$$

with the ambient solar wind speed $w$, the CME speed $v$ in an arbitrary direction and the drag parameter $\gamma$. The quadratic formulation of the drag force is consistent with the statement, that interplanetary CMEs that are faster than the ambient solar are decelerated, whereas slower ones are accelerated (Gopalswamy et al., 2000). The differential form of equation (9.2)

$$
\frac{\mathrm{d}^{2} r}{\mathrm{~d} t^{2}}=-\gamma(r)\left(\frac{\mathrm{d} r}{\mathrm{~d} t}-w(r)\right)\left|\frac{\mathrm{d} r}{\mathrm{~d} t}-w(r)\right|
$$

leads to the analytic formulation of the CME velocity if $w(r)$ is assumed to be constant. Its solution can be written as

$$
v(t)=\frac{v_{0}-w}{1 \pm \gamma\left(v_{0}-w\right) t}+w
$$


or as

$$
r(t)= \pm \frac{1}{\gamma} \ln \left[1 \pm \gamma\left(v_{0}-w\right) t\right]+w t+r_{0}
$$

with

$$
\gamma=c_{d} \frac{A \varrho_{w}}{M+M_{v}}
$$

as well as the dimensionless drag coefficient $c_{d}$, the CME cross-section $A$, the ambient solar wind density $\varrho_{w}$, the CME mass $M=\varrho V$ and the virtual CME mass $M_{v} \sim \varrho_{w} V / 2$.

Numerical MHD simulations have further shown that the drag coefficient $c_{d}$ converges to 1 for CMEs (and 0.5-0.8 for shocks waves) shortly after $12 \mathrm{R}_{\odot}$ (Cargill, 2004). For dense CME where $\varrho_{\mathrm{CME}} \gg \varrho_{\text {wind }}$ the virtual mass becomes negligible whereas the CME mass becomes constant above a few solar radii (Bein et al., 2013) and Equation 9.6 can be rewritten as:

$$
\gamma=\frac{c_{d} A \varrho_{w}}{M}
$$

It is further common to substitute $\gamma$ with

$$
\Gamma=\gamma / 10^{-7} \mathrm{~km}^{-1}
$$

A distance dependant solar wind speed model can be found in Sheeley et al. (1997, 1999b), but in this work we use the same approach as Žic et al. (2015) and consider the solar wind speed as constant - at least above $12 \mathrm{R}_{\odot}$.

The solar wind density is calculated by the proton mass $m_{p}$ and the particle density $n_{0}$ :

$$
\varrho_{w}(r)=m_{p} n_{0}(r)
$$

For the particle density the first order approximation of the empirical solar wind model in Leblanc et al. (1998) is used:

$$
n_{0}(r) \simeq 3.3 \times 10^{5} \mathrm{~cm}^{-3} / R^{2} .
$$

From the two common approximations $A \sim R^{2}$ and $\rho_{\text {wind }} \sim 1 / R^{2}$ one can derive:

$$
A \varrho_{\text {wind }}=\text { const. }
$$

As consequence, $\Gamma$ can be indeed regarded as constant above $12 \mathrm{R}_{\odot}$.

Statistically derived $\Gamma$ values for magnetic ejecta have a range of $0.1-2$ (Vršnak et al., 2013), but Venzmer \& Bothmer (2017) displayed that the solar wind density commonly scatters about half a magnitude from their used solar wind particle density of $10^{3} \mathrm{~cm}^{3}$. Therefore, the valid range should be at least expanded to $\Gamma=0.02-2$. Such low $\Gamma$ are common in drag modelling results (e.g. Temmer \& Nitta 2015a.) In Figure 9.1 the CME kinematic was simulated with the DBM for constant $\Gamma$ values and a constant solar wind speed. The modelled initial speed range represents the observed speed range of CMEs in the inner heliosphere which lies between 300 and 3000 km/s (Gopalswamy, 2004; Yashiro et al., 2004). The results show that for small $\Gamma$ values uncertainties in the initial speed result in almost equally large uncertainty of the arrival speed at $1 \mathrm{AU} \approx 215 \mathrm{R}_{\odot}$. In Figure 9.2 CME arrival times at $1 \mathrm{AU}$ are simulated with the DBM for different initial velocities for a constant $\Gamma$ and different constant solar wind speeds. One can notice that $\Gamma=0.04$ the predictions are quasi independent of the solar wind speed. It is still controversially 

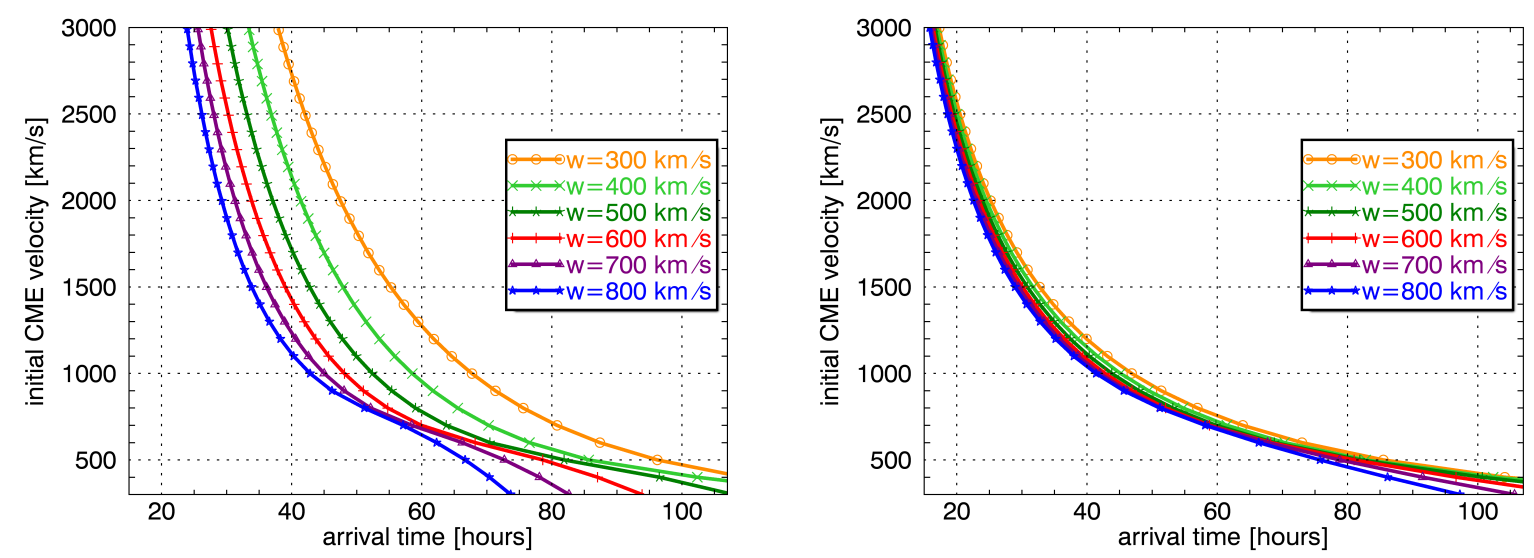

Fig. 9.1: DBM velocity evolution after Vršnak et al. (2010) from $1 \mathrm{R}_{\odot}$ to $1 \mathrm{AU}$. The CME speeds are simulated for a constant solar wind speed of $500 \mathrm{~km} / \mathrm{s}$ and a constant $\Gamma=0.2$ (left) and 0.04 (right).
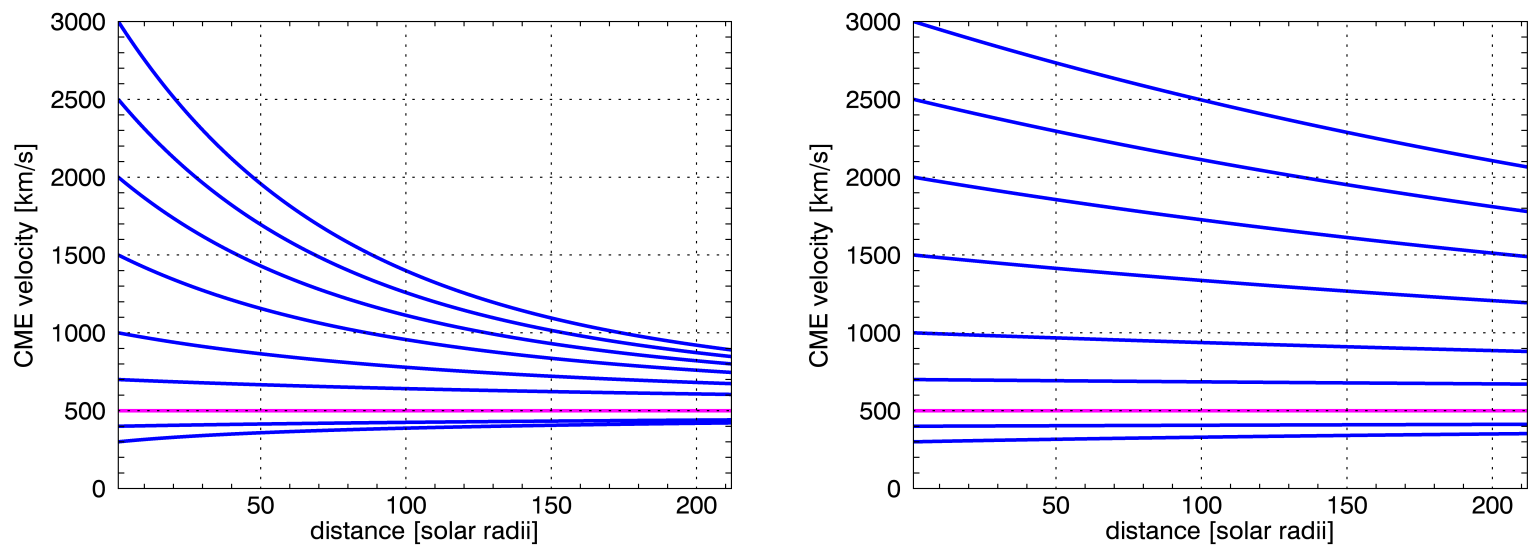

Fig. 9.2: DBM travel time simulation after Vršnak et al. (2010) from $1 R_{\odot}$ to $1 \mathrm{AU}$. The arrival time at $1 \mathrm{AU}$ is simulated with different initial velocities at $1 \mathrm{R}_{\odot}$ for a constant $\Gamma=0.2$ (left) and 0.04 (right) and for various constant solar wind speeds $v_{\text {wind }}$. 
discussed at which point the drag force becomes dominant, so that the DBM model can be applied. Obviously the model fails if it is initiated at heights where the CME is still driven by the Lorenz force (Gallagher et al., 2003). It was suggested by Carley et al. (2012) and Byrne et al. (2010) that the drag force is dominating in a very early phase of CME propagation above $7 \mathrm{R}_{\odot}$. For a statistic analyses of over 16000 CMEs within the FOV of LASCO the velocity dependant manifestation of the three phases of propagation (Lorentz dominated, equilibrium of forces and drag dominated) was described by Michalek et al. (2015). They concluded that the outer edge of the LASCO FOV is drag dominated. In a recent work of Sachdeva et al. (2017) it is shown that the Lorentz force becomes negligible as early as $3.5-5$ solar radii for fast events and only at $12-50 \mathrm{R}_{\odot}$ for slow events.

\subsection{The DBM plus GCS Model}

The speed-distribution of the CME front is not homogeneous, but has its peak at the apex and drops towards the flanks. Therefore it is crucial to know which component of the CME front is directed towards Earth. In Figure 9.3 an illustrative example is presented, where the apex of the CME front reaches Earth at $1 \mathrm{AU}$ about 4 hours before the flanks reach the same distance. In terms of CME forecast one is more interested in the initial speed
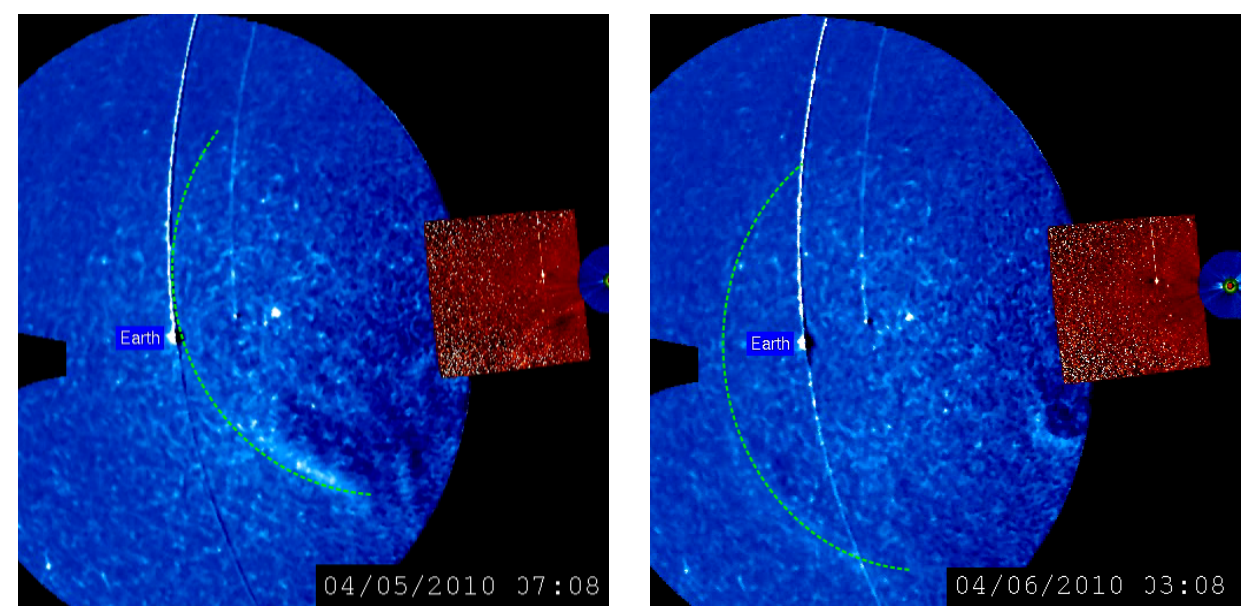

Fig. 9.3: Combined view of the SECCHI B instruments HI-2, HI-1, COR2, COR1 and EUVI on early April 2010. The front of a CME can be observed for an event. The CME front is highlighted with dashed lines. (Adapted from SECCHI combined movies) ${ }^{1}$

in Earth-direction than in apex-direction. This speed can be derived with the GCS model from the relation of the shape height in the direction of the CME apex $h_{\text {apex }}$ and in the direction towards Earth $h_{\text {earth }}$. By assuming self-similar expansion (e.g Chen 1996; Chen et al. 2006; Cremades \& Bothmer 2004) around $12 \mathrm{R}_{\odot}$, we can derive the CME speed from the forward modelling results of the apex speed and the GCS shape

$$
v_{\text {earth }}=h_{\text {earth }} / h_{\text {apex }} \cdot v_{\text {apex }} .
$$

It is not trivial to derive the term $h_{\text {earth }} / h_{\text {apex }}$ analytically for any GCS shape, but it can easily be derived numerically, as executed in this work. Also, the bias between the apex speed and the Earth-directed speed is most significant if only the CME flank is directed towards Earth.

\footnotetext{
${ }^{1}$ http://secchi.nrl.navy.mil
} 
For the application of the GCS model to the DBM model, the cross-section must be derived from the GCS model parameters. Instead of using the given approximation in Temmer \& Nitta (2015b), the GCS cross-section calculated by the approximation with the base area of elliptic cone at the flux rope centre height $R_{c}$ with the two half angles of the two main axis $\alpha$ and $\delta$ :

$$
A=\tan \alpha \tan \delta\left(R_{c}\right)^{2} \pi,
$$

where $\delta=\arcsin \kappa$ and $R_{c}=R /(1+\kappa)$.

\subsection{Prediction Example}

The application of GCS modelling and mass determination to CME forecasts is presented by an illustrative example. It is shown how the CME parameterisation can be combined with the DBM model to predict the terrestrial arrival of the July 12, 2012 CME. In Figure 9.4 the COR2 GCS modelling of this event is shown at $12 \mathrm{R}_{\odot}$ around $18 \mathrm{UT}$. The fitted GCS parameters are presented in Table 9.1. The heliospheric orientation is presented together with the satellite configuration in Figure 9.5.

\section{Helcats Event \#111 - Date 12-07-2012}
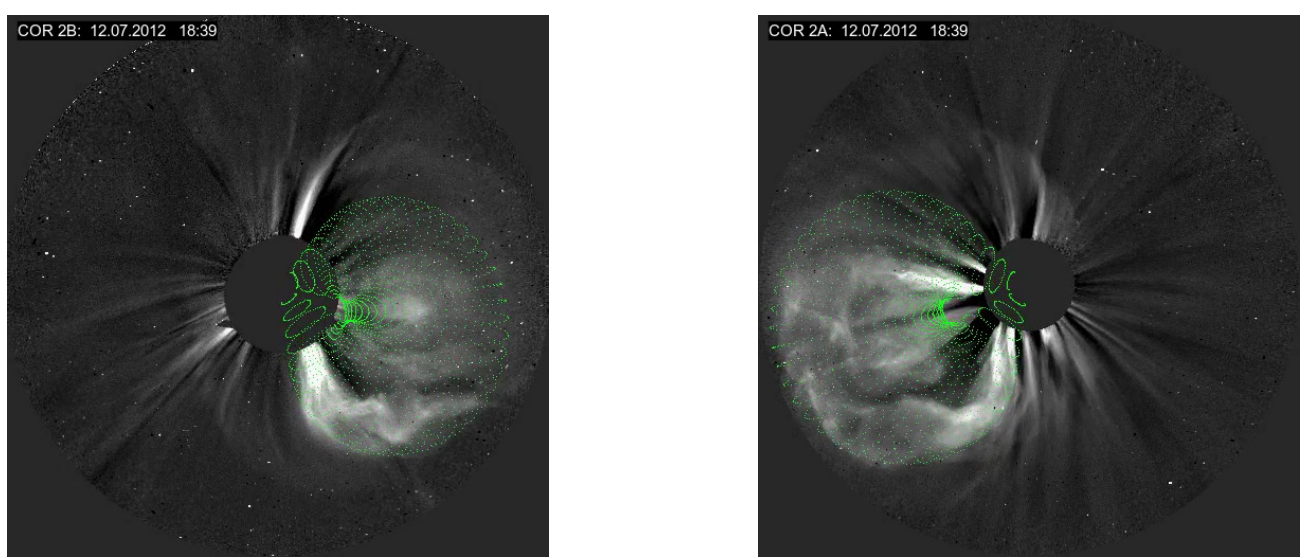

Fig. 9.4: GCS fit of the CME event on 12-07-2012 at 18:39 UT applied to the coronagraph images of COR2 B(left) and STEREO COR2 A(right).
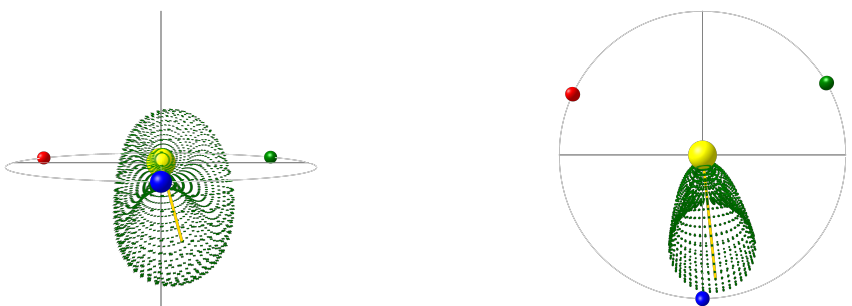

Fig. 9.5: Expansion of 3D GCS grid to an apex height of $1 \mathrm{AU}$ in the HEE coordinate system together with the positions of Earth (blue), STEREO A (red) and STEREO B (green). 
Table 9.1: GCS parameters, CME velocity and determined mass in coronagraph.

\begin{tabular}{cccccc}
\hline $\begin{array}{c}\text { longitude } \phi \\
8^{\circ}\end{array}$ & latitude $\theta$ & asp. ratio $\kappa$ & half angle $\alpha$ & tilt $\gamma$ & $v_{\text {apex }}$ \\
& $-12^{\circ}$ & 0.46 & $31^{\circ}$ & $90^{\circ}$ & $1239 \mathrm{~km} / \mathrm{s}$ \\
\hline COR2 A mass $[\mathrm{g}]$ & \multicolumn{2}{c}{ COR2 B mass [g] } & \multicolumn{2}{c}{ C3 mass $[\mathrm{g}]$} \\
$1.6 \times 10^{16}$ & \multicolumn{2}{c}{$1.3 \times 10^{16}$} & \multicolumn{2}{c}{$6.9 \times 10^{15}$} \\
\hline
\end{tabular}

The event is clearly Earth-directed, has an initial apex speed of $1239 \mathrm{~km} / \mathrm{s}$ and a mass of $1.5 \times 10^{16} \mathrm{~g}$. For the same event, Hess \& Zhang (2014) derived an apex speed of $1423 \mathrm{~km} / \mathrm{s}$ at $5 \mathrm{R}_{\odot}$ from stereoscopic GCS fitting, which is reasonable. Besides, in their modelling the southern brightness enhancement is not considered as part of the CME, which is not supported by this author. In the CDAW catalogue, the event appears as halo event in the LASCO C2 with a linear projected speed of $884 \mathrm{~km} / \mathrm{s}$ below $4 \mathrm{R}_{\odot}$. The derived CME mass of $6.9 \times 10^{15} \mathrm{~g}$ which is very likely underestimated for measurements at such low heights. The CME was detected at L1 by ACE about 59 hours later at a speed of $590 \mathrm{~km} / \mathrm{s}$, which is discussed in the next section in more detail.

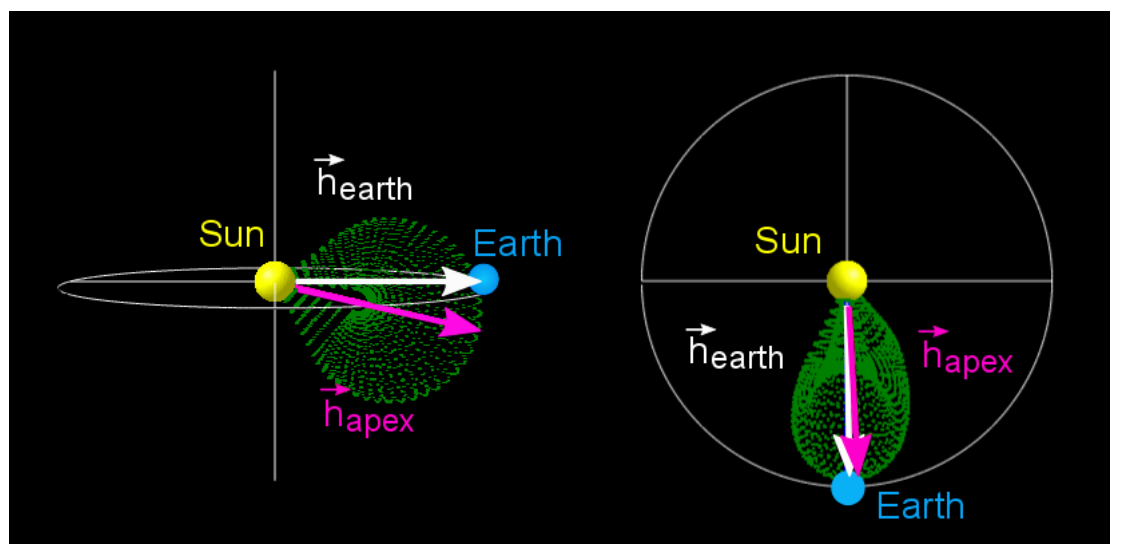

Fig. 9.6: GCS-shape in the HEE coordinate system of the CME event on July 12, 2012. For visualisation purposes, the shape was expanded to $1 \mathrm{AU}$. Further, the height vector of GCS shape in the main axis and in Earth-direction are shown.

The relation between the height of the GCS shape towards Earth $h_{\text {earth }}$ and the apex height $h_{\text {apex }}$ in Figure 9.6 is determined numerically at $12 \mathrm{R}_{\odot}$ and yields:

$$
h_{\text {earth }} / h_{\text {apex }}=0.95 \text {. }
$$

Equation 9.12 is used to calculate the initial speed towards Earth :

$$
v_{\text {earth }}=1172 \mathrm{~km} / \mathrm{s} .
$$

Further, the GCS cross-section is calculated with Equation 9.13:

$$
A=3.2 \times 10^{19} \mathrm{~m}^{2} .
$$

Equation 9.7 is now used to together with Equation 9.8 to calculate $\Gamma$ for different solar wind densities and CME masses. The solar wind density itself $\varrho_{w}=n m_{p}$ is determined from an estimated number density and the proton mass $m_{p}=1.672 \times 10^{-27} \mathrm{~kg}$.

The solar wind parameters and CME masses of the two first simulations are chosen to calculate an upper and lower limit of the CME arrival. The mass result of this work and a mass comparable to the mass measurement of the CDAW catalogue are used as limits. 
Table 9.2: CME arrival prediction parameters.

\begin{tabular}{c|cccc|ccc}
\hline simulation & \multicolumn{4}{|c|}{ input } & \multicolumn{3}{c}{ result } \\
& $v_{\text {wind }}$ & $n_{\text {wind }}$ & $v_{\text {CME }}$ & $M_{\text {CME }}$ & $\Gamma$ & $T_{\text {arr }}$ & $v_{\text {arr }}$ \\
\hline upper limit & $800 \mathrm{~km} / \mathrm{s}$ & $2 \times 10^{9} \mathrm{~m}^{-3}$ & $1172 \mathrm{~km} / \mathrm{s}$ & $1.5 \times 10^{16} \mathrm{~g}$ & 0.07 & $34 \mathrm{~h}$ & $1081 \mathrm{~km} / \mathrm{s}$ \\
lower limit & $300 \mathrm{~km} / \mathrm{s}$ & $8 \times 10^{9} \mathrm{~m}^{-3}$ & $1172 \mathrm{~km} / \mathrm{s}$ & $0.5 \times 10^{16} \mathrm{~g}$ & 0.86 & $94 \mathrm{~h}$ & $333 \mathrm{~km} / \mathrm{s}$ \\
best estimate & $350 \mathrm{~km} / \mathrm{s}$ & $3 \times 10^{9} \mathrm{~m}^{-3}$ & $1172 \mathrm{~km} / \mathrm{s}$ & $1.0 \times 10^{16} \mathrm{~g}$ & 0.16 & $50 \mathrm{~h}$ & $600 \mathrm{~km} / \mathrm{s}$
\end{tabular}

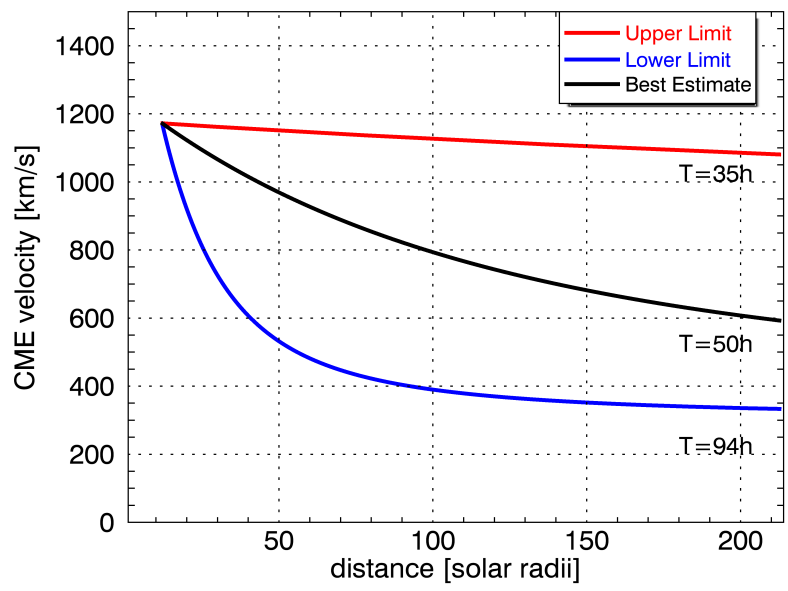

Fig. 9.7: Simulation of the terrestrial CME arrival time and speed of the July 12, 2012 event. The CME arrivals are simulated based on the CME parameters in Table 9.2.

From the in-situ data we have gathered the following arrival times and speeds:

$$
\begin{aligned}
T_{\text {in-situ }} & =59 \mathrm{~h}, \\
v_{\text {in-situ }} & =590 \mathrm{~km} / \mathrm{s} .
\end{aligned}
$$

It becomes obvious that upper and lower limits of solar wind conditions and the CME masses cause a huge spread in the predictions:

$$
\begin{aligned}
\Delta T_{\text {limits }} & =59 \mathrm{~h} \\
\Delta v_{\text {limits }} & =748 \mathrm{~km} / \mathrm{s},
\end{aligned}
$$

This huge spread is a main reason for the high uncertainties in CME forecasts as both, the CME mass and the solar wind conditions, are difficult to estimated in advance. The third simulation is a "best estimate". In this case, the in-situ solar wind parameters only a few hours before arrival of the CME shock are used. Further, the input mass value was reduced by about a third of the measured value to account for the fact, that it is not equally distributed among the CME. This is also more consistent with the CME massvelocity correlation from the last chapter. The "best estimate" simulation yields:

$$
\begin{aligned}
T_{\text {best estimate }} & =50 \mathrm{~h}, \\
v_{\text {best estimate }} & =600 \mathrm{~km} / \mathrm{s} .
\end{aligned}
$$

This prediction is very accurate in terms of the arrival speed but the simulated CME arrives 9 hours ahead of the actual empirical in-situ signatures. 
In numerical weather predictions, a concept termed "Ensemble Forecasting" is often used, which can be considered as some form of Monte Carlo analysis. Instead of a single forecast of the most likely whether a set of forecasts is produced, which indicates the possible ranges of the future weather. The most likely future state of a system can than be determined from the ensemble average, which has been applied to CME forecasts (e.g. Mays et al. 2015). The concept shall be demonstrated in a nutshell by either considering the upper and lower limit as ensemble,

$$
\begin{aligned}
& T_{\text {avg-limits }}=62.5 \mathrm{~h}, \\
& v_{\text {avg-limits }}=707 \mathrm{~km} / \mathrm{s},
\end{aligned}
$$

or all three simulations

$$
\begin{aligned}
T_{\text {avg-limits }} & =60 \mathrm{~h}, \\
v_{\text {avg-all }} & =571 \mathrm{~km} / \mathrm{s} .
\end{aligned}
$$

Generally, a larger ensemble should improve the accuracy of the prediction. However, in this case the ensemble prediction from only 3 simulations is extremely accurate, which is likely fortunate.

\subsection{In-Situ Arrival}

Hess \& Zhang (2014) have equally tried to model this event with the DBM, but have associated the CME front to the passing ejecta instead of the arrival of the magnetic flux rope. For that reason their results are not presented. Instead, the connection between this CME and its in-situ signatures is discussed in this section. This interpretation of the in-situ data of the July,12 2012 event was made independently from Shen et al. (2014) but comes to similar results.

Once propagating further out into the heliosphere the term Interplanetary Coronal Mass Ejections (ICMEs) is frequently used to describe the interplanetary manifestation of CMEs in the solar wind. Typical ICME signatures within the solar wind are recapitulated in Zurbuchen \& Richardson (2006). It was stated that the in-situ response of the ICME front are MC cloud signatures. Such in-situ signatures are identified through the measurements of the magnetic field components, temperature, proton density and solar wind velocity. The signatures occurred in the order of a leading shock front, a turbulent sheath region and the ICME plasma driver gas. Depending on the ICME orientation towards earth, not all of them have to be detectable at L1. Figure 9.8 presents the in-situ measurements of 12 th July 2012 event at L1. The arrival of a shock wave manifests in a sudden rise of the in-situ measured proton speed and temperature. The post shock region turbulences are created by the shock sheath provoking the fluctuations of the magnetic field, the so called "field line drapping". Gosling \& McComas (1987). The ICME driver gas becomes manifest in a 'magnetic clouds' (MC), which where described by Klein and Burlaga (1982) as enhanced magnetic fields $(|B| \geq 10 \mathrm{nT})$ with a slow angle rotation as well as low proton temperatures and a low ratio between thermal and magnetic field energies. Bothmer and Schwenn (1996) analysed that for a set of 25 shock disturbance events, $68 \%$ were followed by a clear driver gas signature and $41 \%$ of the driver gases were magnetic clouds. Eight different categorise of MC clouds where defined by Bothmer \& Schwenn (1998) which where in the following extended by the concept of "Magnetic Cloud like" (MCL) structures (Lepping et al., 2006). 


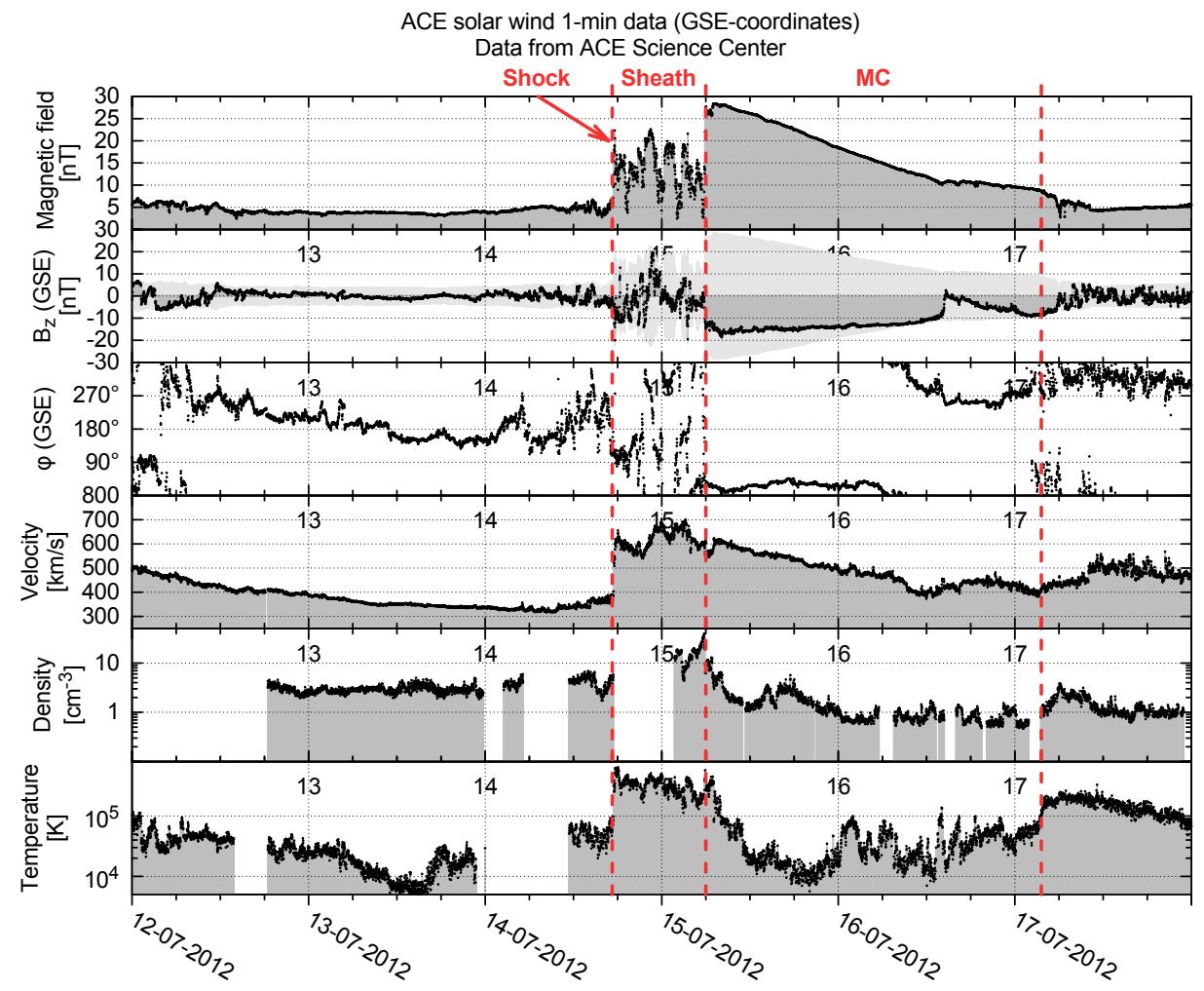

Fig. 9.8: ACE solar wind 64s data (GSE coordinates) for ICME counterpart to the 12th July 2012 CME, downloaded form ACE Science Center. In the common solar wind ( $w=350 \mathrm{~km} / \mathrm{s})$, a clear shock $\left(v_{\text {shock }}=600 \mathrm{~km} / \mathrm{s}\right)$ is followed by a high turbulent magnetic field signature together with a high proton temperatures. This sheath region is so then followed magnetic cloud. The temperature drops over one order, the velocity is slowly but continuously decaying. An abrupt rising and slowly decaying absolute magnetic field is clearly visible but the $B z$ component is nonrotating which could also be an argument to categorise this structure as MCL. (Malte Venzmar and Volker Bothmer) 
These MCLs do not seem to be good representatives of MC or Flux rope models. It was argued that for instance, a missing magnetic field rotation might result from non-central piercing at a magnetic flux rope leg.

\subsection{Conclusion}

This section has given a short outline of the potential use of the geometrical modelling and mass determination results of this thesis to CME arrival time predictions. It was demonstrated that the CME kinematics depend on the initial CME speed and the density ratio between the solar wind and CME. From the results of numerical simulations one can derive that non-dense and slow CMEs are accelerated towards the solar wind speed shortly after they have overcome the Lorentz acceleration phase. In such cases geometrical modelling of the CME front is of minor importance to CME forecasts. On the other hand, extremely dense and fast CMEs almost completely decouple from the drag-deceleration. Here, the CME predictions mostly depend on geometrical modelling results of the CME front. This is especially true if the CME is seen as partial halo from the target, so that the initial speed component has derived from geometrical modelling. However, such extreme cases are very rare so that the influence of the solar wind cannot generally be ignored. This puts CME forecaster in a difficult situation, as they have to also predict the solar wind conditions as well as determine the CME mass in advance, to make CME arrival predictions. In Chapter 8 it was discussed that CME mass determination methods are not accessible in a timely manner. Therefore, it was proposed that the CME mass can be estimated from the CME speed for intense events, based on the derived correlations. Nevertheless, all these assumptions are based on idealised events. A more reliable model also has to take transients in the solar wind into account, as for example CME-CME interaction (Liu et al., 2012; Temmer et al., 2014), which is currently not well understood. However, with the here presented ensemble modelling approach we have reached an accurate CME prediction just from the upper and lower prediction limit. It was shown, that this prediction can be further improved if the solar wind conditions and the CME mass are well estimated. 


\section{Summary}

This thesis has presented a combined method to parameterise the kinematic, mass and morphology of CMEs based on coronagraph observations. The method was developed to compare the different characteristics of CMEs in a consistent and comparable manner. It is based on the combination of geometric CME modelling with the GCS model and the CME white-light determination technique founded on the basis of Thomson scattering theory and its application to the corona. The model was applied to a set of 122 CME events spawned during the solar cycle 24 and stereoscopically observed with the COR2 coronagraphs of the STEREO twin satellites.

\subsection{Conclusion}

All determined kinematic, geometric and mass parameters of the 122 events are gathered in the "KinCat" online catalogue. This is connected to HI-observations, which will enable many followup studies on the CME propagation throughout the heliosphere.

It shall be emphasized that geometric and mass modelling results are based on the visual interpretation of the modeller. Therefore, experience is an important factor, when it comes to this task. Thus, this thesis presents several fitting examples together with critical comments to provide some of this experience to future modellers. From the analysis of the KinCat events, the following results are obtained:

\section{Morphology}

The CME analysis of the GCS modelling showed that the reconstruction of a 3-dimensional CME structure via their 2-dimensional projections is subject to projection effects. These effects are strongest if the stereoscopical resolution is very low, which is the case if both stereoscopic observers are in conjunction or opposition and the event is directed towards or away from one of them. This statement is consistent with the condition, that the CME appears as disk events in all observing coronagraphs. In such a case the projection effects can lead to an overestimation of the CME morphology by up to a factor of two and an underestimation of the CME speed in the same order of magnitude. These projection effects are most significant under a very active background corona in the form of contemptuous streamers, other CMEs as well as under very bright CME shock fronts. Additionally, the low stereoscopic resolution contributes to the overestimation of the CME morphology due to likely misinterpretation of the CME front by the modeller. If a CME appears as limb event in at least one coronagraph, it was shown that the CME morphology can be deprojected with a much higher level of confidence. 
The average morphology of the CMEs within the data-set can be described with the GCS half angle $\alpha$ and the GCS aspect ratio $\kappa$ via:

$$
\alpha=27.6 \kappa+10.8
$$

This correlation can be used to evaluate future GCS modelling results, especially for disk events. The obtained CME morphology of disk events is more affected by projection effects than the CME velocity. It can be empirically estimated from the CME speed via:

$$
\begin{aligned}
& \alpha=1.11 \times 10^{-2} v_{\text {apex }}+15.0 \\
& \kappa=1.7 \times 10^{-4} v_{\text {apex }}+0.30
\end{aligned}
$$

\section{Mass}

The presented directional mass determination approach combines the directional mass determination with GCS modelling and can provide an upper limit of the CME masses. This method can be combined in further studies with the "POS-mass determination" method, which provides a lower limit, to obtain mass ranges for CMEs. It was demonstrated that this approach delivers reasonable results. However, they tend to overestimate the CME mass due to geometric effects with increasing distance of the CME source region from the POS of the observer. The overestimation reaches on average a factor of 3 for an angular distance of $80^{\circ}$. Projection effects are even more relevant in the determination of the CME mass. It was shown that these projection effects can increase the mass results by a factor of 10 and more if coronal streamers are present. CME mass overestimations caused by coronal streamers only occur if the streamers are present within the 2-dimensional projection of the CME shape and if they are influenced by the CME eruption. This influence can manifest in an increased brightness and a deflection of the streamer. In such cases, the difference imaging approach is not able to subtract the streamers from the mass images. It was discussed that the likelihood of streamers being present within a 2-dimensional CME shape projection is higher if the event is seen as disk event. Therefore, the mass determination of disk CMEs should be avoided for a very active background corona. If more than one coronagraph is used, the lowest mass result have the largest confidence, as they are more likely not be affected by contributions from coronal streamers. Further, the upper limit of the mass loss rate of the Sun due to CMEs was determined to less then $10 \%$ of the mass loss rate caused by the solar wind and only $4 \%$ of the mass loss caused by hydrogen fusion. It was discussed that the current mass loss rate caused by CMEs is not relevant for the future of the solar evolution, however it may have had a more important role for the younger Sun.

\section{Kinematics \& Correlations}

It was discussed that the comparability of CME speeds increases if they are measured in a state where they have overcome the acceleration phase by Lorentz forces. It was derived that this can best be achieved in coronagraphs at a CME height of 12 solar radii. Moreover it was shown, how geometric modelling can be used to derive the CME speed in the direction of Earth. The terrestrial CME arrival of a CME with an earth directed initial speed of $1172 \mathrm{~km} / \mathrm{s}$ was simulated with the GCS plus DBM model and demonstrated that demonstrated that CME arrival predictions are strongly affected by the solar wind density and CME mass. In the presented sample event, typical CME mass and solar wind values yield a large spread of the arrival prediction limits $(\Delta T=59 \mathrm{~h}$ and $\Delta v=748 \mathrm{~km} / \mathrm{s})$. Statistical results of the average CME speed, width, and mass have been successfully 
correlated to the solar activity within the observed time-frame of solar cycle 24 . The selection criterion of intensive and clear visibility led to a data-set of CMEs which are on average larger, faster and heavier than comparable results from former studies. This is especially true during the phases of strong solar activity. Former studies have claimed that the solar activity correlates well with the upper limit average of CME parameters. The presented study confirms this claim. It was also discussed that current mass determination methods are not realisable in a timely manner and therefore not available in the forecast of CMEs. Based on the further parameter correlation of the individual events, it is proposed that the following empirical correlation between the CME mass and the CME speed is most suited to estimate the mass CMEs:

$$
\log _{10}(M)=3.4 \times 10^{-4} v_{\text {apex }}+15.479 .
$$

Further studies could confirm the consistency of these results for the following solar cycles and also analyse the benefits to CME arrival time prediction in a statistical manner.

\subsection{Outlook}

This thesis contributes to the parameterisation of CMEs close to the solar surface. Next, a brief outlook and some thoughts on future methods and infrastructure are given, which can be established to further improve CME parameterisation and CME forecast.

\section{Advanced Geometric Model}

It it with great interest that I have read about the Flux Rope in 3D (FRi3D) model by Isavnin (2016). This fully analytic geometric CME model is based on the GCS model but is also capable of reproducing all major deformations i.e. deflection, rotation and front flattening. Personally, I remain ambivalent about such a model. On the one hand, the

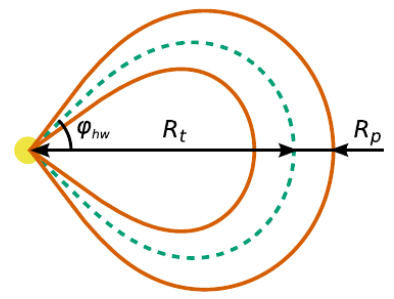

(a)

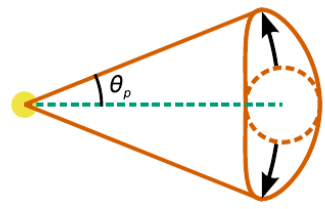

(b)

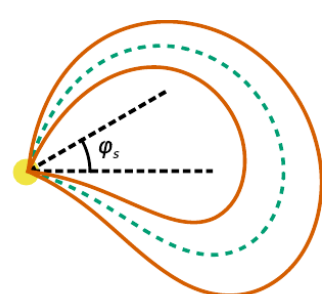

(c)

Fig. 10.1: Schematic of a CME flux rope (a) and its pancaking (b) and skewing (d) deformations which can be reproduced with the FRi3D model. (Isavnin, 2016)

additional modelling parameters or degrees of freedom which such a model provide would be a useful to reproduce the geometric shape of a few CMEs even more accurately. On the other hand, the modelling parameters of the GCS, especially the $\kappa, \alpha$ and $\gamma$ have already two more degrees of freedom than the Cone model. For only small stereoscopic observation angles the GCS already seems to be overdetermined and makes a false claim about how much three-dimensional information are indeed deprojectable from the two-dimensional shape. Therefore, I would prioritise the following point.

\section{Additional Perspectives and L5 Mission}

This thesis has discussed that CMEs must be observed with a sufficient angular separation. Obviously, the STEREO coronagraphs cannot always provide this separation. Many work- 
ing groups have therefore integrated the SOHO LASCO coronagraphs images into our GCS modelling, which provides an additional perspective from Earth. The first GCS modelling results with three coronagraphs of our working group are presented in Figure 10.2.

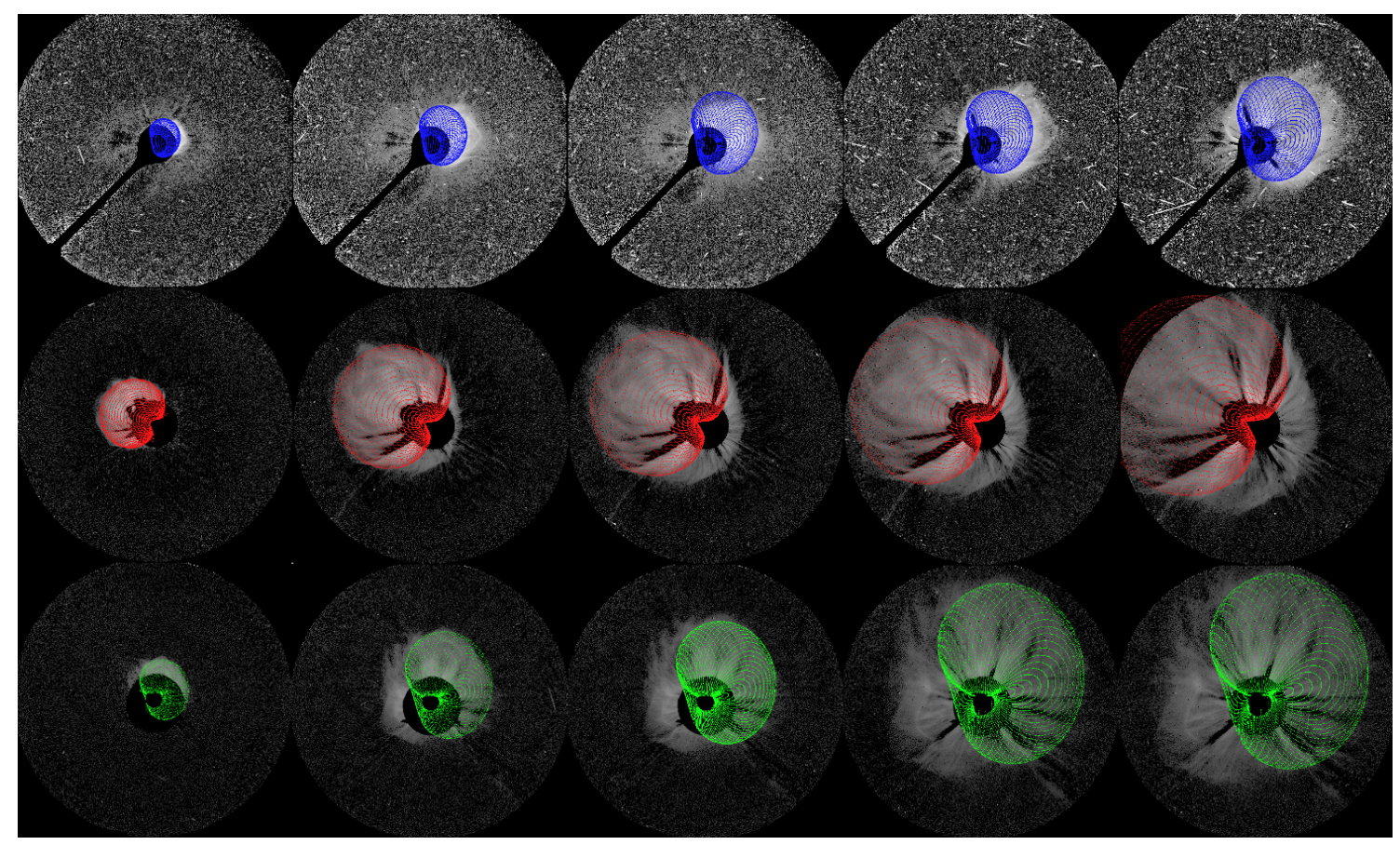

Fig. 10.2: Simultaneous GCS time-series fits of the 13th March 2012 event with SOHO C 3 (blue), STEREO/COR $2 \mathrm{~A}$ (red) and STEREO/COR $2 \mathrm{~B}$ (green). The corresponding STEREO satellite positions are $\phi_{\alpha}=110$ for STEREO A and $\phi_{\beta}=-118$ for STEREO B.

Even with the LASCO coronagraphs positioned at L1 a sufficient stereoscopic observation still depends on the time-specific satellite configurations and the propagation direction of the CME. If all satellites are close to mutual conjunction or opposition then Earth-directed CMEs will still appear as disk events in all coronagraphs. Therefore, this thesis strongly supports the European Space Agency (ESA) mission plans to send a spacecraft to the gravitationally stable point L5 (see Vourlidas 2015, Figure 10.3) by around 2023. The onboard coronagraphs will then provide an continuous angular separation of $60^{\circ}$ to Earth. Compared to the L4, L5 has the further advantage, that potential missions would also be able to observe a preview of the solar surface with its active regions before it rotates towards Earth.

\section{CME Forecast}

Deprojecting the CME morphology with the GCS model can be very valid in terms of CME forecast, but kinematic CME models have to consider the GCS geometry to be beneficial in this context. With the strong support of my working group, I have developed such a tool, which combines the GCS morphology with the DBM propagation model. The concept has been described in Chapter 9. The tool is currently in testing phase and the results look promising. It will hopefully be further maintained and enhanced, for example by a timedependent solar wind model. The global 3D MHD WSA-ENLIL model (Odstrcil, 2003; Odstrcil et al., 2004) provides a such time-dependent description of the background solarwind plasma and magnetic field into which a Cone model of a CME can be inserted. Such an

\footnotetext{
${ }^{1}$ www.metoffice.gov.uk
} 


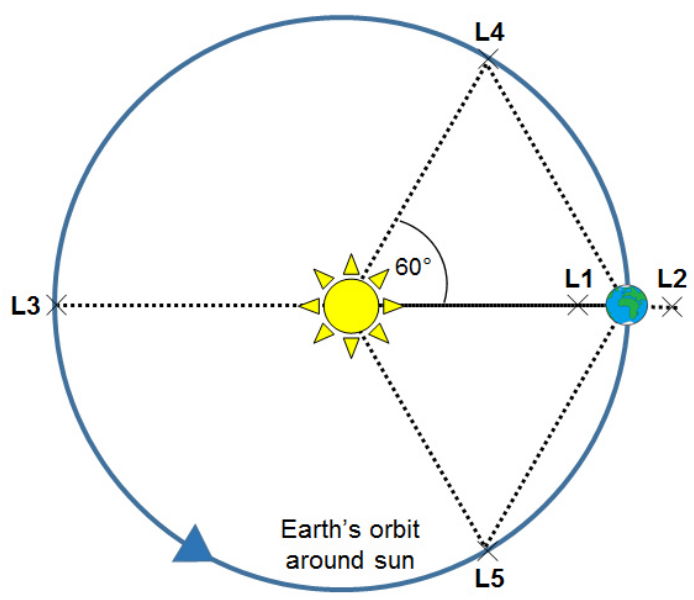

Fig. 10.3: L5 point trailing behind Earth at the same orbit. A spacecraft here would provide a unique perspective on the Sun-Earth system. (Met Office ${ }^{1}$ )

WSA-ENLIL plus Cone model run is presented in Figure 10.4. Also, it would be interesting to implement the GCS model into the WSA-ENLIL model. However, the modelling results of this thesis are already now beneficial for the WSA-ENLIL code as they can be easily adapted to Cone models. Moreover, I would like to support "ensemble predictions" in CME forecasts which I have not exploited in the framework of this thesis to its full extend. The ensemble prediction (Mays et al., 2015) approach provides a probabilistic forecast that includes an estimation of arrival-time uncertainty from the spread in predictions and a forecast confidence in the likelihood of CME arrival.

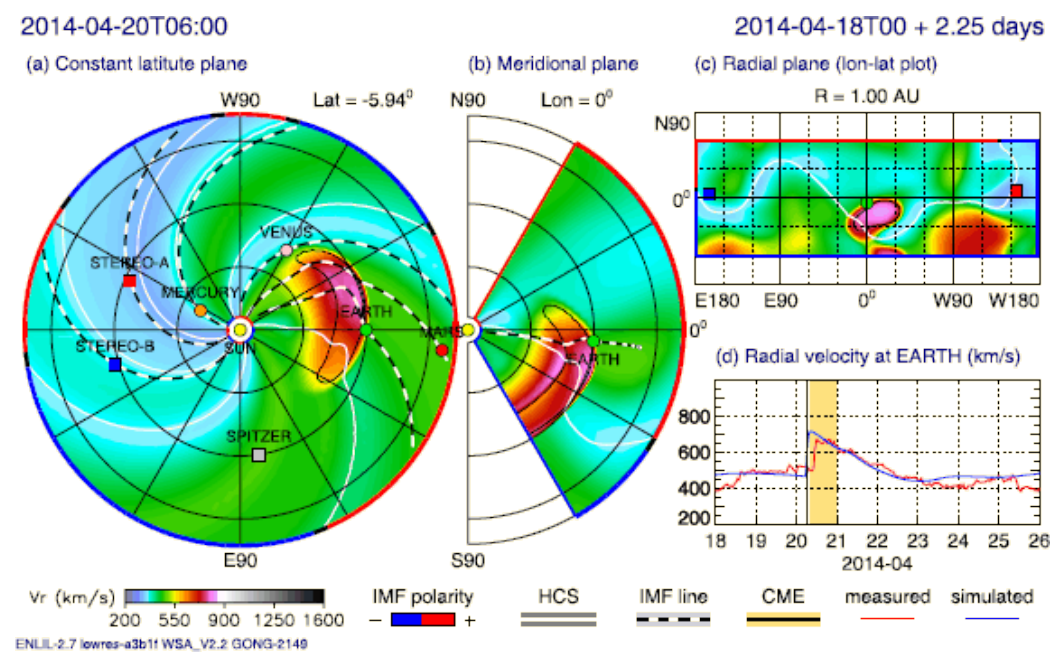

Fig. 10.4: WSA-ENLIL plus Cone ensemble simulation of the April, 182014 CME event with scaled velocity contour plot for the (a) constant latitudinal Earth plane, (b) meridional Earth plane and (c) $1 \mathrm{AU}$ sphere in cylindrical projection. The median of the ensemble CME input parameters are: speed of $1394 \mathrm{~km} / \mathrm{s}$, longitude $9^{\circ}$, latitude of $35^{\circ}$ latitude, and a half-width of $46^{\circ}$. Further, (d) the measured (red) and simulated (blue) radial velocity profiles at Earth, with the simulated CME duration (yellow) are shown. (Mays et al., 2015) 


\section{Bibliography}

Acheson, D. J. 1979, Instability by magnetic buoyancy, Solar Physics, 62, 23

Alfven, H. 1977, Electric currents in cosmic plasmas, Reviews of Geophysics and Space Physics, 15, 271

Aly, J. J. 1984, On some properties of force-free magnetic fields in infinite regions of space, Astrophys. J., 283, 349

-. 1991, How much energy can be stored in a three-dimensional force-free magnetic field?, Astrophys. J., Lett., 375, L61

Amari, T., Aly, J.-J., Luciani, J.-F., Mikic, Z., \& Linker, J. 2011, Coronal Mass Ejection Initiation by Converging Photospheric Flows: Toward a Realistic Model, Astrophys. J., Lett., 742, L27

Amari, T., Luciani, J. F., Aly, J. J., Mikic, Z., \& Linker, J. 2003, Coronal Mass Ejection: Initiation, Magnetic Helicity, and Flux Ropes. I. Boundary Motion-driven Evolution, Astrophys. J., 585, 1073

Andrews, M. 2003, A Search for CMEs Associated with Big Flares, Solar Physics, 218, 261

Antiochos, S. K., DeVore, C. R., \& Klimchuk, J. A. 1999, A Model for Solar Coronal Mass Ejections, Astrophys. J., 510, 485

Arber, T. D., Haynes, M., \& Leake, J. E. 2007, Emergence of a Flux Tube through a Partially Ionized Solar Atmosphere, Astrophys. J., 666, 541

Archontis, V., Hood, A. W., Savcheva, A., Golub, L., \& Deluca, E. 2009, On the Structure and Evolution of Complexity in Sigmoids: A Flux Emergence Model, Astrophys. J., 691, 1276

Aschwanden, M., V. Nitta, N., Wuelser, J.-P., R. Lemen, J., Sandman, A., Vourlidas, A., \& Colaninno, R. 2009, First Measurements of the Mass of Coronal Mass Ejections from the EUV Dimming Observed with STEREO EUVI A+B Spacecraft, 706, 376

Aschwanden, M. J. 2001, An Evaluation of Coronal Heating Models for Active Regions Based on Yohkoh, SOHO, and TRACE Observations, The Astrophysical Journal, 560, 1035

Aschwanden, M. J. 2004, Physics of the Solar Corona. An Introduction (Praxis Publishing Ltd)

Aulanier, G., Démoulin, P., Schrijver, C. J., Janvier, M., Pariat, E., \& Schmieder, B. 2013, The standard flare model in three dimensions. II. Upper limit on solar flare energy, Astron. Astrophys., 549, A66 
Aulanier, G., Janvier, M., \& Schmieder, B. 2012, The standard flare model in three dimensions. I. Strong-to-weak shear transition in post-flare loops, Astron. Astrophys., 543, A110

Aulanier, G., Török, T., Démoulin, P., \& DeLuca, E. E. 2010, Formation of Torus-Unstable Flux Ropes and Electric Currents in Erupting Sigmoids, Astrophys. J., 708, 314

Babcock, H. W. 1961, The Topology of the Sun's Magnetic Field and the 22-YEAR Cycle., Astrophys. J., 133, 572

Baker, D., Daly, E., Daglis, I., Kappenman, J. G., \& Panasyuk, M. 2004, Effects of space weather on technology infrastructure, Space Weather, 2

Baker, D. N. 2009, What Does Space Weather Cost Modern Societies?, Space Weather, 7, $\mathrm{n} / \mathrm{a}, \mathrm{s} 02003$

Baker, D. N., Kanekal, S., Blake, J. B., Klecker, B., \& Rostoker, G. 1994, Satellite anomalies linked to electron increase in the magnetosphere, EOS Transactions, 75, 401

Baker, D. N., Li, X., Pulkkinen, A., Ngwira, C. M., Mays, M. L., Galvin, A. B., \& Simunac, K. D. C. 2013, A major solar eruptive event in July 2012: Defining extreme space weather scenarios, Space Weather, 11, 585, 2013SW000963

Banaszkiewicz, M., Axford, W., \& McKenzie, J. 1998, An analytic solar magnetic field model, Astronomy and Astrophysics, 337, 940

Basu, S., \& Antia, H. M. 2008, Helioseismology and solar abundances, Physics Reports, 457,217

Bein, B. M., Temmer, M., Vourlidas, A., Veronig, A. M., \& Utz, D. 2013, The Height Evolution of the "True" Coronal Mass Ejection Mass derived from STEREO COR1 and COR2 Observations, Astrophys. J., 768, 31

Bemporad, A., Raymond, J., Poletto, G., \& Romoli, M. 2007, A comprehensive study of the initiation and early evolution of a coronal mass ejection from ultraviolet and white-light data, The Astrophysical Journal, 655, 576

Bertaux, J.-L., Leblanc, F., Witasse, O., Quemerais, E., Lilensten, J., Stern, S. A., Sandel, B., \& Korablev, O. 2005, Discovery of an aurora on Mars, Nature, 435, 790

Biermann, L. 1951, Kometenschweife und solare Korpuskularstrahlung, Zeitschrift für Astrophysik, 29, 274

Billings, D. E. 1966, A guide to the solar corona

Bolduc, L. 2002, \{GIC\} observations and studies in the Hydro-Québec power system, Journal of Atmospheric and Solar-Terrestrial Physics, 64, 1793, space Weather Effects on Technological Systems

Boteler, D. 2006, The super storms of August/September 1859 and their effects on the telegraph system, Advances in Space Research, 38, 159 , the Great Historical Geomagnetic Storm of 1859: A Modern Look

Boteler, D. H., Pirjola, R. J., \& Nevanlinna, H. 1998, The effects of geomagnetic disturbances on electrical systems at the earth's surface, Advances in Space Research, 22, 17 
Bothmer, V., \& Daglis, I. 2007, Space Weather: Physics and Effects (Springer)

Bothmer, V., \& Daglis, I. A. 2007, Space Weather - Physics and Effects (Praxis Publishing)

Bothmer, V., \& Schwenn, R. 1994, Eruptive prominences as sources of magnetic clouds in the solar wind, Space Science Reviews, 70, 215

—. 1996, Signatures of fast CMEs in interplanetary space, Advances in Space Research, 17

- 1998, The structure and origin of magnetic clouds in the solar wind, Annales Geophysicae, 16, 1

Bougeret, J. L., et al. 2008, S/WAVES: The Radio and Plasma Wave Investigation on the STEREO Mission, Space Science Reviews, 136, 487

Bouvier, A., \& Wadhwa, M. 2010, The age of the Solar System redefined by the oldest $\mathrm{Pb}-\mathrm{Pb}$ age of a meteoritic inclusion, Nature Geoscience, 3, 637

Bowles, K. L. 1958, Observation of Vertical-Incidence Scatter from the Ionosphere at 41 $\mathrm{Mc} / \mathrm{sec}$, Physical Review Letters, 1, 454

Bray, R. J., \& Loughhead, R. E. 1964, Sunspots

Brodrick, D., Tingay, S., \& Wieringa, M. 2005, X-ray magnitude of the 4 November 2003 solar flare inferred from the ionospheric attenuation of the galactic radio background, Journal of Geophysical Research (Space Physics), 110, A09S36

Brueckner, G. E., et al. 1995a, The Large Angle Spectroscopic Coronagraph (LASCO), Solar Physics, 162, 357

—. 1995b, The Large Angle Spectroscopic Coronagraph (LASCO), Solar Physics, 162, 357

Bryant, D. A., Cline, T. L., Desai, U. D., \& McDonald, F. B. 1962, Explorer 12 Observations of Solar Cosmic Rays and Energetic Storm Particles after the Solar Flare of September 28, 1961, J. Geophys. Research, 67, 4983

Burkepile, J. T., Hundhausen, A. J., Stanger, A. L., St. Cyr, O. C., \& Seiden, J. A. 2004, Role of projection effects on solar coronal mass ejection properties: 1. A study of CMEs associated with limb activity, Journal of Geophysical Research (Space Physics), 109, A03103

Burlaga, L., Ness, N., Acuna, M., Richardson, J., Stone, E., \& McDonald, F. 2008, Observations of the heliosheath and solar wind termination shock by Voyager 2, AGU Fall Meeting Abstracts

Burlaga, L. F., Behannon, K. W., \& Klein, L. W. 1987, Compound streams, magnetic clouds, and major geomagnetic storms, J. Geophys. Research, 92, 5725

Byrne, J. P., Maloney, S. A., McAteer, R. T. J., Refojo, J. M., \& Gallagher, P. T. 2010, Propagation of an Earth-directed coronal mass ejection in three dimensions, Nature Communications, 1, 74

Cane, H. V., Stone, R. G., \& Woo, R. 1982, Velocity of the shock generated by a large east limb flare on August 18, 1979, Geophysical Research Letters, 9, 897 
Cargill, P. J. 2004, On the Aerodynamic Drag Force Acting on Interplanetary Coronal Mass Ejections, Solar Physics, 221, 135

Carley, E. P., McAteer, R. T. J., \& Gallagher, P. T. 2012, Coronal Mass Ejection Mass, Energy, and Force Estimates Using STEREO, Astrophys. J., 752, 36

Carmichael, H. 1964, A Process for Flares, NASA Special Publication, 50, 451

Carrington, R. C. 1858, On the Distribution of the Solar Spots in Latitudes since the Beginning of the Year 1854, with a Map, Mon. Not. R. Astron. Soc., 19, 1

-. 1859a, Description of a Singular Appearance seen in the Sun on September 1, 1859, Mon. Not. R. Astron. Soc., 20, 13

- 1859b, Description of a Singular Appearance seen in the Sun on September 1, 1859, Mon. Not. R. Astron. Soc., 20, 13

Chapman, S., \& Ferraro, V. C. A. 1931, A new theory of magnetic storms, Terrestrial Magnetism and Atmospheric Electricity (Journal of Geophysical Research), 36, 77

-. 1932, A new theory of magnetic storms, Terrestrial Magnetism and Atmospheric Electricity (Journal of Geophysical Research), 37, 147

Charbonneau, P. 2010, Dynamo Models of the Solar Cycle, Living Reviews in Solar Physics, 7,3

Chen, J. 1996, Theory of prominence eruption and propagation: Interplanetary consequences, J. Geophys. Research, 101, 27499

Chen, J., Marqué, C., Vourlidas, A., Krall, J., \& Schuck, P. W. 2006, The Flux-Rope Scaling of the Acceleration of Coronal Mass Ejections and Eruptive Prominences, Astrophys. J., 649,452

Chen, J., et al. 2000, Magnetic Geometry and Dynamics of the Fast Coronal Mass Ejection of 1997 September 9, Astrophys. J., 533, 481

Chen, P. F. 2011, Coronal Mass Ejections: Models and Their Observational Basis, Living Reviews in Solar Physics, 8, 1

Cheng, X., Ding, M. D., Zhang, J., Srivastava, A. K., Guo, Y., Chen, P. F., \& Sun, J. Q. 2014, On the Relationship Between a Hot-channel-like Solar Magnetic Flux Rope and its Embedded Prominence, Astrophys. J., Lett., 789, L35

Cherry, N. 2002, Schumann Resonances, a plausible biophysical mechanism for the human health effects of Solar, Natural Hazards: Journal of the International Society for the Prevention and Mitigation of Natural Hazards, 26, 279

Cid, C., Saiz, E., Guerrero, A., Palacios, J., \& Cerrato, Y. 2015, A Carrington-like geomagnetic storm observed in the 21st century, Journal of Space Weather and Space Climate, 5, A16

Cliver, E. W., Feynman, J., \& Garrett, H. B. 1990, An estimate of the maximum speed of the solar wind, 1938-1989, J. Geophys. Research, 95, 17103 
Connelly, J. N., Bizzarro, M., Krot, A. N., Nordlund, A., Wielandt, D., \& Ivanova, M. A. 2012, The Absolute Chronology and Thermal Processing of Solids in the Solar Protoplanetary Disk, Science, 338, 651

Cranmer, S. R., \& Saar, S. H. 2011, Testing a Predictive Theoretical Model for the Mass Loss Rates of Cool Stars, Astrophys. J., 741, 54

Cremades, H., \& Bothmer, V. 2004, On the three-dimensional configuration of coronal mass ejections, Astron. Astrophys., 422, 307

Crifo, F., Picat, J. P., \& Cailloux, M. 1983, Coronal transients - Loop or bubble, Solar Physics, 83, 143

dal Lago, A., Schwenn, R., \& Gonzalez, W. 2002, in COSPAR Meeting, Vol. 34, 34th COSPAR Scientific Assembly, 828

DeForest, C. E., Howard, T. A., \& McComas, D. J. 2014, Inbound Waves in the Solar Corona: A Direct Indicator of Alfvén Surface Location, The Astrophysical Journal, 787, 124

DeForest, C. E., Howard, T. A., Webb, D. F., \& Davies, J. A. 2016, The utility of polarized heliospheric imaging for space weather monitoring. Space Weather, 14, 32, 2015SW001286

Dmitriev, A. V., Chao, J.-K., Suvorova, A. V., Ackerson, K., Ishisaka, K., Kasaba, Y., Kojima, H., \& Matsumoto, H. 2004, Indirect estimation of the solar wind conditions in 29-31 October 2003, Journal of Geophysical Research: Space Physics, 110

Domingo, V., Fleck, B., \& Poland, A. I. 1995, The SOHO Mission: an Overview, Solar Physics, 162, 1

Droege, W., \& Schlickeiser, R. 1986, Particle acceleration in solar flares, Astrophys. J., 305, 909

Eddy, J. A. 1974, A Nineteenth-century Coronal Transient, Astron. Astrophys., 34, 235

Eyles, C. J., et al. 2009, The Heliospheric Imagers Onboard the STEREO Mission, Solar Physics, 254, 387

Fang, F., Manchester, IV, W., Abbett, W. P., \& van der Holst, B. 2012, Dynamic Coupling of Convective Flows and Magnetic Field during Flux Emergence, Astrophys. J., 745, 37

Feulner, G., \& Rahmstorf, S. 2010, On the effect of a new grand minimum of solar activity on the future climate on Earth, Geophysical Research Letters, 37, L05707

Filippov, B., Martsenyuk, O., Srivastava, A. K., \& Uddin, W. 2015, Solar Magnetic Flux Ropes, Journal of Astrophysics and Astronomy, 36, 157

Forbes, T. G., et al. 2006, CME Theory and Models, Space Science Reviews, 123, 251

Gallagher, P. T., Lawrence, G. R., \& Dennis, B. R. 2003, Rapid Acceleration of a Coronal Mass Ejection in the Low Corona and Implications for Propagation, Astrophys. J., Lett., 588, L53

Galvin, A. B., et al. 2008, The Plasma and Suprathermal Ion Composition (PLASTIC) Investigation on the STEREO Observatories, Space Science Reviews, 136, 437 
Gary, G. A. 2001, Plasma Beta above a Solar Active Region: Rethinking the Paradigm, Solar Physics, 203, 71

Gibson, S. E., Fan, Y., Török, T., \& Kliem, B. 2006, The Evolving Sigmoid: Evidence for Magnetic Flux Ropes in the Corona Before, During, and After CMES, Space Science Reviews, 124, 131

Gilbert, H. R., Serex, E. C., Holzer, T. E., MacQueen, R. M., \& McIntosh, P. S. 2001, Narrow Coronal Mass Ejections, Astrophys. J., 550, 1093

Golub, L., et al. 2007, The X-Ray Telescope (XRT) for the Hinode Mission, Solar Physics, 243,63

Gopalswamy, N. 2004, in Astrophysics and Space Science Library, Vol. 317, The Sun and the Heliosphere as an Integrated System, ed. G. Poletto \& S. T. Suess, 201

Gopalswamy, N. 2010, in 20th National Solar Physics Meeting, ed. I. Dorotovic, 108-130

Gopalswamy, N. 2013, in Astronomical Society of India Conference Series, Vol. 10, Astronomical Society of India Conference Series

Gopalswamy, N. 2016, History and development of coronal mass ejections as a key player in solar terrestrial relationship, Geoscience Letters, 3, 8

Gopalswamy, N., \& Kundu, M. R. 1992, Estimation of the mass of a coronal mass ejection from radio observations, Astrophys. J., Lett., 390, L37

Gopalswamy, N., Lara, A., Lepping, R. P., Kaiser, M. L., Berdichevsky, D., \& St. Cyr, O. C. 2000, Interplanetary acceleration of coronal mass ejections, Geophysical Research Letters, 27, 145

Gopalswamy, N., Lara, A., Yashiro, S., Kaiser, M. L., \& Howard, R. A. 2001, Predicting the 1-AU arrival times of coronal mass ejections, J. Geophys. Research, 106, 29207

Gopalswamy, N., Lara, A., Yashiro, S., Nunes, S., \& Howard, R. A. 2003a, in ESA Special Publication, Vol. 535, Solar Variability as an Input to the Earth's Environment, ed. A. Wilson, 403-414

Gopalswamy, N., Shimojo, M., Lu, W., Yashiro, S., Shibasaki, K., \& Howard, R. A. 2003b, Prominence Eruptions and Coronal Mass Ejection: A Statistical Study Using Microwave Observations, Astrophys. J., 586, 562

Gopalswamy, N., \& Thompson, B. J. 2000, Early life of coronal mass ejections, Journal of Atmospheric and Solar-Terrestrial Physics, 62, 1457

Gopalswamy, N., Yashiro, S., Liu, Y., Michalek, G., Vourlidas, A., Kaiser, M., \& Howard, R. 2005, Coronal mass ejections and other extreme characteristics of the 2003 OctoberNovember solar eruptions, Journal of Geophysical Research: Space Physics, 110

Gosling, J. T. 1993, The solar flare myth, J. Geophys. Research, 98, 18937

Gosling, J. T., Hildner, E., MacQueen, R. M., Munro, R. H., Poland, A. I., \& Ross, C. L. 1974, Mass ejections from the sun - A view from SKYLAB, J. Geophys. Research, 79, 4581 
-. 1975, Direct observations of a flare related coronal and solar wind disturbance, Solar Physics, 40, 439

Gosling, J. T., \& McComas, D. J. 1987, Field line draping about fast coronal mass ejecta - A source of strong out-of-the-ecliptic interplanetary magnetic fields, Geophysical Research Letters, 14, 355

Gosling, J. T., McComas, D. J., Phillips, J. L., \& Bame, S. J. 1991, Geomagnetic activity associated with earth passage of interplanetary shock disturbances and coronal mass ejections, J. Geophys. Research, 96, 7831

Green, L. M., López fuentes, M. C., Mandrini, C. H., Démoulin, P., Van Driel-Gesztelyi, L., \& Culhane, J. L. 2002, The Magnetic Helicity Budget of a cme-Prolific Active Region, Solar Physics, 208, 43

Hale, G. E. 1908a, On the Probable Existence of a Magnetic Field in Sun-Spots, Astrophys. J., 28,315

-. 1908b, SOLAR VORTICES (Contributions from the Mt. Wilson Solar Observatory, No. 26), Astrophys. J., 28, 100

- 1924, The Law of Sun-Spot Polarity, Proceedings of the National Academy of Science, 10,53

Hale, G. E., Ellerman, F., Nicholson, S. B., \& Joy, A. H. 1919, The Magnetic Polarity of Sun-Spots, Astrophys. J., 49, 153

Hapgood, M. A. 1992, Space physics coordinate transformations - A user guide, Planetary and Space Science, 40, 711

Harrison, R. 1991, Coronal transients and their relation to solar flares, Advances in Space Research, 11, 25

Harrison, R., et al. 2016, in EGU General Assembly Conference Abstracts, Vol. 18, EGU General Assembly Conference Abstracts, EPSC2016-10220

Harrison, R. A. 1986, Solar coronal mass ejections and flares, Astron. Astrophys., 162, 283

Harvey, J. W., et al. 1996, The Global Oscillation Network Group (GONG) Project, Science, 272,1284

Heath, T. L., \& Aristarchus of Samos. 1913, Aristarchus of Samos, the ancient Copernicus; a history of Greek astronomy to Aristarchus, together With Aristarchus's Treatise on the sizes and distances of the sun and moon

Hess, P., \& Zhang, J. 2014, Stereoscopic study of the kinematic evolution of a coronal mass ejection and its driven shock from the Sun to the Earth and the prediction of their arrival times, The Astrophysical Journal, 792, 49

Hetherington, B. 1996, A chronicle of pre-telescopic astronomy

Hirayama, T. 1974, Theoretical Model of Flares and Prominences. I: Evaporating Flare Model, Solar Physics, 34, 323

Hood, A. W., Archontis, V., \& MacTaggart, D. 2012, 3D MHD Flux Emergence Experiments: Idealised Models and Coronal Interactions, Solar Physics, 278, 3 
Howard, R. A., Michels, D. J., Sheeley, Jr., N. R., \& Koomen, M. J. 1982, The observation of a coronal transient directed at earth, Astrophys. J., Lett., 263, L101

Howard, R. A., Sheeley, Jr., N. R., Michels, D. J., \& Koomen, M. J. 1985, Coronal mass ejections - 1979-1981, J. Geophys. Research, 90, 8173

Howard, R. A., et al. 2008a, Sun Earth Connection Coronal and Heliospheric Investigation (SECCHI), Space Science Reviews, 136, 67

-. 2008b, Sun Earth Connection Coronal and Heliospheric Investigation (SECCHI), Space Science Reviews, 136, 67

Howard, T. A., \& Tappin, S. J. 2009, Interplanetary Coronal Mass Ejections Observed in the Heliosphere: 1. Review of Theory, Space Science Reviews, 147, 31

http://en.wikipedia.org/wiki/User:PAR. 2004, illustration of Thomson scattering, http:// en.wikipedia.org/wiki/File:Thomson_scattering_geometry.png, [Online; accessed 09-09-16]

Hudson, H. S., Lemen, J. R., St. Cyr, O. C., Sterling, A. C., \& Webb, D. F. 1998, X-ray coronal changes during Halo CMEs, Geophysical Research Letters, 25, 2481

Hundhausen, A. 1995, The solar wind, Introduction to space physics, 91

Hundhausen, A. 1999, in The many faces of the sun: a summary of the results from NASA's Solar Maximum Mission., ed. K. T. Strong, J. L. R. Saba, B. M. Haisch, \& J. T. Schmelz, 143

Hundhausen, A. J. 1987, in Sixth International Solar Wind Conference, ed. V. J. Pizzo, T. Holzer, \& D. G. Sime, 181

Hundhausen, A. J. 1993, Sizes and locations of coronal mass ejections - SMM observations from 1980 and 1984-1989, J. Geophys. Research, 98, 13

Hundhausen, A. J., Sawyer, C. B., House, L., Illing, R. M. E., \& Wagner, W. J. 1984, Coronal mass ejections observed during the solar maximum mission - Latitude distribution and rate of occurrence, J. Geophys. Research, 89, 2639

Illing, R. M. E., \& Hundhausen, A. J. 1985, Observation of a coronal transient from 1.2 to 6 solar radii, J. Geophys. Research, 90, 275

Isavnin, A. 2016, FRiED: A Novel Three-dimensional Model of Coronal Mass Ejections, Astrophys. J., 833, 267

Jing, J., Yurchyshyn, V. B., Yang, G., Xu, Y., \& Wang, H. 2004, On the Relation between Filament Eruptions, Flares, and Coronal Mass Ejections, Astrophys. J., 614, 1054

Joshi, N. C., Srivastava, A. K., Filippov, B., Kayshap, P., Uddin, W., Chandra, R., Prasad Choudhary, D., \& Dwivedi, B. N. 2014, Confined Partial Filament Eruption and its Reformation within a Stable Magnetic Flux Rope, Astrophys. J., 787, 11

Kahler, S., \& Gopalswamy, N. 2009, in THE 31st ICRC, ŁÓDŹ 2009

Kahler, S. W. 1992, Solar flares and coronal mass ejections, Ann. Rev. Astron. Astrophys., 30, 113 
Kahler, S. W., \& Hundhausen, A. J. 1992, The magnetic topology of solar coronal structures following mass ejections, J. Geophys. Research, 97, 1619

Kaiser, M. L., Kucera, T. A., Davila, J. M., St. Cyr, O. C., Guhathakurta, M., \& Christian, E. 2008, The STEREO Mission: An Introduction, Space Science Reviews, 136, 5

Klecker, B., et al. 2006, Energetic Particle Observations, Space Science Reviews, 123, 217

Klein, L. W., \& Burlaga, L. F. 1982, Interplanetary magnetic clouds at 1 AU, J. Geophys. Research, 87, 613

Klimchuk, J. A. 2001, Theory of Coronal Mass Ejections, Washington DC American Geophysical Union Geophysical Monograph Series, 125

-. 2006, On Solving the Coronal Heating Problem, Solar Physics, 234, 41

Knipp, D., et al. 2016, The May 1967 great storm and radio disruption event: Extreme space weather and extraordinary responses, Space Weather, 14, 614

Kopp, R., \& Pneuman, G. 1976, Magnetic reconnection in the corona and the loop prominence phenomenon, Solar Physics, 50, 85

Kosugi, T., et al. 2007, The Hinode (Solar-B) Mission: An Overview, Solar Physics, 243, 3

Kuperus, M., \& Raadu, M. A. 1974, The Support of Prominences Formed in Neutral Sheets, Astron. Astrophys., 31, 189

Kusano, K. 2005, Simulation Study of the Formation Mechanism of Sigmoidal Structure in the Solar Corona, Astrophys. J., 631, 1260

Kwon, R.-Y., Zhang, J., \& Vourlidas, A. 2015, Are Halo-like Solar Coronal Mass Ejections Merely a Matter of Geometric Projection Effects?, Astrophys. J., Lett., 799, L29

Lakhina, G. S., \& Tsurutani, B. T. 2016, Geomagnetic storms: historical perspective to modern view, Geoscience Letters, 3, 5

Lara, A., Gopalswamy, N., Xie, H., Mendoza-Torres, E., PéRez-EríQuez, R., \& Michalek, G. 2006, Are halo coronal mass ejections special events?, Journal of Geophysical Research (Space Physics), 111, A06107

Leblanc, Y., Dulk, G. A., \& Bougeret, J.-L. 1998, Tracing the Electron Density from the Corona to 1au, Solar Physics, 183, 165

Lemen, J. R., et al. 2012, The Atmospheric Imaging Assembly (AIA) on the Solar Dynamics Observatory (SDO), Solar Physics, 275, 17

Lepping, R. P., Berdichevsky, D. B., Wu, C.-C., Szabo, A., Narock, T., Mariani, F., Lazarus, A. J., \& Quivers, A. J. 2006, A summary of WIND magnetic clouds for years 1995-2003: model-fitted parameters, associated errors and classifications, Annales Geophysicae, 24, 215

Lindemann, F. 1919, On the solar wind, Phil. Mag, 38, 674

Liu, C., Lee, J., Karlický, M., Prasad Choudhary, D., Deng, N., \& Wang, H. 2009, Successive Solar Flares and Coronal Mass Ejections on 2005 September 13 from NOAA AR 10808, Astrophys. J., 703, 757 
Liu, Y. D., et al. 2012, Interactions between Coronal Mass Ejections Viewed in Coordinated Imaging and in situ Observations, Astrophys. J., Lett., 746, L15

Low, B. C. 1996, Solar Activity and the Corona, Solar Physics, 167, 217

Luhmann, J. G., et al. 2008, STEREO IMPACT Investigation Goals, Measurements, and Data Products Overview, Space Science Reviews, 136, 117

Lyot, B. 1939, The study of the solar corona and prominences without eclipses (George Darwin Lecture, 1939), Mon. Not. R. Astron. Soc., 99, 580

MacQueen, R., Eddy, J., Gosling, J., Hildner, E., Munro, R., Newkirk Jr, G., Poland, A., \& Ross, C. 1974, The outer solar corona as observed from Skylab: Preliminary results, The Astrophysical Journal, 187, L85

MacQueen, R. M., \& Fisher, R. R. 1983, The kinematics of solar inner coronal transients, Solar Physics, 89, 89

MacTaggart, D. 2011, Flux emergence within mature solar active regions, Astron. Astrophys., 531, A108

MacTaggart, D., \& Haynes, A. L. 2014, On magnetic reconnection and flux rope topology in solar flux emergence, Mon. Not. R. Astron. Soc., 438, 1500

MacTaggart, D., \& Hood, A. W. 2009, On the emergence of toroidal flux tubes: general dynamics and comparisons with the cylinder model, Astron. Astrophys., 507, 995

Magara, T. 2006, Dynamic and Topological Features of Photospheric and Coronal Activities Produced by Flux Emergence in the Sun, Astrophys. J., 653, 1499

Magara, T., \& Longcope, D. W. 2003, Injection of Magnetic Energy and Magnetic Helicity into the Solar Atmosphere by an Emerging Magnetic Flux Tube, Astrophys. J., 586, 630

Malara, F., \& Velli, M. 2001, in IAU Symposium, Vol. 203, Recent Insights into the Physics of the Sun and Heliosphere: Highlights from SOHO and Other Space Missions, ed. P. Brekke, B. Fleck, \& J. B. Gurman, 456

Manchester, IV, W., Gombosi, T., DeZeeuw, D., \& Fan, Y. 2004, Eruption of a Buoyantly Emerging Magnetic Flux Rope, Astrophys. J., 610, 588

Manoharan, P. K., van Driel-Gesztelyi, L., Pick, M., \& Demoulin, P. 1996, Evidence for Large-Scale Solar Magnetic Reconnection from Radio and X-Ray Measurements, Astrophys. J., Lett., 468, L73

Mays, M. L., et al. 2015, Ensemble Modeling of CMEs Using the WSA-ENLIL+Cone Model, Solar Physics, 290, 1775

Mays, M. L., et al. 2015, Propagation of the 7 January 2014 CME and Resulting Geomagnetic Non-event, The Astrophysical Journal, 812, 145

McComas, D. J., Bame, S. J., Barker, P., Feldman, W. C., Phillips, J. L., Riley, P., \& Griffee, J. W. 1998, Solar Wind Electron Proton Alpha Monitor (SWEPAM) for the Advanced Composition Explorer, Space Science Reviews, 86, 563

McComas, D. J., et al. 2009, Global Observations of the Interstellar Interaction from the Interstellar Boundary Explorer (IBEX), Science, 326, 959 
—. 2012, The Heliosphere's Interstellar Interaction - No Bow Shock, Science, 336, 1291

Michalek, G., Gopalswamy, N., Yashiro, S., \& Bronarska, K. 2015, Dynamics of CMEs in the LASCO Field of View, Solar Physics, 290, 903

Mikić, Z., \& Linker, J. A. 1996, The large-scale structure of the solar corona and inner heliosphere, AIP Conference Proceedings, 382, 104

Minnaert, M. 1930, On the continuous spectrum of the corona and its polarisation. With 3 figures. (Received July 30, 1930), Zeitschrift fuer Astrophysik, 1, 209

Montmerle, T., Augereau, J.-C., Chaussidon, M., Gounelle, M., Marty, B., \& Morbidelli, A. 2006, From Suns to Life: A Chronological Approach to the History of Life on Earth 3. Solar System Formation and Early Evolution: the First 100 Million Years, Earth Moon and Planets, 98, 39

Moon, Y.-J., Choe, G. S., Wang, H., Park, Y. D., Gopalswamy, N., Yang, G., \& Yashiro, S. 2002a, A Statistical Study of Two Classes of Coronal Mass Ejections, Astrophys. J., 581,694

-. 2002b, A Statistical Study of Two Classes of Coronal Mass Ejections, Astrophys. J., 581,694

Mouschovias, T. C., \& Poland, A. I. 1978, Expansion and broadening of coronal loop transients - A theoretical explanation, Astrophys. J., 220, 675

Munro, R. H., Gosling, J. T., Hildner, E., MacQueen, R. M., Poland, A. I., \& Ross, C. L. 1979, The association of coronal mass ejection transients with other forms of solar activity, Solar Physics, 61, 201

NASA. 2017, Halloween Storms of 2003 Still the Scariest, https://www.nasa.gov/ topics/solarsystem/features/halloween_storms.html, accessed: 2017-08-03

NASA - Marshall Spaceflight Center. 2017, The Solar Interior

Nishida, K., Nishizuka, N., \& Shibata, K. 2013, The Role of a Flux Rope Ejection in a Three-dimensional Magnetohydrodynamic Simulation of a Solar Flare, The Astrophysical Journal Letters, 775, L39

Odstrcil, D. 2003, Modeling 3-D solar wind structure, Advances in Space Research, 32, 497

Odstrcil, D., Riley, P., \& Zhao, X. P. 2004, Numerical simulation of the 12 May 1997 interplanetary CME event, Journal of Geophysical Research (Space Physics), 109, A02116

Okamoto, T. J., et al. 2008, Emergence of a Helical Flux Rope under an Active Region Prominence, Astrophys. J., Lett., 673, L215

-. 2009, Prominence Formation Associated with an Emerging Helical Flux Rope, Astrophys. J., 697, 913

Oliver Beatson. 2009, Solar Life Cycle, [Online; accessed April 27, 2017]

Opher, M., Bibi, F. A., Toth, G., Richardson, J. D., Izmodenov, V. V., \& Gombosi, T. I. 2009, A strong, highly-tilted interstellar magnetic field near the Solar System, Nature, 462,1036 
Parker, E. N. 1955, The Formation of Sunspots from the Solar Toroidal Field., Astrophys. J., 121, 491

-. 1958, Dynamics of the Interplanetary Gas and Magnetic Fields., Astrophys. J., 128, 664

Patsourakos, S., Vourlidas, A., \& Stenborg, G. 2013, Direct Evidence for a Fast Coronal Mass Ejection Driven by the Prior Formation and Subsequent Destabilization of a Magnetic Flux Rope, Astrophys. J., 764, 125

Pesnell, W. D., Thompson, B. J., \& Chamberlin, P. C. 2012, The Solar Dynamics Observatory (SDO), Solar Physics, 275, 3

Pizzo, V. J. 1985, Interplanetary shocks on the large scale - A retrospective on the last decade's theoretical efforts, Washington DC American Geophysical Union Geophysical Monograph Series, 35, 51

Plunkett, S. P., et al. 2000, Simultaneous SOHO and Ground-Based Observations of a Large Eruptive Prominence and Coronal Mass Ejection, Solar Physics, 194, 371

Poland, A. I., Howard, R. A., Koomen, M. J., Michels, D. J., \& Sheeley, Jr., N. R. 1981, Coronal transients near sunspot maximum, Solar Physics, 69, 169

Priest, E. R., Hood, A. W., \& Anzer, U. 1989, A twisted flux-tube model for solar prominences. I - General properties, Astrophys. J., 344, 1010

Pulkkinen, A., Lindahl, S., Viljanen, A., \& Pirjola, R. 2005, Geomagnetic storm of 29-31 October 2003: Geomagnetically induced currents and their relation to problems in the Swedish high-voltage power transmission system, Space Weather, 3, S08C03

Pulkkinen, A., Lindahl, S., Viljanen, A., \& Pirjola, R. 2005, Geomagnetic storm of 29-31 October 2003: Geomagnetically induced currents and their relation to problems in the Swedish high-voltage power transmission system, Space Weather, 3, n/a, s08C03

R. Cranmer, S. 2017, Mass Loss Rates from Coronal Mass Ejections: A Predictive Theoretical Model for Solar-Type Stars, 840

Rao, U. R., McCracken, K. G., \& Bukata, R. P. 1967, Cosmic-ray propagation processes: 2. The energetic storm-particle event, J. Geophys. Research, 72, 4325

Raychaudhuri, P. 2005, in IAU Symposium, Vol. 226, Coronal and Stellar Mass Ejections, ed. K. Dere, J. Wang, \& Y. Yan, 211-212

Reeves, G. D., et al. 1998, The relativistic electron response at geosynchronous orbit during the January 1997 magnetic storm, J. Geophys. Research, 103, 17559

Richard, R. L., El-Alaoui, M., Ashour-Abdalla, M., \& Walker, R. J. 2002, Interplanetary magnetic field control of the entry of solar energetic particles into the magnetosphere, Journal of Geophysical Research (Space Physics), 107, 1184

Ridgway, C., \& Priest, E. R. 1993, Prominence support in helical coronal fields formed by photospheric motions, Solar Physics, 146, 277

Robbrecht, E., Berghmans, D., \& Van der Linden, R. A. M. 2009, Automated LASCO CME Catalog for Solar Cycle 23: Are CMEs Scale Invariant?, Astrophys. J., 691, 1222 
Rouillard, A. P. 2011, Relating white light and in situ observations of coronal mass ejections: A review, Journal of Atmospheric and Solar-Terrestrial Physics, 73, 1201

Rouillard, A. P., et al. 2008, First imaging of corotating interaction regions using the STEREO spacecraft, Geophysical Research Letters, 35, L10110

-. 2009, A solar storm observed from the Sun to Venus using the STEREO, Venus Express, and MESSENGER spacecraft, Journal of Geophysical Research (Space Physics), 114, A07106

Roussev, I. I. 2008, Eruptive events in the solar atmosphere: new insights from theory and 3-D numerical modelling, Contemporary Physics, 49, 237

Russell, C. 2001, Solar wind and interplanetary magnetic field: A tutorial, Space Weather, 73

Rust, D. M., \& Kumar, A. 1996, Evidence for Helically Kinked Magnetic Flux Ropes in Solar Eruptions, Astrophys. J., Lett., 464, L199

Ruzmaikin, A. A. 1981, The solar cycle as a strange attractor, Comments on Astrophysics, 9,85

Sachdeva, N., Subramanian, P., Vourlidas, A., \& Bothmer, V. 2017, CME Dynamics Using STEREO and LASCO Observations: The Relative Importance of Lorentz Forces and Solar Wind Drag, Solar Physics, 292, 118

Scherrer, P. H., et al. 2012, The Helioseismic and Magnetic Imager (HMI) Investigation for the Solar Dynamics Observatory (SDO), Solar Physics, 275, 207

Schmieder, B., Démoulin, P., \& Aulanier, G. 2013, Solar filament eruptions and their physical role in triggering coronal mass ejections, Advances in Space Research, 51, 1967

Schuster, A. 1879, on the polarisation of the Solar Corona, Mon. Not. R. Astron. Soc., 40, 35

Schwenn, R. 1986, Relationship of coronal transients to interplanetary shocks 3D aspects, Space Science Reviews, 44, 139

Schwenn, R. 2006, Space Weather: The Solar Perspective, Living Reviews in Solar Physics, 3,2

Schwenn, R. 2006, Space Weather: The Solar Perspective, Living Reviews in Solar Physics, 3,2

Schwenn, R., dal Lago, A., Huttunen, E., \& Gonzalez, W. D. 2005, The association of coronal mass ejections with their effects near the Earth, Annales Geophysicae, 23, 1033

Sheeley, N. R., Hakala, W. N., \& Wang, Y.-M. 2000a, Detection of coronal mass ejection associated shock waves in the outer corona, J. Geophys. Research, 105, 5081

- 2000b, Detection of coronal mass ejection associated shock waves in the outer corona, J. Geophys. Research, 105, 5081

Sheeley, N. R., Walters, J. H., Wang, Y.-M., \& Howard, R. A. 1999a, Continuous tracking of coronal outflows: Two kinds of coronal mass ejections, J. Geophys. Research, 104, 24739 
- 1999b, Continuous tracking of coronal outflows: Two kinds of coronal mass ejections, J. Geophys. Research, 104, 24739

Sheeley, N. R., et al. 1997, Measurements of Flow Speeds in the Corona Between 2 and 30 $R$, Astrophys. J., 484, 472

Sheeley, Jr., N. R., Howard, R. A., Michels, D. J., Koomen, M. J., Schwenn, R., Muehlhaeuser, K. H., \& Rosenbauer, H. 1985, Coronal mass ejections and interplanetary shocks, J. Geophys. Research, 90, 163

Sheeley, Jr., N. R., Michels, D. J., Howard, R. A., \& Koomen, M. J. 1980, Initial observations with the SOLWIND coronagraph, Astrophys. J., Lett., 237, L99

Sheeley, Jr., N. R., et al. 2008, Heliospheric Images of the Solar Wind at Earth, Astrophys. J., 675,853

Shen, C., Wang, Y., Pan, Z., Zhang, M., Ye, P., \& Wang, S. 2013, Full halo coronal mass ejections: Do we need to correct the projection effect in terms of velocity?, Journal of Geophysical Research (Space Physics), 118, 6858

Shen, F., Shen, C., Zhang, J., Hess, P., Wang, Y., Feng, X., Cheng, H., \& Yang, Y. 2014, Evolution of the 2012 July 12 CME from the Sun to the Earth: Data-Constrained Three-Dimensional MHD Simulations, 119

Shrivastava, P., \& Jaiswal, A. 2009, Relation between coronal mass ejections and solar activity for solar cycle 23

Slocum, F. 1912, The Study of Solar Prominences, Popular Astronomy, 20, 409

Smith, C. W., L'Heureux, J., Ness, N. F., Acuña, M. H., Burlaga, L. F., \& Scheifele, J. 1998, The ACE Magnetic Fields Experiment, Space Science Reviews, 86, 613

Solovev, A. A. 1985, Dynamics of twisted magnetic loops, Astrofizika, 23, 393

Sonett, C. P., Colburn, D. S., Davis, L., Smith, E. J., \& Coleman, P. J. 1964, Evidence for a Collision-Free Magnetohydrodynamic Shock in Interplanetary Space, Physical Review Letters, 13, 153

St. Cyr, C. 2005, The Last Word: The Definition of Halo Coronal Mass Ejections, EOS Transactions, 86, 281

Stenborg, G., Vourlidas, A., \& Howard, R. A. 2008, A Fresh View of the Extreme-Ultraviolet Corona from the Application of a New Image-Processing Technique, Astrophys. J., 674, 1201

Stix, M. 1989, The Sun. an Introduction, 192

Sturrock, P. A. 1966, Model of the High-Energy Phase of Solar Flares, Nature, 211, 695

-. 1991, Maximum energy of semi-infinite magnetic field configurations, Astrophys. J., 380,655

Sviatsky, D. 1923, L'Astronomie dans les Chroniques Russes., L'Astronomie, 37, 418

Temmer, M., \& Nitta, N. V. 2015a, Interplanetary Propagation Behavior of the Fast Coronal Mass Ejection on 23 July 2012, Solar Physics, 290, 919 
-. 2015b, Interplanetary Propagation Behavior of the Fast Coronal Mass Ejection on 23 July 2012, Solar Physics, 290, 919

Temmer, M., Preiss, S., \& Veronig, A. M. 2009, CME Projection Effects Studied with STEREO/COR and SOHO/LASCO, Solar Physics, 256, 183

Temmer, M., Veronig, A. M., Peinhart, V., \& Vršnak, B. 2014, Asymmetry in the CMECME Interaction Process for the Events from 2011 February 14-15, Astrophys. J., 785, 85

Thernisien, A., Vourlidas, A., \& Howard, R. A. 2009, Forward Modeling of Coronal Mass Ejections Using STEREO/SECCHI Data, Solar Physics, 256, 111

-. 2011, CME reconstruction: Pre-STEREO and STEREO era, Journal of Atmospheric and Solar-Terrestrial Physics, 73, 1156

Thernisien, A. F. R., Howard, R. A., \& Vourlidas, A. 2006, Modeling of Flux Rope Coronal Mass Ejections, Astrophys. J., 652, 763

Thompson, M. J., Christensen-Dalsgaard, J., Miesch, M. S., \& Toomre, J. 2003, The Internal Rotation of the Sun, Ann. Rev. Astron. Astrophys., 41, 599

Thompson, W. T. 2006, Coordinate systems for solar image data, Astron. Astrophys., 449, 791

Thomson, A. W. P., Dawson, E. B., \& Reay, S. J. 2011, Quantifying extreme behavior in geomagnetic activity, Space Weather, 9, S10001

Thomson, J. 1903, Conduction of Electricity Through Gases (University Press)

Török, T., \& Kliem, B. 2004, The kink instability of a coronal magnetic loop as a trigger mechanism for solar eruptions, Publications of the Astronomy Department of the Eotvos Lorand University, 14, 165

-. 2005, Confined and Ejective Eruptions of Kink-unstable Flux Ropes, Astrophys. J., Lett., 630, L97

Török, T., et al. 2014, Distribution of Electric Currents in Solar Active Regions, Astrophys. J., Lett., 782, L10

Tousey, R. 1973, in Space Research Conference, Vol. 2, 713-730

Tousey, R. 1977, Apollo Telescope Mount of SKYLAB - an overview, Applied Optics, 16, 825

Tripathi, D., Bothmer, V., \& Cremades, H. 2004, The basic characteristics of EUV posteruptive arcades and their role as tracers of coronal mass ejection source regions, Astron. Astrophys., 422, 337

Tsurutani, B. T., Gonzalez, W. D., Gonzalez, A. L. C., Tang, F., Arballo, J. K., \& Okada, M. 1995, Interplanetary origin of geomagnetic activity in the declining phase of the solar cycle, J. Geophys. Research, 100, 21717

Tsurutani, B. T., Smith, E. J., Gonzalez, W. D., Tang, F., \& Akasofu, S. I. 1988, Origin of interplanetary southward magnetic fields responsible for major magnetic storms near solar maximum (1978-1979), J. Geophys. Research, 93, 8519 
Tucker-Hood, K., et al. 2015, Validation of a priori CME arrival predictions made using real-time heliospheric imager observations, Space Weather, 13, 35, 2014SW001106

Usoskin, I. G. 2017, A history of solar activity over millennia, Living Reviews in Solar Physics, 14, 3

Žic, T., Vršnak, B., \& Temmer, M. 2015, Heliospheric Propagation of Coronal Mass Ejections: Drag-based Model Fitting, Astrophys. J., Suppl. Ser., 218, 32

Žic, T., Vršnak, B., Temmer, M., \& Jacobs, C. 2008, Cylindrical and Spherical Pistons as Drivers of MHD Shocks, Solar Physics, 253, 237

van Ballegooijen, A. A., \& Martens, P. C. H. 1989, Formation and eruption of solar prominences, Astrophys. J., 343, 971

van de Hulst, H. C. 1950, The electron density of the solar corona, Bull. Astron. Inst. Netherlands, 11, 135

van Driel-Gesztelyi, L., \& Green, L. M. 2015, Evolution of Active Regions, Living Reviews in Solar Physics, 12, 1

Venzmer, M. S., \& Bothmer, V. 2017, Solar-wind predictions for the Parker Solar Probe orbit, ArXiv e-prints

Vourlidas, A. 2015, Mission to the Sun-Earth L L Lagrangian Point: An Optimal Platform for Space Weather Research, Space Weather, 13, 197

Vourlidas, A., Davis, C. J., Eyles, C. J., Crothers, S. R., Harrison, R. A., Howard, R. A., Moses, J. D., \& Socker, D. G. 2007, First Direct Observation of the Interaction between a Comet and a Coronal Mass Ejection Leading to a Complete Plasma Tail Disconnection, Astrophys. J., Lett., 668, L79

Vourlidas, A., \& Howard, R. A. 2006, The Proper Treatment of Coronal Mass Ejection Brightness: A New Methodology and Implications for Observations, Astrophys. J., 642, 1216

Vourlidas, A., Howard, R. A., Esfandiari, E., Patsourakos, S., Yashiro, S., \& Michalek, G. 2010, Comprehensive Analysis of Coronal Mass Ejection Mass and Energy Properties Over a Full Solar Cycle, Astrophys. J., 722, 1522

Vourlidas, A., Lynch, B. J., Howard, R. A., \& Li, Y. 2013, How Many CMEs Have Flux Ropes? Deciphering the Signatures of Shocks, Flux Ropes, and Prominences in Coronagraph Observations of CMEs, Solar Physics, 284, 179

Vourlidas, A., \& Ontiveros, V. 2009, in American Institute of Physics Conference Series, Vol. 1183, American Institute of Physics Conference Series, ed. X. Ao \& G. Z. R. Burrows, 139-146

Vourlidas, A., Subramanian, P., Dere, K. P., \& Howard, R. A. 2000, Large-Angle Spectrometric Coronagraph Measurements of the Energetics of Coronal Mass Ejections, Astrophys. J., 534, 456

Vourlidas, A., Wu, S. T., Wang, A. H., Subramanian, P., \& Howard, R. A. 2003, Direct Detection of a Coronal Mass Ejection-Associated Shock in Large Angle and Spectrometric Coronagraph Experiment White-Light Images, Astrophys. J., 598, 1392 
Vršnak, B., \& Cliver, E. W. 2008, Origin of Coronal Shock Waves. Invited Review, Solar Physics, 253, 215

Vršnak, B., Sudar, D., Ruždjak, D., \& Žic, T. 2007, Projection effects in coronal mass ejections, Astron. Astrophys., 469, 339

Vršnak, B., \& Žic, T. 2007, Transit times of interplanetary coronal mass ejections and the solar wind speed, Astron. Astrophys., 472, 937

Vršnak, B., Žic, T., Falkenberg, T. V., Möstl, C., Vennerstrom, S., \& Vrbanec, D. 2010, The role of aerodynamic drag in propagation of interplanetary coronal mass ejections, Astron. Astrophys., 512, A43

Vršnak, B., et al. 2013, Propagation of Interplanetary Coronal Mass Ejections: The DragBased Model, Solar Physics

Wang, H., Chae, J., Yurchyshyn, V., Yang, G., Steinegger, M., \& Goode, P. R. 2001, InterActive Region Connection of Sympathetic Flaring on 2000 February 17, AGU Spring Meeting Abstracts

Wang, Y.-M. 1998, in Astronomical Society of the Pacific Conference Series, Vol. 154, Cool Stars, Stellar Systems, and the Sun, ed. R. A. Donahue \& J. A. Bookbinder, 131

Wang, Y.-M., \& Stenborg, G. 2010, Spinning Motions in Coronal Cavities, Astrophys. J., Lett., 719, L181

Webb, D. F., \& Howard, R. A. 1994, The solar cycle variation of coronal mass ejections and the solar wind mass flux, J. Geophys. Research, 99, 4201

Webb, D. F., \& Howard, T. A. 2012, Coronal Mass Ejections: Observations, Living Reviews in Solar Physics, 9, 3

Webb, D. F., \& Hundhausen, A. J. 1987, Activity associated with the solar origin of coronal mass ejections, Solar Physics, 108, 383

Wenzel, K.-P., \& Smith, E. J. 1992, The Ulysses Mission: In-ecliptic phase, Geophysical Research Letters, 19, 1235

Wheatland, M. S., \& Craig, I. J. D. 2006, Including Flare Sympathy in a Model for Solar Flare Statistics, Solar Physics, 238, 73

Wild, J. P., Smerd, S. F., \& Weiss, A. A. 1963, Solar Bursts, Ann. Rev. Astron. Astrophys., 1,291

Williams, D. R., Török, T., Démoulin, P., van Driel-Gesztelyi, L., \& Kliem, B. 2005, Eruption of a Kink-unstable Filament in NOAA Active Region 10696, Astrophys. J., Lett., 628, L163

Wilson, R. M. 1987, Geomagnetic response to magnetic clouds, Planetary and Space Science, 35,329

Woskoboinikow, P., Cohn, D., \& Temkin, R. 1983, Application of advanced millimeter/farinfrared sources to collective Thomson scattering plasma diagnostics, International Journal of Infrared and Millimeter Waves, 4, 205 
Yan, Y., Deng, Y., Karlický, M., Fu, Q., Wang, S., \& Liu, Y. 2001, The Magnetic Rope Structure and Associated Energetic Processes in the 2000 July 14 Solar Flare, Astrophys. J., Lett., 551, L115

Yashiro, S., Gopalswamy, N., Michalek, G., \& Howard, R. A. 2003, Properties of narrow coronal mass ejections observed with LASCO, Advances in Space Research, 32, 2631

Yashiro, S., Gopalswamy, N., Michalek, G., St. Cyr, O. C., Plunkett, S. P., Rich, N. B., \& Howard, R. A. 2004, A catalog of white light coronal mass ejections observed by the SOHO spacecraft, Journal of Geophysical Research (Space Physics), 109, A07105

Zhukov, A. N., \& Veselovsky, I. S. 2007, Global Coronal Mass Ejections, Astrophys. J., Lett., 664, L131

Zurbuchen, T. H., \& Richardson, I. G. 2006, In-Situ Solar Wind and Magnetic Field Signatures of Interplanetary Coronal Mass Ejections, Space Science Reviews, 123, 31 


\section{Acknowledgements}

An erster Stelle möchte ich meinem Doktorvater Dr. Volker Bothmer danken für meine Einstellung als Wissenschaftlicher Mitarbeiter und die Möglichkeit bei ihm zu promovieren. Weiterhin danke ich Ihm sehr herzlich für die wissenschaftliche Unterstützung und die stetigig guten Ratschläge. Es hat mich darüber hinaus sehr gefreut jemanden kennenzulernen der gute handgemachte Rockmusik genau so sehr zu schätzen weiß wie ich.

Ein besondere Dank gilt auch meinem Zweitbetreuer Professor Ansgar Reiners, sowie den weiteren Mitgliedern meiner Prüfungskommission.

Für die Unterstützung meines Papers als Co-authoren bedanke ich mich herzlich bei: Niclas Mrotzek, Dr. Angelos Vourlidas, Dr. Volker Bothmer und Dr. Neel Savani.

Weiterhin danken möchte ich bei dem ehemaligen Institutsleiteren Professor Dr. Wolfram Kollatschny für seinen Rückhalt bedanken.

Ich danke allen Institutsmitglieder für die angenehme und freundliche Arbeitsatmosphäre, die ich stets in sehr guter Erinnerung behalten werde.

Ganz wesentlich zu dieser haben auch die stets freundlichen Mitarbeiterinnen unseres Institutssekretariats beigetragen. Deswegen möchte ich mich ganz herzlich bei Klaudia Wolters, Daniela Krone und Nicole Böker bedanken.

Auch möchte ich mich bei Dr. Klaus Reinsch bedanken, dafür dass er meinem Institutsrechner immer schnell und kompetent die Faxen ausgetrieben hat.

Außerdem danke ich den aktuellen und ehemaligen Mitglieder meiner Arbeitsgruppe: Jens Rodmann, Eckhard Bosman, Malte Venzmar, Niclas Mrotzek, Johannes Hinrich und Julius Achenbach.

Dabei gilt mein besondere Dank meinen beiden Bürokollegen und langjährigen Weggefährten Malte Venzmer und Eckhard "Ecki" Bosman. Alle zusammen danken wir George Lukas für Star Wars.

Für übersehene Rechtschreibfehler zeigen sich folgende Personen verantwortlich: F.Reuter, J.Achenbach und J.Hinrichs.

Auch möchte ich mich bei meiner Verbindung, der Wartburg-Coburgia zu Göttingen, bedanken für die vielen lustigen Geschichten und spannenden Abenteuer, die wir zusammen in den letzten Jahren erleben durften.

Zu guter letzt Danke ich meinen Eltern, meinen Großeltern, meiner Schwester und meinem Schwager dafür dass sie mich schon so viele Jahre ausgehalten haben. 


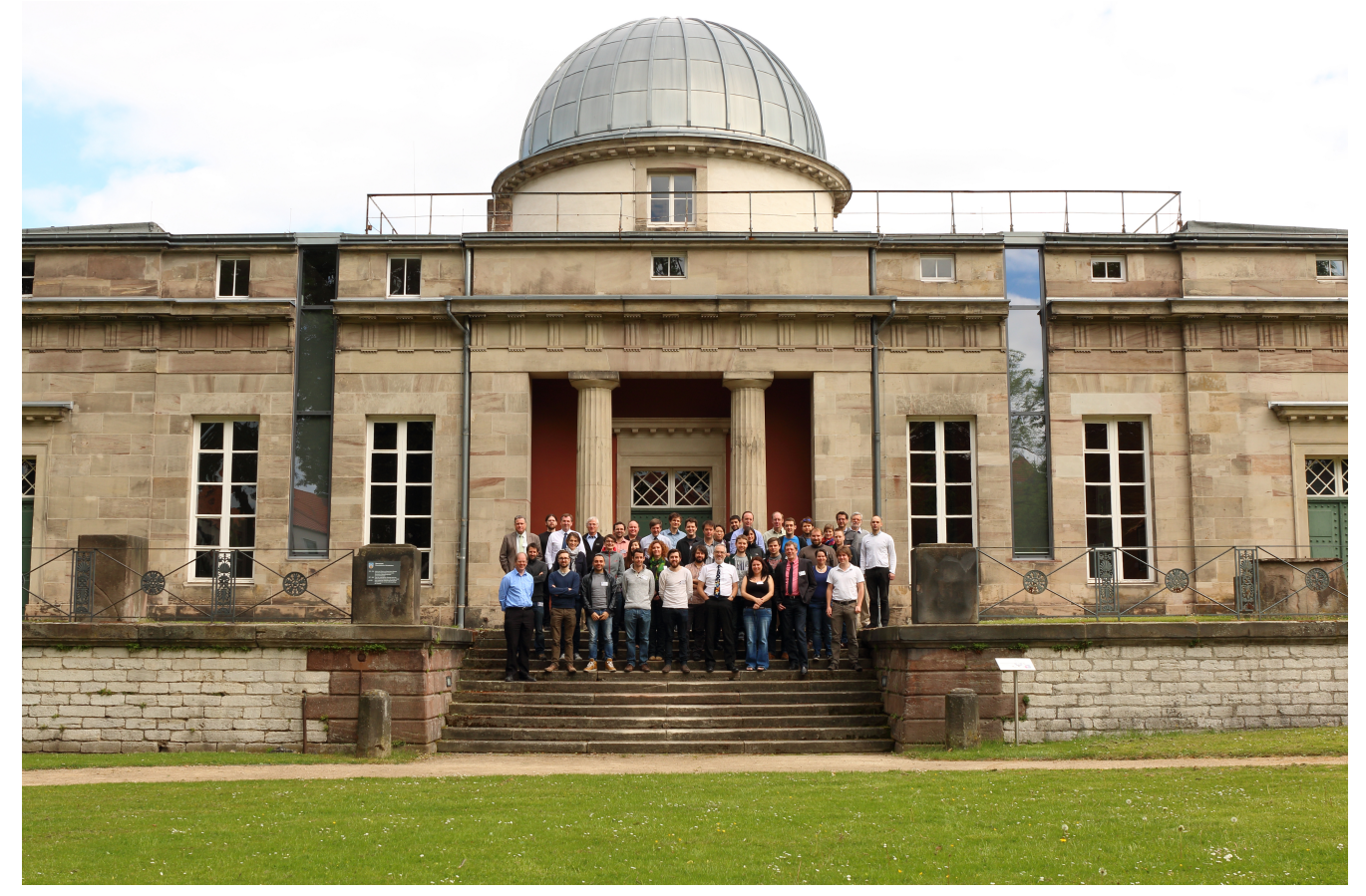

Helcats Meeting Göttingen 2015, Old Observatory

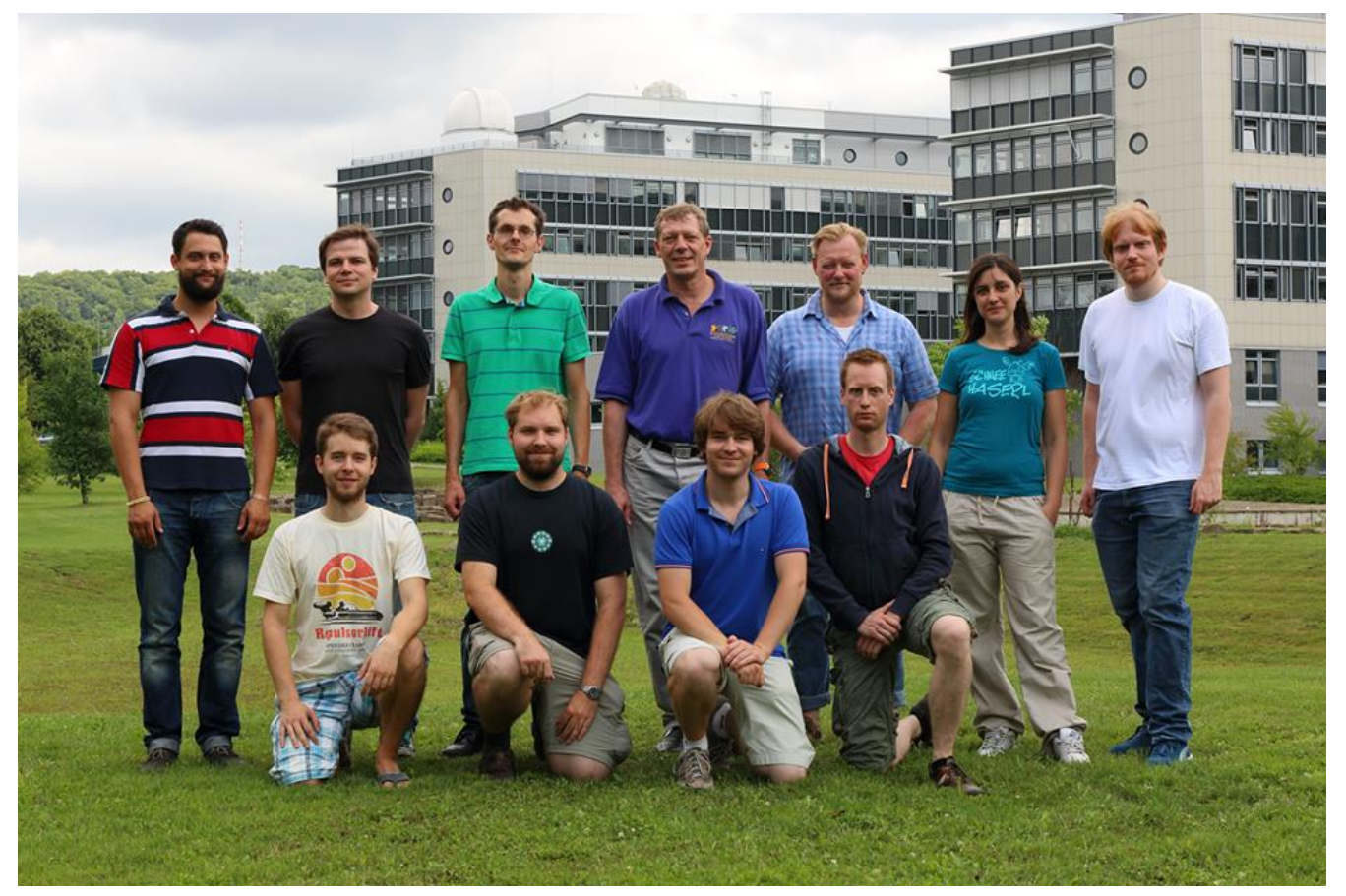

Space Weather Research Group 2015, University of Göttingen 


\section{Declaration}

I hereby declare that the thesis submitted is my own unaided work. All direct or indirect sources used are acknowledged as references. This paper was not previously submitted to another examination board and has not been published.

Göttingen,

(Adam Pluta) 


\title{
Curriculum Vitæ
}

"Es ist schwer, es zugleich der Wahrheit und den Leuten recht zu machen."

- Thomas Mann

\begin{abstract}
Persönliche Daten
Name Adam Pluta

Anschrift Rotenburgerstr. 19, 37269 Eschwege

Telefon +4915737603994

E-Mail adam.pluta@gmx.de

Geburtsdaten 11. November 1980 in Oppeln

Staatsbürgerschaft Deutsch

Familienstand ledig

Zivildienst abgeleistet

Studium

10/2001 - 08/2008 Dipl.-Physiker, Georg-August-Universität, Göttingen, Abschluss. Abschlussprüfung 01/2009, Note: sehr gut

$09 / 2013-$ now

Promotions Student, Georg-August-Universität, Göttingen.

\section{Berufserfahrung}

09/2013-now Wissenschaftlicher Mitarbeiter \& Gleichstellungsbeautragter, Institute for Astrophysics, Göttingen.

09/2009-09/2012 Kernphysiker/Trainee, EnBW \& EDF, Philippsburg/Deutschland \& Cattenom/Frankreich.

10/2006 - 12/2007 Hilfswissenschaftlicher Mitarbeiter, Deutsches Zentrum für Luft \& Raumfahrt, Göttingen.
\end{abstract}

